\title{
Lipid metabolism in
}

Nannochloropsis gaditana during nitrogen starvation

Jorijn H. Janssen 


\section{Propositions}

1. De novo synthesis is important for eicosapentaenoic acid (EPA) accumulation in triacylglycerol (TAG) during nitrogen starvation.

(this thesis)

2. Reporting light intensity without providing reactor dimensions and biomass concentration is useless.

(this thesis)

3. Breakthroughs should not be measured in numbers.

4. Vegetarians who start eating insects decrease sustainability.

5. Crime series give the wrong impression about the complexity of research.

6. Identical twins are not identical.

Propositions belonging to the thesis entitled:

'Lipid metabolism in Nannochloropsis gaditana during nitrogen starvation'

Jorijn H. Janssen

Wageningen, 20 June 2018 


\section{Lipid metabolism in \\ Nannochloropsis gaditana during nitrogen starvation}

Jorijn H. Janssen 


\section{Thesis committee}

\section{Promotor}

Prof. Dr R.H. Wijffels

Professor of Bioprocess Engineering

Wageningen University \& Research

\section{Co-promotor}

Dr M.J. Barbosa

Associate professor, Bioprocess Engineering Group

Wageningen University \& Research

\section{Other members}

Prof. Dr V. Fogliano, Wageningen University \& Research

Prof. M. S. Park, Institute of Hydrobiology, Chinese Academy of Sciences, Hubei Province, China

Dr L. Fourage, Total, Paris, France

Dr I.M. Van der Meer, Wageningen |University \& Research

This research was conducted under the auspices of the Graduate School VLAG (Advanced studies in Food Technology, Agrobiotechnology, Nutrition and Health sciences). 


\title{
Lipid metabolism in
}

\section{Nannochloropsis gaditana}

during nitrogen starvation

\author{
Jorijn $\mathrm{H}$. Janssen
}

\author{
Thesis \\ submitted in fulfilment of the requirements for the degree of doctor \\ at Wageningen University \\ by the authority of the Rector Magnificus, \\ Prof. Dr A.P.J. Mol, \\ in the presence of the
}

Thesis Committee appointed by the Academic Board

to be defended in public

on Friday 20 June 2018

at 4 p.m. in the Aula. 
Jorijn H. Janssen

Lipid metabolism in Nannochloropsis gaditana during nitrogen starvation 164 pages.

PhD thesis, Wageningen University, Wageningen, The Netherlands, 2018 With references, with summaries in English and Dutch

ISBN: 978-94-6343-769-1

DOI: https://doi.org/10.18174/445828 


\section{Contents}

Chapter 1

Introduction

7

Chapter 2 Translocation and de novo synthesis of

eicosapentaenoic acid (EPA) during nitrogen

starvation in Nannochloropsis gaditana

Chapter 3

Expression levels of genes involved in TAG and

polyunsaturated fatty acid synthesis of

Nannochloropsis gaditana during short- and

long-term nitrogen starvation

Chapter 4

Effect of nitrogen addition on lipid productivity of

63

nitrogen starved Nannochloropsis gaditana

Chapter 5 Effect of initial biomass-specific photon supply rate on fatty acid accumulation in nitrogen depleted

Nannochloropsis gaditana under simulated outdoor light conditions

Chapter $6 \quad$ General discussion

References

Summary

Samenvatting

Nomenclature

151

Acknowledgement

154

About the author 


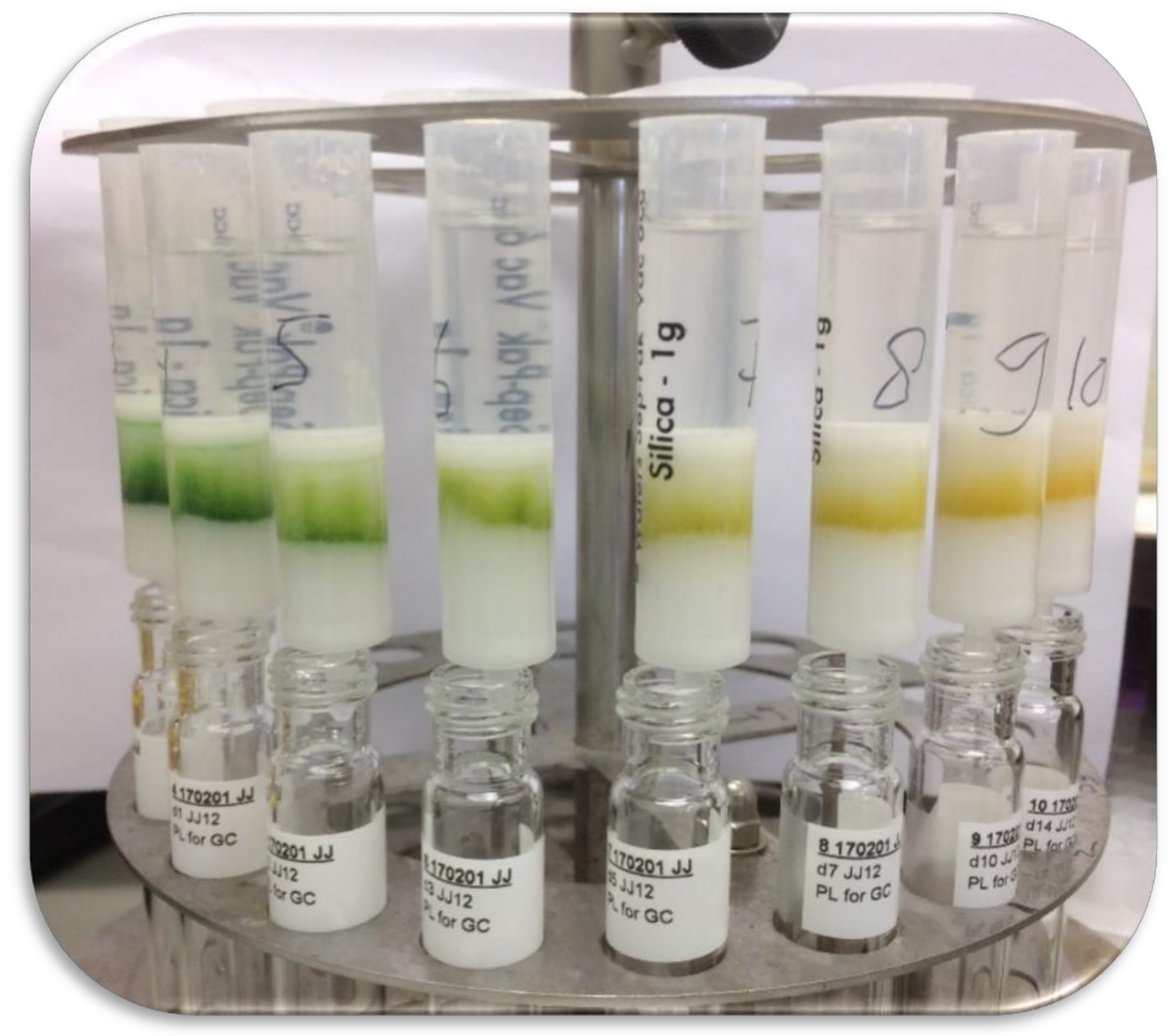

Separation of triacylglycerol (TAG) and polar lipids (PL) using SPE columns. 


\section{Chapter 1}

\section{Introduction and thesis outline}

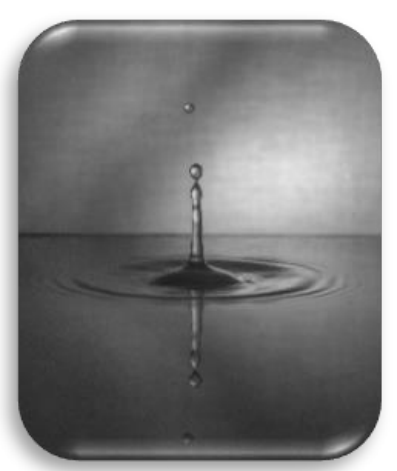


Chapter 1 


\subsection{MICROALGAE AS RENEWABLE LIPID FEEDSTOCK}

The world population and welfare are increasing and therewith the demand of sustainable food, feed and fuel sources are increasing (Godfray et al., 2010). Microalgae can be a sustainable source for these products (Chisti, 2007; Draaisma et al., 2013; Wijffels and Barbosa, 2010). Microalgae are small unicellular photosynthetic organisms which grow using (sun)light, carbon dioxide and nutrients. They produce half of the planets' oxygen, making them essential for life on earth (Chapman, 2013). There are many different algae species, of which only a small fraction is characterized and cultured (Guiry, 2012). The use microalgae has several advantages compared to conventional crops. Microalgae can grow using light and carbon dioxide $\left(\mathrm{CO}_{2}\right)$ and thereby reduce greenhouse gases. They do not compete for freshwater as they can grow in salt water or even use wastewater. Besides, microalgae can be cultivated on non-arable land and thereby not compete with arable land for agriculture. Microalgal areal productivities are much higher compared to conventional crops and can produce a wide variety of products; lipids, carbohydrates, proteins and pigments, depending on the species used. Due to its capacity to produce many different products, microalgae have a wide range of application such as food, aquafeed, biofuels, cosmetics and nutraceuticals. All these advantages make microalgae a potential sustainable feedstock for a transition into a biobased economy. The production costs, however, need to be reduced to achieve an economical feasible process (Ruiz et al., 2016).

\subsection{MICROALGAE NANNOCHLOROPSIS}

The microalga used in this thesis is Nannochloropsis gaditana from the family Eustigmataceae. Nannochloropsis species gained large interest due to their ability to accumulate large amounts of triacylglycerol (TAG) during nitrogen starvation and are natural producers of the omega-3 fatty acid eicosapentaenoic acid (EPA). It has shown high lipid yields compared to other microalgal species (Benvenuti et al., 2015; Radakovits et al., 2012). Nannochloropsis does not accumulate starch and only contain chlorophyll $a$. The cells are small (2-4 $\mu \mathrm{m})$ and round shaped. It can grow under a wide array of culture conditions and has already been cultivated outdoors by several companies (Radakovits et al., 2012). 
There are seven known species of Nannochloropsis: N. gaditana, N. granulata, N. limnetica, N. oceanica, N. oculata, N. salina and N. australis (Fawley et al., 2015). All these are marine species, except for the freshwater species $N$. limnetica. In genetic comparison studies $N$. gaditana and $N$. salina showed so much similarities that they are suggested to be the same species (Starkenburg et al., 2014). In another phylogenetic study, however, N. gaditana and N. salina are suggested to be reclassified to Microchloropsis gaditana and Microchloropsis salina (Fawley et al., 2015). The genome sequences are available for several of these Nannochloropsis species (Corteggiani Carpinelli et al., 2014; Radakovits et al., 2012; Vieler et al., 2012; Wang et al., 2014).

Nannochloropsis gaditana used in this thesis was for the first time described by Lubián, 1982 and isolated from Cádiz bay in Spain. This is also the origin of its name gaditana meaning from Cádiz. The genome sequences for two strains are available; N. gaditana CCMP526 (Radakovits et al., 2012) and N. gaditana B31 (Corteggiani Carpinelli et al., 2014) which enables transcriptome analysis. In addition to the available genome sequences, also genetic transformation methods are available (Kilian et al., 2011; Radakovits et al., 2012).

\subsection{MICROALGAE AS LIPID SOURCE}

This thesis focuses on microalgae as a lipid source. Microalgae have the ability to accumulate up to $60 \%$ lipids of their dry weight (Hu et al., 2008). They accumulate large amounts of triacylglycerol (TAG) in lipid bodies when grown under adverse growth conditions such as nitrogen starvation and high light intensities. The most commonly applied strategy to induce TAG accumulation is nitrogen starvation using a two-phase batch process starting with a growth phase followed by nitrogen starvation. TAG consist of a glycerol backbone with three esterified fatty acids attached (Figure 1.1A). These fatty acids can be saturated and unsaturated fatty acids. Microalgae often accumulate large amounts of the saturated and mono-unsaturated fatty acid palmitic acid and palmitoleic acid but some species can also produce the polyunsaturated omega-3 fatty acid eicosapentaenoic acid (EPA) and accumulate it in both polar lipids and TAG (Figure 1.1B). 


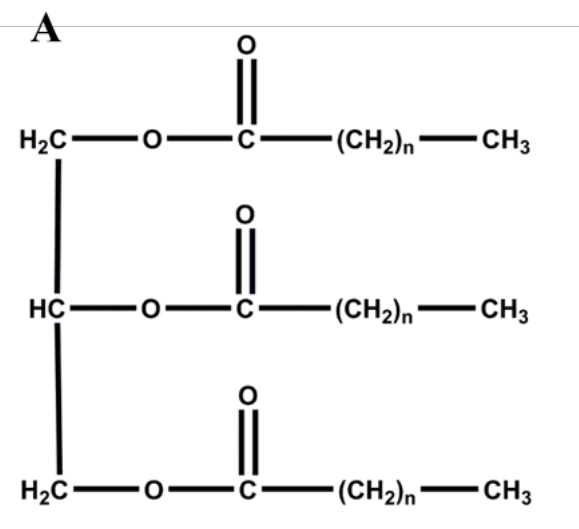

\section{B}<smiles>CCC=CC=CC=CC=CC=CCCCCCCCC(=O)O</smiles>

Figure 1.1. Schematic representation of triacylglycerol (TAG), a glycerol backbone with three fatty acids $(A)$ and the omega-3 fatty acid eicosapentaenoic acid (EPA) (B).

TAG accumulation is hypothesized to be used as sink for excess of electrons and carbon to prevent photo-oxidative stress. TAG production can prevent damage of the cells by regenerating NADPH to NADP+ and can serve as a storage of chloroplast components to be readily available upon more favourable growth conditions. During growth conditions EPA is mainly present in the membranes. The EPA accumulation in TAG in Nannochloropsis increases upon nitrogen starvation (Tonon et al., 2002, Chapters 2 and 3). EPA accumulation in TAG is hypothesized to serve as a storage for membrane lipids for quick use upon more favourable growth conditions. For Nannochloropsis salina the amount of unsaturated fatty acids was inversely correlated with temperature showing its role in membrane fluidity (Van Wagenen et al., 2012). In addition, the double bond in the polyunsaturated fatty acids (PUFAs), like EPA, can scavenge reactive oxygen species (ROS) (Mühlroth et al., 2013).

\subsection{CULTIVATION SYSTEM}

There are many different type of cultivation systems ranging from Erlenmeyer flasks to outdoor photobioreactors. In this research a lab-scale airlift-loop flat panel photobioreactor was used (Figure 1.2) where growth conditions such as temperature, $\mathrm{pH}$ and light intensity are controlled. 


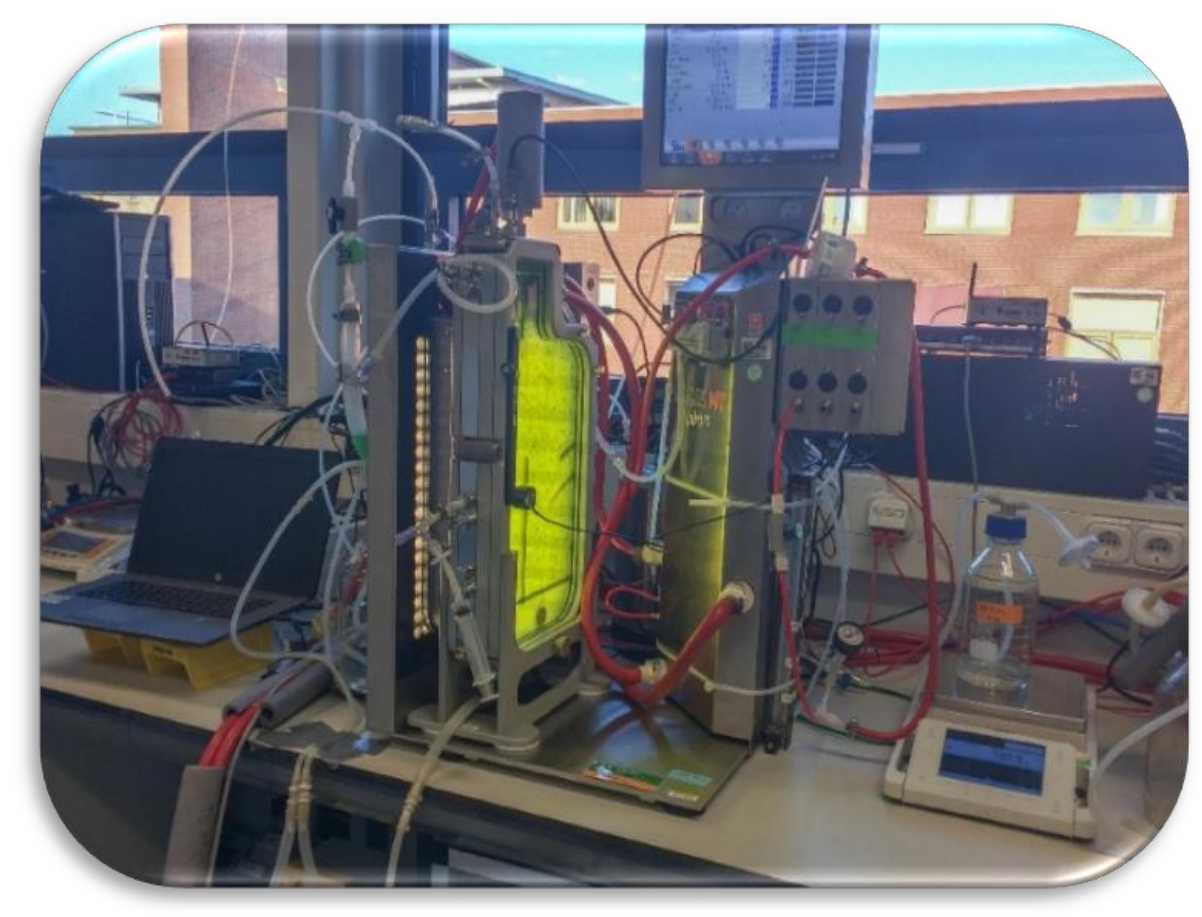

Figure 1.2. Flat-panel photobioreactor setup used in this thesis.

\subsection{AIM AND THESIS OUTLINE}

This thesis aims to increase understanding on the cellular processes underlying lipid accumulation and therewith improve lipid productivity in Nannochloropsis gaditana during nitrogen starvation. Lipid production was studied in this species, mainly focussed on understanding fatty acid accumulation in triacylglycerol (TAG) and polar membrane lipids during nitrogen starvation. Studies were made to elucidate fatty acid translocation between TAG and membrane lipids, de novo fatty acid synthesis and on the gene expression levels related to these metabolic processes at different time points during nitrogen starvation. Due to the commercial potential, focus was put on the polyunsaturated fatty acid EPA. In addition, process strategies to improve TAG productivity were developed and tested.

Several factors, such as light intensity and biomass concentration can influence lipid productivity during nitrogen starvation. Figure $\mathbf{1 . 3}$ shows a schematic overview of the biomass concentrations, light intensities and light regimes used in the different chapters of this thesis. Different starting biomass concentrations and light intensities result in different biomass-specific photon supply rates. 
Biomass concentration

Light intensity
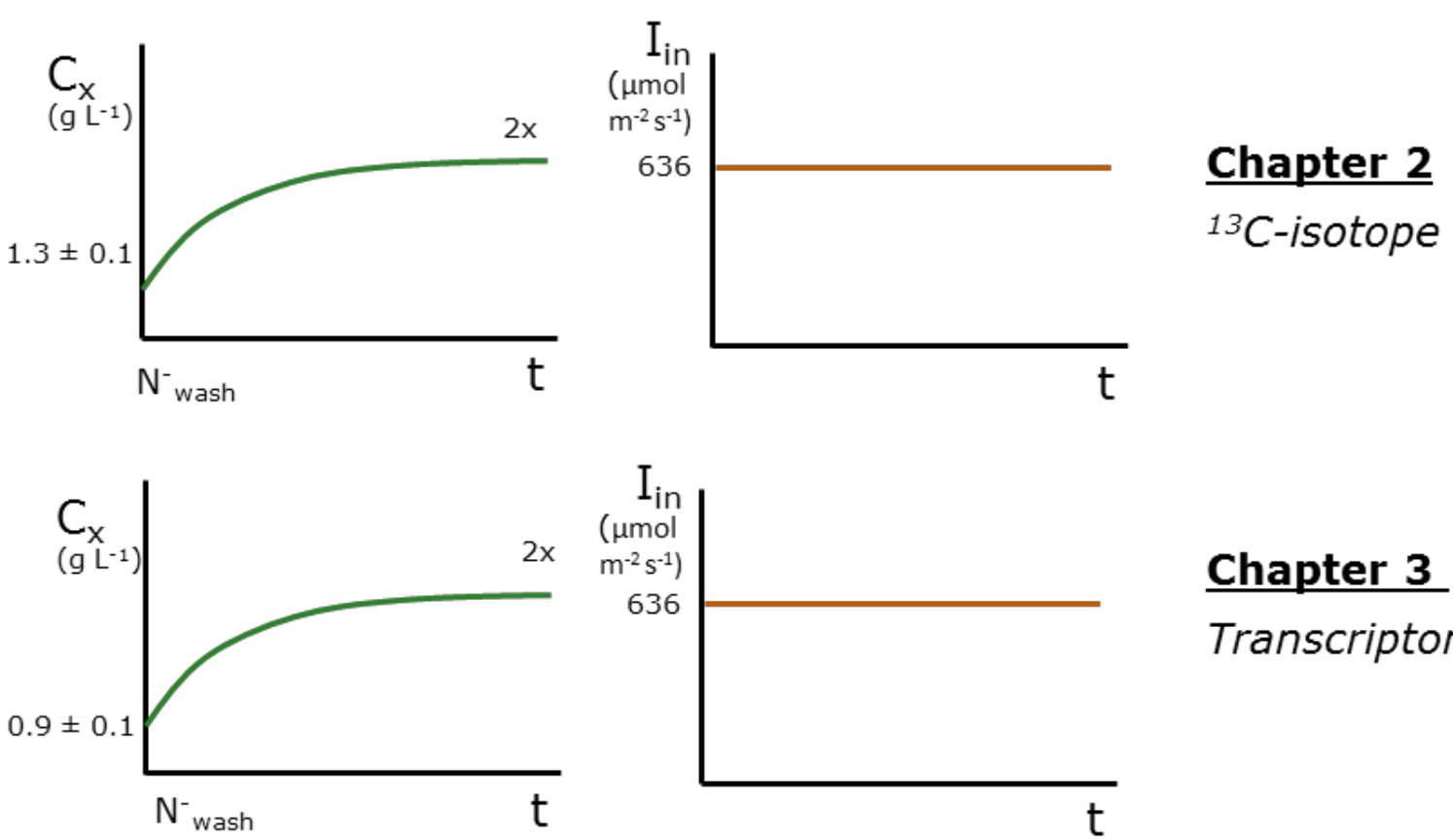

\section{Chapter 3}

Transcriptome
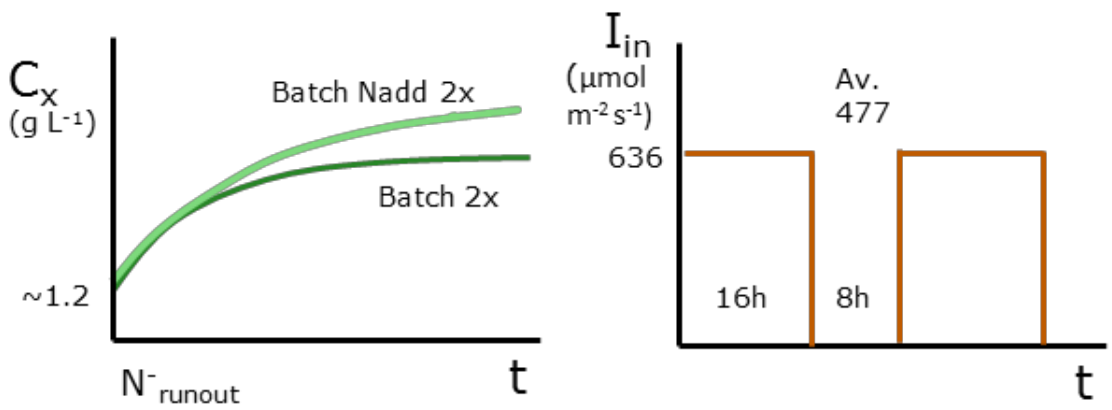

\section{Chapter 4}

Nightly nitrogen addition

\section{Chapter $\mathbf{5}$}

Biomass-specific photon supply rate

Figure 1.3. Overview experimental conditions of the chapters in this thesis. The first column of graphs represent the biomass concentration (Cx) and second column of graphs represent the incident light intensity (lin) and the regime supplied. 
Fatty acids can be present in different fractions within the cell; the polar lipids and TAG. The polar lipids mainly consist of membrane lipids. Upon nitrogen starvation, Nannochloropsis gaditana produces large amount of TAG. The EPA accumulation in TAG increases during nitrogen starvation. It was hypothesized that membrane lipids might be translocated into TAG during nitrogen starvation. The origin of the EPA accumulated in TAG was, however, never determined. Therefore, the fatty acids accumulation in TAG and polar lipids during nitrogen starvation was followed using ${ }^{13} \mathrm{C}$ carbon in Chapter 2. During the growth phase, ${ }^{13} \mathrm{C}$ was used as sole carbon source and at the start of nitrogen starvation, the carbon source was changed to ${ }^{12} \mathrm{C}$. The labelled carbon enables to distinguish newly produced fatty acids from translocated fatty acids.

Little is known about the mechnism of translocation of fatty acids from between lipid fraction in Chapter 3, therefore the transcriptome of Nannochloropsis gaditana was measured during nitrogen starvation. Similar experimental conditions were used as in the research using ${ }^{13} \mathrm{C}$-isotope in Chapter $\mathbf{2}$ in order to compare experiments and associated carbon distribution in the cell (Chapter 2) with gene expression (Chapter $\mathbf{3}$ ) and get more insight in the metabolic pathways responsible for de novo TAG synthesis and translocation of fatty acids. The transcriptome was analysed in different time points during nitrogen starvation to study the differences in expression levels of the genes involved in these pathways. The translocation of fatty acids between lipid fractions can be done via different pathways. One of the enzymes possibly involved in these pathways is phospholipid: diacylglycerol acyltransferase (PDAT) which transports fatty acids from the phospholipids to diacylglycerol (DAG) to form TAG. Other enzymes which could play a role in the translocation of fatty acid are phospholipases which could free fatty acids and thereby be translocated. Next to the genes involved in TAG production, the genes involved in de novo synthesis of EPA were studied.

The TAG production rate is dependent on photosynthetic activity. Upon nitrogen starvation the photosynthetic activity decreases. Addition of nitrogen has been shown to recover the photosystem after nitrogen starvation and increase its photosynthetic efficiency. It was hypothesized that light energy was not efficiently used during nitrogen recovery. Therefore, a new strategy was used in Chapter 4 to improve TAG productivity during nitrogen starvation. Nannochloropsis gaditana was cultivated in a two-phase batch regime with a growth phased followed by nitrogen starvation using a light regime with day:night cycle. During nitrogen starvation phase, small amount of nitrogen are added during the night to improve the photosystem and thereby the TAG productivity without wasting light. 
The nitrogen starvation is always started at a certain biomass concentration which lead to a biomass-specific photon supply rate at a fixed light intensity. The effect on EPA and TAG accumulation is unknown and therefore the effect of different biomass-specific photon supply rates at the start of nitrogen starvation was studied in Chapter $\mathbf{5}$. The biomassspecific photon supply rate was varied by using different biomass concentration at the start of the nitrogen starvation phase and keeping the incident light equal.

In Chapter 6, the results obtained on the EPA and TAG production of Nannochloropsis gaditana during nitrogen starvation under different biomass-specific photon supply rates and light regimes are compared in order to gain insight in the production. All these results were combined and discussed in the general discussion. The EPA accumulation during nitrogen starvation was evaluated in both the polar membrane lipids and TAG lipid fraction. 


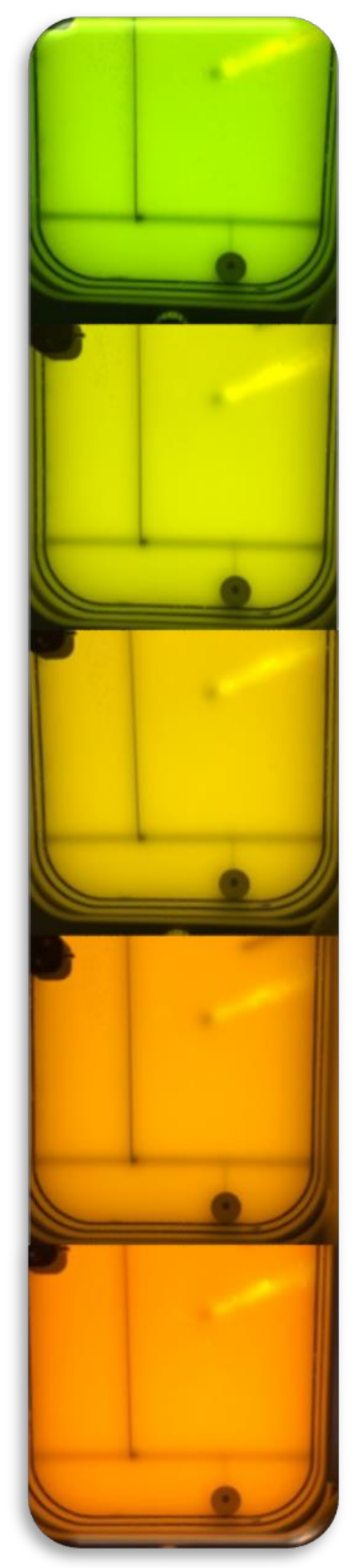

Colour change of the photobioreactor during the first 5 days of nitrogen starvation. 


\section{Chapter 2}

\section{Translocation and de novo}

synthesis of eicosapentaenoic acid

(EPA) during nitrogen starvation in

Nannochloropsis gaditana

This chapter is submitted for publication as:

JH Janssen, PP Lamers, RCH de Vos, RH Wijffels, MJ Barbosa

Translocation and de novo synthesis of eicosapentaenoic acid (EPA) during

nitrogen starvation in Nannochloropsis gaditana 


\section{ABSTRACT}

The microalga Nannochloropsis gaditana is known for accumulating fatty acids, including the commercially interesting eicosapentaenoic acid (EPA) within the polar lipids (PL) and triacylglycerol (TAG) lipids. During microalgal growth EPA is mainly present in the PL. Upon nitrogen starvation N. gaditana accumulates large amounts of TAG in lipid bodies. When expressed per total cell dry weight, the TAG-localized EPA increased while the PL-localized EPA decreased, suggesting that EPA is translocated from the PL into the TAG lipids during nitrogen starvation. Here, we elucidated the origin of EPA in TAG lipid bodies of $N$. gaditana by firstly growing this microalga under optimal growth conditions with ${ }^{13} \mathrm{CO}_{2}$ as the sole carbon source followed by nitrogen starvation with ${ }^{12} \mathrm{CO}_{2}$ as the sole carbon source. By measuring both ${ }^{12} \mathrm{C}$ and ${ }^{13} \mathrm{C}$ fatty acid isotope species in time, the de novo synthesized fatty acids and the already present fatty acids can be distinguished. For the first time, we proved that actual translocation of EPA from the PL into the TAG fraction occurs during nitrogen starvation of $N$. gaditana. Next to being translocated, EPA was synthesized de novo in both PL and TAG during nitrogen starvation. EPA was made by carbon reshuffling within the cell as well. EPA was the main fatty acid translocated, suggesting that the enzyme responsible for fatty acid translocation has a high specificity for EPA.

\section{Graphical abstract}
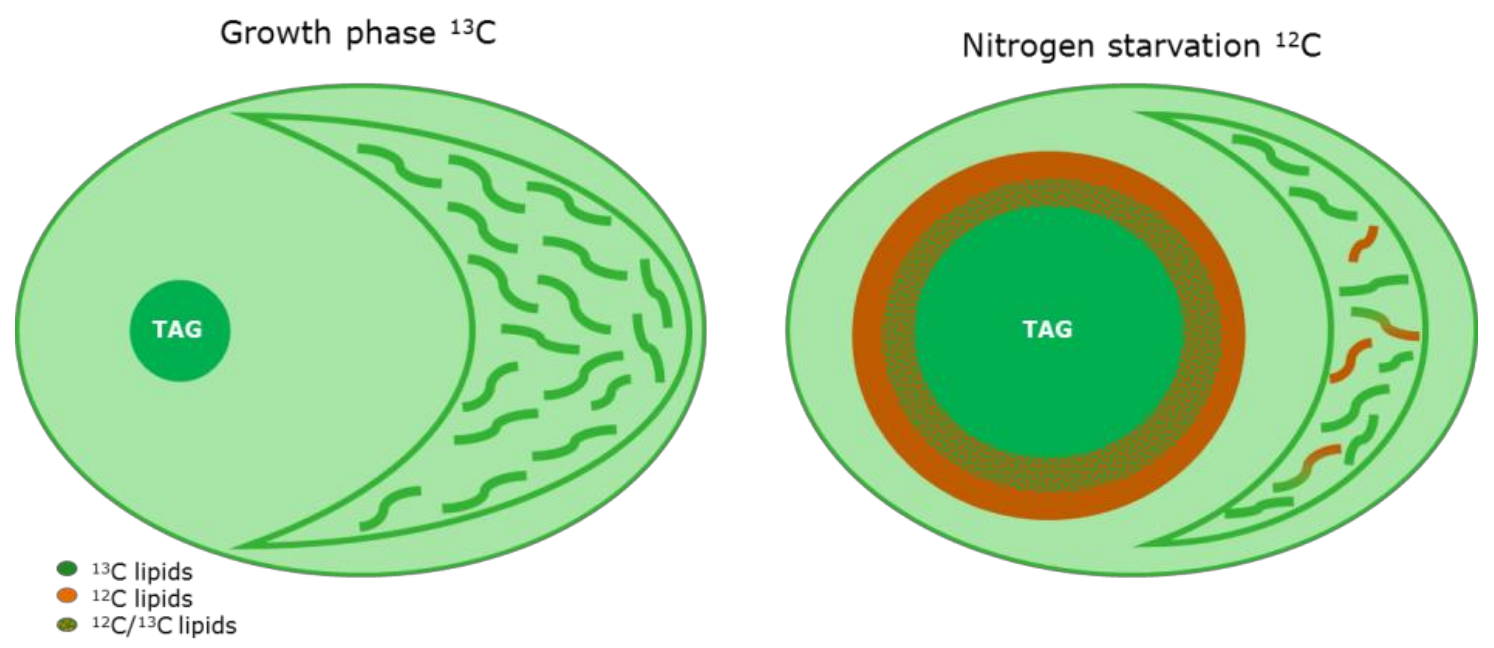


\subsection{INTRODUCTION}

Microalgae naturally produce lipids and can serve as a promising alternative lipid source for the production of food, feed and chemicals (Draaisma et al., 2013; Wijffels and Barbosa, 2010). Nannochloropsis gaditana is a promising marine microalgae species due to its ability to accumulate large amounts of triacylglycerol (TAG) including the high value omega-3 fatty acid eicosapentaenoic acid (EPA) during nitrogen starvation.

In photosynthetic organisms (e.g. microalgae and plants), fatty acids are present in different fractions and location; the polar lipids (PL) are present in membranes and the neutral lipids (triacylglycerol or TAGs) in lipid globules. During growth polyunsaturated fatty acids (PUFAs) like EPA are mainly located in the PL. Nitrogen starvation is known to induce TAG accumulation in lipid bodies in the cytoplasm but also degradation of photosynthetic membranes (Simionato et al., 2013). During nitrogen starvation many fatty acids are synthesized de novo (Hu et al., 2008; Simionato et al., 2013). Next to that, fatty acids can also be translocated between lipid fractions. PUFAs are hypothesized to be translocated from the PL to the TAG fraction, since during nitrogen starvation its content decreases in the PL and increases in TAGs (Hulatt et al., 2017; Remmers et al., 2017b; Simionato et al., 2013). Several functions have been proposed for this TAG accumulation during nitrogen starvation. One of them is to serve as storage of membrane lipid components which later, when cultivation conditions become more favourable, can again be made available for the construction of PUFA-rich chloroplast membranes (Khozin-Goldberg and Cohen, 2011). Using radiolabelling it has been shown that in Parietochloris incisa the PUFA arachidonic acid (C20:4) present in TAGs was used for construction of chloroplast membrane lipids upon nitrogen recovery (Khozin-Goldberg et al., 2005).

The PUFA accumulation into TAG is species and growth phase dependent (Tonon et al., 2002). For different Nannochloropsis species, Phaeodactylum tricornutum, Thalassiosira pseudonana and Pavlova lutheri EPA accumulation into TAG takes place in the stationary phase (Guihéneuf and Stengel, 2013; Hulatt et al., 2017; Remmers et al., 2017b; Tonon et al., 2002). In T. pseudonana and P. lutheri also docosahexaenoic acid (DHA, C22:6) accumulated into TAG (Tonon et al., 2002). In Nannochloropsis the EPA accumulation in TAG correlated with the degradation of EPA-containing membrane glycerolipids (Jia et al., 2015; Simionato et al., 2013). 
In these studies, the overall change in fatty acid accumulation in the cell was measured. Based on the fatty acid mass balances within the different lipid fractions, it was hypothesized that PUFAs were transferred from the PL fraction into TAG upon nitrogen starvation. However, in this way it is not possible to distinguish the proposed translocation of intact free fatty acids between the different lipid fractions from the alternative possibility of de novo fatty acid synthesis in one lipid fraction and simultaneous degradation in another fraction. The actual transfer of intact fatty acids released from (chloroplastic) polar membrane lipids into TAGs upon nitrogen starvation has never been proven. Detailed knowledge on the mechanisms of carbon partitioning during nitrogen starvation is still lacking (Guihéneuf and Stengel, 2013; Mühlroth et al., 2013).

The present study focusses on a better understanding of fatty acid partitioning between TAG and PL during nitrogen starvation, with special interest in EPA, using ${ }^{13} \mathrm{C}$ isotopic labelling of the microalgae Nannochloropsis gaditana. De novo synthesis, degradation and translocation of fatty acids between TAG and PL were elucidated in this work.

\subsection{MATERIALS AND METHODS}

\subsubsection{Microalga, growth medium and pre-cultivation conditions}

The microalgae Nannochloropsis gaditana CCFM-01 (Microalgae Collection of Fitoplancton Marino S.L., CCFM) was used. Pre-cultures were maintained in $250 \mathrm{~mL}$ Erlenmeyer flasks containing $100 \mathrm{~mL}$ culture, in an orbital shaker incubator (100 rpm) with approximately $100 \mu \mathrm{mol} \mathrm{m} \mathrm{m}^{-2}$ continuous light and $2.5 \% \mathrm{CO}_{2}$ enriched air. The growth medium was based on (Breuer et al., 2012) and contained: $\mathrm{NaCl} 445 \mathrm{mM}$; $\mathrm{KNO}_{3} 33.6 \mathrm{mM}$; $\mathrm{Na}_{2} \mathrm{SO}_{4} 3.5 \mathrm{mM} ; \mathrm{MgSO}_{4} \cdot 7 \mathrm{H}_{2} \mathrm{O} 3 \mathrm{mM} ; \mathrm{CaCl}_{2} \cdot 2 \mathrm{H}_{2} \mathrm{O} 2.5 \mathrm{mM} ; \mathrm{K}_{2} \mathrm{HPO}_{4}$ $2.5 \mathrm{mM}$; NaFeEDTA $28 \mu \mathrm{M}$; Na $\mathrm{EDDTA}_{2} \mathrm{H}_{2} \mathrm{O} 80 \mu \mathrm{M}$; $\mathrm{MnCl}_{2} \cdot 4 \mathrm{H} 2 \mathrm{O} 19 \mu \mathrm{M}$; ZnSO${ }_{4} \cdot 7 \mathrm{H}_{2} \mathrm{O}$ $4 \mu \mathrm{M}$; $\mathrm{CoCl}_{2} \cdot 6 \mathrm{H}_{2} \mathrm{O} 1.2 \mu \mathrm{M}$; $\mathrm{CuSO}_{4} \cdot 5 \mathrm{H}_{2} \mathrm{O} 1.3 \mu \mathrm{M}$; $\mathrm{Na}_{2} \mathrm{MoO}_{4} \cdot 2 \mathrm{H}_{2} \mathrm{O} 0.1 \mu \mathrm{M}$; Biotin $0.1 \mu \mathrm{M}$; vitamin B1 $3.3 \mu \mathrm{M}$; and vitamin B12 0.1 $\mu \mathrm{M}$. The $\mathrm{pH}$ was adjusted with $\mathrm{NaOH}$ to $\mathrm{pH}$ 7.5. During pre-cultivation and maintenance of the cultures in Erlenmeyer flasks 100 mM 4-(2-hydroxyethyl)piperazin-1-ethanesulfonic acid (HEPES) was added to the culture medium. In nitrogen free medium $\mathrm{KNO}_{3}$ was replaced by $33.5 \mathrm{mM} \mathrm{KCl}$ to keep equal osmolarity. 
During growth phase $6 \mathrm{~g} \mathrm{NaH}^{13} \mathrm{CO}_{3}$ (Sigma Aldrich) dissolved in $100 \mathrm{~mL}$ growth medium was added to the photobioreactor as carbon source at days 0,3 and 6 . The growth medium was filter sterilized ( $0.22 \mu \mathrm{m}$ filtered, Minisart, Sartorius Stedim) and flushed with nitrogen gas to remove any air left. At the start of the nitrogen starvation phase $0.84 \mathrm{~g} \mathrm{~L}^{-1} \mathrm{NaH}^{12} \mathrm{CO}_{3}$ was added to the nitrogen free medium.

\subsubsection{Experimental approach}

In this research, ${ }^{13} \mathrm{C}$ isotopic labelling was used to study the lipid metabolism in $N$. gaditana and be able to distinguish between de novo synthesis, translocation and carbon reshuffling during nitrogen starvation. Fatty acid accumulation in the PL and TAG fractions in nitrogen starved $N$. gaditana was followed using ${ }^{13} \mathrm{C}$ isotope labelling. First, $N$. gaditana was cultivated with ${ }^{13} \mathrm{C}$-bicarbonate as sole carbon source in a closed photobioreactor to ensure that all carbon in the biomass consists of ${ }^{13} \mathrm{C}$ at the start of the starvation period. Secondly, the nitrogen starvation phase is started and the carbon source is changed to ${ }^{12} \mathrm{CO}_{2}$. The biomass concentration and fatty acid profiles in the TAG and PL fractions are measured over time during nitrogen starvation. The fatty acids were quantified as their fatty acid methyl esters (FAMEs) using gas chromatography coupled to flame ionization detection (GC-FID). Liquid chromatography coupled to high resolution mass spectrometry (LC-MS) was used to enable distinction between ${ }^{13} \mathrm{C}$ and ${ }^{12} \mathrm{C}$ isotopes. The experiment was performed twice as biological duplicate.

\subsubsection{Photobioreactor setup for the growth phase}

The experiment was performed in a batch-operated, heat-sterilized airlift-loop flat panel photobioreactor with a light path of $20.7 \mathrm{~mm}, 1.8 \mathrm{~L}$ working volume and $0.08 \mathrm{~m}^{2}$ surface area (Labfors 5, Infors HT, Switzerland, 2010). During the growth phase the photobioreactor was mixed by recirculation of nitrogen gas in an almost gas tight photobioreactor to prevent ${ }^{12} \mathrm{CO}_{2}$ and oxygen from entering the reactor and the loss of ${ }^{13} \mathrm{C}$ from the reactor (Figure S2.4). The air that could still enter the reactor was estimated to be less than $800 \mathrm{~mL}$ air per day based on the off-gas measurements after gas recirculation with a mass spectrometer (PRIMA $\delta B$ process MS, Thermo Scientific, USA), which was acceptable due to the low $\mathrm{CO}_{2}$ concentration in air. To prevent oxygen inhibition by oxygen accumulation, we increased the gas volume by including a $10 \mathrm{~L}$ gas tank within the gas recirculation setup. During the growth phase the gas was recirculated with a membrane pump, placed inside this gas tank to 
prevent gas exchange with the air, because membrane pumps always tend to draw some ambient air (KNF membrane vacuum pump, type NMS 020B). The nitrogen gas recirculation flow supplied for mixing the culture was approximately $1.0-1.5 \mathrm{~L} \mathrm{~min}^{-1}$. Prior to starting the experiment, the culture medium in the reactor was flushed with nitrogen gas to remove any remaining oxygen and ${ }^{12} \mathrm{CO}_{2}$. Similar growth was observed for cultures in reactors with and without gas recirculation. The $\mathrm{pH}$ was controlled at 7.5 by on-demand addition of $0.9 \mathrm{M}$ sulphuric acid ( $5 \% \mathrm{v}: \mathrm{v})$. The culture temperature was maintained at $26{ }^{\circ} \mathrm{C}$ by water recirculation through the water jacket in contact with the reactor cultivation chamber. The culture was illuminated on the culture side of the reactor by 260 LED light with a warm white spectrum (450-620 nm). During the growth phase the outgoing light intensity was kept at around $30 \mu \mathrm{mol} \mathrm{m} \mathrm{m}^{-2} \mathrm{~s}^{-1}$ by daily increasing the incident light intensity up to $636 \mu \mathrm{mol} \mathrm{m} \mathrm{m}^{2} \mathrm{~s}^{-1}$ on the day before nitrogen starvation was started. The added $\mathrm{NaH}^{13} \mathrm{CO}_{3}$ was used as sole carbon source during the growth phase. The reactor was inoculated at low unlabelled biomass concentration $\left(0.03 \mathrm{~g} \mathrm{~L}^{-1}\right)$ to ensure that more than $98 \%$ of cells consisted of ${ }^{13} \mathrm{C}$ when the nitrogen starvation phase was started.

\subsubsection{Photobioreactor setup for nitrogen starvation phase}

After the growth phase the nitrogen starvation was started by centrifuging the biomass (800 $\mathrm{g}$ for 15 minutes), washing with nitrogen-free growth medium and re-suspending the culture with nitrogen-free growth medium to remove the remaining nitrate and ${ }^{13} \mathrm{C}$. The nitrogen starvation phase was started at a biomass concentration of $1.3 \pm 0.1 \mathrm{~g} \mathrm{~L}^{-1}$ for the two biological duplicates. During the nitrogen starvation phase, a similar reactor setup to the one described above was used, except that the gas was not recirculated and $2 \%{ }^{12} \mathrm{CO}_{2}$ in nitrogen gas was used as carbon source. The incident light intensity was kept continuously at $636 \mu \mathrm{mol} \mathrm{m} \mathrm{m}^{-1}$ during this phase.

\subsubsection{Offline measurements}

Culture growth was assessed by measuring dry weight of the culture in triplicate according to Kliphuis et al. 2012 with the exception that ammonium formate (0.5 M) was used to dilute the sample and wash the filter (Kliphuis et al., 2012). The optical density was measured in duplicate at wavelengths 750, 680 and $480 \mathrm{~nm}$ with UVVIS-spectrophotometer (DR 6000, Hach Lange, Germany, light path $10 \mathrm{~mm}$ ). Cell concentration and volume were measured using the Multisizer III (Beckman Coulter 
Inc., USA) using a $50 \mu \mathrm{m}$ aperture tube. Samples were diluted in ISOTON II diluent. Cell concentration was only measured during one of the duplicate experiments. The average dry weight-specific optical cross section $\left(\alpha_{c}\right.$ in $\left.\mathrm{m}^{2} \mathrm{~g}^{-1}\right)$ was measured and calculated according to de Mooij et al. 2015 using the absorbance from 400 to $750 \mathrm{~nm}$ with a steps size of $1 \mathrm{~nm}$. The absorbance was measured in UV-VIS/doublebeam spectrophotometer (Shimadzu UV-2600, Japan, light path: $2 \mathrm{~mm}$ ) equipped with integrating sphere (ISR-2600) for samples diluted to an optical density $750 \mathrm{~nm}$ of 0.3-0.5 (de Mooij et al., 2015). The dry weight-specific optical cross section was calculated using the measured dry weight concentration $\left(\mathrm{g} \mathrm{L}^{-1}\right)$. The photosystem II (PSII) maximum quantum yield $\left(\mathrm{F}_{\mathrm{v}} / \mathrm{F}_{\mathrm{m}}\right)$ was measured at $455 \mathrm{~nm}$ with AquaPen-C AP-C 100 (Photon Systems Instruments, Czech Republic) after 15 minutes dark adaptation at room temperature.

\subsubsection{Biomass samples}

Biomass samples for fatty acid analysis were taken at day 0, 1, 2, 3, 5, 7, 10 and 14 from the moment of nitrogen starvation. The biomass samples (approximately $60 \mathrm{~mL}$ ) were centrifuged for 5 minutes at $4255 \mathrm{~g}$ (Beckman coulter, Allegra X-30R centrifuge) and washed twice with ammonium formate ( $0.5 \mathrm{M})$. The biomass pellets were stored at $-20^{\circ} \mathrm{C}$ and lyophilized. The measured dry weight was corrected for the dry weight of the reddish supernatant left after centrifugation, as this was washed away before biomass composition analysis.

\subsubsection{Fatty acid analysis}

The fatty acids within the triacylglycerol (TAG) and polar lipids (PL) were quantified with GC-FID according to (Breuer et al., 2012; León-Saiki et al., 2017). In short, lipids were extracted from 6-7 mg lyophilized biomass with chloroform: methanol (1:1.25, v:v) mixture with $237 \mu \mathrm{g} \mathrm{ml} \mathrm{m}^{-1}$ tripentadecanoin (T4257, Sigma Aldrich) and $170 \mathrm{mg} \mathrm{ml}^{-1}$ 1,2-dipentadecanoyl-sn-glycero-3-[phospho-rac-(1-glycerol)] (sodium salt) (840446P, Avanti Polar Lipids Inc.) as internal standard for the TAG and the PL fraction, respectively. The TAG lipids were separated from the PL on SPE silica gel cartridge (Sep-Pak Vac 6cc, Waters). TAG was eluted with a solution of hexane:diethylether $(7: 1, v: v)$ and the $\mathrm{PL}$ were eluted with a solution of methanol:acetone:hexane $(2: 2: 1, \mathrm{v}: \mathrm{v}: \mathrm{v})$. The separated fractions were divided for analysis by GC-FID and LC-MS before organic solvents were evaporated. 
For analysis on the GC-FID the fatty acids were saponified and methylated in methanol with $5 \% \mathrm{H}_{2} \mathrm{SO}_{4}$ at $70{ }^{\circ} \mathrm{C}$ for 3 hours. The fatty acids methyl esters (FAMEs) obtained were extracted with hexane and quantified on the GC-FID (Agilent 7890) (Breuer et al., 2012). The TAG and PL fatty acid contents were calculated by summing all fatty acids measured per mg of biomass in each lipid fraction.

For LC-MS analysis the extracted lipids were saponified without subsequent methylation (Bromke et al., 2015). In short, the separated lipids were suspended in $200 \mu \mathrm{L}$ of methanol with $6 \% \mathrm{KOH}$ in $\mathrm{MQ}(4: 1, \mathrm{v}: \mathrm{v})$, incubated for 2 hours at $60^{\circ} \mathrm{C}$ and cooled to room temperature. $100 \mu \mathrm{L}$ saturated $\mathrm{NaCl}$ was then added and the mixture acidified by adding $50 \mu \mathrm{L}$ of $29 \% \mathrm{HCl}$. The samples were thoroughly mixed and centrifuged for 30 seconds at $20238 \mathrm{~g}$ (Eppendorf centrifuge 5424). The free fatty acids were extracted three times with $200 \mu \mathrm{L}$ chloroform: heptane (1:4, v:v), pooled and washed with $200 \mu \mathrm{L}$ water. The fatty acid fraction was separated and dried by evaporation of the solvent and stored at $-20{ }^{\circ} \mathrm{C}$ until use. Before analysis the dried fatty acids were dissolved in ethanol with $0.1 \%$ butylhydroxytoluene and sonicated for 5 minutes and centrifuged. The samples were analysed by LC-Orbitrap FTMS.

\subsubsection{LC-MS analysis}

Fatty acids present in the saponified lipid fractions were detected by high resolution LC-MS using an Acquity UPLC (Waters, Milford, MA, USA) connected to an LTQOrbitrap XL hybrid mass spectrometer (Thermo Fischer Scientific, San Jose, CA, USA). A Waters Acquity HSS T3 column $(1.8 \mu \mathrm{m}$ particles; $2.1 \times 150 \mathrm{~mm})$ at $55{ }^{\circ} \mathrm{C}$ and a gradient of $20 \%$ acetonitrile in water containing $10 \mathrm{mM}$ ammonium acetate and $0.1 \%$ formic acid (eluent $\mathrm{A}$ ) and $10 \%$ acetonitrile in isopropanol containing $10 \mathrm{mM}$ ammonium acetate and $0.1 \%$ formic acid (eluent $B$ ) were used to separate the compounds. The solvent gradient started with an increase from $35-70 \%$ B in 3 min, then from $70-85 \%$ B in $6 \mathrm{~min}$ and finally from $85-90 \%$ B in $13 \mathrm{~min}$ followed by a washing step at $90 \% \mathrm{~B}$ for another $5 \mathrm{~min}$. The flow rate was $200 \mu \mathrm{l} / \mathrm{min}$. Masses were recorded at a resolution of 60,000 in negative electrospray ionization mode and in the $\mathrm{m} / \mathrm{z}$ range of $105-1400$. Mass calibration and other settings of the MS were as described by Hooft et al. 2012 (Hooft et al., 2012).

Raw LC-MS data were processed using MetAlign software (Lommen and Kools, 2012) for noise estimation, peak picking and alignment of mass features across samples, resulting in a large table (peak list) with the relative intensities, expressed as peak 
heights, of nearly 7,500 mass features in each sample. Mass features corresponding to the molecular ions, $[\mathrm{M}-\mathrm{H}]$; , of both fully ${ }^{12} \mathrm{C}$ and fully ${ }^{13} \mathrm{C}$ isoforms of fatty acids of interest, as well as all possible partially labelled combinations thereof, were identified based on their calculated exact masses, allowing a maximum mass deviation of $3 \mathrm{ppm}$. The relative intensities of these molecular ion peaks were used for further data analysis.

Using the correlation of each sample between the peak intensities of the fatty acids detected by LC-MS and the quantified fatty acids of the GC-FID, the relative LCMS-peak intensities were converted into fatty acid concentrations.

\subsubsection{Calculation time-averaged TAG yield on light}

The time-averaged TAG yield on light $\left(\mathrm{Y}_{\mathrm{TAG} / \mathrm{ph}}\right.$ in $\left.\mathrm{g}_{\mathrm{TAG}} \mathrm{mol}_{\mathrm{ph}}{ }^{-1}\right)$ was calculated as described by Remmers et al., 2017b. The amount of TAG produced over a period was divided by the amount of light supplied in that period. The light necessary for inoculum production was included in the light supplied, and was calculated from the biomass concentration at the start of nitrogen starvation and a theoretical biomass yield on light of $1 \mathrm{~g}_{\mathrm{x}} \mathrm{mol}_{\mathrm{ph}}{ }^{-1}$ (Remmers et al., 2017b).

\subsection{RESULTS AND DISCUSSION}

The fatty acid accumulation in the TAG and PL fraction was studied in Nannochloropsis gaditana during nitrogen starvation. A distinction between de novo synthesis, translocation and carbon reshuffling was made by measuring ${ }^{12} \mathrm{C}$ and ${ }^{13} \mathrm{C}$ fatty acids. The start of nitrogen starvation was considered as time 0 . All figures represent the average of two independent biological photobioreactor experiments. 

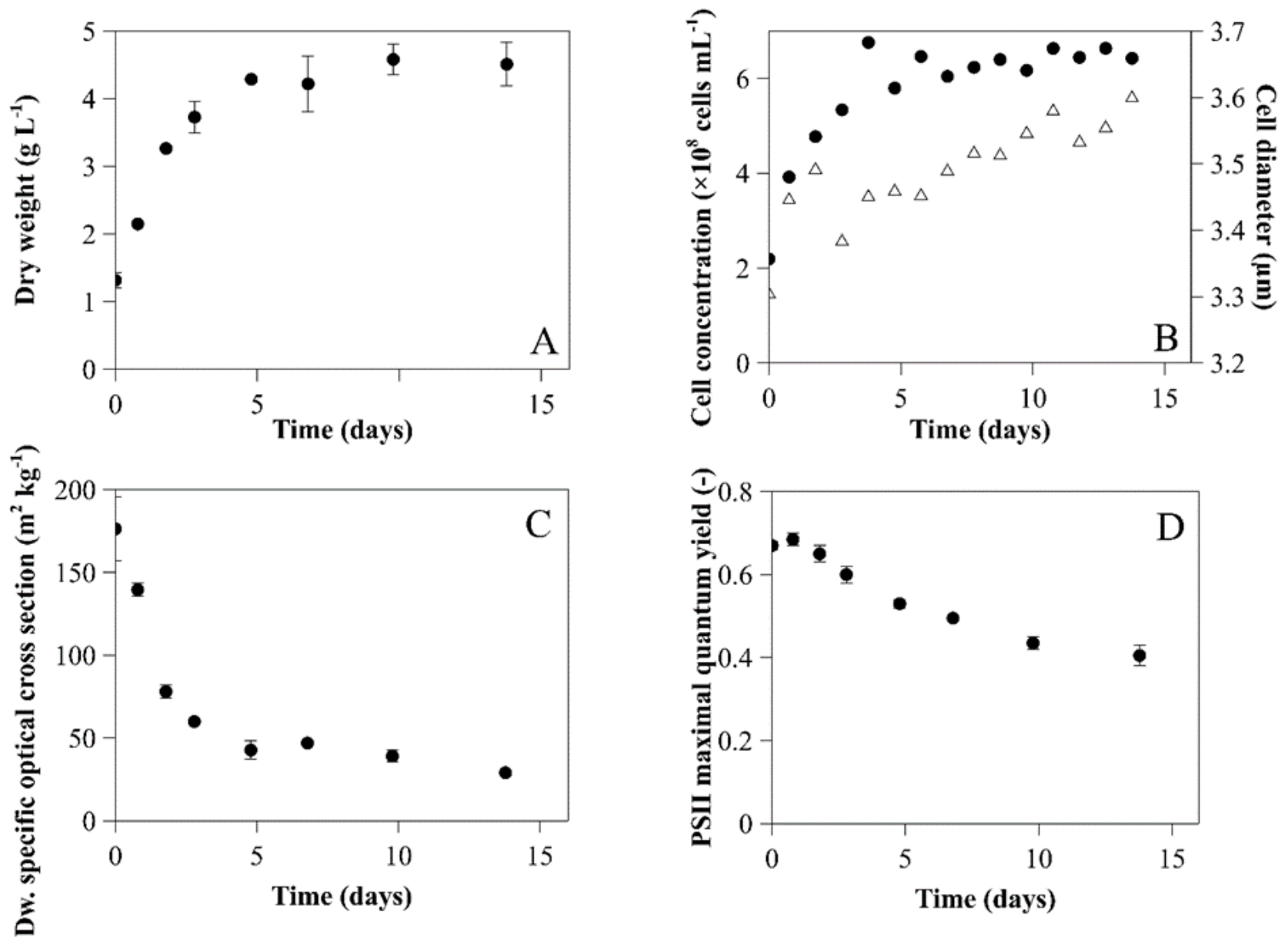

Figure 2.1. Average dry weight concentration $\left(\mathrm{g} \mathrm{t}^{-1}\right)(\mathrm{A})$, cell concentration (cells $\mathrm{L}^{-1}$ ) (circles) and diameter $(\mu \mathrm{m})$ (triangles) from one of the duplicate experiments (B), average dry weight-specific optical cross section $\left(\mathrm{m}^{2} \mathrm{~kg}^{-1}\right)$ (C) and average PSII maximal quantum yield (D) measured over time from the moment of nitrogen starvation. Error bars in A, C and D represent the minimum and maximum values of two biological reactor experiments $(n=2)$. 


\subsubsection{Biomass production and photosystem activity}

The nitrogen starvation started at a biomass concentration of $1.3 \pm 0.1 \mathrm{~g} \mathrm{~L}^{-1}$. The biomass concentration rapidly increased during the first 5 days up to $4.2 \pm 0.02 \mathrm{~g} \mathrm{~L}^{-1}$ and stabilized at $4.5 \pm 0.3 \mathrm{~g} \mathrm{~L}^{-1}$ at day 14 (Figure 2.1A). During this period the cells number increased 3 -fold from $2.2 \times 10^{8}$ to $6.6 \times 10^{8}$ cells per $\mathrm{mL}$ (Figure 2.1B), indication that on average the cells divided 1.5 times after nitrogen starvation until day 5. Pal et al., 2011 also observed that cells of Nannochloropsis sp. divide at least once during nitrogen starvation (Pal et al., 2011). The average cell diameter increased linearly from 3.3 to $3.6 \mu \mathrm{m}$ with increasing nitrogen starvation time (Figure 2.1B).

The dry weight-specific optical cross section rapidly decreased during the first 5 days of nitrogen starvation from $176 \pm 19$ to $29 \pm 1.7 \mathrm{~m}^{2} \mathrm{~kg}^{-1}$ (Figure $2.1 \mathrm{C}$ ). The PSII maximum quantum yield $\left(F_{v} / F_{m}\right)$ decreased from 0.67 to 0.40 (Figure 2.1D), indicating a reduced photosystem II activity. PSII maximum quantum yield is known to decrease during abiotic stress (Young and Beardall, 2003). Our findings are similar to a decrease from 0.60 to 0.49 found by Simionato et al. 2013 during 7 days of nitrogen starvation (Simionato et al., 2013). The decrease in PSII maximum quantum yield, dry weight-specific optical cross section and reduction in biomass productivity all point to a decrease in photosynthetic activity during nitrogen starvation.

\subsubsection{Fatty acid analysis using GC-FID}

Neutral triacylglycerol (TAGs) lipids were separated from the polar lipid fraction (PL) and the fatty acid composition of both fractions were analysed (as their FAMEs) during nitrogen starvation. The PL fraction will mainly consist of membrane lipids. The TAG and PL fractions were calculated as the sum of each fatty acids in that fraction. 

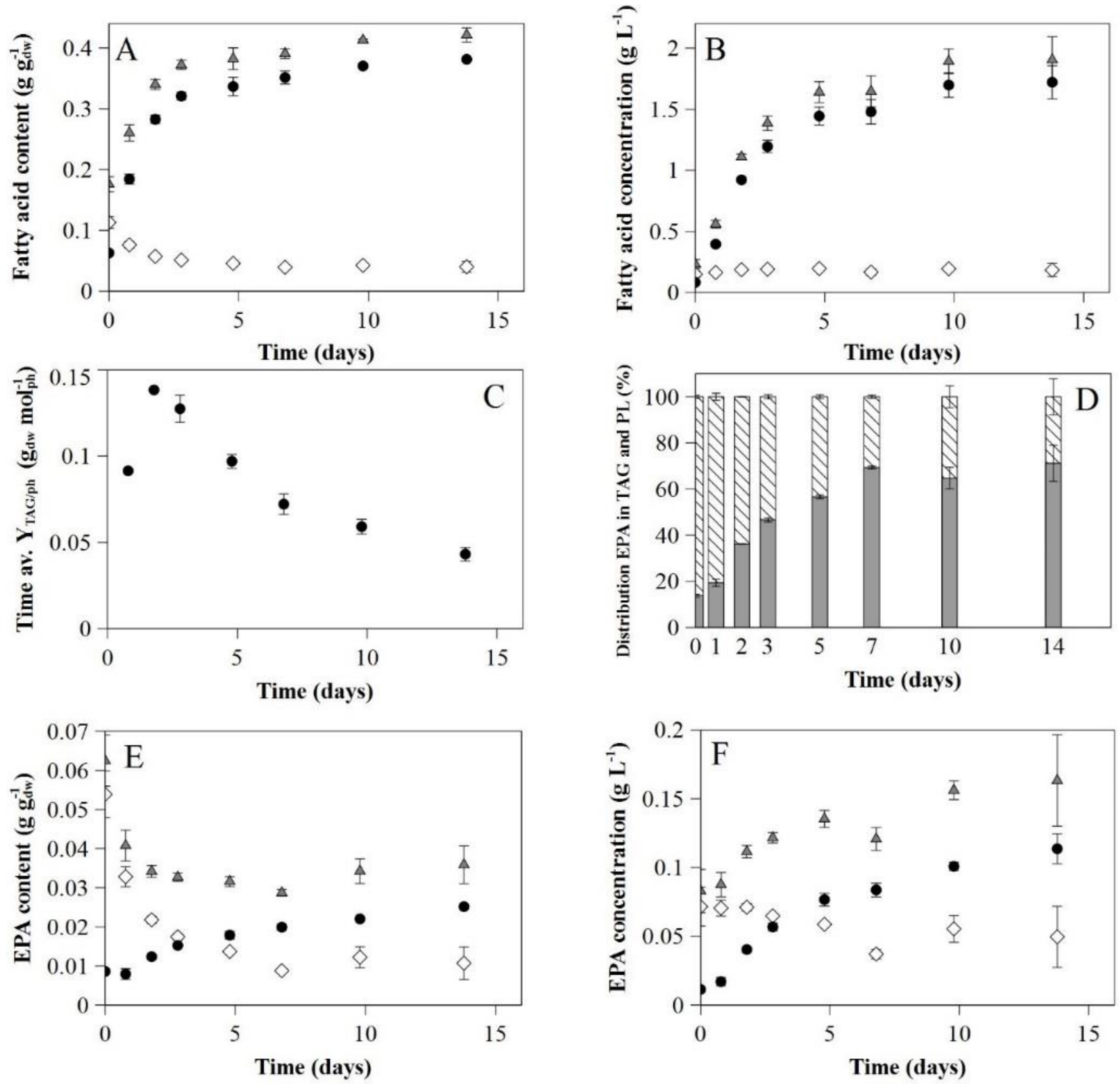

Figure 2.2. Average fatty acid content $\left(\mathrm{g} \mathrm{gdw}^{-1}\right)(A)$ and fatty acid concentration $\left(\mathrm{g} \mathrm{L}^{-1}\right)(\mathrm{B})$ in TAGs (circles), PL (diamonds) and total lipids (triangles; TAGs + PL). The average time-averaged TAG yield on light $\left(\mathrm{g}_{\mathrm{TAG}} \mathrm{mol}_{\mathrm{ph}}{ }^{-1}\right)$ (C). Average distribution of total EPA over the TAGs (grey bars) and PL (diagonal stripes) (D). The average content $\left(\mathrm{g} \mathrm{gdw}^{-1}\right)(\mathrm{E})$ and concentration $\left(\mathrm{g} \mathrm{L}^{-1}\right)$ (F) of EPA in both TAGs (circles), PL (diamonds) and total lipids (triangles). All from the start of nitrogen starvation. The error bars represent the minimum and maximum values of two biological photobioreactor experiments $(n=2)$. 
The total fatty acid content increased from 18 to $42 \%$ of the biomass dry weight in 14 days of nitrogen starvation (Figure 2.2A). This increase was mainly caused by the increase of the TAG fraction. The TAG concentration in the culture also rapidly increased 21-fold due to the increase in both TAG content and biomass concentration (Figure 2.2B). The maximal time-averaged TAG yield on light $(0.14 \pm$ $0.0004 \mathrm{~g}_{\mathrm{TAG}} \mathrm{mol}_{\mathrm{ph}}{ }^{-1}$, or $0.18 \pm 0.004 \mathrm{~g}_{\mathrm{TAG}} \mathrm{mol}_{\mathrm{ph}}{ }^{-1}$ when not corrected for the energy necessary for inoculum production) was achieved after 1 day of nitrogen starvation (Figure 2.2C). The PL content decreased within the first 3 days of nitrogen starvation from $0.11 \pm 0.01$ to $0.04 \pm 0.01 \mathrm{~g} \mathrm{gdw}^{-1}$. At day 14 of nitrogen starvation $90 \%$ of all fatty acids were present in the TAG fraction, similar to what was found for Nannochloropsis oculata (Tonon et al., 2002).

During nitrogen starvation the total cell EPA content expressed per total dry weight decreased from $0.06 \pm 0.01$ to $0.04 \pm 0.01 \mathrm{gepa}_{\mathrm{gdw}^{-1}}$ while EPA present in the TAG fraction increased from $0.01 \pm 0.001$ to $0.03 \pm 0.001 \mathrm{gepa}_{\mathrm{dw}}{ }^{-1}$. At the same time, EPA present expressed per total dry weight in the PL fraction decreased from $0.05 \pm 0.006$ to $0.01 \pm 0.004 g_{e p a} g_{d w}{ }^{-1}$ (Figure 2.2E). In this study the total EPA content before nitrogen starvation was thus slightly higher compared to $4.3 \%$ found in N. gaditana and 4.2 - 4.9\% in Nannochloropsis sp. (Camacho-Rodríguez et al., 2014; Hulatt et al., 2017).

In addition to the decrease in the algal EPA content upon nitrogen starvation, the EPA concentration in the bioreactor also changes due to the change in biomass concentration: from $82.9 \pm 15.7$ to $163.3 \pm 33.2 \mathrm{mg} \mathrm{L}^{-1}$. This increase thus represents de novo synthesis of EPA of $80.2 \mathrm{mg} \mathrm{L}^{-1}$ in total. The EPA concentration expressed per reactor volume, present in the TAG fraction increased from $11.4 \pm 1.7$ to $113.6 \pm 10.9$ $\mathrm{mg} \mathrm{L}^{-1}$, while in the $\mathrm{PL}$ it decreased from $71.5 \pm 14.1$ to $49.6 \pm 22.3 \mathrm{mg} \mathrm{L}^{-1}$ (Figure 2.2F).

At the start of nitrogen starvation, $13.8 \pm 0.6 \%$ of all EPA was present in the TAGs and it increased to $71 \pm 7.8 \%$ at day 14 of nitrogen starvation (Figure 2.2D). Similar results have been reported for $N$. oculata, in which $8 \%$ of EPA was present in TAG during the exponential phase and increased to $68 \%$ in the stationary phase (Tonon et al., 2002).

The total EPA in the culture increased during nitrogen starvation, indicating de novo synthesis of EPA. Nevertheless, the absolute increase in EPA present in TAG is larger 
than the absolute increase of EPA in the culture and therefore it could be hypothesized that EPA was partially transferred from the PL into TAG.

\subsubsection{Specific analysis of ${ }^{12} \mathrm{C}$ and ${ }^{13} \mathrm{C}$ fatty acids using accurate mass LC-MS}

By just measuring the fatty acid contents, no distinction can be made between possible de novo synthesis, degradation and translocation of fatty acids between the TAG and PL fractions. Therefore, the ${ }^{13} \mathrm{C} /{ }^{12} \mathrm{C}$ labelling patterns of the fatty acids were analysed. Because ${ }^{13} \mathrm{C}$ was the sole carbon source during the growth phase, virtually all microalgal carbon consisted of ${ }^{13} \mathrm{C}$ at the moment of nitrogen starvation (t0). $79 \%$ of all EPA at start of nitrogen starvation consisted only of ${ }^{13} \mathrm{C}$. During subsequent nitrogen starvation ${ }^{12} \mathrm{C}$ was used as carbon source. Consequently, all de novo synthesized fatty acids will consist of ${ }^{12} \mathrm{C}$ while fatty acids originating from the growth phase will fully consist of ${ }^{13} \mathrm{C}$. Fatty acids consisting of a mixture of ${ }^{12} \mathrm{C}$ and ${ }^{13} \mathrm{C}$ are synthesized by reshuffling ${ }^{13} \mathrm{C}$-carbon available in the cell at the onset of nitrogen starvation combined with ${ }^{12} \mathrm{C}$-carbon that is subsequently fixed de novo. The recycled ${ }^{13} \mathrm{C}$-carbon can originate from existing fatty acids but also from other biomass components and free ${ }^{13} \mathrm{CO}_{2}$ present in the cell prior to starvation.

At the onset of nitrogen starvation, the largest part of all fatty acids consisted only of ${ }^{13} \mathrm{C}$. Figure 2.3 shows the ${ }^{13} \mathrm{C},{ }^{12} \mathrm{C}$ and ${ }^{12} \mathrm{C} /{ }^{13} \mathrm{C}$ fatty acid concentrations of the four most abundant fatty acids, eicosapentaenoic acid (C20:5), palmitic acid (C16:0), palmitoleic acid (C16:1) and oleic acid (C18:1) in Nannochloropsis gaditana during nitrogen starvation. Upon subsequent nitrogen starvation, palmitic acid, palmitoleic acid and oleic acid in the TAG fraction were mainly produced by de novo synthesis from ${ }^{12} \mathrm{C}$ sources only (carbon chains consisting of ${ }^{12} \mathrm{C}$ only) and de novo synthesis combined with ${ }^{13} \mathrm{C}$-carbon reshuffling (carbon chain consisting of a mix of ${ }^{12} \mathrm{C}$ and ${ }^{13} \mathrm{C}$ ). In contrast, only small amounts of fatty acids were newly synthesized in the PL and mainly originated from de novo synthesis from ${ }^{12} \mathrm{C}$-carbons only. 

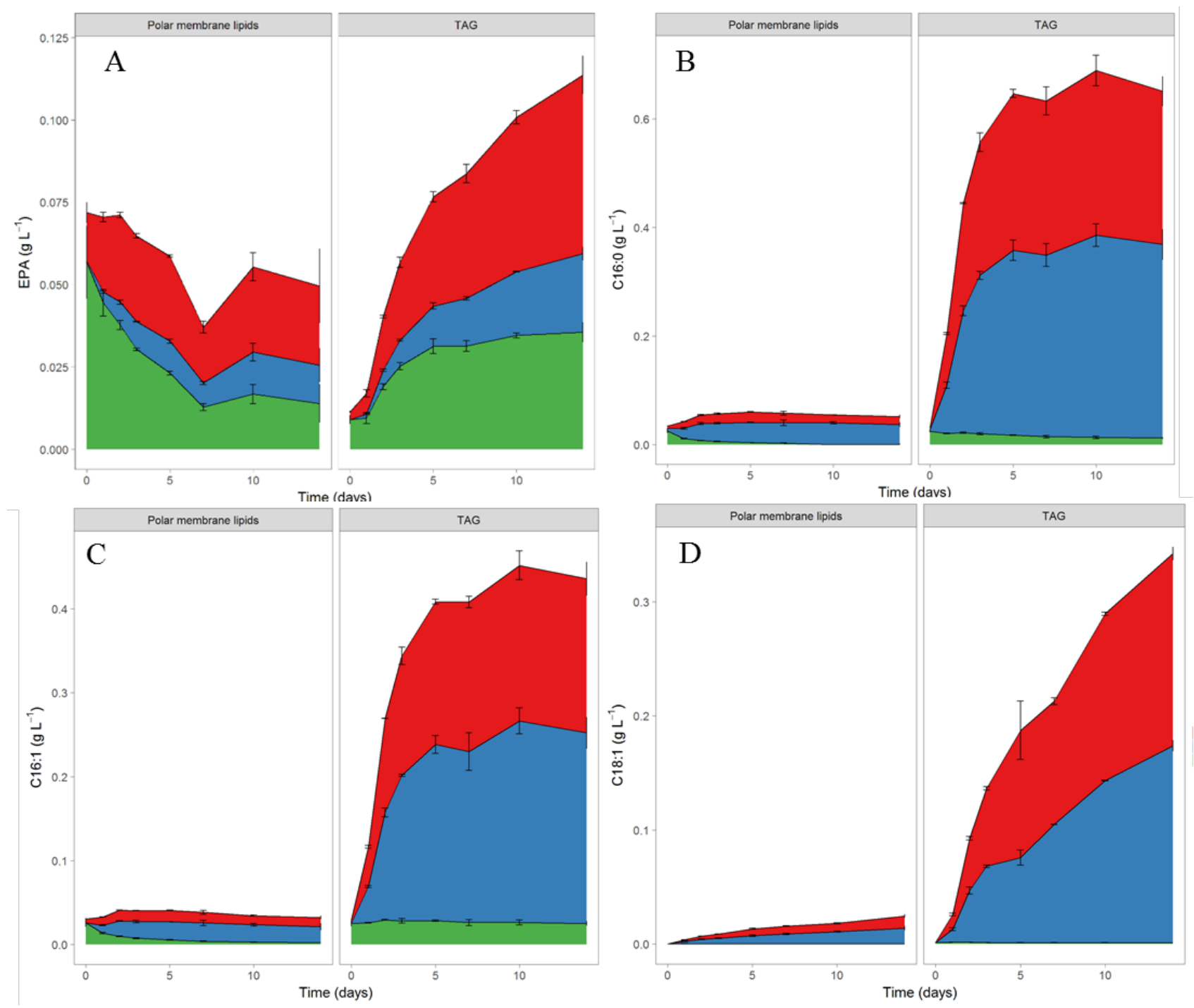

Figure 2.3. Average fatty acids $\left(\mathrm{mg} \mathrm{L}^{-1}\right)$ consisting of only ${ }^{13} \mathrm{C}$ (green), only ${ }^{12} \mathrm{C}$ (blue) and a mix of ${ }^{12} \mathrm{C}$ and ${ }^{13} \mathrm{C}$ (red) at different times during nitrogen starvation in PL and TAGs. Eicosapentaenoic acid (C20:5) (A), palmitic acid (C16:0) (B), palmitoleic acid (C16:1) (C) and oleic acid (C18:1) (D). Error bars represent the minimum and maximum values of two biological experiments $(n=2)$. 
EPA consisting of only ${ }^{13} \mathrm{C}$ decreased in the PL fraction and increased in the TAG fraction (Figure 2.3). The absolute EPA decrease in the PL fraction, i.e. $0.04 \mathrm{~g} \mathrm{~L}^{-1}$, is similar to its absolute increase in the TAG fraction, i.e. $0.03 \mathrm{~g} \mathrm{~L}^{-1}$. This result provide evidence for translocation of the complete EPA molecule from PL to TAG, since it is unlikely that all 20 carbon atoms of EPA were completely made from recycled ${ }^{13} \mathrm{C}$-carbon. Of all the EPA present in TAG after 14 days of nitrogen starvation, $23 \%$ originated of translocation from PL to TAG. This is the first time showing actual translocation of intact EPA from PL into TAG in microalgae. In addition to translocation, in both PL and TAG fractions EPA was de novo synthesized from both ${ }^{13} \mathrm{C}$-carbon recycled within the nitrogen starved cell and de novo synthesis from ${ }^{12} \mathrm{C}$-carbon. In the TAG fraction, after 14 days of nitrogen starvation $21 \%$ of all EPA was completely made de novo from ${ }^{12} \mathrm{C}$-carbon, $46 \%$ was made from ${ }^{13} \mathrm{C}$-carbon recycled within the cell together with de novo ${ }^{12} \mathrm{C}$-carbon, $23 \%$ originated from translocation of intact ${ }^{13} \mathrm{C}$-EPA and $10 \%$ was already present at the start of nitrogen starvation. One enzyme which could be responsible for the observed EPA transfer into TAG is phospholipid: diacylglycerol acyltransferase (PDAT). This gene has been identified in the genome of Nannochloropsis gaditana (Radakovits et al., 2012) and it would be interesting to study its transcriptional and protein levels during nitrogen starvation. It is interesting to note that mainly EPA was translocated, relative to the other fatty acid species, suggesting that the enzyme responsible for the translocation has a relative high affinity for EPA as a substrate.

\subsection{CONCLUSIONS}

Using ${ }^{13} \mathrm{C}$ isotope labelling, we provide conclusive evidence for translocation of intact EPA carbon chains from the PL into the neutral TAGs upon nitrogen starvation of microalgae. Next to translocation, EPA was also synthesized both completely de novo and from carbon reshuffling within the cell. In contrast, to EPA, palmitic acid, palmitoleic acid and oleic acid were hardly translocated and mainly synthesized de novo and from carbon reshuffling, indicating that the enzyme responsible for translocation of intact fatty acids has a high affinity towards EPA relative to other fatty acids. Future research will focus on elucidating the biochemical pathways active in fatty acid translocation and de novo synthesis, in order to gain better insight into the regulation of EPA accumulation in nitrogen starved microalgae. This knowledge can potentially be used to produce EPA-rich TAG from Nannochloropsis. 


\subsection{Supplement}

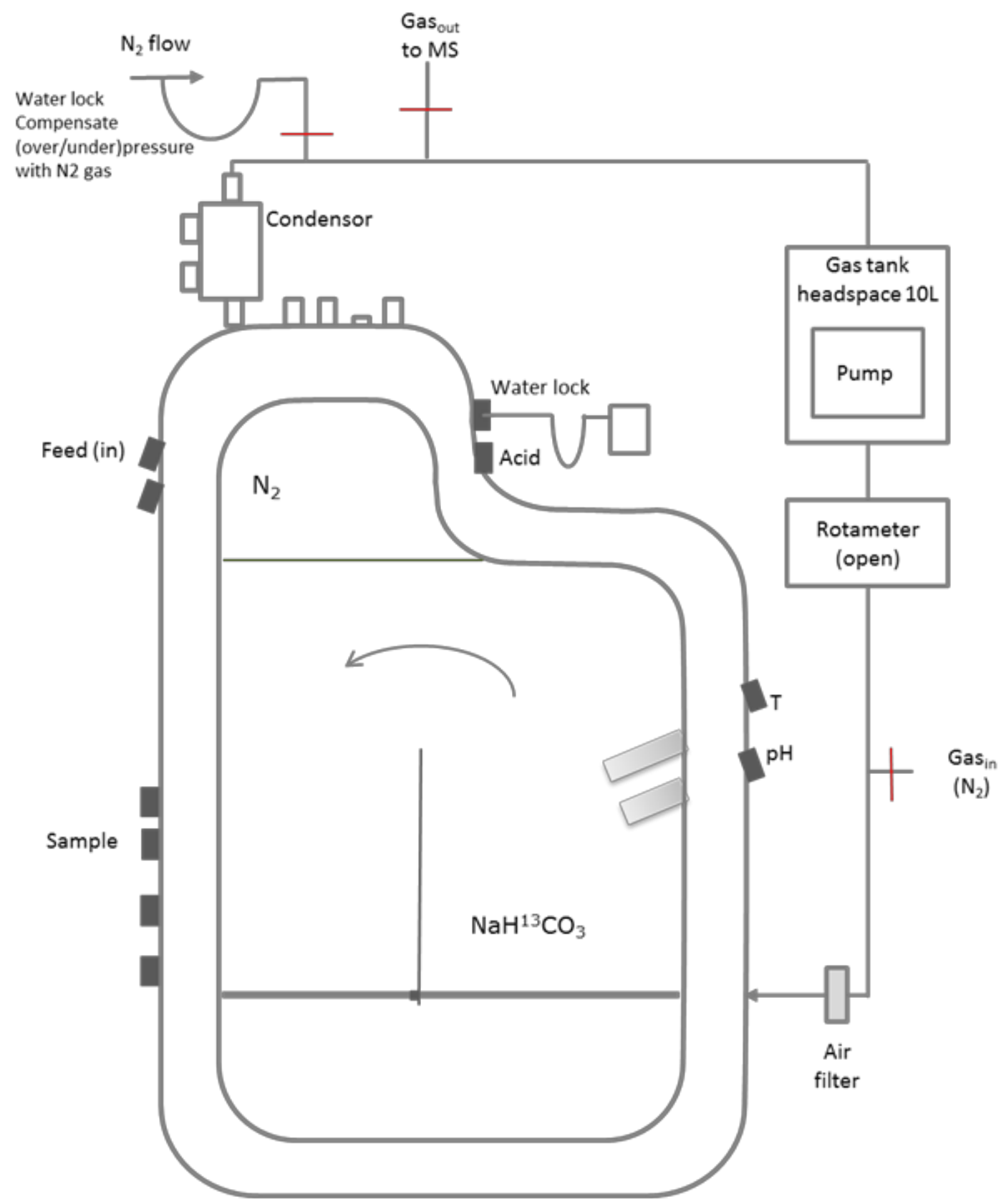

Figure S2.4. Schematic overview flat panel photobioreactor setup for the growth phase. 


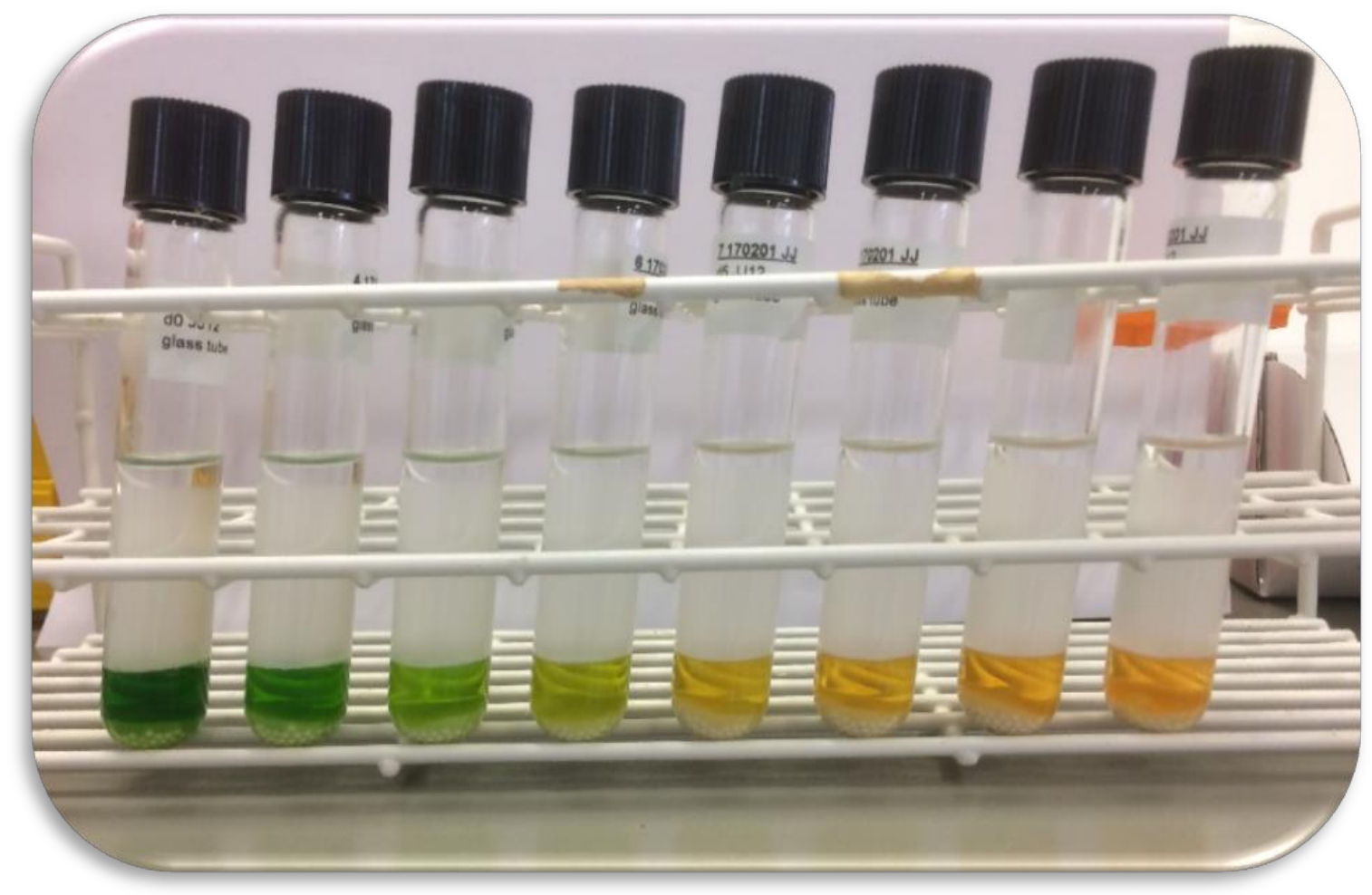

Colour change of time series during lipid extraction. 


\section{Chapter 3}

Expression levels of genes involved in TAG and polyunsaturated fatty acid synthesis of Nannochloropsis gaditana during short- and longterm nitrogen starvation

This chapter is prepared to submit for publication as:

JH Janssen, J Spoelder, JJ Koehorst, PJ Schaap, RH Wijffels and MJ Barbosa.

Expression level of genes involved in TAG and polyunsaturated fatty acid synthesis of Nannochloropsis gaditana during short- and long-term nitrogen starvation 


\section{ABSTRACT}

Microalgae can be used as a source of triacylglycerol (TAG) and the omega-3 fatty acid eicosapentaenoic acid (EPA). Insight in TAG and EPA production and regulation are needed to improve productivity. Nitrogen starvation induces TAG accumulation. Previous research showed that during nitrogen starvation in Nannochloropsis gaditana, EPA was translocated from the polar lipids to TAG and de novo synthesized. Expression levels of genes involved in de novo TAG synthesis pathway and fatty acid translocation were measured during the first hours of nitrogen starvation and over a longer starvation period. Furthermore, the genes involved in fatty acid elongation and desaturation were studied. One phospholipid: diacylglycerol acyltransferase (PDAT) gene involved in translocation of fatty acids from membrane lipid to TAG was upregulated. In addition, several lipases showed increased transcriptional levels, suggesting these enzymes might be responsible for translocation of EPA accumulation in TAG. Most desaturases and elongases involved in de novo EPA synthesis were downregulated except for $\Delta 9$ desaturase was upregulated which correlated with the increase in oleic acid. Because there are many hypothetical genes, improvements in annotation are needed for a better understanding of the pathways. 


\subsection{INTRODUCTION}

Microalgae are a sustainable source of lipids which can be used for food, feed and fuel. Fatty acids can accumulate in triacylglycerol (TAG) lipid droplets and in membrane lipids. Nannochloropsis gaditana is a microalga known for its large TAG accumulation during nitrogen starvation and the production of omega-3 fatty acid eicosapentaenoic acid (EPA). The genome of $N$. gaditana has been sequenced (Corteggiani Carpinelli et al., 2014; Radakovits et al., 2012), which enables transcriptomic analysis by measuring the expression levels using RNA sequencing (RNA-seq). The expression levels give information about the activity of the genes in the studied pathways.

In microalgae TAG can be produced via two pathways; the acyl-CoA dependent Kennedy pathway and the acyl-CoA independent pathway. These pathways start with the fatty acids synthesis by the fatty acid synthase (FAS) complex. The FAS complex produces $\mathrm{C} 16: 0, \mathrm{C} 18: 0$ and C18:1 fatty acids as acyl-CoA. These can be used in the acyl-CoA dependent Kennedy pathway to produce TAG but also be used as substrate for polyunsaturated fatty (PUFA) synthesis. An overview of the most important steps in the TAG synthesis pathways are given in Figure 3.1. 


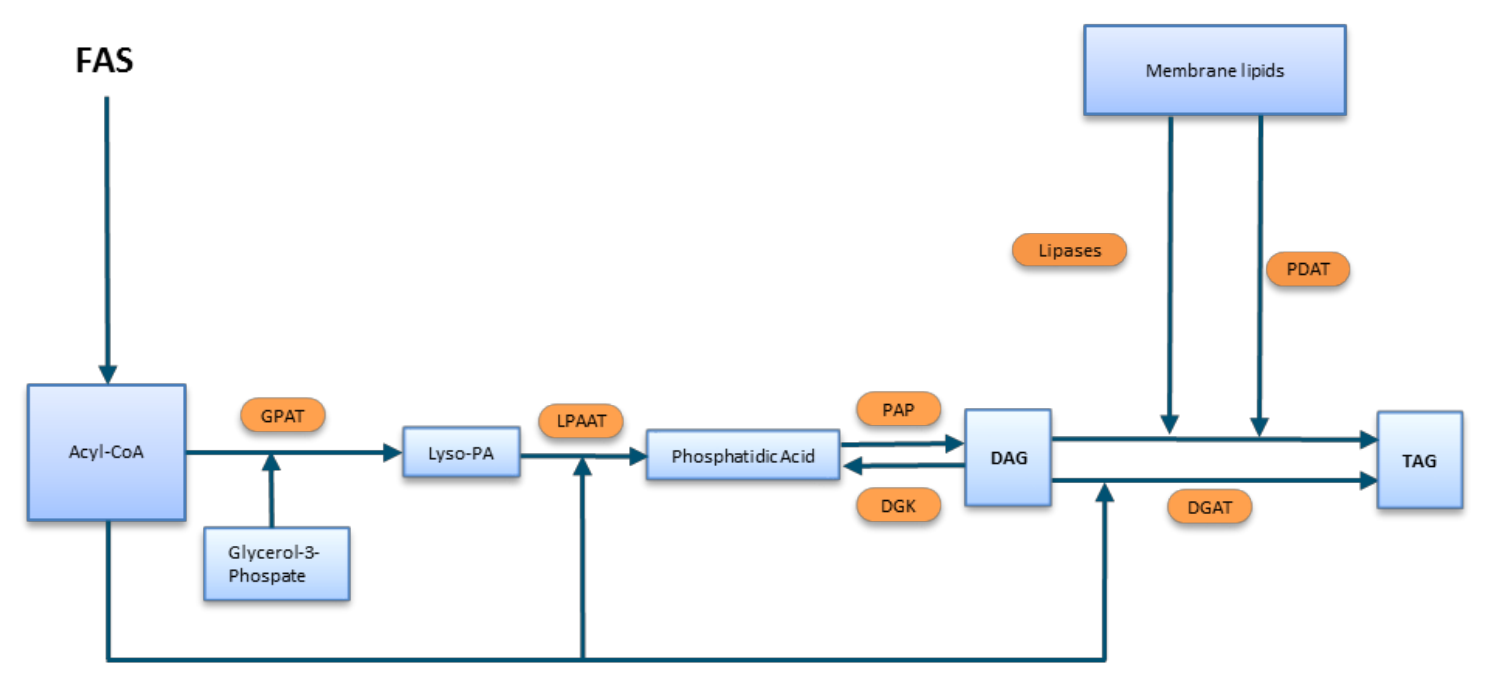

Figure 3.1. Proposed simplified TAG synthesis pathways of Nannochloropsis gaditana. GPAT: glycerol-3-phosphate acyltransferase, Lyso-PA: lysophosphatidic acid, LPAAT: lysophosphatidic acid acyltransferase, PAP: phosphatidic acid phosphatase, DGK: diacylglycerol kinase, DAG: diacylglycerol, DGAT: diacylglycerol acyltransferase, TAG: triacylglycerol and PDAT: phospholipid: diacylglycerol acyltransferase. Adapted from (Banerjee et al., 2017; Ma et al., 2016; Radakovits et al., 2012).

\subsubsection{TAG synthesis via acyl-CoA dependent pathway}

The acyl-CoA synthesized by FAS can be transferred to glycerol-3-phosphate by glycerol-3-phosphate acyltransferase (GPAT) to yield lysophosphatidic acid (LysoPA). The addition of another acyl chain to Lyso-PA by lysophosphatidic acid acyltransferase (LPAAT) produces phosphatidic acid (PA). The dephosphorylation of PA by phosphatidic acid phosphatase (PAP) yields diacylglycerol (DAG). The last step is the acylation of DAG to triacylglycerol (TAG) by diacylglycerol acyltransferase (DGAT). Nannochloropsis has one or two gene copies of the DGAT-1 family and 11 gene copies of DGAT-2 gene family. With 11 gene copies of DGAT-2 they have the highest gene dose of DGAT-2 among known genomes (Alboresi et al., 2016; Wang et al., 2014). 


\subsubsection{TAG synthesis by the acyl-CoA independent pathway}

Next to TAG synthesis via the acyl-CoA dependent pathway, TAG can also be synthesized by an acyl-CoA independent pathway. In this pathway fatty acids originate from the membrane lipids and can be translocated to TAG by for example phospholipid: diacylglycerol acyltransferase (PDAT) or lipases. Previous research showed that $23 \%$ of the EPA accumulated in TAG originated from translocation of intact EPA from the polar lipids to the TAG upon nitrogen starvation (Chapter 2). This translocation seemed to have a high specificity for EPA, as mainly EPA was translocated.

The PDAT enzyme isolated from the microalgae Chlamydomonas reinhardtii showed phospholipid and galactolipid diacylglycerol transferase activity, DAG: DAG transacylase activity (producing TAG and monoacylglycerol (MAG)) and showed lipase activity with broad substrate specificity (Yoon et al., 2012). Therefore, PDAT can be responsible for the production of TAG in different ways, including the translocation of membrane lipids to TAG. An insertional mutant of PDAT in C. reinhardtii showed $25 \%$ less TAG accumulation showing the relevance of this pathway for TAG synthesis during nitrogen starvation (Boyle et al., 2012).

Another possible way to translocate fatty acids from membranes to TAG in lipid bodies is by lipases which free the fatty acids allowing translocation to TAG. For $C$. reinhardtii and $N$. oceanica multiple lipases were upregulated upon nitrogen starvation (Li et al., 2014; Miller et al., 2010).

\subsubsection{EPA synthesis}

The EPA synthesis starts after the production of palmitic acid (C16:0) and stearic acid (C18:0) in FAS. EPA is synthesized by sequential desaturation and elongation steps performed by desaturases and elongases, respectively. The proposed EPA biosynthesis pathway for Nannochloropsis shows multiple pathways (Figure 3.2). 


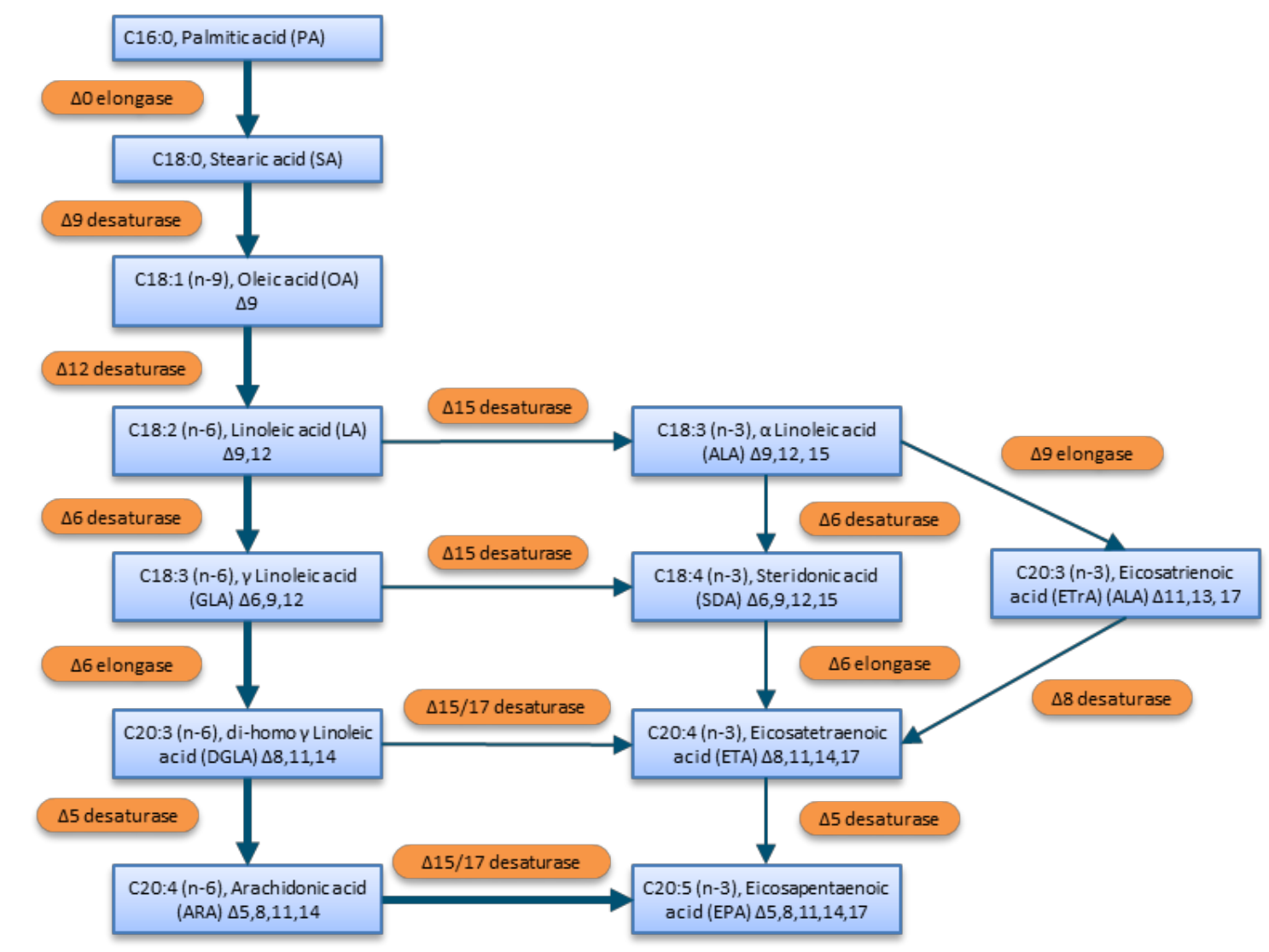

Figure 3.2. Proposed EPA synthesis pathway for Nannochloropsis adapted from (Schneider and Roessler, 1994; Vieler et al., 2012).

After the first desaturation step by $\Delta 9$ desaturase oleic acid (C18:1 n-9) is formed. The second desaturation step by $\Delta 12$ desaturase results in the formation of linoleic acid (C18:2 n-6). From linoleic acid two main pathways can be used for EPA synthesis: the omega- 6 and the omega-3 pathway. Based on the measured intermediate fatty acids by Schneider and Roessler 1994, the omega- 6 pathways may be predominate in Nannochloropsis and is therefore shown with bold arrows (Schneider and Roessler, 1994) (Figure 3.2). The produced EPA can be used in TAG synthesis or membrane lipids synthesis. One hypothesized role of TAG is to act as reservoir for plastid PUFA under stress conditions (Cohen et al., 2000; Khozin-Goldberg et al., 2005).

The aim of this research is to get more insight in the TAG and EPA production pathways in Nannochloropsis gaditana during nitrogen starvation by analysing transcriptomic changes in time. Previous research showed that upon nitrogen starvation $23 \%$ of EPA present in TAG was intact translocated from the polar lipids in 
N. gaditana, $21 \%$ was de novo synthesized and $46 \%$ resulted from synthesis via carbon recycled within the cell with newly incorporated carbon (Chapter 2 ). In this research we want to get more insight in the translocation of fatty acid during nitrogen starvation and therefore studied the genes involved in the acyl-CoA dependent pathway and acyl-CoA independent pathway (PDAT and lipases). In addition, since EPA was also shown to be made de novo after nitrogen starvation, genes involved in the EPA synthesis were analysed. To be able to compare the transcriptome analysis data to the data from previous research where translocation was measured via ${ }^{13} \mathrm{C}$ labelling, the same experimental setup was used. $N$. gaditana was grown in two-phase batch cultivation where a growth phase was followed by a nitrogen starvation phase. Gene expression was analysed over a short (hours) and a longer time period (days), to be able to analyse the immediate transcriptional changes at the onset of nitrogen starvation and on a longer time period. The transcriptional expression levels during nitrogen starvation were compared to nitrogen replete conditions. In addition to transcriptional expression levels the fatty acid composition and content in the TAG and polar lipids were measured. 


\subsection{MATERIALS AND METHODS}

\subsubsection{Strain, cultivation medium and pre-cultivation}

The microalgae Nannochloropsis gaditana CCFM-01 was obtained from the Microalgae Collection of Fitoplancton Marino S.L. Pre-cultivation and growth medium used were as described in Chapter 2.2.1.

\subsubsection{Photobioreactor and experimental setup}

The experiments were performed in an aseptic, heat-sterilized, flat-panel, airlift-loop photobioreactor (Labfors 5 Lux, Infors HT, Switzerland, 2010) with a reactor depth of $0.02 \mathrm{~m}$. The experimental setup was used as described in Chapter 2.2.4, with the exception that during the growth phase the same experimental setup was used as during the nitrogen starvation phase, therefore without gas recirculation. For the growth and nitrogen starvation mixing was done by sparging $1 \mathrm{~L} \mathrm{~min}^{-1}$ of air mixed with $2 \% \mathrm{CO}_{2}$.

\subsubsection{Offline measurements of the culture and lipid analysis}

The measurements of dry weight and cell concentration were measured daily from the start of nitrogen starvation according to Chapter 2.2.5. The lipid analysis and TAG yield on light calculations were performed according to Chapter 2.2.7.

\subsubsection{RNA-sequencing}

At $0,0.1,0.3,0.8,1,2,3,5,7$ and 14 days biomass samples for RNA-sequencing were taken. The biomass samples were directly kept on ice and centrifuged for 5 minutes $\left(4700 \mathrm{~g}, 0^{\circ} \mathrm{C}\right)$. The cell pellets were immediately frozen in liquid nitrogen and stored at $-20^{\circ} \mathrm{C}$. RNA was extracted using Maxwell ${ }^{\circledR} 16$ LEV simplyRNA Cells Kit following the standard protocol. Extracted RNA was tested for quality using an Experion RNA Analysis Kit (BIO-RAD). RNA samples were stored at $-80^{\circ} \mathrm{C}$ and sequenced by Novogene (Illumina PE150).

The genomic annotation (GFF3) and corresponding genomic sequence (FASTA) of Nannochloropsis gaditana were converted into a semantic framework using SAPP according to the GBOL ontology (Dam et al., 2017; Koehorst et al., 2017). Each RNAseq dataset was mapped using the Transcriptomics module using STAR 2.5 as 
the read mapping software (Dobin et al., 2013). The expression for each gene for all conditions were converted into a data frame for differential analysis using DESeq2 (Love et al., 2014). Iteratively, differential expression for all comparisons between the different time points where analysed.

The $\log _{2}$ fold change (LFC) for each time point in the starvation phase was calculated compared to the expression levels at the nitrogen replete phase. A principal component analysis (PCA) was performed to compare the biological replicates (Figure S3.10). Most replicates of the same time point are closely aligned, showing good reproducibility. The samples of day 0.3 were less well aligned, but since only duplicates were available, both samples were included.

Genes were considered significant differentially expressed when the p-value adjusted for false positives (padj) was below or equal to 0.05 and minimum of two for these time points were upregulated or downregulated LFC of 1.5 to 2, or at least one time point was upregulated or downregulated minimal LFC of 2 as described by (Li et al., 2014). 


\subsection{RESULTS AND DISCUSSION}

Results on biomass concentration, photosynthetic activity and lipids are shown from the start of nitrogen starvation and are the average of two biological photobioreactor experiments with the error bars showing the absolute deviation between these duplicates.

\subsubsection{Biomass concentration and photosystems}

The biomass concentration rapidly increased from $0.92 \pm 0.12$ to $4.9 \pm 0.36$ $\mathrm{g} \mathrm{L}^{-1}$ during 14 days of nitrogen starvation (Figure 3.3A). The cell concentration increased from $2.43 \pm 0.15 \times 10^{8}$ to $8.23 \pm 0.86 \times 10^{8}$ cells $\mathrm{mL}^{-1}$ during this period (Figure $3 \mathrm{~B}$ ), meaning that on average cells divided 1.5 times after nitrogen starvation. These growth results were similar to those obtained in Chapter 2 (Figure S3.8).
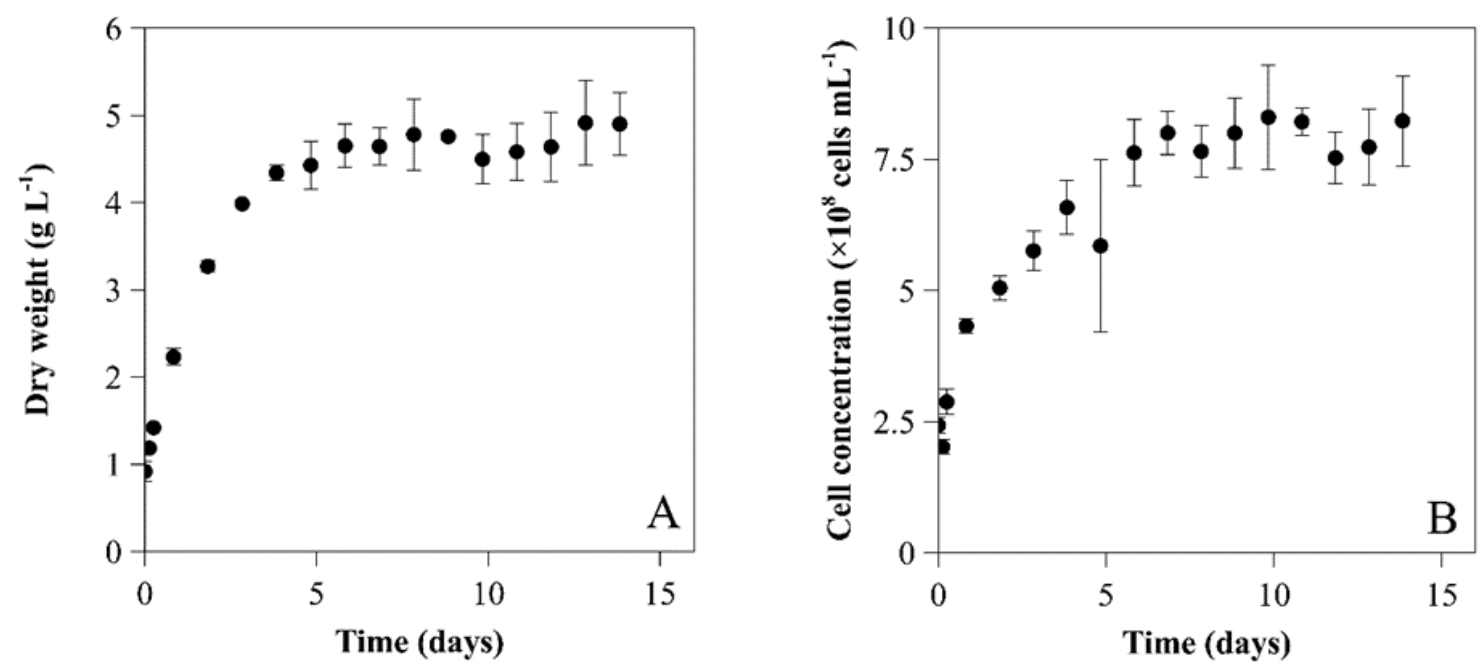

Figure 3.3. Average dry weight concentration $\left(\mathrm{g} \mathrm{L}^{-1}\right)(\mathrm{A})$ and average cell concentration (cells $\mathrm{mL}^{-1}$ ) (B) from the moment of nitrogen starvation. The error bars show the absolute deviation between two biological photobioreactor experiments $(n=2)$.

\subsubsection{Fatty acid accumulation}

Fatty acid accumulation in TAG and polar lipid fractions was measured during nitrogen starvation. 

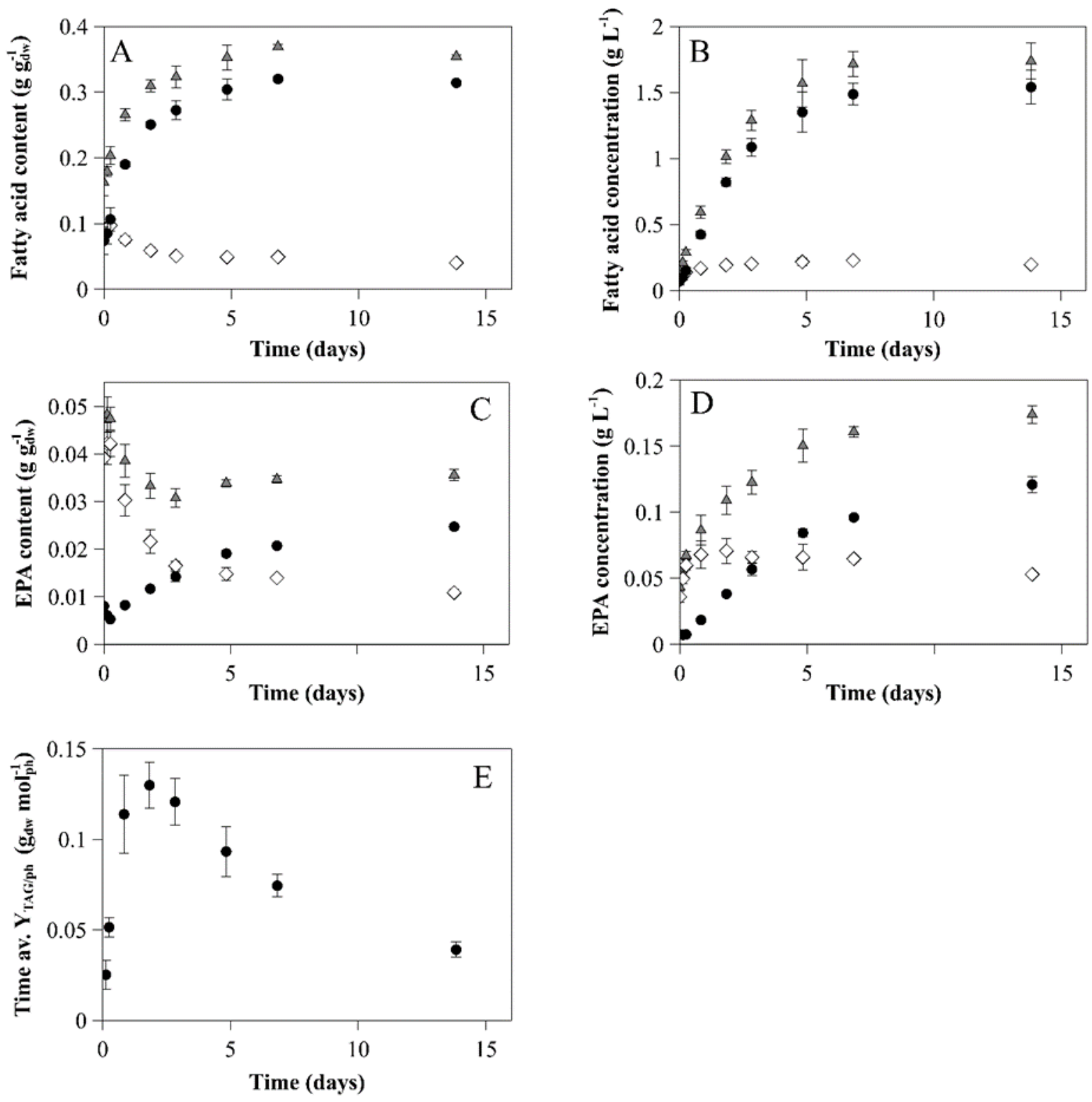

Figure 3.4. Average fatty acid content $\left(\mathrm{g} \mathrm{gdw}^{-1}\right)(\mathrm{A})$ and fatty acid concentration ( $\mathrm{g} \mathrm{L}^{-1}$ ) (B) in TAGs (circles), polar lipids (PL) (diamonds) and total lipids (triangles, TAG + PL). The average EPA content $\left(\mathrm{g} \mathrm{gdw}^{-1}\right.$ ) (C) and EPA concentration ( $\mathrm{g} \mathrm{L}^{-1}$ ) (D) in TAG (circles), polar lipids (diamonds) and total lipids (triangles, TAG $+\mathrm{PL}$ ). The average time-averaged TAG yield on light $\left(\mathrm{g}_{\mathrm{TAG}} \mathrm{mol}_{\mathrm{ph}}{ }^{-1}\right)$ including correction for energy necessary for inoculum production (E). Time zero corresponds to the start of nitrogen starvation. The error bars show the absolute deviation between two biological photobioreactor experiments $(n=2)$. 
The TAG content expressed per biomass increased from $0.07 \pm 0.02$ to $0.31 \pm 0.003$ $\mathrm{g} \mathrm{gdw}^{-1}$ during the 14 days of nitrogen starvation (Figure 3.4A). The polar lipid content expressed per biomass decreased from $0.09 \pm 0.001$ to $0.04 \pm 0.001 \mathrm{~g} \mathrm{~g}_{\mathrm{dw}^{-1}}$ after 14 days of nitrogen starvation (Figure $4 \mathrm{~A}$ ). The polar lipids mainly consist of membrane lipids. The polar lipid species monogalactosyl diacylglycerol (MGDG), digalactosyl diacylglycerol (DGDG), phosphatidylcholine (PC), phosphatidylinositol (PI) and phosphatidylglycerol (PG) are lipid constituents of photosynthetic membranes and have been reported to decrease during nitrogen starvation in Nannochloropsis gaditana (Simionato et al., 2013), in accordance with our results.

The increase in TAG content and biomass concentration resulted in an increase in TAG concentration in the reactor from $0.07 \pm 0.03$ to $1.54 \pm 0.13 \mathrm{~g} \mathrm{~L}^{-1}$ in 14 days of nitrogen starvation (Figure 3.4B). In the same period, the polar membrane lipid concentration increased from $0.08 \pm 0.01$ to $0.20 \pm 0.01 \mathrm{~g} \mathrm{~L}^{-1}$ (Figure $3.4 \mathrm{~B}$ ). This shows that, although the polar lipids expressed per biomass decreased, the total amount increased and part of the polar lipids was made de novo during nitrogen starvation.

The maximal time-averaged TAG yield on light was $0.13 \pm 0.01 \mathrm{~g}_{\mathrm{TAG}} \mathrm{mol}_{\mathrm{ph}}{ }^{-1}$ after 1 day of nitrogen starvation $\left(0.16 \pm 0.02 \mathrm{~g}_{\mathrm{TAG}} \mathrm{mol}_{\mathrm{ph}}{ }^{-1}\right.$ after 2 days when not corrected for energy necessary for inoculum) (Figure 3.4E).

The EPA content present in the TAG lipid fraction expressed per dry weight increased and decreased in the polar lipid fraction resulting in an overall decrease in EPA content (Figure 3.4C).

The EPA concentration present in the TAG and polar lipid fraction increased due to the increase in biomass concentration (Figure 3.4D). Resulting in a total increase in EPA concentration from $0.043 \pm 0.005$ to $0.174 \pm 0.007 \mathrm{~g} \mathrm{~L}^{-1}$.

In general, the largest changes in fatty acids content in TAG and PL, TAG yield and EPA content occurred within the first three days of nitrogen starvation. Results for fatty acids were similar to previous results under identical conditions (Figure S3.9) (Chapter 2). It was proven in that research that under these growth condition EPA accumulated in TAG was partly made de novo during nitrogen starvation, as also shown here by the increase in EPA concentration in the reactor. On the other hand, $23 \%$ of the EPA present in TAG was intact translocated from the polar lipid to the TAG during nitrogen starvation (Chapter 2). Another fraction of EPA in TAG was made from carbon recycled within the cell with newly incorporated carbon (Chapter 2). To 
study the accumulation pathways involved in TAG accumulation, the expression levels of genes involved in the acyl-CoA dependent and independent TAG synthesis pathways were analysed.

\subsubsection{Differential expression genes}

From the 10486 genes annotated in the genome 10105 (96\%) genes were identified in the RNA-sequencing data (Corteggiani Carpinelli et al., 2014). From the identified genes, 6946 (68\%) genes were significantly differential expressed based on the set constraints (Li et al., 2014). Within the differentially expressed genes 2908 (42\%) were annotated as hypothetical or unknown protein. In this research, we focussed on the genes involved in TAG metabolism and EPA synthesis.

\subsubsection{Genes involved in fatty acid synthesis}

Genes involved in the fatty acid biosynthesis showed no major changes in expression, even though the lipid cell content increased during nitrogen starvation. The FAS complex was mostly downregulated upon nitrogen starvation as previously reported for N. gaditana and N. oceanica (Corteggiani Carpinelli et al., 2014; Li et al., 2014). Although the pathway was not upregulated, it remained active since de novo fatty acid synthesis was shown under these growth conditions (Chapter 2).

\subsubsection{TAG synthesis via acyl-CoA dependent pathway}

Since $N$. gaditana accumulated TAG upon nitrogen starvation, the expression level of the genes involved in the acyl-CoA dependent TAG pathway were analysed (Figure 3.5). The $\log _{2}$ fold change (LFC) during nitrogen starvation was shown for the identified genes involved in this pathway. 


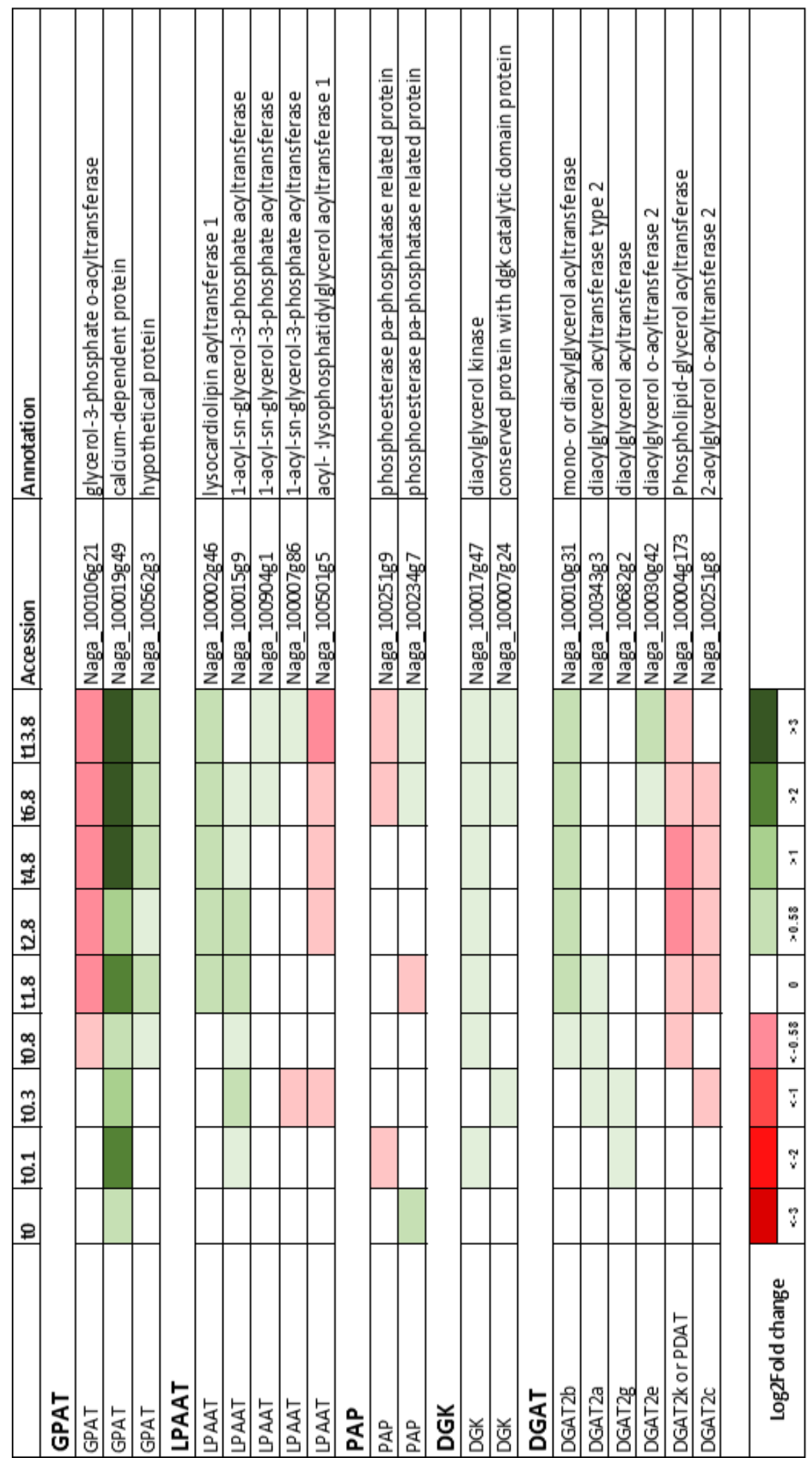

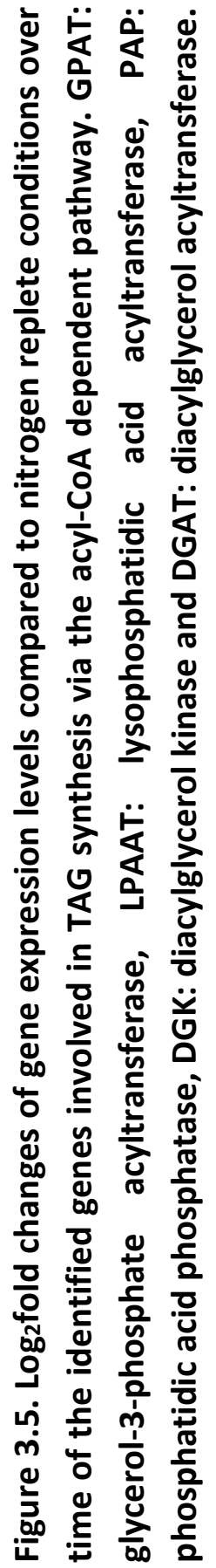


The first enzyme involved in TAG synthesis via the acyl CoA-dependent pathway is glycerol-3-phosphate acyltransferase (GPAT) which produces lysophosphatidic acid from glycerol-3-phosphate and acyl-CoA. Three genes identified as GPAT were differentially expressed compared to nitrogen replete conditions (Alboresi et al., 2016; Corteggiani Carpinelli et al., 2014). Two genes (Naga_100019g49 and Naga_100562g3) were upregulated and one was downregulated (Naga_100106g21) during nitrogen starvation (Figure 3.5). Similar results were found for Nannochloropsis oceanica where one GPAT was upregulated and another one was downregulated (Li et al., 2014). GPAT (Naga_100106g21) was downregulated 1.5-fold at 0.8 day and decreased to 2.4-fold from 1.8 days onward. The other GPAT gene (Naga_100562g3) was upregulated two-fold from 0.8 day onward. The largest increase up to 24-fold was shown in GPAT (Naga_100019g49) which was hardly present in nitrogen replete conditions and activated upon nitrogen starvation.

The next step in TAG synthesis is the formation of phosphatidic acid by lysophosphatidic acid acyltransferase (LPAAT). Five identified LPAAT genes (Alboresi et al., 2016; Dolch et al., 2017) were differentially expressed (Naga_100002g46, Naga_100015g9, Naga_100904g1, Naga_100007g86 and Naga_100501g5). LPAAT (Naga_100015g9) was upregulated 1.5 to 2.6 -fold consistently from 0.1 day onward and another LPAAT gene (Naga_100002g46) was upregulated from day 1.8 onward 2 to 2.5-fold. On the other hand, LPAAT (Naga_100501g5) was 1.5-fold downregulated at 0.3 day and from 3 days onward 2-fold downregulated.

The following step in the Kennedy pathway is the formation of DAG by phosphatidic acid phosphatase (PAP). One of two PAP genes (Naga_100234g7) was 2-fold upregulated at the start of nitrogen starvation and 1.5-fold at days 7 and 14 . The second PAP gene (Naga_100251g9) was downregulated 1.5 -fold at 0.3, 7 and 14 days of nitrogen starvation. Similar results, have been reported, in $N$. oceanica where one putative PAP gene was downregulated and four other PAP genes were upregulated in the first 2 days of nitrogen starvation (Li et al., 2014).

The reverse reaction, from DAG to phosphatidic acid is catalysed by diacylglycerol kinase (DGK). Both identified DGK genes (Naga_100017g47 and Naga_100007g24) were upregulated. Upregulation of DGK was also found in the nitrogen-starved green microalgae Micractinium pusillum (Y. Li et al., 2012). 
The last step in TAG synthesis is the conversion of DAG into TAG by diacylglycerol acyltransferase (DGAT). This enzyme converts DAG to TAG via an acyl-CoA dependent acylation. There are two types of DGAT genes, DGAT-1 and DGAT-2. One identified DGAT-1 enzyme (Naga_101968g1) was not significant different expressed. On the other hand, six of the identified DGAT-2 genes were significant differential expressed (Naga_100343g3, Naga_100010g31, Naga_100251g8, Naga_100030g42, Naga_100682g2 and Naga100004g173). One of these genes (Naga_100004g173) was annotated as PDAT by Corteggiani Carpinelli et al., 2014 and annotated as DGAT2k by (Alboresi et al., 2016). This gene was downregulated approximately twofold from 0.8 days of nitrogen starvation onwards. In research by Corteggiani Carpinelli et al. 2014, none of the predicted DGAT genes in $N$. gaditana were differentially expressed upon 3 or 6 days of nitrogen starvation (Corteggiani Carpinelli et al., 2014). In Nannochloropsis oceanica, however, seven putative DGAT genes were upregulated and six others were downregulated under nitrogen starvation (Li et al., 2014). For C. reinhardtii two of five DGAT genes were upregulated under nitrogen starvation and were suggested to be involved in TAG synthesis (Boyle et al., 2012). Overexpression of a DGAT-2 from C. reinhardtii in Nannochloropsis showed increased TAG accumulation under phosphorus starvation (Iwai et al., 2015).

In general, different copies of genes with same function involved in acyl-CoA dependent TAG pathway in Nannochloropsis gaditana showed contradictory results with one being upregulated and the other one being downregulated. Indicating that they might be different localized. This complex regulation of the different expression levels for genes with the same functions makes it difficult to draw concrete conclusions regarding regulation of TAG production pathway.

\subsubsection{TAG synthesis via acyl-CoA independent pathway}

Translocation of intact EPA from the polar lipids to TAG takes place under the conditions used in this work as proven in Chapter 2. In order to unravel the cellular mechanisms responsible for translocation, genes involved in acyl-CoA independent pathways were analysed. Enzymes possibly involved in this pathway include phospholipid: diacylglycerol acyltransferase (PDAT) and lipases (Figure 3.6). 


\begin{tabular}{|c|c|c|c|c|c|c|c|c|c|c|}
\hline & to & to.1 & to.3 & to.8 & $\mathbf{t 1 . 8}$ & $\mathrm{t} 2.8$ & $\mathrm{t} 4.8$ & t6.8 & $\mathbf{t} 13.8$ & Accession \\
\hline \multicolumn{11}{|l|}{ PDAT } \\
\hline PDAT (lecithin:cholesterol acyltransferase) & & & & & & & & & & Naga_100065g17 \\
\hline PDAT or DGAT2k (Phospholipid-glycerol acyltransferase) & & & & & & & & & & Naga_100004g173 \\
\hline \multicolumn{11}{|l|}{ Lipases } \\
\hline Acyl transferase/acyl hydrolase/lysophospholipase & & & & & & & & & & Naga_100040g9 \\
\hline Acyl transferase/acyl hydrolase/lysophospholipase & & & & & & & & & & Naga_100012g87 \\
\hline Acyl transferase/acyl hydrolase/lysophospholipase & & & & & & & & & & Naga_100343g4 \\
\hline Acyl transferase/acyl hydrolase/lysophospholipase & & & & & & & & & & Naga_100055g28 \\
\hline Acyl transferase/acyl hydrolase/lysophospholipase & & & & & & & & & & Naga_100156g4 \\
\hline Calcium-independent phospholipase a2-gamm & & & & & & & & & & Naga_100251g1 \\
\hline Calcium-independent phospholipase a2-gamma & & & & & & & & & & Naga_100090g11 \\
\hline Esterase lipase & & & & & & & & & & Naga_100016g85 \\
\hline Esterase lipase thioesterase family protein & & & & & & & & & & Naga_100147g12 \\
\hline Gdsl esterase lipase & & & & & & & & & & Naga_100183g2 \\
\hline GdsI lipase acylhydrolase family protein & & & & & & & & & & Naga_100099g8 \\
\hline Group xv phospholipase a2 & & & & & & & & & & Naga_100053g23 \\
\hline Hormone-sensitive lipase & & & & & & & & & & Naga_100150g1 \\
\hline Lipase & & & & & & & & & & Naga_100241g4 \\
\hline Lipase (putative) & & & & & & & & & & Naga_100889g1 \\
\hline Lipase & & & & & & & & & & Naga_100011g88 \\
\hline Lipase (family) & & & & & & & & & & Naga_100046g19 \\
\hline Lipase (family) & & & & & & & & & & Naga_100016g73 \\
\hline Lipase (family) & & & & & & & & & & Naga_100718g2 \\
\hline Lipase-like & & & & & & & & & & Naga_100530g1 \\
\hline Lipase (domain) & & & & & & & & & & Naga_100012g35 \\
\hline Lipase, class 3 & & & & & & & & & & Naga_100101g8 \\
\hline Lipase, class 3 & & & & & & & & & & Naga_100008g63 \\
\hline Lipase, class 3 & & & & & & & & & & Naga_100171g1 \\
\hline Lipase, class 3 & & & & & & & & & & Naga_100529g6 \\
\hline Lipase, class 3 & & & & & & & & & & Naga_100043g24 \\
\hline Lipase, class 3 & & & & & & & & & & Naga_100104g14 \\
\hline Lipase, class 3 & & & & & & & & & & Naga_100271g1 \\
\hline Lipase, class 3 & & & & & & & & & & Naga_100426g5 \\
\hline TAG lipase & & & & & & & & & & Naga_100057g25 \\
\hline TAG lipase & & & & & & & & & & Naga_100013g62 \\
\hline TAG lipase & & & & & & & & & & Naga_100045g8 \\
\hline TAG lipase- cholesterol esterase & & & & & & & & & & Naga_101607g1 \\
\hline Lysophospholipase & & & & & & & & & & Naga_100017g25 \\
\hline Lysophospholipase 1 & & & & & & & & & & Naga_100436g1 \\
\hline Lysophospholipase-like 1 & & & & & & & & & & Naga_100017g37 \\
\hline Phospholipase A2 & & & & & & & & & & Naga_100247g4 \\
\hline Phospholipase B & & & & & & & & & & Naga_100439g1 \\
\hline Phospholipase d & & & & & & & & & & Naga_100454g3 \\
\hline Phospholipase membrane-associated & & & & & & & & & & Naga_100007g77 \\
\hline sn1-specific diacylglycerol lipase alpha-like protein & & & & & & & & & & Naga_100020g21 \\
\hline & & & & & & & & & & \\
\hline Log2Fold change & $<-3$ & $<-2$ & $<-1$ & $<-0.58$ & 0 & $>0.58$ & $>1$ & $>2$ & $>3$ & \\
\hline
\end{tabular}

Figure 3.6. $\log _{2}$ Fold changes of gene expression levels compared to nitrogen replete conditions over time of the identified genes which might be involved in TAG synthesis via the acyl-CoA independent pathway. PDAT: phospholipid: diacylglycerol acyltransferase. 
In the sequenced genomes one PDAT gene was annotated (Nga02737) (Radakovits et al., 2012) which had the most comparable sequence to Naga_100065g17 which was, however, annotated as lecithin:cholesterol acyltransferase (Corteggiani Carpinelli et al., 2014). This gene was described as putative PDAT by (Dolch et al., 2017) and as PDAT by (Alboresi et al., 2016). Another gene (Naga_100004g173) was annotated by (Corteggiani Carpinelli et al., 2014) as PDAT but (Alboresi et al., 2016) annotated this gene as DGAT2k. The PDAT gene (Naga_100065g17) was upregulated upon nitrogen starvation and increased up to 2.4-fold upon nitrogen starvation from day 2. This was similar to what was found for Nannochloropsis oceanica were PDAT was approximately 50 percent upregulated after two days of nitrogen starvation (Li et al., 2014). In Phaeodactylum tricornutum the expression of PDAT also increased under nitrogen starvation (Mus et al., 2013). This is also in accordance with the measured translocation of EPA from the first day of nitrogen starvation onward (Chapter 2). As PDAT was upregulated from the start of nitrogen starvation, translocation of membrane lipids might be a result of this enzyme. A knock out of PDAT in C. reinhardtii showed a decrease of $25 \%$ of TAG at 2 days of nitrogen starvation, confirmed a role in nitrogen starvation. PDAT only increased under nitrogen starved conditions showing its role during stress conditions (Boyle et al., 2012). PDAT from $C$. reinhardtii was shown to have a broad substrate specificity (Yoon et al., 2012). In Chapter 2, the translocation was more specific for EPA than for other fatty acids. Therefore, it would be interesting to study the substrate specificity of PDAT for EPA. The PDAT or DGAT gene (Naga_10004g173) was downregulated from 1 day of nitrogen starvation onwards up to 2-fold. In contrast to our result, no differential expression of PDAT (Naga_100004g173) in N. gaditana was found upon nitrogen starvation (Corteggiani Carpinelli et al., 2014).

Fatty acids can also be liberated from membrane lipids, like phospholipids and galactolipids, by lipases and used for TAG synthesis. The differentially expressed lipase genes are shown in Figure 3.6.

Several types of lipases identified showed differential expression. Several phospholipases and lysophospholipases were upregulated, suggesting a role during nitrogen starvation. The largest upregulation was shown in phospholipid B (Naga_100439g1) which was had very low counts at the start and was activated upon nitrogen starvation. In P. tricornutum and $N$. oceanica phospholipases were also upregulated during nitrogen starvation (Li et al., 2014; Mus et al., 2013). Other phospholipases were, however, clearly downregulated (e.g. Naga_100454g3 and 
Naga_100247g4). For Chlamydomonas reinhardtii it was shown that a galactoglycerolipid lipase was required for TAG accumulation under nitrogen starvation (X. Li et al., 2012). No specific galactoglycerollipid lipase was annotated in N. gaditana.

Lipase class 3 are lipases targeting TAG. Several lipases class 3 are upregulated upon nitrogen starvation (Naga_100008g63, Naga_100171g1, Naga_100529g6, Naga_100043g24 and Naga_100104g14). Two lipases class 3 were downregulated (Naga_100271g1 and Naga_100426g5). The upregulation suggests increase in TAG degradation, what may decrease TAG production. Therefore, blocking this degradation by inhibiting these lipases might be a strategy to increase TAG production. The classification of lipases with different substrate specificity is however challenging based on sequences (Miller et al., 2010). In C. reinhardtii TAG lipases were also upregulated and it was suggested that these might play a role in releasing fatty acids from membrane lipids for TAG synthesis (Boyle et al., 2012). More research on the specificity of the different lipases is necessary to elucidate the specific enzymes. It would be interesting to test whether one of these enzymes (PDAT or lipases) would have an increased substrate specificity for EPA since this would indicate involvement in the high affinity translocation of EPA as measured in

\section{Chapter 2.}

\subsubsection{Eicosapentaenoic acid (EPA) synthesis}

To study the EPA synthesis during nitrogen starvation, the transcriptomic regulation of several elongases and desaturases involved in the EPA synthesis pathway were measured in time (Figure 3.7). The end products of de novo fatty acids synthesis: palmitic acid, stearic acid or oleic acid produced by the fatty acid synthesis complex, are the substrates for EPA synthesis. 


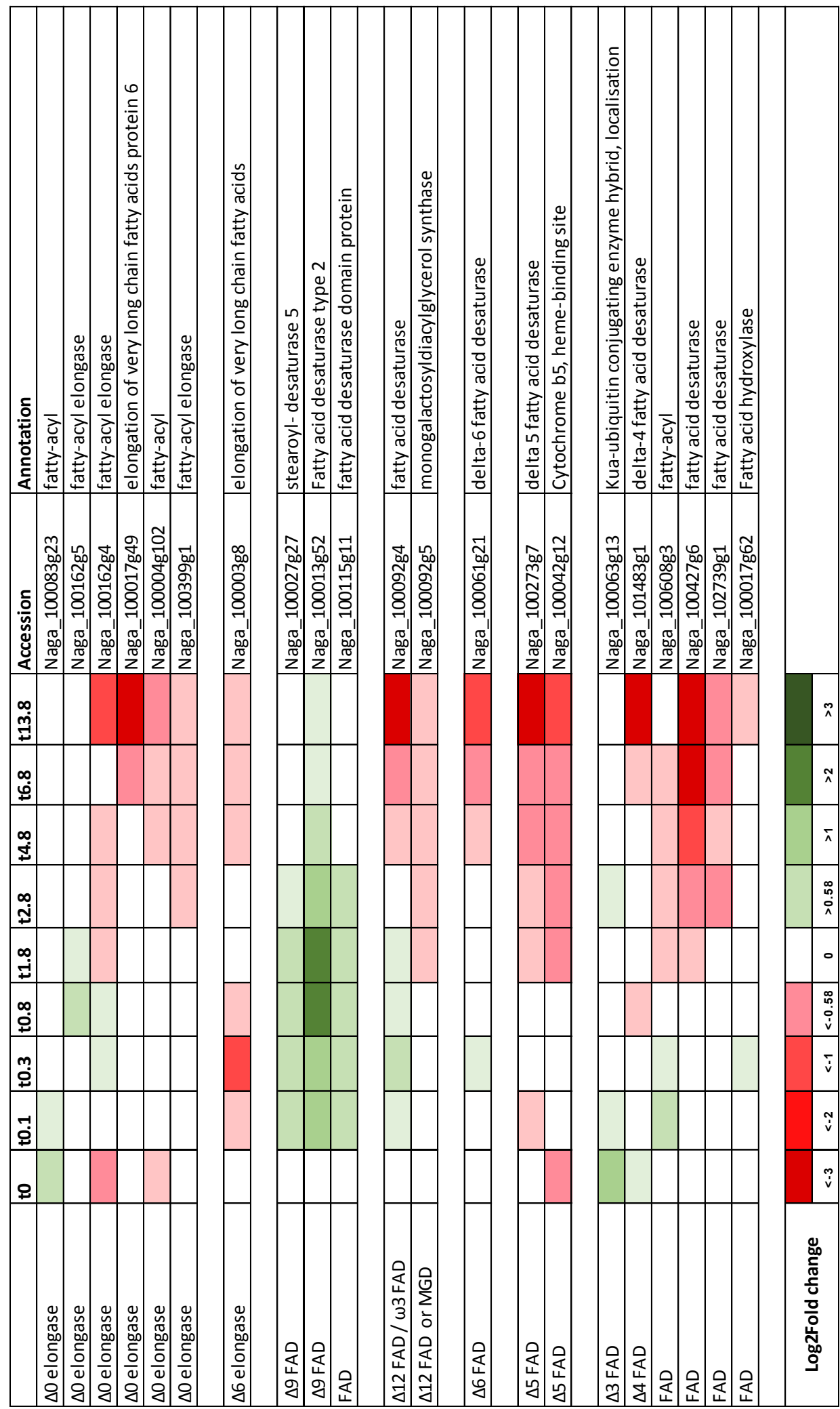

후

동

ㅇํㅇ

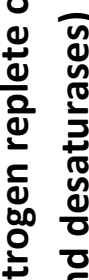

든

$+\check{y}$

인

을 은

产 焉

을

峁吉

$\stackrel{50}{5} .5$

แั๊

兵 ?

응

ถั

옹

ஸे

닌 음

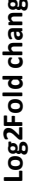

话 
The first step in the EPA synthesis is the $\Delta 0$ elongation of palmitic acid. Several elongases have been identified as $\Delta 0$ elongases (Naga_100083g23, Naga_100162g5, Naga_100162g4, Naga_100004g102, Naga_100017g49 and Naga_100399g1) (Dolch et al., 2017).

The $\Delta 9$-desaturases were the only identified desaturase which was clearly upregulated (Naga_100027g27, Naga_100013g52 and Naga_100115g11) (Ajjawi et al., 2017; Dolch et al., 2017). $\Delta 9$-desaturase was also shown to be upregulated in $N$. oceanica and C. reinhardtii during nitrogen starvation (Li et al., 2014; Miller et al., 2010; Valledor et al., 2014). The increased expression of $\Delta 9$-desaturase correlates with the increase in oleic acid (C18:1). This upregulation was suggested to have a role in prevention of excess reactive oxygen species (Li et al., 2014). The increase in oleic acid upon nitrogen starvation was also reported for different Nannochloropsis species (Rodolfi et al., 2009; Simionato et al., 2013; Xiao et al., 2013). This suggests that EPA synthesis is arrested at oleic acid. Silencing steroyl-ACP desaturase ( $\Delta 9$-desaturase), which converts stearic acid into oleic acid, resulted in an increased stearic acid content in TAG (de Jaeger et al., 2017). The abundance of stearoyl-ACP desaturase proteins was decreased under initial and long term nitrogen depletion in $N$. oceanica (Dong et al., 2013). The increase in oleic acid might indicate that the next desaturation step with $\Delta 12$-desaturase is rate limiting. By overexpression of this gene, linoleic acid was shown to be further converted to PUFAs resulting in increased arachidonic acid in TAG (Kaye et al., 2015). In the present study, the $\Delta 12$-desaturase (Naga_100092g4) was upregulated during the first 2 days of nitrogen starvation and downregulated afterwards. Another identified $\Delta$ 12-desaturase (Naga_100092g5), which was also annotated as monogalactosyldiacylglycerol synthase (MGD) was downregulated from day 2 onwards. In C. reinhardtii and Neochloris oleoabundans $\Delta 12$-desaturase was downregulated during nitrogen starvation (Rismani-Yazdi et al., 2012; Valledor et al., 2014). The same gene responsible for $\Delta 12$ desaturation was suggested to also being responsible for the $\omega 3$ desaturation of arachidonic acid (ARA) to eicosapentaenoic acid (EPA) (Dolch et al., 2017).

The next step in the $\omega 6$ pathway $\Delta 6$-desaturase (Naga_100061g21) was upregulated at 0.3 day and downregulated from day 5 onwards. The $\Delta 6$-desaturase isolated from $N$. oculata was able to desaturate linoleic acid and $\alpha$-linolenic acid, producing $\gamma$-linoleic acid and stearidonic acid, so both $\omega 3$ and 6 precursors could be used (Ma et al., 2011). The following step, $\Delta 6$ elongase (Naga_100003g8) was also downregulated. The identified $\Delta 5$-desaturases (Naga_100273g7 and 
Naga_100042g12) were downregulated up to 4-fold after 14 days of nitrogen starvation. This desaturase was also shown to decrease up to 6.6-fold in C. reinhardtii (Boyle et al., 2012). Overexpression of $\Delta 5$ desaturase was shown to increase PUFA synthesis in $P$. tricornutum (Peng et al., 2014). The last desaturation step uses $\omega 3$ desaturase or $\Delta 17$-desaturase.

Multiple gene copies of the same enzymes indicate possible different localization. Different expression levels make it difficult to identify the exact pathways resulting in TAG accumulation. Moreover, enzymes with multiple functions make it difficult to determine which function they perform in the pathways. Different genes with the same function might operate in different locations. Since a large part (42\%) of the differently expressed genes are annotated as hypothetical or unknown proteins it is possible that important alternative enzymes involved in the studied pathway are unknown. Therefore, improvement of the annotation would help to identify more genes or additional gene copies involved in the studied pathways. Furthermore, more research on the specificity of the enzymes like PDAT or the lipases would improve our understanding of the pathways and substrates involved.

Since transcriptome analysis only gives information about the transcriptional regulation and not on the translation into protein synthesis, proteomic analysis of the discussed pathways would increase our understanding of the active pathways.

Another strategy which could be used to identify key genes in TAG accumulation is comparative genomics. For example Cabanelas et al., 2016, selected a population with increased TAG productivity of Chlorococcum littorale by fluorescence assisted cell sorting (FACS). The difference in expression levels of this population compared to the parental strain might indicate the genes involved in the higher TAG productivity. 


\subsection{CONCLUSIONS}

Transcriptional expression levels of genes involved in TAG and EPA synthesis showed a complex regulation. Multiple copies of the genes were identified might indicate different localization in the cell. Some of these gene copies involved in the acyl-CoA dependent pathway for de novo TAG synthesis were upregulated. The genes which might be involved in the translocation of EPA from membrane lipid to TAG were PDAT and lipases. PDAT gene and multiple lipases were upregulated suggesting a role during nitrogen starvation. Therefore, these enzymes are considered important during nitrogen starvation. The EPA synthesis pathway showed upregulation of $\Delta 9$ fatty acid desaturase what yielded an increase in oleic acid. The other identified desaturases and elongases involved in this pathway were downregulated during nitrogen starvation. Improvements in annotation and enzyme specificity will help understanding the pathways involved in TAG synthesis during nitrogen starvation 


\subsection{SUPPLEMENTS}

\subsubsection{Dry weight and cell concentration comparison}

Comparison of growth results of these experiments with previously obtained data under the same growth conditions (Chapter 2).
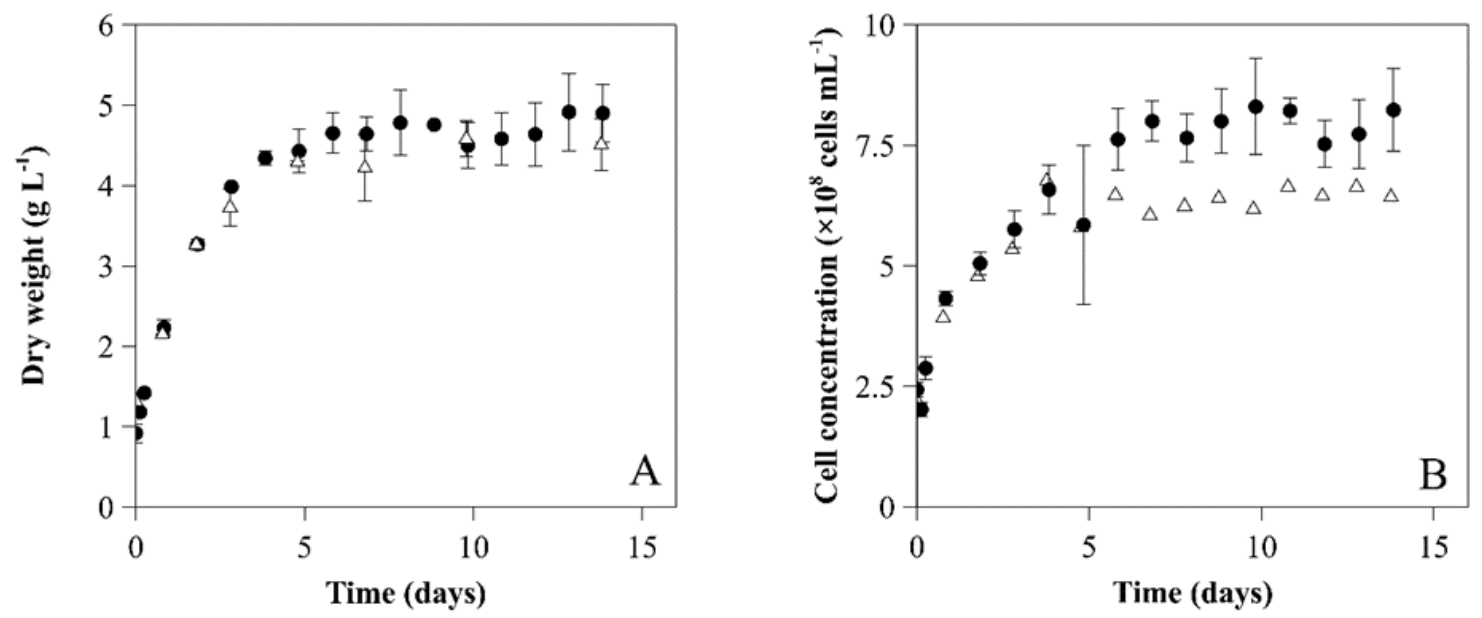

Figure S3.8. Average dry weight concentration $\left(\mathrm{g} \mathrm{L}^{-1}\right)(\mathrm{A})$ and average cell concentration (cells $\mathrm{mL}^{-1}$ ) (B) for this experiment (circles) and the cell concentration of one experiment of previously data Chapter 2 (triangles) from the moment of nitrogen starvation. The error bars show the absolute deviation between two biological photobioreactor experiments $(n=2)$. 


\subsubsection{Fatty acid analysis comparison}

Comparison fatty acid data with previously obtained data under the same growth conditions (Chapter 2).
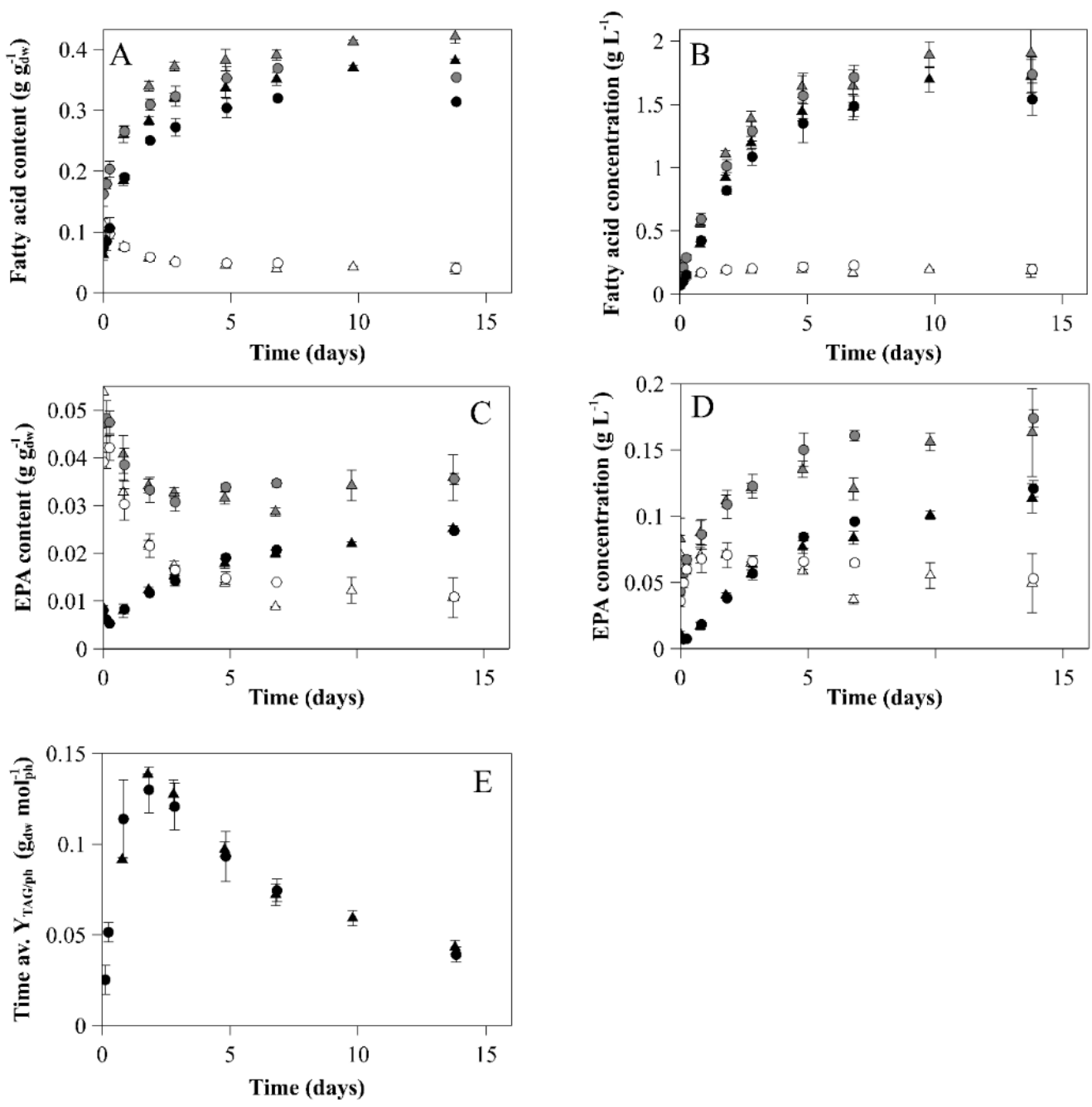

Figure S3.9. Average fatty acid content $\left(\mathrm{g} \mathrm{gdw}^{-1}\right)$ (A) and fatty acid concentration ( $\mathrm{g} \mathrm{L}^{-1}$ ) (B) in TAGs (black), polar lipids (PL) (white) and total lipids (grey, TAG + PL). The average EPA content $\left(g g_{d w}{ }^{-1}\right)(C)$ and EPA concentration ( $\mathrm{g} \mathrm{L}^{-1}$ ) (D) in TAG (black), polar lipids (white) and total lipids (grey, TAG + PL). The average time-averaged TAG yield on light (gTAG molph ${ }^{-1}$ ) including correction for energy necessary for inoculum production (E). In all graphs the circles show the results of these experiments and the triangles show the result of previous results (Chapter 2). All from the start of nitrogen starvation. The error bars show the absolute deviation between two biological photobioreactor experiments $(n=2)$. 


\subsubsection{PCA plot}

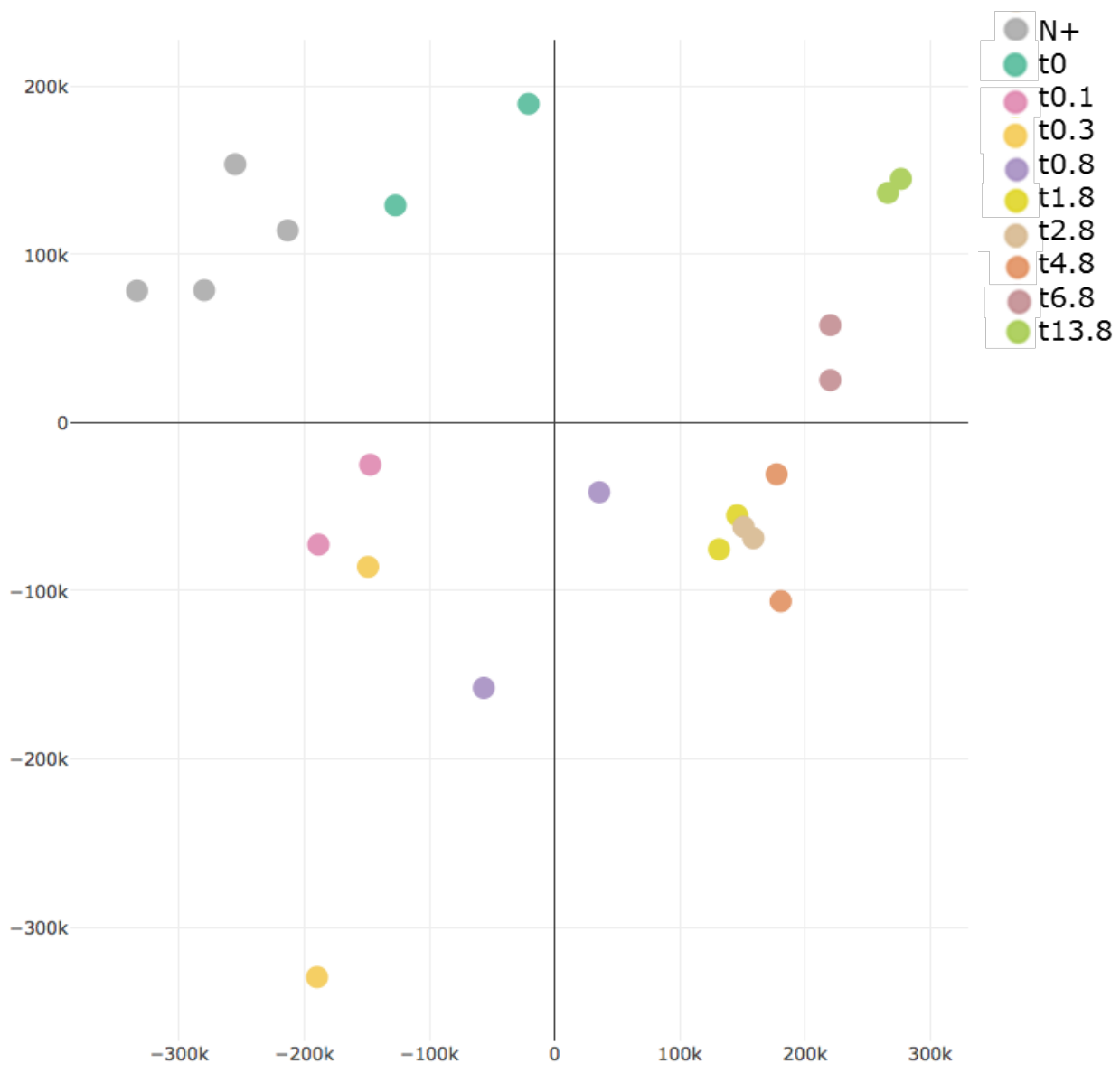

Figure S3.10. Principle component analysis (PCA) of all RNA expression data from all analysed samples to show reproducibility. The plot indicates good reproducibility between biological replicates, except for t0.3. 


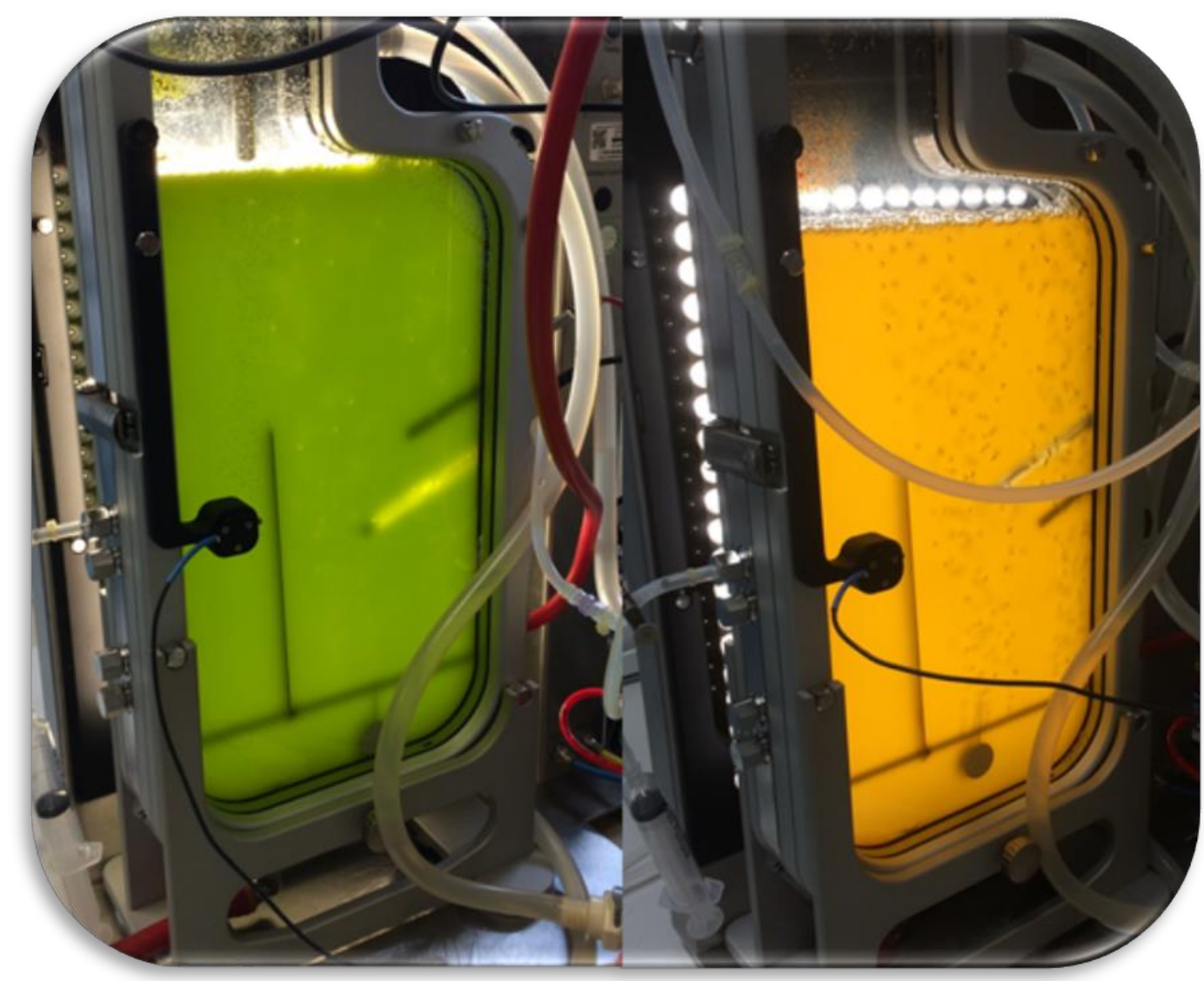

Photobioreactor with growing (left) and nitrogen starved (right) microalgae. 


\section{Chapter 4}

\section{Effect of nitrogen addition on lipid productivity of nitrogen starved Nannochloropsis gaditana}

This chapter is accepted for publication in Algal Research as:

JH Janssen, J Kastenhofer, JA de Hoop, PP Lamers, RH Wijffels, MJ Barbosa.

Effect of nitrogen addition on lipid productivity of nitrogen starved Nannochloropsis gaditana 


\section{ABSTRACT}

Microalgae are a sustainable source of lipids. A commonly used strategy for lipid accumulation in microalgae is a two-step batch cultivation, with a growth phase followed by a nitrogen starvation phase. A problem with this process is the decrease in photosynthetic efficiency during the nitrogen starvation phase, which leads to low lipid productivities. In this research, a new process strategy was studied with the aim to improve lipid productivity of the microalgae Nannochloropsis gaditana. The nitrogen concentrations were chosen to assure consumption of most part of the nitrogen during the night. An improvement of the photosystem II maximum quantum yield and an increase in the dry weight and TAG concentration was achieved from day 7 of nitrogen starvation onwards when the culture was fed with nitrogen each night compared to a culture without nitrogen addition. Consequently, the time-average TAG yield on light was also higher after 7 days of nitrogen starvation. However, since the maximal time-averaged triacylglycerol (TAG) yield on light was reached after 3 days of nitrogen starvation, the improved photosynthetic activity did not lead to an increase of the maximal time-averaged TAG yield on light. The culture with nitrogen addition had a higher protein concentration (1.1 compared to $\left.0.7 \mathrm{~g} \mathrm{~L}^{-1}\right)$, showing that the added nitrogen was mainly used for protein production. A higher chlorophyll a content (2.0 compared to $0.8 \mu \mathrm{g} \mathrm{mg}^{-1}$ ) showed improved photosystem and that a small part of nitrogen was used for chlorophyll a. Small nightly nitrogen additions during batch cultivation of nitrogen starved $N$. gaditana did result in improvement in photosystem II maximal quantum yield, biomass concentration, TAG production and a higher time-averaged maximal TAG yield on light, after 7 days of nitrogen starvation. 


\subsection{INTRODUCTION}

Microalgae are a sustainable source of lipids, proteins and carbohydrates for food, feed and fuel applications (Wijffels and Barbosa, 2010). Higher lipid productivities, however, are required to achieve an economically feasible process (Ruiz et al., 2016). The microalgae Nannochloropsis gaditana is used as a model species because of its ability to accumulate large amount of lipids (Simionato et al., 2013), the available genome sequence and annotation (Corteggiani Carpinelli et al., 2014; Radakovits et al., 2012) and being a robust species shown by already being cultivated outdoors (San Pedro et al., 2016). The lipids in Nannochloropsis are present in a polar lipid (PL) fraction and triacylglycerol (TAG) fraction. One of the often applied strategies to induce TAG accumulation is nitrogen starvation (Breuer et al., 2012; Simionato et al., 2013). TAG accumulation is often applied as a two phase batch system, consisting of a growth phase followed by a nitrogen starvation phase, where TAG accumulates. A problem faced during nitrogen starvation is the decrease in photosynthetic efficiency and biomass productivity leading to low TAG productivities (Benvenuti et al., 2016b; Breuer et al., 2012).

Different process strategies have been investigated to improve photosynthesis during nitrogen starvation. One of the cultivation strategies previously applied to improve TAG productivity was nitrogen limitation in Neochloris oleoabundans and Acutodesmus obliquus. In this strategy limited amount of nitrogen was fed to a continuous culture, to sustain cell growth and at the same time induce TAG accumulation (Klok et al., 2013; Remmers et al., 2017a). This strategy, however, did not increase TAG yield on light compared to a conventional batch cultivation followed by nitrogen starvation. Repeated batch cultivations has also been tested for TAG production. During this strategy part of the nitrogen starved culture is harvested and replaced by fresh cultivation medium containing nitrogen. Due to cell growth the nitrogen is depleted, TAG accumulates and at a certain point in starvation, part of the culture is harvested again and replaced by fresh medium containing nitrogen. For Nannochloropsis sp. it was shown that batch cultivation followed by nitrogen starvation led to higher lipid productivities compared to repeated batch cultivations (Benvenuti et al., 2016b). It was hypothesized that the TAG yield on light was not improved in repeated batch because light energy was not used only for TAG accumulation but also for cell regrowth, after replenishing the nitrogen starved culture with fresh medium. Previous research also showed that 
nitrogen starved cultures of the microalgae species Nannochloropsis oceanica, Chromochloris zofingiensis and Chlamydomonas reinhardtii can increase their photosynthetic efficiency again after nitrogen resupply (Dong et al., 2013; Mulders et al., 2014b; Siaut et al., 2011; Valledor et al., 2014). After nitrogen replenishment, TAG productivity decreases drastically because TAG is used as energy source for the production of biomass and possibly for nitrogen uptake. TAG has been reported to be degraded within hours after nitrogen resupply (Siaut et al., 2011; Valledor et al., 2014). When nitrogen was resupplied to a dark culture of Chlamydomonas reinhardtii, after three days of nitrogen starvation, it first induced starch degradation and later TAG degradation to support cell growth (Siaut et al., 2011). For a mixed culture it was shown that in a repeated batch cultivation the storage compound starch, was maximized when ammonium was supplied as nitrogen source at the start of the dark period rather than in the light period (Mooij et al., 2015).

In this research a new cultivation strategy was tested to improve the TAG yield on light by adding small amounts of nitrogen during each night to a nitrogen starved culture. The nitrogen concentrations were chosen to assure consumption of most part of the nitrogen during the night. We hypothesized that nitrogen addition during the night reduces light loss during the day by maintaining photosynthetic efficiency. In this way, the energy available in the light period can be more efficiently used for TAG accumulation and lead to an increased TAG yield on light (Benvenuti et al., 2016b; Mulders et al., 2014b). During nitrogen starvation microalgal cells degrade their photosystems. By addition of nitrogen, the cells are not nitrogen starved anymore and can direct their energy again to photosynthesis. The added nitrogen can be used for de novo protein synthesis and maintain or rebuild the photosystems. Energy necessary to assimilate nitrogen during the night could be provided by for example carbohydrate storage. A more efficient photosystem can more efficiently produce TAG and thus improve TAG yield on light. 


\subsection{MATERIALS AND METHODS}

\subsubsection{Strains, pre-cultivation conditions and cultivation medium}

The marine microalgae Nannochloropsis gaditana CCFM-01 was obtained from the Microalgae Collection of Fitoplancton Marino S.L.. Pre-cultures were maintained in $250 \mathrm{~mL}$ Erlenmeyer flasks containing $100 \mathrm{~mL}$ filter sterilized (pore size $0.2 \mu \mathrm{m}$ ) cultivation medium and incubated in an orbital shaker incubator (100 rpm), at $25^{\circ} \mathrm{C}$, with air in headspace and an incident light intensity of $30-40 \mu \mathrm{mol} \mathrm{m}^{-2} \mathrm{~s}^{-1}$ with 18:6 hours light dark cycle. The culture media was based on Breuer et al., 2012 and contained: $\mathrm{NaCl} 445 \mathrm{mM}$; $\mathrm{KNO}_{3} 33.6 \mathrm{mM}$; $\mathrm{Na}_{2} \mathrm{SO}_{4} 3.5 \mathrm{mM}$; $\mathrm{MgSO}_{4} \cdot 7 \mathrm{H}_{2} \mathrm{O} 3 \mathrm{mM}$; $\mathrm{CaCl}_{2} \cdot 2 \mathrm{H}_{2} \mathrm{O} 2.5 \mathrm{mM}$; $\mathrm{K}_{2} \mathrm{HPO}_{4} 2.5 \mathrm{mM}$; NaFeEDTA $28 \mu \mathrm{M}$; Na $\mathrm{EDTTA}_{2} \mathrm{H}_{2} \mathrm{O} 80 \mu \mathrm{M}$; $\mathrm{MnCl}_{2} \cdot 4 \mathrm{H}_{2} \mathrm{O} 19 \mu \mathrm{M} ; \mathrm{ZnSO}_{4} \cdot 7 \mathrm{H}_{2} \mathrm{O} 4 \mu \mathrm{M}$; $\mathrm{CoCl}_{2} \cdot 6 \mathrm{H}_{2} \mathrm{O} 1.2 \mu \mathrm{M} ; \mathrm{CuSO}_{4} \cdot 5 \mathrm{H}_{2} \mathrm{O} 1.3 \mu \mathrm{M}$; $\mathrm{Na}_{2} \mathrm{MoO}_{4} \cdot 2 \mathrm{H}_{2} \mathrm{O} 0.1 \mu \mathrm{M}$; Biotin $0.1 \mu \mathrm{M}$; vitamin B1 3.3 $\mu \mathrm{M}$; vitamin $\mathrm{B} 120.1 \mu \mathrm{M}$; and $10 \mathrm{mM} \mathrm{NaHCO}_{3}$. The $\mathrm{pH}$ was adjusted with $\mathrm{NaOH}$ to 7.5. During pre-cultivation in Erlenmeyer flasks 100 mM 4-(2-hydroxyethyl)piperazin-1-ethanesulfonic acid (HEPES) was added as $\mathrm{pH}$ buffer. Prior to inoculation into the photobioreactor the cultures were transferred to $100 \mu \mathrm{mol} \mathrm{m}^{-2} \mathrm{~s}^{-1}$ continuous light with $2.5 \% \mathrm{CO}_{2}$ enriched air. During cultivation in the photobioreactor $\mathrm{KNO}_{3}$ was reduced to $7.02 \mathrm{mM}$, which is sufficient to reach a biomass concentration of $1.4 \mathrm{~g} \mathrm{~L}^{-1}$.

\subsubsection{Experimental and reactor setup}

$N$. gaditana was cultivated in heat-sterilized flat panel photobioreactor with a light path of $20.7 \mathrm{~mm}$ and a working volume of $1.8 \mathrm{~L}$ (Labfors $5 \mathrm{Lux}$, Infors HT, Switzerland, 2010). The reactor was kept at $26{ }^{\circ} \mathrm{C}$ by a waterjacket. The $\mathrm{pH}$ was controlled at 7.5 by on demand addition of sulphuric acid $(2.5 \% \mathrm{v} / \mathrm{v})$ and at the start of the culture sodium hydroxide $(1 \mathrm{M})$. The culture was mixed by filter sterilized air with $2 \% \mathrm{CO}_{2}$ with a flow rate of $1 \mathrm{~L} \mathrm{~min}-1$. The off gas was cooled in a condenser. The light was provided by 260 LED lamps with warm white spectrum (450-620 nm). The incident light intensity was measured with a LI-COR 190-SA $2 \pi$ PAR $(400-700 \mathrm{~nm})$ quantum sensor (LI-COR, USA). The incident light intensity started at $63 \mu \mathrm{mol} \mathrm{m} \mathrm{m}^{-2} \mathrm{~s}^{-1}$ and increased daily, by keeping the outgoing light intensity $30-40 \mu \mathrm{mol} \mathrm{m}^{-2} \mathrm{~s}^{-1}$, up to $636 \mu \mathrm{mol} \mathrm{m} \mathrm{m}^{-2} \mathrm{~s}^{-1}$ and kept constant during the nitrogen starvation period. A day : night cycle of 16 : 8 hours was used. The backside of the reactor was covered with a black cover to prevent interference of environmental light. The microalgae were cultivated in batch mode with two phases; growth phase and nitrogen starvation 
phase when all nitrogen was consumed. The growth phase was inoculated at biomass concentration of $0.1 \mathrm{~g} \mathrm{~L}^{-1}$. Nitrogen runout at a biomass concentration of $1.8 \mathrm{~g} \mathrm{~L}^{-1}$ concentration and this time was considered the start of nitrogen starvation. From this moment on nitrogen was added during the night. Nitrogen addition was performed by adding a concentrated $\mathrm{KNO}_{3}$ solution $(0.29 \mathrm{M})$ at the start of each night (the total volume added was 17 and $23 \mathrm{~mL}$ for experiment 1 and 2). The amount of added nitrogen was tuned to be mostly consumed at the end of the night. Previously experiments showed that the nitrogen uptake rate per amount of biomass decreased with increasing time of nitrogen starvation. This knowledge was used to determine the amount of nitrogen added. The amounts nitrogen added was decreased over the course of the experiment (Table S4.1). The experiment was performed in duplicate photobioreactors. The control experiment had an identical setup and process conditions but without nitrogen addition in the night. Control experiments were also performed in duplicate.

\subsubsection{Offline measurement culture}

The optical density (OD) was measured daily in duplicate at 750, 680 and $480 \mathrm{~nm}$ using a UV-VIS spectrophotometer (Hack Lange DR-6000, light path $10 \mathrm{~mm}$ ). The OD680 and OD480 were used as an estimate for, respectively, the chlorophyll and carotenoid content. To correct for scattering, the OD750 was subtracted and the result was divided by the OD750. From these samples the cell number, cell size, quantum yield and the dry weight-specific optical cross section were also measured. The cell number and size were measured with the Multisizer III (Beckman Coulter) using $50 \mu \mathrm{m}$ aperture tube and after diluting the sample 100 times in ISOTON II diluent. The photosystem II (PSII) maximum quantum yield $\left(F_{v} / F_{m}\right)$ was measured after 15 minutes dark adaptation at room temperature (AquaPen-C 100, PSI, Czech Republic; excitation light $455 \mathrm{~nm}$, saturating light pulse: $\left.3000 \mu \mathrm{mol} \mathrm{m}{ }^{-2} \mathrm{~s}^{-1}\right) . \mathrm{Fv}_{\mathrm{v}} / \mathrm{Fm}_{\mathrm{m}}$ ratio was calculated according to Benvenuti et al., 2015.

The average dry weight-specific optical cross section $\left(\alpha_{c}\right.$ in $\left.\mathrm{m}^{2} \mathrm{~g}^{-1}\right)$ was measured and calculated according to de Mooij et al., 2015 using the absorbance from 400 to $750 \mathrm{~nm}$ with a steps size of $1 \mathrm{~nm}$.

Biomass concentration $\left(C_{x}\right.$ in $\mathrm{g} \mathrm{L}^{-1}$ ) was measured from the moment of nitrogen starvation by measuring the dry weight of the culture. The dry weight was measured 
in triplicate as described by Kliphuis et al., 2012 with the exception that $0.5 \mathrm{M}$ ammonium formate was used to wash the filters.

Regularly, biomass samples were taken, at the start and end of the dark period, to determine the biomass composition. The biomass samples were centrifuged 5 minutes at $4255 \mathrm{~g}$ (Beckman coulter Allegra X-30R centrifuge) and washed twice with $0.5 \mathrm{M}$ ammonium formate, stored at $-20{ }^{\circ} \mathrm{C}$ and lyophilized. Since, the supernatant was reddish turbid and washed away for biomass composition analyses the measured dry weight was corrected for the measured dry weight of the supernatant after centrifugation on a filter. Samples for nitrate analysis were centrifuged at $20238 \mathrm{~g}$ (Eppendorf centrifuge 5424) for 2 minutes and stored at $-20^{\circ} \mathrm{C}$.

The residual nitrate in the supernatant was measured using AQ2 nutrient analyser (Seal Analytical) as described by Benvenuti et al., 2016a. Nitrate was measured after addition at start of the night and at the start of the light period. The dry weight specific nitrogen addition was calculated by dividing the nitrogen concentration by the dry weight concentration. This measurement is only available for one of the duplicate experiments.

Due to limited amount of biomass for sampling in one of the reactors, the dry weight concentration and biomass composition data from day 8 to 13, 15 and 16 were measurements from only one of the reactors. The optical density at $750 \mathrm{~nm}$ and photosystem II quantum yield were measured daily for both duplicate photobioreactors and showed reproducible results. Therefore, it is likely that the duplicate reactors were comparable.

\subsubsection{Biomass composition analyses}

The triacylglycerol (TAG), polar lipid content and fatty acid composition were analysed according to Breuer et al., 2012; León-Saiki et al., 2017. Lipids were extracted from 6-7 mg lyophilized biomass with chloroform: methanol (1:1.25 v/v) mixture with $170 \mu \mathrm{g} \mathrm{mL}-1$ tripentadecanoin (T4257, Sigma Aldrich) and $170 \mu \mathrm{g} \mathrm{mL}^{-1}$ 1,2-dipentadecanoyl-sn-glycero-3-[phospho-rac-(1-glycerol)] (sodium salt) (840446P, Avanti Polar Lipids Inc.) as internal standard for respectively the TAG and polar lipid fraction. The TAG lipids were separated from the polar lipids by different elution on SPE silica gel cartridge (Sep-Pak Vac 6cc, Waters). The separated lipid fractions were methylated and the fatty acid methyl esters (FAMEs) were quantified 
using gas chromatography (GC-FID). The TAG and polar lipid content was calculated by the sum of all fatty acid measured per amount of biomass in that fraction.

The protein content was analysed in triplicate according to Postma et al., 2015. The freeze dried biomass was bead-beated, $3 \times 60 \mathrm{~s}$ at $6500 \mathrm{rpm}$ with $120 \mathrm{~s}$ pause, with lysing buffer. The soluble proteins in supernatant were analysed with the DC protein assay kid (Bio-Rad, U.S.) using bovine serum albumin (Sigma-Aldrich) as protein standard.

The carbohydrate analysis was performed in triplicate on freeze dried biomass which was hydrolysed with $\mathrm{HCl}(2.5 \mathrm{M})$ for $3 \mathrm{~h}$ at $100{ }^{\circ} \mathrm{C}$. After neutralizing with $\mathrm{NaOH}(2.5$ $\mathrm{M})$ and diluting the sample, monosaccharides were quantified colorimetric according to Dubois et al., 1956, with the exception: that the tubes containing the colour reaction were incubated at $35{ }^{\circ} \mathrm{C}$ for 30 minutes and the absorbance was measured at $483 \mathrm{~nm}$, using D-glucose as standard.

Chlorophyll a was extracted from the lyophilized biomass according to Lamers et al., 2010 with methanol : chloroform $(2.5: 2, \mathrm{v}: \mathrm{v})$ with BHT $(0.1 \% \mathrm{w}: \mathrm{v})$ by beat beating for 8 times 60s with $120 \mathrm{~s}$ pause at $2500 \mathrm{rpm}$. After mixing, sonication and washing with Tris : $\mathrm{NaCl}(50 \mathrm{mM}: 1 \mathrm{M}, \mathrm{pH}$ 7.5) the solvent was evaporated and the sample was dissolved in methanol. Chlorophyll $a$ was separated and quantified using reversed-phase high-performance liquid chromatography (RP-HPLC) with a UVVIS detector (660 nm). HPLC analysis was performed in a Shimadzu system coupled with a photo-diode array detector (SPD-M20A) and an Acclaim C30 LC reversedphase column (Thermo Scientific) with a column temperature of $30^{\circ} \mathrm{C}$ and flow rate of $1.5 \mathrm{~mL} \mathrm{~min} \mathrm{~m}^{-1}$. Three mobile phases were used: A) acetonitrile B) methanol : ethyl acetate (1:1 v:v) C) acetic acid in water (200 mM). Gradient method (minutes - \%A $\% \mathrm{~B}-\% \mathrm{C}): 0-85-14.5-0.5 / 2-85-14.5-0.5 / 15-65-34.5-0.5 / 25-65-34.5$ -0.5 and $30-85-14.5-0.5$.

\subsubsection{Calculation TAG yield}

The time-averaged TAG yield on light $\left(\mathrm{Y}_{\mathrm{TAG} / \mathrm{ph}}\right.$ in $\left.\mathrm{g}_{\mathrm{TAG}} \mathrm{mol}_{\mathrm{ph}}{ }^{-1}\right)$ was calculated according to Remmers et al., 2017a. The TAG concentration produced from start of nitrogen starvation was divided by the volumetric photon supply rate $\left(r_{\mathrm{ph}}\right.$ in $\left.\mathrm{mol}_{\mathrm{ph}} \mathrm{L}^{-1}\right)$ from the start of nitrogen starvation. The volumetric photon supply rate included the light necessary for inoculum, calculated from the biomass concentration at the start of 
nitrogen starvation $\left(C_{x}\right.$ in $\left.g^{-1}\right)$ and assuming a biomass yield on light $\left(Y_{x / p h}\right)$ of $1 \mathrm{~g}_{\mathrm{x}} \mathrm{mol}_{\mathrm{ph}}{ }^{-1}$.

\subsection{RESULTS AND DISCUSSION}

In this research, Nannochloropsis gaditana was subjected to nitrogen starvation in a batch process with a 16:8 hours day:night cycle, in which nitrogen was added at the start of each night. Results were compared to a control experiment without nitrogen addition and are shown from the start of nitrogen starvation (time 0 ). The results show that almost all (83\%) nightly added nitrogen was consumed during the night (Figure 2) and the remainder during first hours of the day. Nightly nitrogen addition led to higher biomass production from day 7 of nitrogen starvation onwards. This was reflected by a higher biomass concentration (Figure $1 \mathrm{~A}$ ), a higher cell number (Figure $1 \mathrm{~B}$ ) and a higher PSII maximum quantum yield (Figure $3 \mathrm{~A}$ ). This extra biomass consisted of additional protein (main sink for the added nitrogen, Figure 6), additional chlorophyll $a$ (minor sink for the added nitrogen, Figure $3 \mathrm{~B}$ ) and most importantly additional TAG (Figure 5A). The composition of this additional biomass remained constant from day 8 onwards (Figure 4). As a result of the increased TAG production from day 8 onwards, the TAG yield on light also increased in that period (Figure 5B). 


\subsubsection{Biomass concentration}
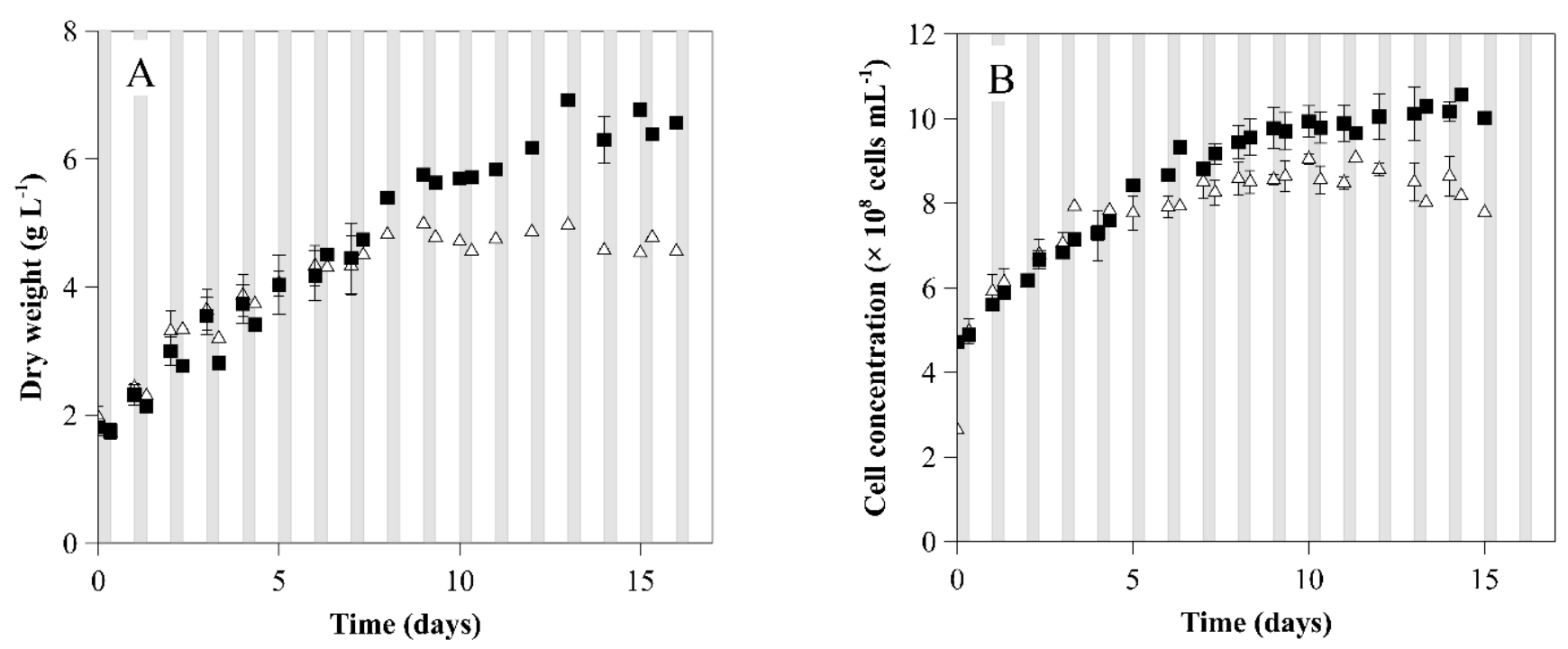

Figure 4.1. Average dry weight concentration $\left(\mathrm{g} \mathrm{L}^{-1}\right)(\mathrm{A})$ and average cell concentration $\left(\times 10^{8}\right.$ cells $\left.\mathrm{mL}^{-1}\right)(B)$ from the moment of nitrogen starvation for culture with nitrogen addition (squares) and without nitrogen addition (triangles). The grey bars represent the night periods. Error bars show absolute deviation between the biological duplicate experiments. The dry weight measurements on day 8 to 13,15 and 16 were measurement from one of the reactors. The cell number measurements were from one reactor when no error bars are shown.

In general, during nitrogen starvation the biomass concentration increased due to lipid accumulation. The nitrogen starvation was started at similar dry weight concentration for the cultures with and without nitrogen addition (1.81 \pm 0.13 and $1.96 \pm 0.17 \mathrm{~g} \mathrm{~L}^{-1}$ ) (Figure 4.1A). The dry weight concentration increased similarly for both cultures during the first 6 days. From day 7 onwards the dry weight of the culture with nitrogen addition kept increasing, while a constant biomass concentration was observed for the control (Figure 1A). The maximum dry weight concentrations reached were 6.9 and $4.8 \mathrm{~g} \mathrm{~L}^{-1}$ for the culture with and without nitrogen addition, respectively. The same trend was observed for cell concentration (Figure 4.1B). The cell number approximately doubled from the start of the nitrogen starvation until day 7 , meaning that the cells on average still divided once after the start of nitrogen starvation. From day 6 only the cell concentration of the culture with nitrogen additions still increased and was thus dividing. 
Nannochloropsis oculata also showed cell division after nitrogen starvation and division of nitrogen over the daughter cells (Flynn et al., 1993). No large differences in cell size were found between both cultures (data not shown). In the four initial nights (except for the control of day 2 ) the dry weight concentration decreased during the night, providing energy during the night period. No difference in this decrease between the culture with and without nitrogen addition was found indicating that the energy needed for nitrogen uptake was probably low compared to other energy requirements of the cell during the night. From day 5 onwards there was a less clear decrease in the dry weight concentration during the nights (Figure 4.1A).

\subsubsection{Nitrogen addition and consumption}

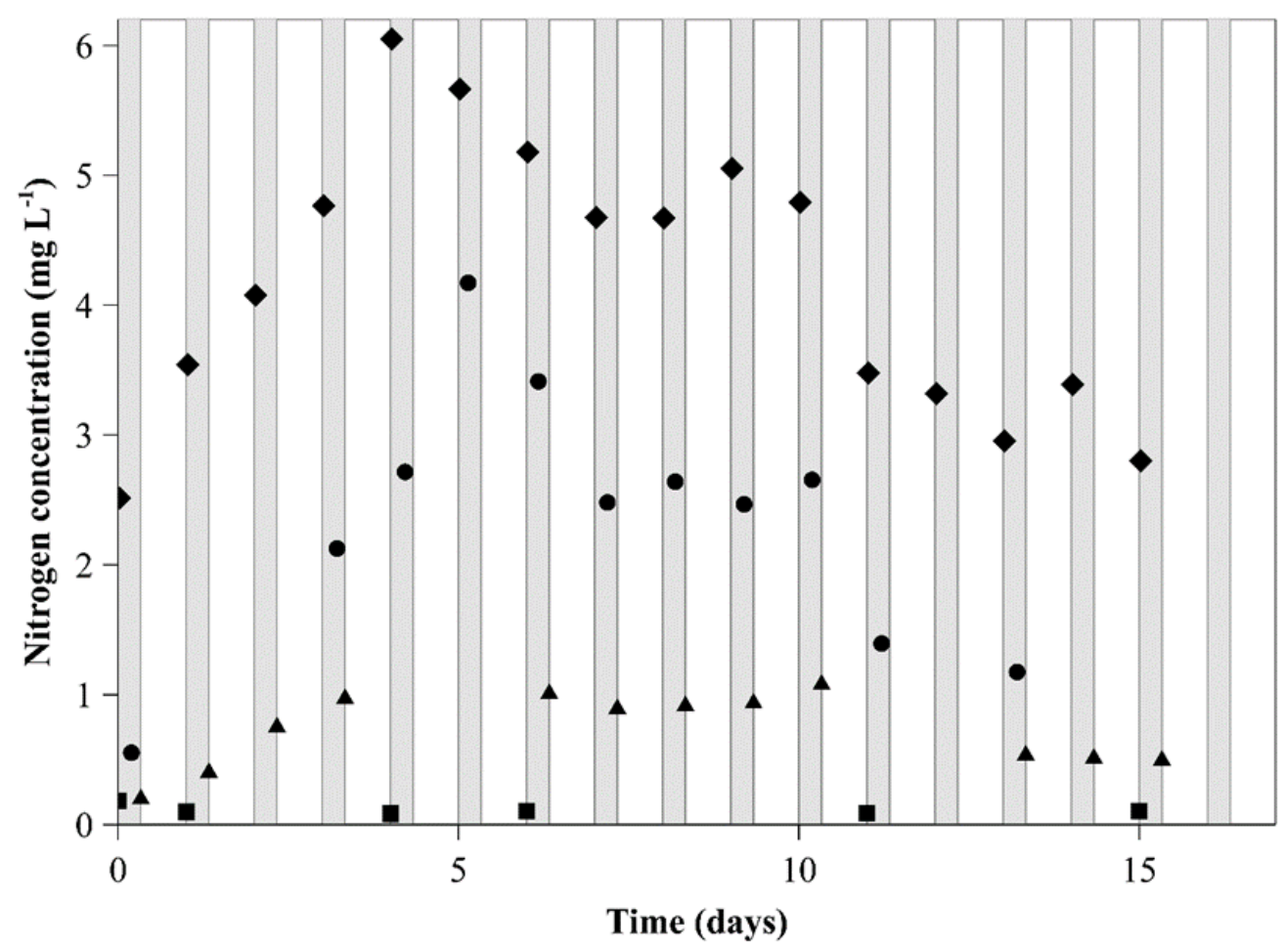

Figure 4.2. Nitrogen concentration $\left(\mathrm{mg} \mathrm{L}^{-1}\right)$ in supernatant after nitrogen addition (diamonds), during night (circles), at the end of the night (triangles) and at the end of the day (squares). The grey bars represent the night period. Graph shows the measurements for one of the duplicate experiments. 
Nitrogen was added daily at the start of the night. The amount of nitrogen added per dry weight was decreased over time to facilitate complete take up of nitrogen during the night (Figure 4.2, diamonds). On average, $83 \pm 4 \%$ of the added nitrogen was consumed during the night and about $0.7 \pm 0.3 \mathrm{mg} \mathrm{L}^{-1}$ nitrogen $(0.2 \mathrm{mg}$ nitrogen per gram dry weight) was left in the culture at the end of the night (Figure 4.2, triangles). It could be because the nitrate uptake rate was dependent on the nitrate concentration. For Chlorella vulgaris it was shown that the nitrate uptake rate increased with nitrate concentration in the medium (Jeanfils et al., 1993). Nitrate induces expression of genes responsible for nitrogen assimilation (Sanz-Luque et al., 2015). Another possibility could be that the time exposed to nutrient starvation decreased the nitrogen uptake rate. The nitrogen left at the end of the night was consumed during the day (Figure 4.2, squares). The total amount of nitrogen added was 70 and $96 \mathrm{mg}$ nitrogen in respectively, 14 and 16 nights, for the two duplicate experiments. Different amounts were added to keep the amount of nitrogen added per dry weight as similar as possible and due to the difference in length of the experiment.

\subsubsection{Photosynthetic capacity and pigmentation}
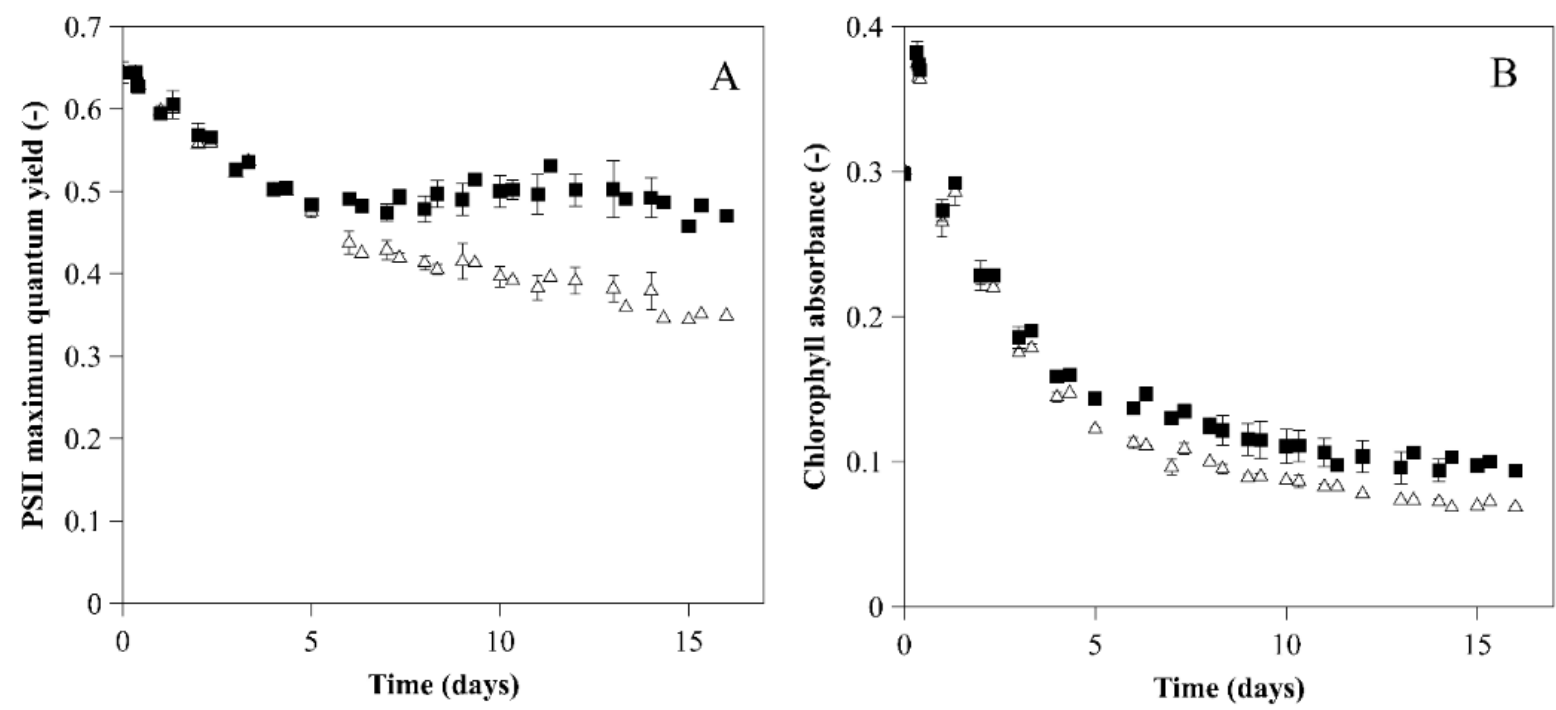

Figure 4.3. Average PSII maximum quantum yield $(A)$ and chlorophyll absorbance at $680 \mathrm{~nm}$ corrected for scattering (B) for the cultures with (squares) and without nitrogen addition (triangles). The error bars show the absolute deviation between the averages of the two duplicate experiments. 
The PSII maximum quantum yield rapidly decreased after nitrogen starvation, which is attributed to the diminished activity or amount of photosystem II (Simionato et al., 2013). This decrease of the PSII maximum quantum yield indicates a decrease photosynthetic activity. From day 6 the PSII maximum quantum yield stabilized at 0.5 for the culture with nitrogen addition while the culture without nitrogen addition decreased further to 0.35 (Figure 4.3). This indicates that the addition of nitrogen during the night led to an improvement of photosystem II. The average dry weightspecific optical cross section and the carotenoid content, based on absorbance 480 $\mathrm{nm}$ and corrected for scattering, showed no difference for the nitrogen added culture and the culture without addition (data not shown). On the contrary, the chlorophyll absorbance at $680 \mathrm{~nm}$ corrected for scattering showed a small increase for the culture with nitrogen addition. This difference in chlorophyll content was confirmed by HPLC analysis of chlorophyll $a$ for one of the duplicate experiment. The chlorophyll $a$ content before nitrogen starvation was $21 \mu \mathrm{g} \mathrm{mg}^{-1}$. This decreased to 2.0 and $0.8 \mu \mathrm{g} \mathrm{mg}^{-1}$ at day 16 for the cultures with and without nightly nitrogen addition, respectively. The chlorophyll $a$ content of the culture with nitrogen addition was 2.5 times higher compared to the control without nitrogen addition, suggesting improved photosystem pigmentation. Other research has shown that nitrogen addition to nitrogen starved cultures could change their physiology back to pre-starvation phase, including an increase in pigments and proteins. It can, however, not be excluded that the difference in chlorophyll $a$ content was caused by photoacclimation (Falkowski and Owens, 1980) due to the difference in biomass concentration of the cultures at the end of the nitrogen starvation phase. The same incident light intensity with different biomass concentration leads to a different light gradient in the photobioreactor. The total amount of chlorophyll a present at day 16 in the reactor was approximately $15.6 \mu \mathrm{g}$ in the nitrogen added culture compared to $4.4 \mu \mathrm{g}$ for the control culture. Based on the molecular formula of chlorophyll $a$, the nitrogen content is $6.27 \% \mathrm{w} / \mathrm{w}$. Therefore, $0.7 \mu \mathrm{g}$ nitrogen was necessary for the extra chlorophyll $a$ in the nitrogen added culture. This is a very small part of the total amount of nitrogen added during the nights. 


\subsubsection{Biomass composition}

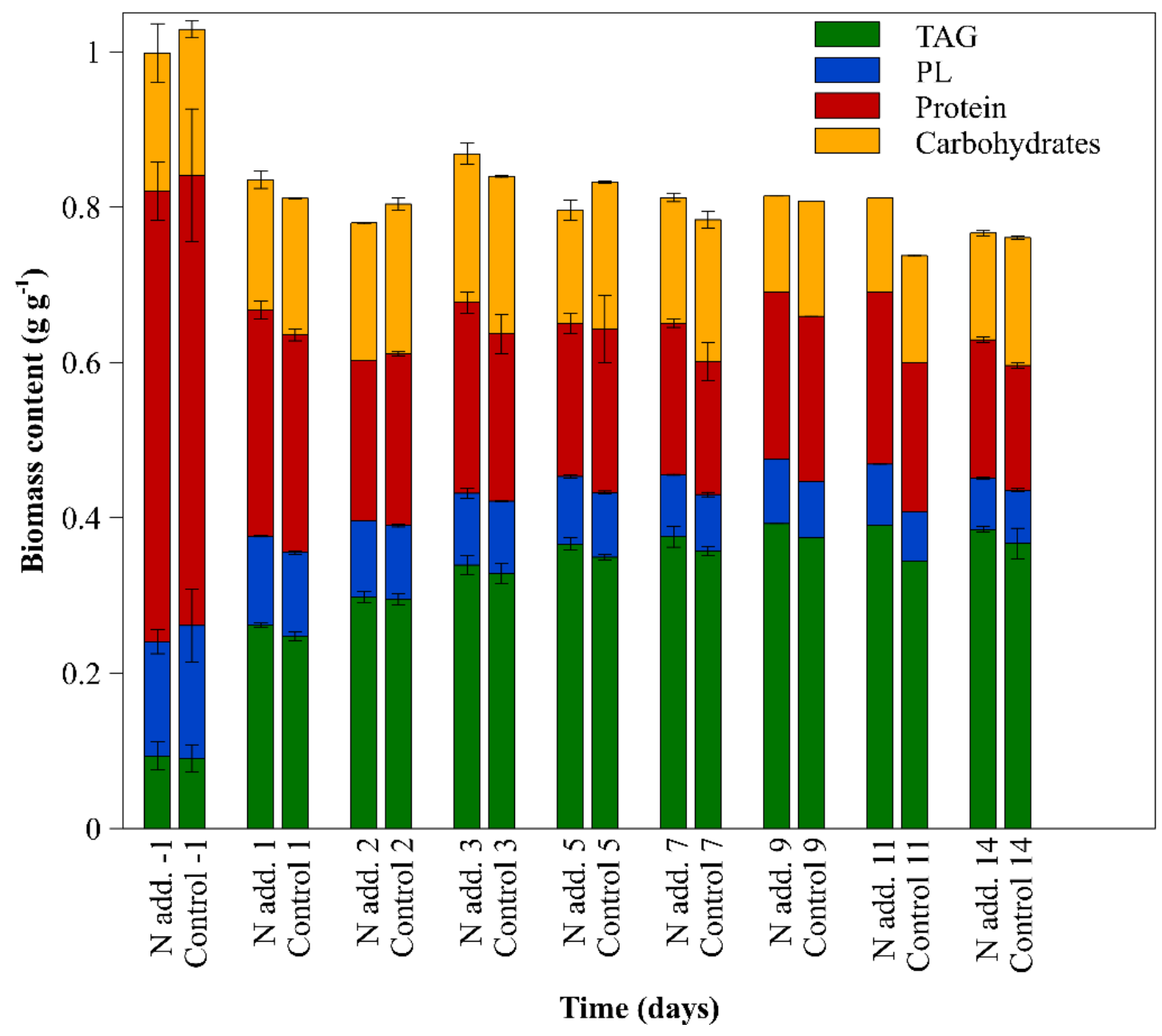

Figure 4.4. Biomass composition in $\mathrm{g} / \mathrm{g}$ dry weight of the cultures with nitrogen addition ( $\mathrm{N}$ add.) and without nitrogen addition (Control) at different days. Measured components were TAG, polar lipids, proteins and total carbohydrates. Error bars show the absolute deviation between the average of the duplicate reactors, except for day 9 and 11 which show data of one reactor.

The biomass composition changed over time when the nitrogen starvation phase started. When comparing the culture with and without nitrogen addition, however, no clear difference in biomass composition was measured between the cultures (Figure 4.4). In general, about $80 \%$ of the biomass composition can be measured as lipids, carbohydrates and proteins. The remaining (ca. 20\%) biomass will consist of pigments, ashes, DNA and RNA. 


\subsubsection{Lipid accumulation}
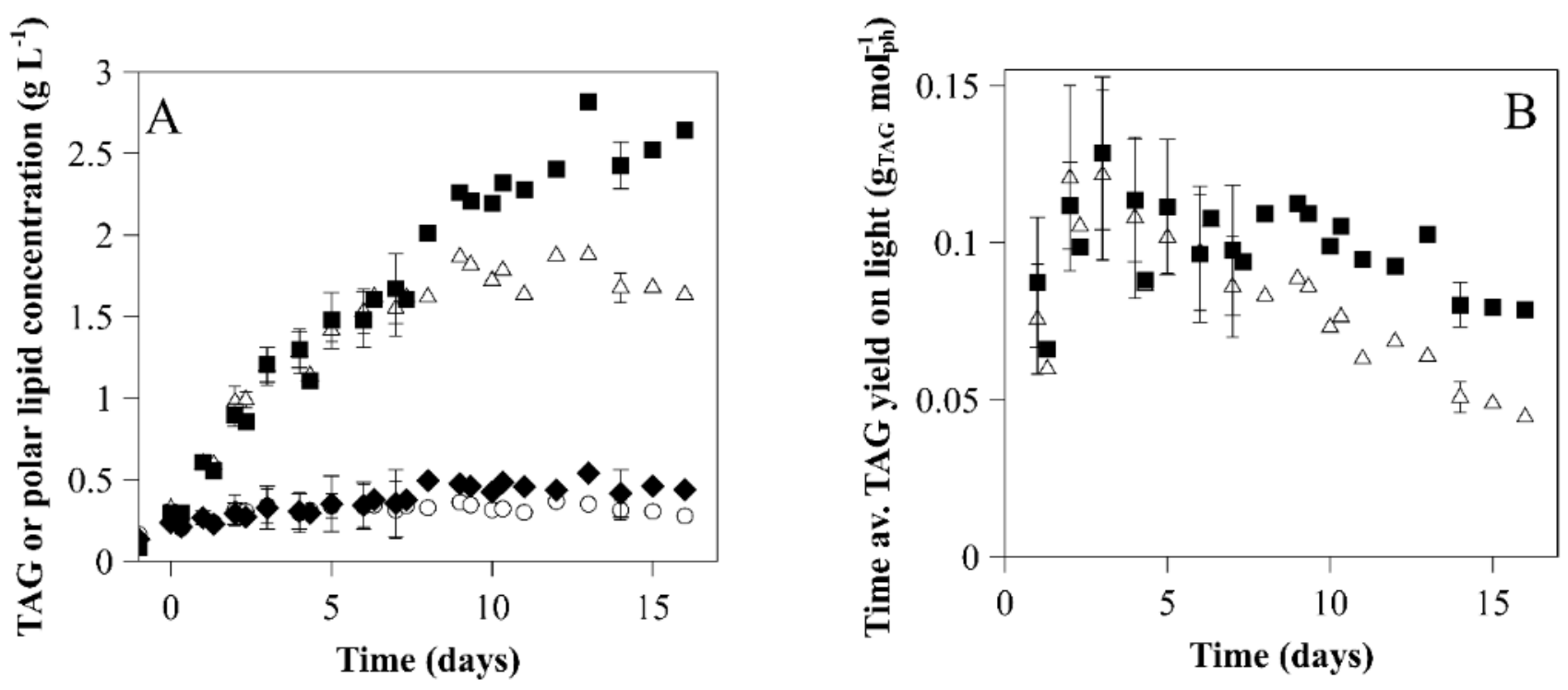

Figure 4.5. Average TAG and polar lipid (PL) concentration $\left(\mathrm{g} \mathrm{L}^{-1}\right)$ over time for the culture with nitrogen addition in the night (TAG: squares and PL: diamonds) and for the control culture without nitrogen addition (TAG: triangles and PL: circles) (A). The time-averaged TAG yield on light $\left(\mathrm{g}_{\mathrm{TAG}} \mathrm{mol}_{\mathrm{ph}}{ }^{-1}\right)$ including a correction for the inoculum production for the culture with nitrogen addition (squares) and without nitrogen addition (triangles) (B). The error bars show the absolute deviation between the averages of the two duplicate experiments. The measurements from day 8 until 13, 16 and 16 show the results of one of the duplicate photobioreactors.

Nitrogen starvation is known to induce TAG accumulation. The TAG content increased rapidly during the first three days of nitrogen starvation up to $33 \%$ of dry weight and kept increasing at a slower rate to a stable value of approximately $40 \%$ of dry weight after 9 days of nitrogen starvation. No difference was found between the culture with and without nitrogen addition (Figure 4.4). However, due to the higher biomass concentration, the TAG concentration in the reactor with nitrogen addition was higher from day 8 onwards (Figure 4.5A). TAG concentration at day 14 was $2.43 \pm 0.14 \mathrm{~g} \mathrm{~L}^{-1}$ and $1.68 \pm 0.09 \mathrm{~g} \mathrm{~L}^{-1}$ for the cultures with and without nitrogen addition, respectively. This shows an increase in TAG accumulation in the culture with nitrogen addition. Since the difference in biomass concentration only starts after the maximal TAG content was reached, the nightly nitrogen addition did not 
improve the maximal TAG content. During the first days of nitrogen starvation the TAG concentration decreased during the night, but no consistent decrease was found. Since no differences in decrease were found between the cultures with and without nitrogen addition, it appears that no extra TAG was used for nitrogen assimilation.

The maximum time-averaged TAG yield on light was similar and reached three days after nitrogen starvation, $0.13 \pm 0.02 \mathrm{~g} \mathrm{~mol} \mathrm{ph}^{-1}$ and $0.12 \pm 0.02 \mathrm{~g} \mathrm{~mol} \mathrm{ph}^{-1}$ for the culture with and without nitrogen addition (Figure 4.5B) (without correction for inoculum production the maximum is $0.18 \pm 0.04 \mathrm{~g} \mathrm{~mol}^{-1}$ after 1 day and $0.19 \pm 0.04$ $\mathrm{g} \mathrm{mol}_{\mathrm{ph}}^{-1}$ after two days for the culture with and without nitrogen addition). This is similar to $0.10 \mathrm{~g} \mathrm{molph}^{-1}$ found for Nannochloropsis sp. with a sinusoidal light dark cycle with same average daily light flux as used in this study (Benvenuti et al., 2016a). From day 8 the time-averaged TAG yield on light was higher for the culture with addition of nitrogen. At day 14 the time-averaged TAG yield on light was $0.08 \pm 0.007$ $\mathrm{g} \mathrm{molph}^{-1}$ and $0.05 \pm 0.005 \mathrm{~g} \mathrm{~mol}^{-1}$ for the culture with and without nitrogen addition, respectively. However, since this only takes place many days after the maximal TAG yield on light was reached, the maximal time-averaged TAG yield was not improved substantially.

The polar lipid content was $12 \%$ at the beginning of the starvation phase and decreased to $6 \%$ of dry weight during nitrogen starvation. The polar lipid concentration was similar for both cultures with and without nitrogen addition (Figure 5A). 


\subsubsection{Protein and total carbohydrate concentration}
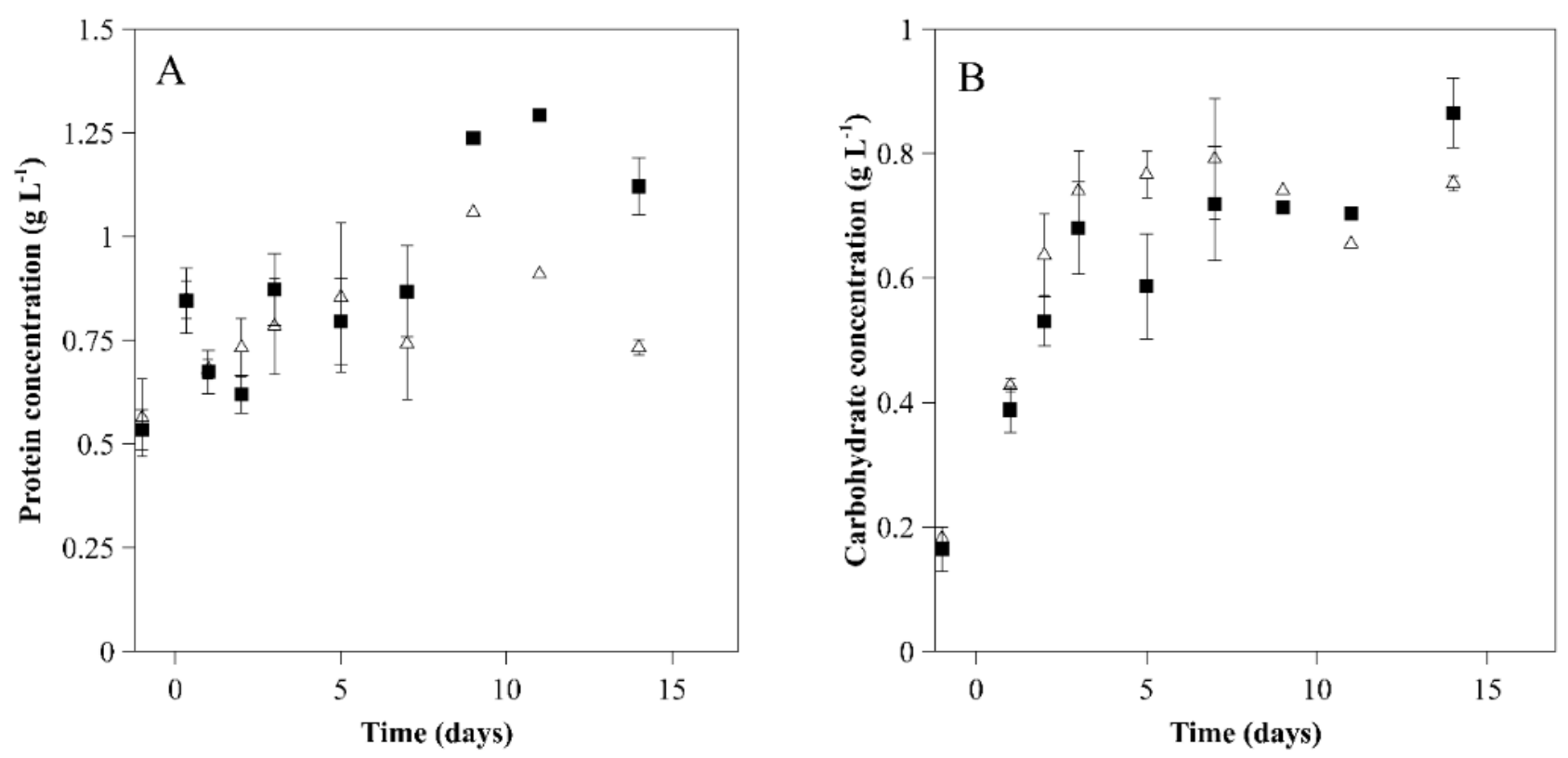

Figure 4.6. Average protein concentration $\left(\mathrm{g} \mathrm{L}^{-1}\right)(\mathrm{A})$ and total carbohydrate concentration ( $\mathrm{g} \mathrm{L}^{-1}$ ) (B) for culture with nitrogen addition (squares) and without (triangles) (averaged from duplicate experiments). Error bars show absolute deviation between the average of the duplicate experiments except for day 9 and 11 which were measurements from one reactor.

The protein content of the biomass one night after the start of nitrogen starvation was $0.48 \pm 0.03$ and $0.49 \pm 0.04 \mathrm{mg} \mathrm{mg}^{-1}$ and it decreased to $0.18 \pm 0.005$ and $0.17 \pm$ $0.004 \mathrm{mg} \mathrm{mg}^{-1}$ at day 14 , for the culture with and without nitrogen addition, respectively (Figure 4.5). The protein concentration in the reactor increased to 1.12 $\pm 0.07 \mathrm{~g} \mathrm{~L}^{-1}$ compared to $0.73 \pm 0.02 \mathrm{~g} \mathrm{~L}^{-1}$ for the culture with and without nitrogen addition, respectively, at day 14 (Figure 4.6A). This suggests that the consumed nitrogen was used for protein production and only a very small fraction was used for chlorophyll $a$ synthesis. Proteins involved in photosynthesis have shown to be made again after nitrogen resupply in Nannochloropsis oceanica (Dong et al., 2013). It was proposed for Dunaliella tertiolecta that the recovery of photosynthesis and chlorophyll content were dependent on protein synthesis (Young and Beardall, 2003). Since, the protein concentration (Figure 6A) was increased and the measured 
PSII quantum yield was higher for the nitrogen added culture it is likely that proteins involved in photosynthesis were made (Meng et al., 2015).

The total carbohydrate concentration in the reactor increased during the first three days from 0.2 to $0.7 \mathrm{~g} \mathrm{~L}^{-1}$ (Figure 4.6B) and was similar for the culture with and without nitrogen addition. So nightly nitrogen addition did not affect the carbohydrate accumulation during nitrogen starvation. Nannochloropsis does not accumulate starch like Acutodesmus obliquus and Chlorella sp. as storage compounds but is assumed to synthesize laminarin and/ or chrysolaminarin (Jia et al., 2015).

\subsubsection{Lipid accumulation strategy}

The current strategy based on addition of low amounts of nitrogen during the night in nitrogen starved cultures only showed an effect after one week of nitrogen starvation. This late effect could be caused by the low amount of nitrogen added. Adding more nitrogen might improve the photosynthetic efficiency faster, i.e. shortly after the beginning of starvation. But if more nitrogen is added it will probably not be consumed during the dark period and a large part will be available during the light period and light will then be used for growth instead of lipid accumulation. In addition, more TAG degradation will take place in similarity to the nitrogen replenished cultures after nitrogen starvation and repeated batch cultures (where part of the culture is harvested during starvation and replaced by fresh medium with nitrogen) (Benvenuti et al., 2016b; Mulders et al., 2014b). Addition of more nitrogen will therefore likely not improve TAG yield on light. In the current study we cannot discriminate if the effect is due to the nitrogen addition during the night or due to the nitrogen addition in general. To study if the improvement was due to the nitrogen addition during the night, or the nitrogen addition in general, similar experiments could be performed where the same amount of nitrogen is added during the day instead of night. To improve the TAG yield on light it is important to keep a high TAG yield for a longer period of time. The nitrogen addition should improve the TAG yield earlier than after 8 days of nitrogen starvation. 


\subsection{CONCLUSIONS}

Nitrogen addition to a nitrogen starved batch culture during the night showed an improvement on the growth and lipid accumulation of $N$. gaditana after 7 days of nitrogen starvation. Nightly addition of nitrogen improved the photosystem II quantum yield after 6 days of nitrogen starvation. The added nitrogen was most likely used for protein synthesis. The maximal time-averaged TAG yield on light was reached after three days of nitrogen starvation and therefore small nightly nitrogen additions did not lead to an increased maximal TAG yield on light. From day 8 onwards the time-averaged TAG yield was higher in the culture with nitrogen addition. 


\subsection{SUPPLEMENT: NITROGEN ADDITIONS}

Table S4.1. Amount of nitrogen added per dry weight $\left(\mathrm{mg} \mathrm{g}^{-1}\right)$ at the start of each dark period during the nitrogen starvation period. Data shown for one of the duplicate experiments.

\begin{tabular}{|c|c|}
\hline Time nitrogen starvation (days) & $\begin{array}{c}\mathrm{N} \text { added per dry weight }\left(\mathrm{mg} \mathrm{g}^{-1}\right) \\
\text { (Experiment } 1)\end{array}$ \\
\hline 0 & 1.5 \\
\hline 1 & 1.4 \\
\hline 2 & 1.3 \\
\hline 3 & 1.2 \\
\hline 4 & 1.5 \\
\hline 5 & 1.3 \\
\hline 6 & 1.1 \\
\hline 7 & 0.9 \\
\hline 8 & 0.9 \\
\hline 9 & 0.9 \\
\hline 10 & 0.8 \\
\hline 11 & 0.6 \\
\hline 12 & 0.5 \\
\hline 13 & 0.4 \\
\hline 14 & 0.5 \\
\hline 15 & 0.4 \\
\hline
\end{tabular}




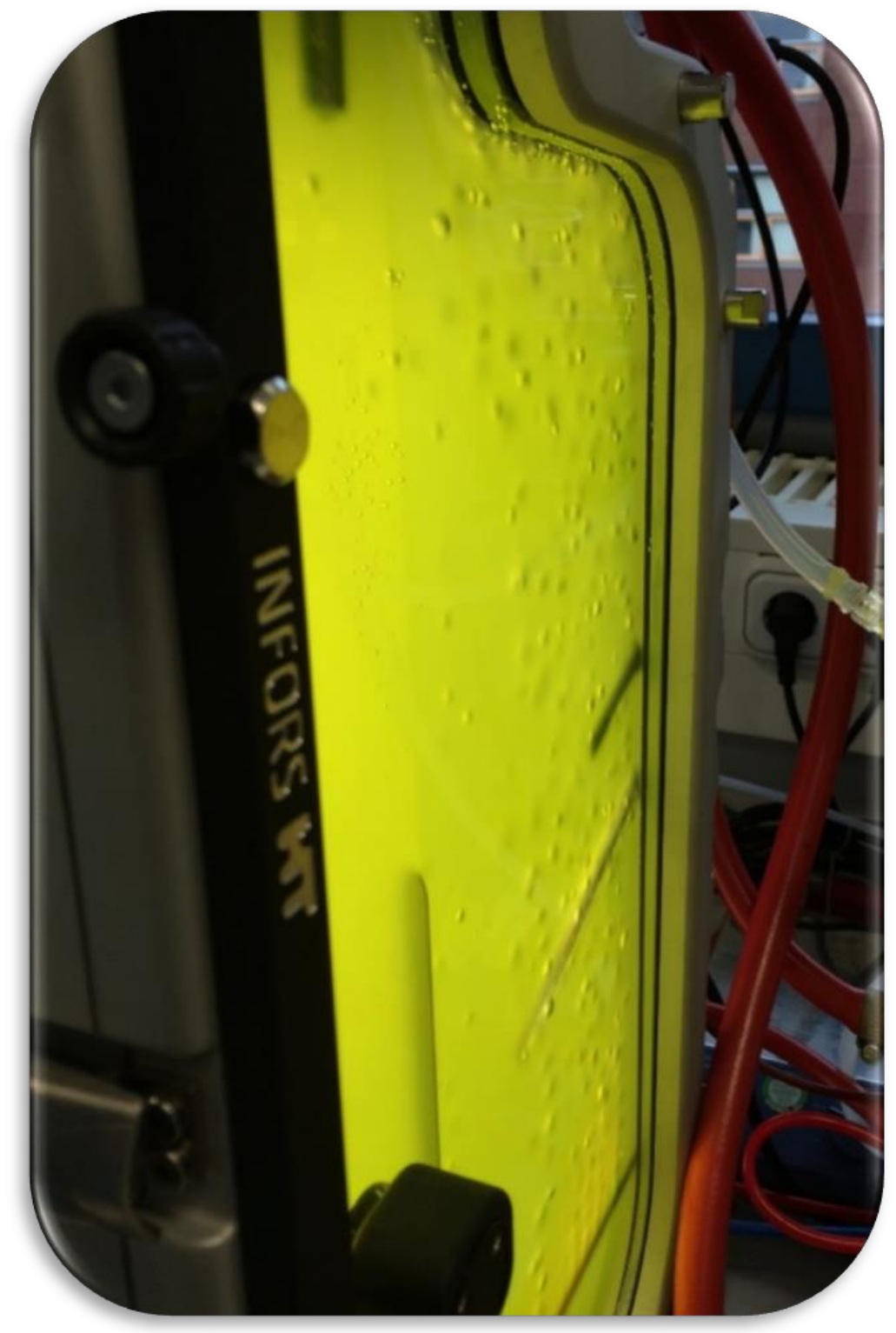

Side view of the photobioreactor 


\section{Chapter 5}

\section{Effect of initial biomass-specific}

photon supply rate on fatty acid accumulation in nitrogen depleted Nannochloropsis gaditana under simulated outdoor light conditions

This chapter is submitted for publication as:

JH Janssen, JLSP Driessen, PP Lamers, RH Wijffels and MJ Barbosa

Effect of initial biomass-specific photon supply rate on fatty acid accumulation in nitrogen depleted Nannochloropsis gaditana under simulated outdoor light conditions 


\section{ABSTRACT}

Triacylglycerol (TAG) accumulation in the microalgae Nannochloropsis gaditana is induced by nitrogen starvation and dependent on the light supplied. We studied under simulated outdoor light conditions the effect of supplied light on the TAG yield by varying the biomass-specific photon supply rate present at the onset of nitrogen starvation. High, intermediate and low average biomass-specific photon supply rates $\left(26,11\right.$ and $\left.6 \mu \mathrm{mol} \mathrm{g}^{-1} \mathrm{~s}^{-1}\right)$ were achieved by applying equal incident light intensity to different biomass concentrations $\left(1.2,2.9\right.$ and $\left.5.4 \mathrm{~g} \mathrm{~L}^{-1}\right)$. The intermediate biomassspecific photon supply rate resulted in the highest time-averaged TAG yield on light; $0.09 \mathrm{~g}_{\mathrm{TAG}} \mathrm{mol}_{\mathrm{ph}}{ }^{-1}$. Sub-optimal yields were attributed to photosaturation, photoinhibition, light falling through reactor without being absorbed and high maintenance requirements. The biomass-specific photon supply rate is important to optimize TAG production by microalgae. 


\subsection{INTRODUCTION}

Due to the increasing world population, the demands for food and energy are rising the need for alternative, sustainable sources for food-commodities and fuels increases (Draaisma et al., 2013). Triacylglycerol (TAG) has a wide range of applications and is used in the food- and petrochemical industry. Microalgae can be used as sustainable alternative for TAG production. For this, the production process should be optimized to be economically feasible and compete with plant-based and fossil oils (Wijffels and Barbosa, 2010). Nitrogen starvation is the most applied technique for TAG accumulation in microalgae (Hu et al., 2008). Nannochloropsis is a marine microalgae species which accumulates large amounts of TAG (up to 54\% per dry weight) upon nitrogen starvation and produces the omega-3 fatty acid eicosapentaenoic acid (EPA) (Benvenuti et al., 2016b; Simionato et al., 2013). Nitrogen starvation is often applied in a two phase batch strategy in which the microalgae are first grown under non-limiting conditions followed by nitrogen starvation to induce TAG accumulation.

Under identical light intensities, low biomass concentration results in a high biomassspecific photon supply rate and a high biomass concentration results in a low biomass-specific photon supply rate. Light supply rate is important as it affects the photosynthetic rate and thereby the biomass and TAG yields. In outdoor cultivation, for a given cultivation system and light intensity, the biomass-specific photon supply rate can be changed by changing the biomass concentration inside the reactor. Biomass concentration dictates the light gradient inside the photobioreactor and, at a fixed light intensity, also the biomass-specific photon supply rate. There are different processes which influence the photosystems and photosynthetic rates: photosaturation, photoinhibition and photoacclimation. When suboptimal biomass-specific photon supply rates are used, these processes can lead to suboptimal TAG yields (Figure 5.1). 


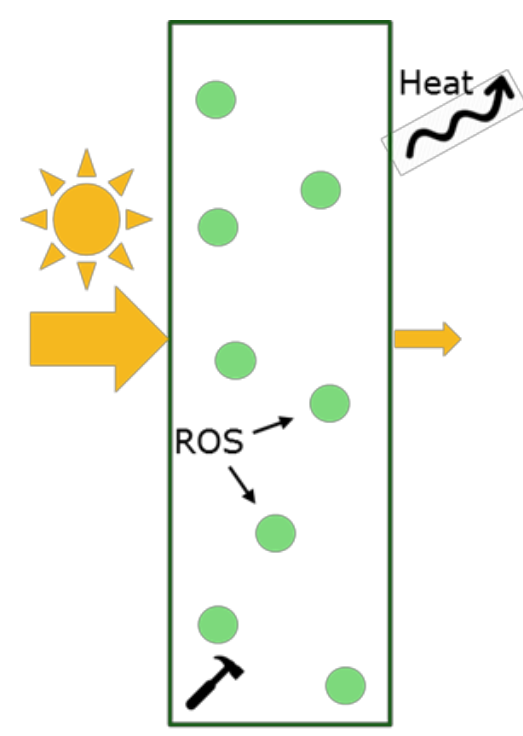

High biomass-specific photon supply $26 \mu \mathrm{mol} \mathrm{g}-1 \mathrm{~s}^{-1}$ (low Cx)

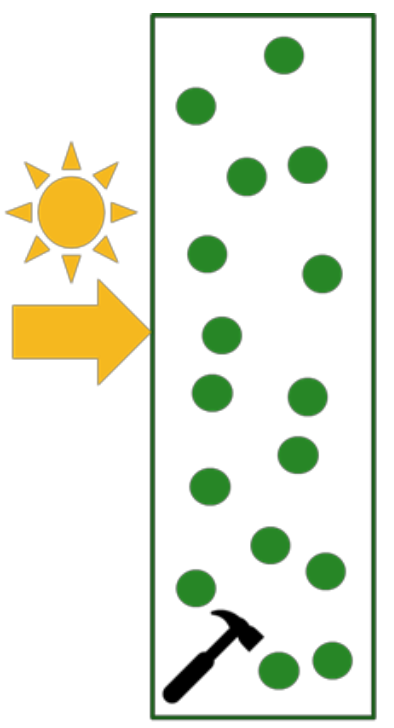

Intermediate biomass-specific photon supply $11 \mu \mathrm{mol} \mathrm{g} \mathrm{g}^{-1} \mathrm{~s}^{-1}$ (intermediate $\mathrm{Cx}$ )

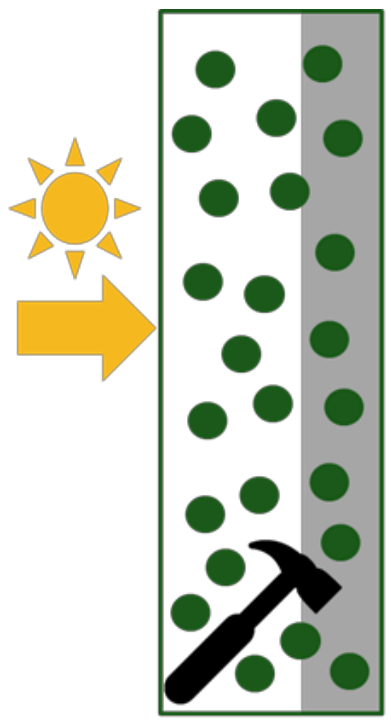

Low biomass-specific photon supply $6 \mu \mathrm{mol} \mathrm{g} \mathrm{m}^{-1} \mathrm{~s}^{-1}$ (high Cx)

Figure 5.1. Schematic overview of different biomass-specific photon supply rates and the processes expected to influence the TAG yield. The size of the hammer represents the volumetric maintenance energy requirement.

One of these process is photosaturation. At light intensities higher than the photosynthetic processing capacity, photosynthesis is saturated and the excess energy is dissipated as heat, thereby decreasing photosynthetic efficiency (Gordon and Polle, 2007). Another process is photoinhibition, which is the process where excess of photons elicit formation of oxygen radicals (ROS) which damage key proteins in the photosynthetic machinery and thereby decrease photosynthetic efficiency and thus increase photosaturation (Nikolaou et al., 2016). Microalgae can also adapt their pigmentation dependent on the light intensity received through photoacclimation and thereby adapt their photosynthetic efficiency (Nikolaou et al., 2016).

In addition to the processes affecting the photosystems and photosynthetic efficiency, TAG yield on light will also be affected by the metabolic processes downstream of light absorption; TAG yield decreases when absorbed light energy is used for other processes than TAG production. For instance, light energy is necessary for cell maintenance, which are non-growth related processes. The volumetric energy requirement for cell maintenance is dependent on the biomass concentration 
(Kliphuis et al., 2010). When there is not enough light energy supplied for maintenance, cells need to degrade storage products such as TAG to generate energy to maintain themselves, leading to a lower TAG yield. Another factor contributing to a low TAG yield on light, but now under high biomass-specific photon supply rates, is the loss of light that goes through the reactor without being absorbed.

Previous outdoor studies showed that the amount of biomass at the start of nitrogen starvation affected TAG yield on light for Chlorella zofingiensis (Feng et al., 2011; Zemke et al., 2013). Increasing initial biomass concentration showed increased lipid productivity for the biomass concentrations tested $\left(0.02,0.15,0.35\right.$ and $\left.0.5 \mathrm{~g} \mathrm{~L}^{-1}\right)$ with a reactor depth of $17 \mathrm{~cm}$ at varying outdoor light conditions (Feng et al., 2011). In lab-scale experiments, no significant effect of biomass-specific photon supply rate at the start of nitrogen starvation on the overall TAG production for Chlorella zofingiensis was found between the tested biomass-specific photon absorption rates 4.7, 3.5 and $2.9 \mu \mathrm{mol} \mathrm{g}^{-1} \mathrm{~s}^{-1}$ using continuous light conditions (Mulders et al., 2014a). For Nannochloropsis oculata a maximum TAG productivity under continuous light conditions was found at $13 \mu \mathrm{mol} \mathrm{g}^{-1} \mathrm{~s}^{-1}$ (Kandilian et al., 2014).

In this research, the effect of the biomass-specific photon supply rate on TAG yield in Nannochloropsis gaditana during nitrogen starvation was studied at lab-scale under simulated outdoor light conditions. Simulated outdoor light conditions were used at lab-scale to be more representative of outdoors conditions. The different biomass-specific photon supply rates were set by applying equal light intensities to different biomass concentrations present at the moment of nitrogen starvation. The outdoor light intensity was simulated using a half-sinus incident light intensity curve with a peak at noon of $1500 \mu \mathrm{mol} \mathrm{m} \mathrm{m}^{-2} \mathrm{~s}^{-1}$ and a day : night cycle of $16: 8$ hours. The average initial biomass-specific photon supply rates were; 26,11 and $6 \mu \mathrm{mol} \mathrm{gdw}^{-1} \mathrm{~s}^{-1}$ at the start of nitrogen starvation. This required initial biomass concentrations of 1.2, 3.0 and $5.4 \mathrm{~g} \mathrm{~L}^{-1}$. It was hypothesized that there is an optimal biomass concentration and thus biomass-specific photon supply rate at the start of nitrogen starvation where the TAG yield on light is maximal. Besides TAG, the omega- 3 fatty acid eicosapentaenoic acid (EPA) was studied in more depth. EPA is a fatty acid present in the photosynthetic membranes and TAG, and therefore differences are expected at different biomass-specific photon supply rates. 


\subsection{MATERIALS AND METHODS}

\subsubsection{Strains, cultivation medium and pre-culture conditions}

The microalgae Nannochloropsis gaditana CCFM-01 was obtained from the Microalgae Collection of Fitoplancton Marino S.L.. Pre-cultures of $N$. gaditana were kept in $250 \mathrm{~mL}$ Erlenmeyer flasks with $100 \mathrm{~mL}$ culture, incubated at $25^{\circ} \mathrm{C}$ in an orbital shaker incubator (125 rpm). The cultures were maintained at low light conditions (30-40 $\left.\mu \mathrm{mol} \mathrm{m} \mathrm{m}^{-2} \mathrm{~s}^{-1}\right)$, in a $16: 8$ hours day : night cycle. A week before inoculation, the microalgae were transferred to continuous high light conditions (118 $\mu \mathrm{mol}$ $\mathrm{m}^{-2} \mathrm{~s}^{-1}$ ) with air enriched with $2.5 \% \mathrm{CO}_{2}$ for inoculum production. The growth medium was based on (Breuer et al., 2012) and contained: $\mathrm{NaCl} 445 \mathrm{mM}$; $\mathrm{KNO}_{3} 33.6 \mathrm{mM}$; $\mathrm{Na}_{2} \mathrm{SO}_{4} 3.5 \mathrm{mM} ; \mathrm{MgSO}_{4} \cdot 7 \mathrm{H}_{2} \mathrm{O} 3 \mathrm{mM}$; $\mathrm{CaCl}_{2} \cdot 2 \mathrm{H}_{2} \mathrm{O} 2.5 \mathrm{mM} ; \mathrm{K}_{2} \mathrm{HPO}_{4} 2.5 \mathrm{mM}$; NaFeEDTA $28 \mu \mathrm{M}$; $\mathrm{Na}_{2} \mathrm{EDTA} \cdot 2 \mathrm{H}_{2} \mathrm{O} 80 \mu \mathrm{M} ; \mathrm{MnCl}_{2} \cdot 4 \mathrm{H}_{2} \mathrm{O} 19 \mu \mathrm{M} ; \mathrm{ZnSO}_{4} \cdot 7 \mathrm{H}_{2} \mathrm{O} 4 \mu \mathrm{M}$; $\mathrm{CoCl}_{2} \cdot 6 \mathrm{H}_{2} \mathrm{O} 1.2$ $\mu \mathrm{M}$; $\mathrm{CuSO}_{4} \cdot 5 \mathrm{H}_{2} \mathrm{O} 1.3 \mu \mathrm{M}$; $\mathrm{Na}_{2} \mathrm{MoO}_{4} \cdot 2 \mathrm{H}_{2} \mathrm{O} 0.1 \mu \mathrm{M}$; Biotin $0.1 \mu \mathrm{M}$; vitamin $\mathrm{B} 13.3 \mu \mathrm{M}$; vitamin $\mathrm{B} 120.1 \mu \mathrm{M}$; and $10 \mathrm{mM} \mathrm{NaHCO}$. For nitrogen depleted growth medium $\mathrm{KNO}_{3}$ was replaced with $33.6 \mathrm{mM} \mathrm{KCl}$ to keep equal osmolarity. Since the nitrate and phosphate concentration in the medium was high enough for a biomass concentration of approximately $5 \mathrm{~g} \mathrm{~L}^{-1}$ extra nitrate and phosphate were added before it became limited during the growth phase. For the intermediate concentration $\left(2.9 \mathrm{~g} \mathrm{~L}^{-1}\right) 2.8 \mathrm{~g} \mathrm{KNO}_{3}$ was added at day 7 of the growth phase. For the high biomass concentration $\left(5.4 \mathrm{~g} \mathrm{~L}^{-1}\right) 2.6 \mathrm{~g} \mathrm{KNO}_{3}$ was added at day 7 and $4.8 \mathrm{~g} \mathrm{KNO}_{3}$ and $0.6 \mathrm{~g} \mathrm{~K}_{2} \mathrm{HPO}_{4}$ were added at day 13 of the growth phase. During pre-cultivation in Erlenmeyer flasks $100 \mathrm{mM}$ 4-(2-hydroxyethyl)piperazine-1-ethanesulfonic acid (HEPES) was added as $\mathrm{pH}$ buffer. The $\mathrm{pH}$ of the growth media was adjusted to $\mathrm{pH} 7.5$ and filter sterilized prior to use (pore size, $0.2 \mu \mathrm{m}$ ).

\subsubsection{Photobioreactor and experimental setup}

Experiments were performed in an aseptic, heat-sterilized, flat-panel, airlift-loop photobioreactor (Labfors 5 Lux, Infors HT, Switzerland, 2010) with a working volume of $1.8 \mathrm{~L}$ and a reactor depth of $20.7 \mathrm{~mm}$. Mixing was provided by aeration of the culture with $1 \mathrm{~L} \mathrm{~min}^{-1}$ filtered air mixed with $2 \% \mathrm{CO}_{2}$. The $\mathrm{pH}$ was maintained at 7.5 by on-demand addition of $2.5 \%(\mathrm{v} / \mathrm{v})$ sulphuric acid. Temperature was controlled at $26{ }^{\circ} \mathrm{C}$ by recirculation of water through a water jacket in direct contact with the cultivation chamber at the back of the reactor and connected to a cryostat. The incident light intensity was controlled and provided by 260 high power LED lights 
$(28 \mathrm{~V}, 600 \mathrm{Watt})$ at the culture side of the reactor. The reactor chamber was isolated to prevent interference of ambient light. Nannochloropsis gaditana was grown in a two-step batch process using growth phase followed by nitrogen starvation to induce TAG accumulation. In the growth phase, microalgae were inoculated at biomass concentration $0.09-0.12 \mathrm{~g} \mathrm{~L}^{-1}$ in nitrogen replete medium. After growth the cells were harvested by centrifugation ( $800 \mathrm{~g}, 25$ minutes) and washed with nitrogen deplete medium to remove the residual nitrogen and re-inoculated in nitrogen depleted medium, starting the nitrogen starvation phase. The microalgae were grown up 2.6, 5.0 and $7.4 \mathrm{~g} \mathrm{~L}^{-1}$ and diluted back to $1.2,2.9$ and $5.4 \mathrm{~g} \mathrm{~L}^{-1}$, respectively, at the beginning of the nitrogen starvation phase. The dilutions were kept as small as possible to prevent a large change in biomass-specific photon supply rate after washing and starting the nitrogen starvation of the culture.

During the growth phase the microalgae were subjected to a $16: 8 \mathrm{~h}$ day:night cycle with constant light intensity during the day (block light cycle). The light intensity was increased stepwise keeping the outgoing light around $30-40 \mu \mathrm{mol} \mathrm{m}^{-2} \mathrm{~s}^{-1}$. Once a light intensity of $636 \mu \mathrm{mol} \mathrm{m} \mathrm{m}^{-2} \mathrm{~s}^{-1}$ was reached, the block light cycle was changed into a sinusoidal light cycle (Equation 1) during the day as used by (Benvenuti et al., 2016a) simulating a summer day in the Netherlands. During the night, 16 to 24 hours after sunrise, no light was supplied. The light was maintained equal throughout the rest of the experiment. This light regime was maintained for at least 1 day during the growth phase before cells were centrifuged to start the nitrogen starvation phase.

$$
I(t)=I_{\max } * \sin \left(\frac{t}{P * \pi}\right)
$$

Equation 5.1. shows the sinusoidal light cycle applied during the day with I (t)

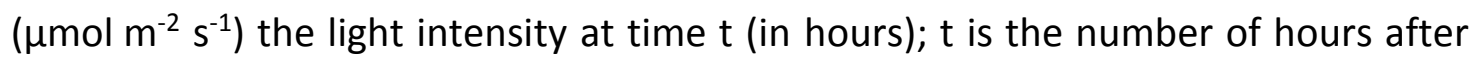
sunrise; Imax is the light intensity at solar noon $\left(1500 \mu \mathrm{mol} \mathrm{m} \mathrm{m}^{-2} \mathrm{~s}^{-1}\right) ; \mathrm{P}$ is the duration of the light period (16 hours). By setting different biomass concentrations at the start of nitrogen starvation the biomass-specific photon supply rate was set. The lowest biomass concentration has the highest biomass-specific photon supply rate. The biomass-specific photon supply rate was calculated by dividing the average light intensity supplied over the light period by the biomass concentration at the start of the nitrogen starvation phase. The initial biomass concentrations and corresponding average biomass-specific photon supply rates at the moment of nitrogen starvation are shown in Table 5.1. 
Table 5.1. Biomass concentration at start of nitrogen starvation and the corresponding average biomass-specific photon supply rate.

\begin{tabular}{|c|c|c|}
\hline $\begin{array}{l}\text { Biomass-specific } \\
\text { photon supply } \\
\text { rate }\end{array}$ & $\begin{array}{c}\text { Biomass concentration } \\
\text { at start of nitrogen } \\
\text { starvation } \\
\left(\mathrm{g} \mathrm{L}^{-1}\right)\end{array}$ & $\begin{array}{c}\text { Average biomass- } \\
\text { specific photon supply } \\
\text { rate } \\
\left(\mu \mathrm{mol}_{\mathrm{ph}} \mathrm{gdw}^{-1} \mathrm{~s}^{-1}\right)\end{array}$ \\
\hline High (a) & 1.2 & 26 \\
\hline Intermediate $(\triangle)$ & 2.9 & 11 \\
\hline Low $(1)$ & 5.4 & 6 \\
\hline
\end{tabular}

\subsubsection{Offline measurements}

During the experiment, daily samples were taken from the photobioreactor to monitor the growth and photosynthetic activity of the microalgal biomass. The optical density (OD) was measured at $750 \mathrm{~nm}$ using a UV-VIS spectrophotometer (Hach Lange DR-6000, light path $1 \mathrm{~cm}$ ). The dry weight was measured in triplicate by filtering (Whatman, $55 \mathrm{~mm}$ ) and drying of biomass samples overnight at $100{ }^{\circ} \mathrm{C}$ as described by (Kliphuis et al., 2012) with the exception that ammonium formate $(0.5 \mathrm{M})$ was used for dilution and washing. Since the supernatant was turbid reddish after centrifugation the dry weight of the supernatant was subtracted from the dry weight and also used for the other analyses. The cell concentration was measured in duplicate with the Multisizer II (Beckman Coulter) using a $50 \mu \mathrm{m}$ aperture tube. Isotone II diluent was used to dilute the samples before measuring.

The photosystem II (PSII) maximal quantum yield $\left(\mathrm{F}_{\mathrm{v}} / \mathrm{F}_{\mathrm{m}}\right)$ was measured by the chlorophyll $a$ fluorescence at $455 \mathrm{~nm}$ using a fluorometer (AquaPen-C AP-C100, Photon Systems Instruments, Czech Republic) and calculated according to (Benvenuti et al., 2015). Samples were diluted to OD750 0.3 and dark-adapted for 15 minutes at room temperature before measurement.

The dry weight-specific optical cross section was measured using a spectrophotometer (Shimadzu UV-2600, Japan), equipped with an integrating sphere, to measure light absorption between $400-750 \mathrm{~nm}$. The dry weight-specific optical cross section $\left(\mathrm{m}^{2} \mathrm{~kg}^{-1}\right.$ ) was calculated according to (de Mooij et al., 2015). Samples were diluted to approximately OD 750 of 0.3 prior to measurement and 
transferred in cuvettes (100.099-OS, Hellma Germany; light path: $2 \mathrm{~mm}$ ). To calculate the volumetric optical cross section, the dry weight-specific optical cross section was multiplied with the dry weight concentration in the photobioreactor.

\subsubsection{Fatty acid analysis}

Biomass samples were centrifuged, washed twice with ammonium formate (0.5 M), stored at $-20{ }^{\circ} \mathrm{C}$ and lyophilized. Lipids were extracted, separated and quantified according to (Breuer et al., 2012; León-Saiki et al., 2017). In short, cells were disrupted using a beat beater and lipids were extracted using a mixture of chloroform:methanol (1:1.25, v:v) with tripentadecanoin (T4257, Sigma Aldrich) and 1,2-Dipentadecanoyl-sn-Glycero-3-[Phospho-rac-(1-glycerol)] (sodium salt) (840446P, Avanti Polar Lipids Inc.) as internal standard for the TAG and polar membrane lipid fraction, respectively. The TAGs were separated from the polar membrane lipids by solid phase extraction (Sep-Pak Vac 6cc, Waters) using different eluents. The fatty acids were methylated by incubation with methanol with $5 \% \mathrm{H}_{2} \mathrm{SO}_{4}$ for 3 hours at $70{ }^{\circ} \mathrm{C}$ and extracted with hexane. The fatty acid methyl esters were identified and quantified using gas chromatography (GC-FID) (Breuer et al., 2012). The total TAG and polar membrane lipid content was calculated as a sum of the individual fatty acids of these fractions.

\subsubsection{Calculation time-averaged TAG yield on light}

The time-averaged TAG yield on light was calculated according to Remmers et al., 2017a. The TAG produced over a certain time period was divided by the light supplied during that period. This light included the light necessary for inoculum production, using a theoretical biomass yield on light of $1 \mathrm{~g} \mathrm{molph}^{-1}$. It is important to consider the energy necessary for inoculum production especially when using high starting biomass concentrations.

\subsection{RESULTS AND DISCUSSION}

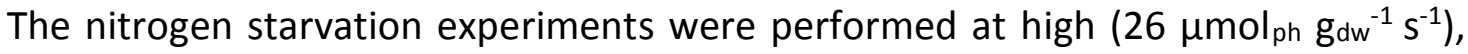
intermediate $\left(11 \mu \mathrm{mol}_{\mathrm{ph}} \mathrm{gdw}^{-1} \mathrm{~s}^{-1}\right)$ and low $\left(6 \mu \mathrm{mol}_{\mathrm{ph}} \mathrm{gdw}^{-1} \mathrm{~s}^{-1}\right)$ biomass-specific photon supply rates by using a low $\left(1.2 \mathrm{~g} \mathrm{~L}^{-1}\right)$, intermediate $\left(2.9 \mathrm{~g} \mathrm{~L}^{-1}\right)$ and high $\left(5.4 \mathrm{~g} \mathrm{~L}^{-1}\right)$ 
biomass concentrations at the start of nitrogen starvation, respectively. All results are shown from the start of nitrogen starvation.

\subsubsection{Biomass concentration}

From the onset of nitrogen starvation, biomass concentration was measured by dry weight ( $\mathrm{g} \mathrm{L}^{-1}$ ) and cell concentration (cells $\mathrm{mL}^{-1}$ ) (Figure 5.2).
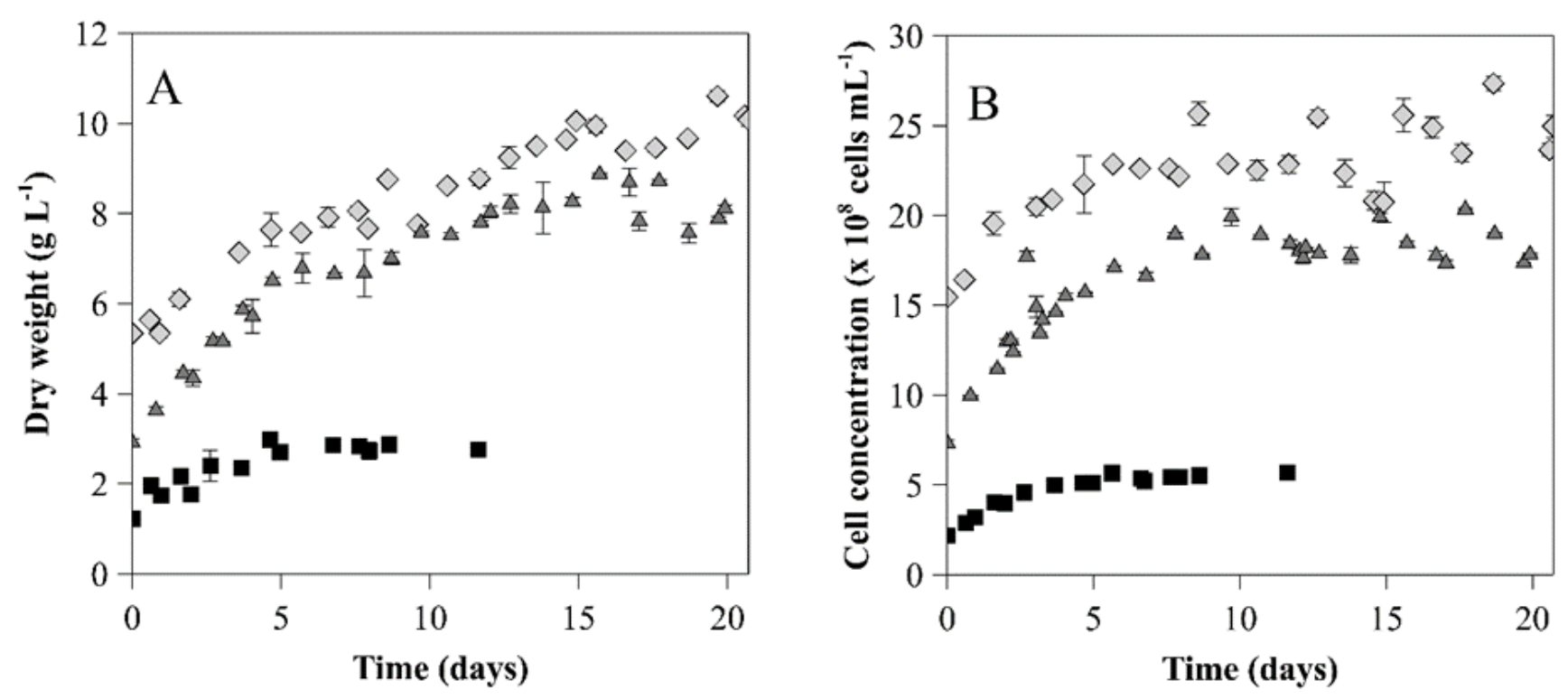

Figure 5.2. Dry weight concentration $\left(\mathrm{g} \mathrm{L}^{-1}\right)(A)$ and cell concentration (cells $\mathrm{mL}^{-1}$ ) (B) for high ( $\left.\square\right)$, intermediate $(\triangle)$ and low ( ) biomass-specific photon supply rate at the start of nitrogen starvation (time $=0$ ). The error bars show the standard deviation of triplicate dry weight measurements and the absolute deviation of duplicate measurements for the cell concentration.

The biomass production rates were on average $0.3,0.8$ and $0.5 \mathrm{gdw}_{\mathrm{L}} \mathrm{L}^{-1} \mathrm{day}^{-1}$ for high, intermediate and low initial biomass-specific photon supply rates, respectively, during the first 5 days of nitrogen starvation (Figure 5.2A). Showing the highest biomass production rate for the intermediate biomass-specific photon supply rate.

Similar to the dry weight results, the intermediate biomass-specific photon supply rate showed the highest increase in cell concentration and thus most cell division during the first 5 days of nitrogen starvation (Figure 5.2B). 


\subsubsection{Lipid accumulation}
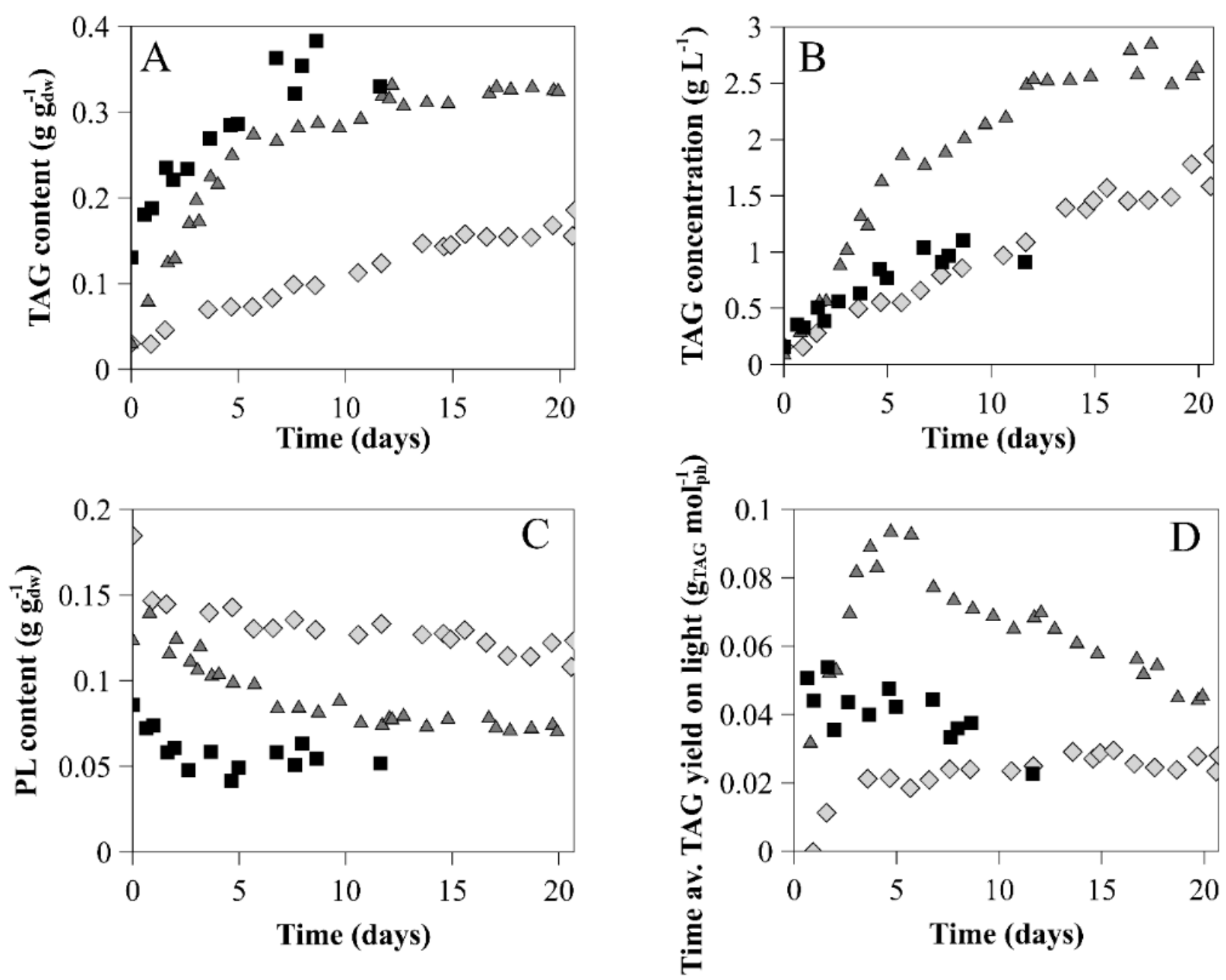

Figure 5.3. TAG content expressed per dry biomass $\left(g_{\mathrm{TAG}} \mathrm{g}_{\mathrm{dw}}{ }^{-1}\right)(\mathrm{A})$, TAG concentration ( $g_{T A G} \mathrm{~L}^{-1}$ ) (B), polar lipid content expressed per dry biomass $\left(g_{P L} g_{d w}{ }^{-1}\right)(C)$ and the time-averaged TAG yield on light ( $g_{T A G}$ molph $^{-1}$ ) (D) for high ( $(\square)$, intermediate $(\Delta)$ and low ( ) biomass-specific photon supply rates at the start of nitrogen starvation (time $=0$ ).

The TAG content at the intermediate biomass-specific photon supply rate increased faster compared to high biomass-specific photon supply rate during the first 5 days of nitrogen starvation (0.05 $\mathrm{g}_{\mathrm{TAG}} \mathrm{g}_{\mathrm{dw}^{-1}}$ day $^{-1}$ compared to $0.03 \mathrm{~g}_{\mathrm{TAG}} \mathrm{g}_{\mathrm{dw}}{ }^{-1} \mathrm{day}^{-1}$ ) (Figure 5.3A). For the low biomass-specific photon supply rate the TAG content increased slower $\left(0.01 \mathrm{~g}_{\mathrm{TAG}} \mathrm{g}_{\mathrm{dw}^{-1}} \mathrm{day}^{-1}\right)$. 
Similar to the increase in TAG content, the TAG concentration in the reactor increased the fastest for the intermediate biomass-specific photon supply rate and reached the highest TAG concentration (Figure 5.3B). The average TAG production rates were $0.12,0.33$ and $0.08 \mathrm{~g} \mathrm{~L}^{-1}$ day $^{-1}$ over the first 5 days of nitrogen starvation for high, intermediate and low biomass-specific photon supply rates.

The polar lipid content was $0.09,0.12$ and $0.18 \mathrm{~g} \mathrm{gdw}^{-1}$ for the high, intermediate and low biomass-specific photon supply rates at the start of nitrogen starvation (Figure 3C). Showing the highest polar lipid content for the lowest biomass-specific photon supply rate.

The maximal time-averaged TAG yield on light was $0.05 ; 0.09$ and $0.02 \mathrm{~g}_{\text {TAG }} \mathrm{mol}_{\mathrm{ph}}{ }^{-1}$ (values not corrected for inoculum production are: $0.07 ; 0.12$ and $0.03 \mathrm{~g}_{\mathrm{TAG}} \mathrm{mol}_{\mathrm{ph}}{ }^{-1}$ ) for high, intermediate and low biomass-specific photon supply rates (Figure 5.3D). These maximal time-averaged TAG yield on light were achieved after 2, 5 and 4 days of nitrogen starvation for the high, intermediate and low biomass-specific photon supply rates, respectively. The highest time-averaged TAG yield on light $\left(0.09\right.$ g $_{\text {TAG }}$ $\mathrm{mol}_{\mathrm{ph}}{ }^{-1}$ ) was achieved at the intermediate initial biomass-specific photon supply rate $\left(11 \mu \mathrm{mol}_{\mathrm{ph}} \mathrm{gdw}_{\mathrm{dw}} \mathrm{s}^{-1}\right)$. This maximal time-averaged TAG yield on light was similar to $0.10 \mathrm{~g}_{\mathrm{TAG}} \mathrm{mol}_{\mathrm{ph}}{ }^{-1}$ achieved for Nannochloropsis sp. cultivated under the same sinusoidal day night light regime using a biomass-specific photon supply rate of 16 $\mu \mathrm{mol}_{\mathrm{ph}} \mathrm{gdw}_{\mathrm{dw}} \mathrm{s}^{-1}$ (Benvenuti et al., 2016a). This is biomass-specific photon supply rate between the high and intermediate biomass-specific photon supply rates used in this

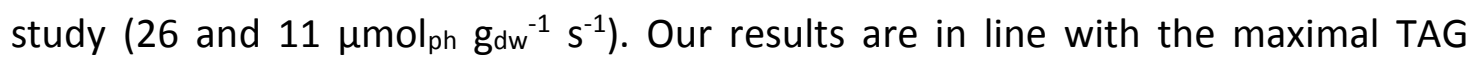
productivity which was achieved for Nannochloropsis oculata at a biomass-specific supply rate of $13 \mu \mathrm{mol} \mathrm{g} \mathrm{g}^{-1} \mathrm{~s}^{-1}$ under continuous light conditions (Kandilian et al., 2014). On the contrary, Mulders et al. 2014a found no difference in TAG yield on light for Chlorella zofingiensis within the tested range of biomass-specific photon absorption rates $\left(2.9,3.5\right.$ and $\left.4.7 \mu \mathrm{mol} \mathrm{g}^{-1} \mathrm{~s}^{-1}\right)$ under continuous light. In the present study, however, a broader biomass-specific photon supply rate range $(6,11$ and 26 $\mu \mathrm{mol} \mathrm{gdw}^{-1} \mathrm{~s}^{-1}$ ) and simulated outdoor light conditions were used. Therefore, suboptimal yields for both lower and higher biomass-specific photon supply rates could be measured. 


\subsubsection{Photosynthetic capacity}

Nitrogen starved Nannochloropsis gaditana relies on photosynthesis to support de novo TAG synthesis (Simionato et al., 2013). Therefore, the photosystem II (PSII) maximum quantum yield and the dry weight-specific optical cross section were measured (Figure 5.4).
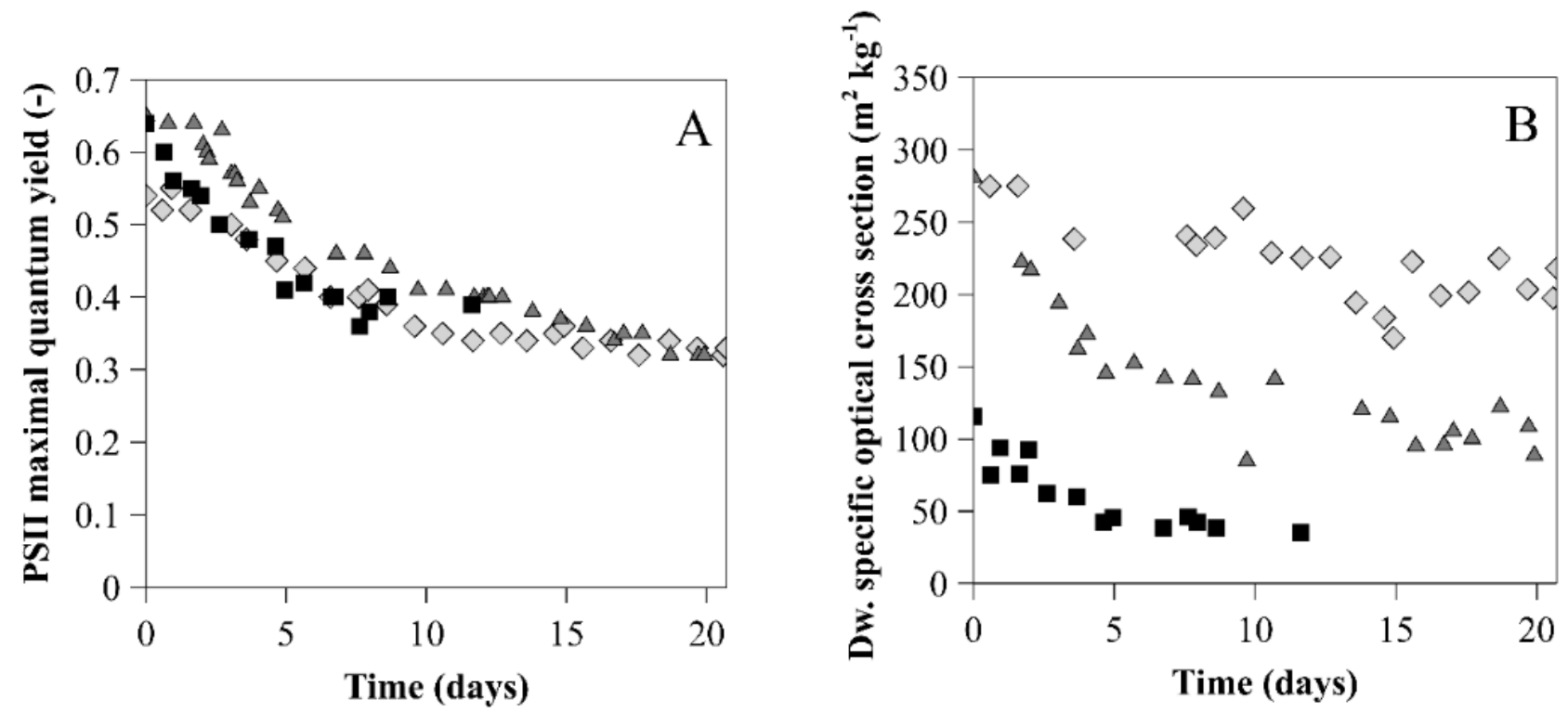

Figure 5.4. Photosystem II maximal quantum yield (A) and dry weight-specific optical cross section $\left(\mathrm{m}^{2} \mathrm{~kg}^{-1}\right)(B)$ for high (E), intermediate $(\triangle)$ and low ( ) biomass-specific photon supply rate at the start of nitrogen starvation (time $=0$ ).

The photosystem II (PSII) maximal quantum yield decreased for all cultures during nitrogen starvation (Figure 5.4A). This was similar to results found for Nannochloropsis oceanica during nitrogen starvation (Dong et al., 2013; Meng et al., 2015) and indicated a reduced photosynthetic efficiency. At the start of nitrogen starvation the PSII maximal quantum yield was similar for the intermediate and high biomass-specific photon supply rates (between 0.64 and 0.67 ) and lower for the low biomass-specific photon supply rate (0.54).

The PSII maximal quantum yield decreased similarly for the low and intermediate biomass-specific photon supply rate. The high biomass-specific supply rate, however, decreased faster indicating more degradation or damage to PSII causing decrease in photosynthetic efficiency (Figure 4A). 
The intermediate biomass-specific photon supply rate resulted in the highest timeaveraged TAG yield on light because the highest photosynthetic efficiency was maintained and all light supplied to the culture was absorbed resulting in a more efficient use of energy for TAG production. Furthermore, the biomass concentration was low enough to keep the energy required for maintenance relatively low compared to the culture with high biomass concentration (low biomass-specific photon supply rate).

The high biomass-specific photon supply rate resulted in lower TAG yield on light compared to the intermediate biomass-specific photon supply rate. This suboptimal yield could be caused by several phenomena. Firstly, the fast decrease in PSII maximal quantum yield can probably be attributed to photoinhibition (Han et al., 2000; Rubio et al., 2003). Photoinhibition is a process in which excess of photons damage key proteins in the photosynthetic machinery resulting in lower photosynthetic efficiency causing suboptimal TAG yield on light. At high biomassspecific photon supply rate can result in the largest excess of photons. Secondly, at the higher biomass-specific photon supply rate photosaturation can occur in a larger part of the reactor compared to the lower biomass-specific photon absorption rates and therefore dissipating more excess energy as heat. Thirdly, part of the supplied incident light fell through the reactor and was not absorbed by the microalgae and could not be used for TAG production. Approximately $8 \%$ of the incident light fell through the reactor at the peak light intensity $\left(1500 \mu \mathrm{mol} \mathrm{m} \mathrm{m}^{-2} \mathrm{~s}^{-1}\right)$ at the first day, without being used by the culture. At low and intermediate biomass-specific photon supply rates all supplied light was absorbed.

The lower TAG yield on light for the low biomass-specific photon supply rate, on the other hand, was probably caused by the relative high volumetric maintenance energy requirement due to high biomass concentration, assuming a constant maintenance energy per biomass for all different biomass concentrations. This energy is not available for TAG production and can therefore lead to a lower TAG yield on light. High biomass concentrations necessary for low biomass-specific supply rate also have a higher energy requirement for inoculum production which has a negative effect on overall TAG yield on light. In addition, the culture with low biomass-specific photon supply rate showed a reduced photosynthetic quantum yield at the start of nitrogen starvation, which can result in lower TAG yields on light. 
The different biomass-specific photon supply rates also influence the dry weightspecific optical cross section (Figure 5.4B). Microalgae can adapt their pigmentation dependent on the light received by photoacclimation. At high biomass-specific photon supply rates (low biomass concentration) the pigmentation decreases and at low biomass-specific photon supply rates (high biomass concentration) the pigmentation increases (Meneghesso et al. 2016). The effect of photoacclimation during the growth phase was shown by the dry weight-specific optical cross section at the start of the nitrogen starvation phase. The biomass-specific optical cross section for the high biomass-specific photon supply rates was lower $\left(116 \mathrm{~m}^{2} \mathrm{~kg}^{-1}\right)$ compared to the low and intermediate biomass-specific photon supply rates ( 275 and $\left.281 \mathrm{~m}^{2} \mathrm{~kg}^{-1}\right)$.

During nitrogen starvation phase, the dry weight-specific optical cross section decreased for all cultures (Figure 5.4B). The volumetric optical cross section $\left(\mathrm{m}^{2} \mathrm{~L}^{-1}\right)$ (Figure S5.6, supplements) was, however, stable for the low and intermediate biomass-specific photon supply rates and increased slightly for at high biomassspecific photon supply rate. The decrease in dry weight-specific optical cross section is therefore caused by the increased of non-absorbing biomass like TAG and thus thereby decreasing optical cross section per biomass. The small increase in volumetric optical cross section for the low biomass-specific photon supply rate was probably due to the increase in biomass concentration and thereby increased pigmentation by photoacclimation.

Photoacclimation most likely results in an increase of photosynthetic membranes. At low biomass-specific photon supply rate the highest dry weight-specific optical cross section results in the highest membranes and thus highest polar lipids at the start of the nitrogen starvation phase (Figure 5.3C). 

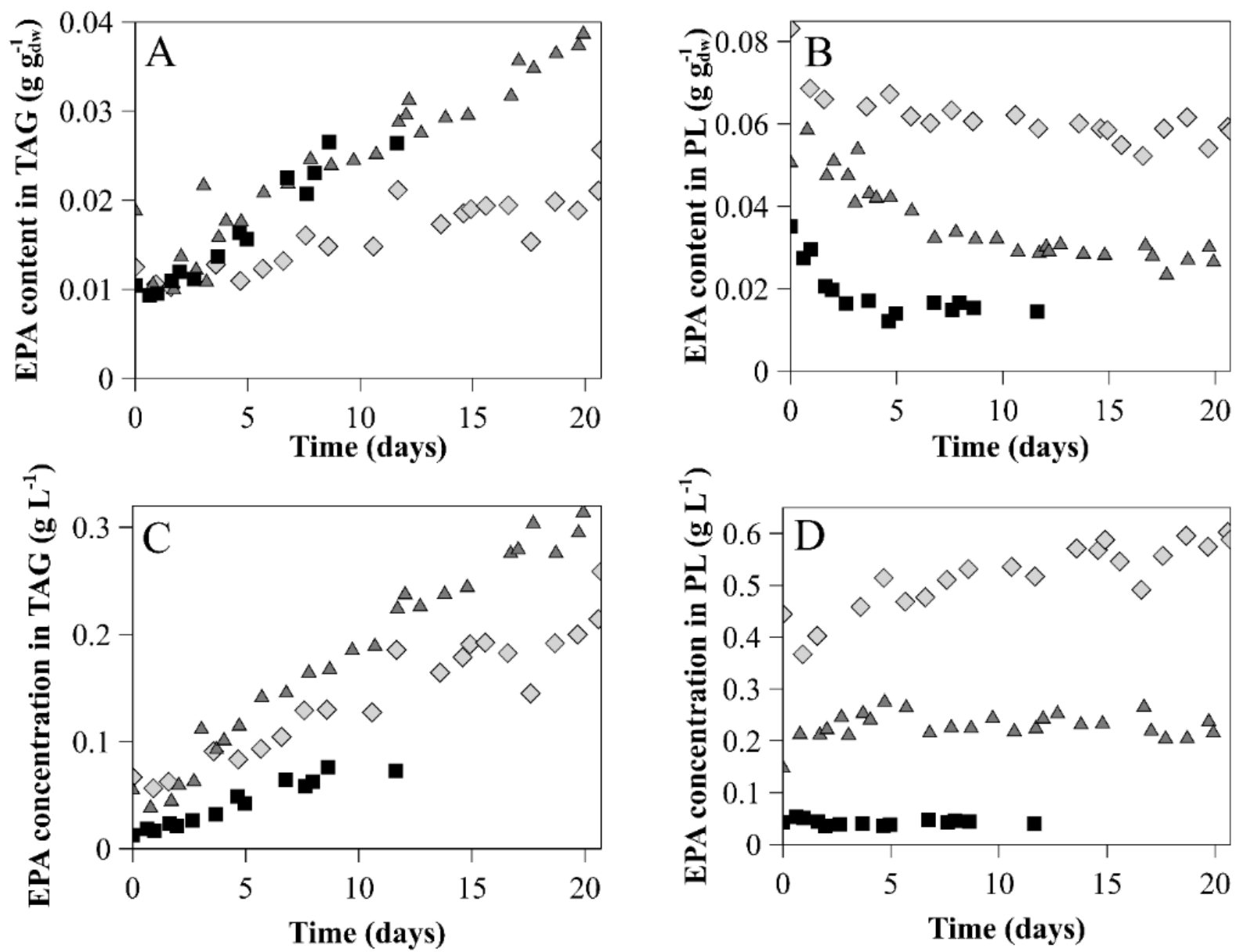

Figure 5.5. EPA content in TAG (A) and polar membrane lipids (PL) (B) expressed per dry biomass ( $\mathrm{g} \mathrm{gdw}^{-1}$ ) and EPA concentration in TAG (C) and $\mathrm{PL}(\mathrm{D})$ fraction $\left(\mathrm{g} \mathrm{L}^{-1}\right)$ from the start of nitrogen starvation for high $(\square)$, intermediate $(\triangle)$ and low ( ) biomass-specific photon supply rate at the start of nitrogen starvation (time $=0$ ).

EPA is one of the main fatty acids present in the polar lipid fraction. EPA content expressed per dry weight in the polar lipid fraction shows the same pattern as the total polar lipid content expressed per dry weight for the different biomass-specific photon supply rates used (Figure 5.5). The EPA content in the polar lipid was the highest at low biomass-specific photon supply rate at start of nitrogen starvation probably due to photoacclimation. Interestingly, the EPA content in TAG expressed per dry weight was similar at start of nitrogen starvation for all biomass-specific photon supply rates used and increased faster for the high and intermediate biomass-specific photon supply rate. The average EPA production in both TAG and 
polar lipid fraction over the first 12 days of nitrogen starvation was 4.9, 20.9 and 16.4 $\mathrm{mg} \mathrm{L}^{-1}$ day $^{-1}$ for the high, intermediate and low biomass-specific photon supply rates, respectively. The average EPA production rate was highest at the intermediate biomass-specific due to the increase of EPA in TAG. EPA production during nitrogen starvation is thus dependent on the initial biomass-specific photon supply rate used.

\subsection{CONCLUSIONS}

The biomass-specific photon supply rate is an important parameter to optimize TAG yield on light during nitrogen starvation. Different biomass-specific photon supply rates were obtained by using different biomass concentration at equal simulated outdoor incident light intensities, at the start of nitrogen starvation. The highest TAG yield was obtained at intermediate biomass-specific photon supply rate due to highest photosynthetic capacity and all supplied light was absorbed. Sub-optimal yield at high biomass-specific photon supply rate was attributed to non-absorbed light, photosaturation and photoinhibition. Oppositely, sub-optimal yield at low biomass-specific photon supply rate was attributed to high maintenance requirements. 


\subsection{SUPPLEMENT}

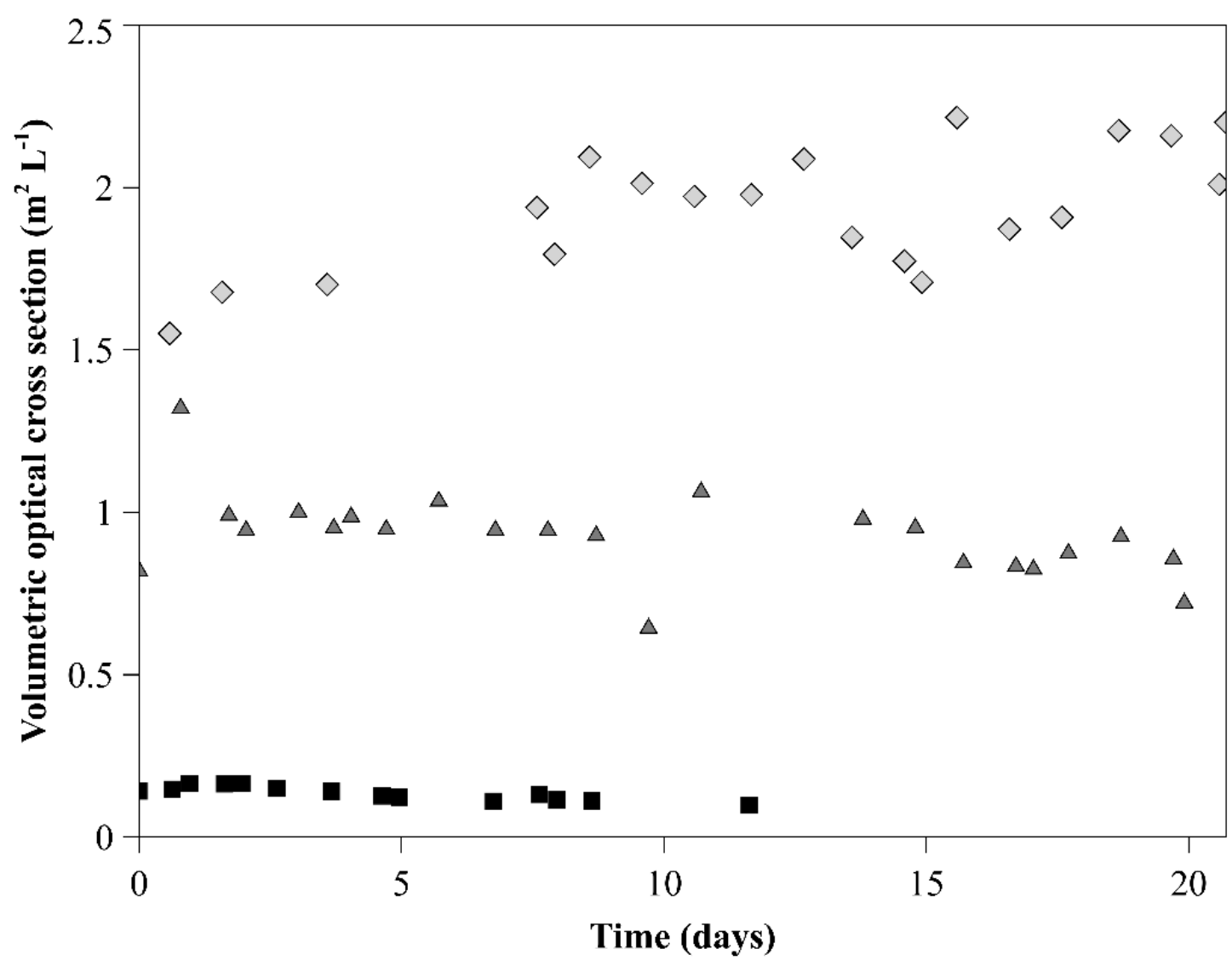

Figure S5.6. Volumetric optical cross section $\left(\mathrm{m}^{2} \mathrm{~L}^{-1}\right)$ from the start of nitrogen starvation for high $(\square)$, intermediate $(\Delta)$ and low ( ) concentration at the start of nitrogen starvation (time $=0$ ). 
Effect of initial biomass-specific photon supply rate during nitrogen starvation 


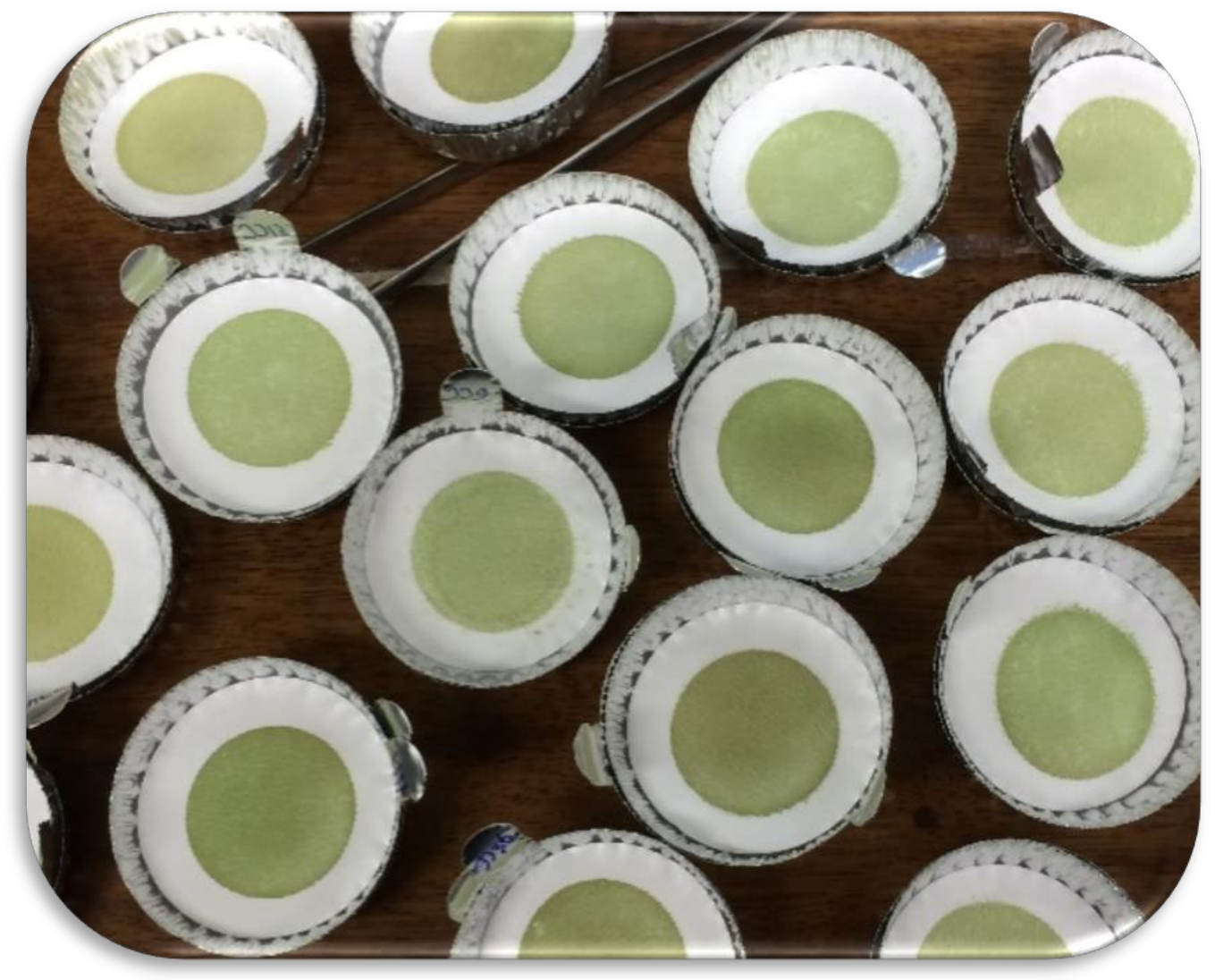

Microalgae dried on filters to measure the dry weight concentration 


\title{
Chapter 6
}

\section{General discussion}

\author{
Lipid production in
}

Nannochloropsis gaditana

This chapter is submitted for publication as:

JH Janssen, RH Wijffels and MJ Barbosa

Lipid production in Nannochloropsis gaditana 


\section{ABSTRACT}

The microalga Nannochloropsis gaditana is a natural producer of triacylglycerol (TAG) and the omega-3 fatty acid eicosapentaenoic acid (EPA). TAG accumulation is induced by nitrogen starvation. The biomass-specific photon supply rate used has an effect on EPA and TAG accumulation during nitrogen starvation as well as, on the localization of EPA accumulation. Clear differences in TAG yield on light are found for different biomass-specific photon supply rates and light regimes. De novo EPA synthesis or translocation of EPA between lipid fractions might be limiting for EPA accumulation in TAG. Further studies are needed to fully understand EPA accumulation in TAG during nitrogen starvation. To elucidate the function of EPA in TAG nitrogen recovery experiments are suggested. Overexpression of genes involved in de novo EPA synthesis and translocation are proposed to elucidate the exact metabolic routes involved in these processes during nitrogen starvation. Showing several future opportunities to increase EPA accumulation. 


\subsection{INTRODUCTION}

Microalgae are known for their ability to produce large amounts of lipids and they are the primary producers of the omega-3 fatty acid eicosapentaenoic acid (EPA), However, this fatty acid is present in small concentration in the cells, up to $4.3 \%$ EPA on dry weight basis in Nannochloropsis gaditana (Ma et al., 2016). Higher values are required to make this process competitive.

Nannochloropsis gaditana is a microalga species known for its capacity to accumulate large amounts of triacylglycerol (TAG) and to produce EPA. One of the most used strategies to induce TAG accumulation is nitrogen starvation. This strategy is often applied as a two-phase batch cultivation, starting with a growth phase followed by nitrogen starvation, in which TAG is produced de novo and by conversion of membrane lipids (Hu et al., 2008). The acyl-CoA dependent pathway is used for de novo synthesis of TAG (Liu and Benning, 2013) and polar membrane lipids can be converted to TAG via the acyl-CoA independent pathway. In general, TAG contains higher amounts of saturated fatty acids and membranes contain higher amounts of polyunsaturated fatty acids (PUFAs), like EPA (Mühlroth et al., 2013). During nitrogen starvation, however, EPA accumulation in TAG increases (Remmers et al., 2017a; Tonon et al., 2002) Chapters 2, 3 and 5. It is hypothesized that PUFAs are stored in TAG during nitrogen starvation to allow for rapid incorporation in plastid membranes upon more favourable growth conditions. In Chapter $\mathbf{2}$ translocation of intact EPA from the polar lipids to TAG was proven using labelled carbon. We have shown that in addition to translocation, EPA is de novo synthesized during nitrogen starvation.

To be able to increase EPA concentration in TAG, a better understanding of the accumulation mechanisms and its regulation are necessary (Schüler et al., 2017) alongside knowledge on the impact of cultivation condition. In research by Mulder et al. 2014a different biomass-specific photon supply rates were tested for Chromochloris zofingiensis showing no large differences in TAG yield on light. Despite light being the most important and often limiting substrate in microalgae photosynthetic processes, the effect of biomass-specific photon supply rates on TAG and EPA accumulation during nitrogen starvation for Nannochloropsis gaditana was unknown.

Different chapters in this thesis describe experiments using various light intensities and initial biomass concentrations, resulting in various initial average biomass- 
specific photon supply rates. In addition, the light regime (day:night cycle and continuous light) was varied. In Chapter 5, it was shown that the biomass-specific photon supply rate influenced TAG and EPA accumulation. The effect of a wide range of conditions on EPA and TAG accumulation during nitrogen starvation can bring more insight on the mechanisms involved and allow identification of limiting steps on TAG and EPA accumulation and ultimately its improvement.

\subsection{Light regime and specific photon supply rate}

In this thesis, Nannochloropsis gaditana was always grown in the same airlift-loop flat-panel photobioreactor with a reactor depth of $20.7 \mathrm{~mm}$ with identical temperature $\left(26^{\circ} \mathrm{C}\right)$ and $\mathrm{pH}(7.5)$. The incident light intensity, light regime and initial biomass concentration were varied resulting in different initial average biomassspecific photon supply rates. Figure 6.1 shows the average biomass-specific photon supply rate at the start of nitrogen starvation for the different experiments. Fatty acid accumulation in TAG and polar lipids was measured in all experiments.

The average incident light intensity used was the same for all chapters except for Chapter 4 (Figure 6.1). The biomass-specific photon supply rate of Chapters 5 and $\mathbf{2}$ were similar; $26 \mu \mathrm{mol} \mathrm{gdw}{ }^{-1} \mathrm{~s}^{-1}$ and $24 \mu \mathrm{mol} \mathrm{gdw}{ }^{-1} \mathrm{~s}^{-1}$, respectively, but the light regime was different. In Chapter 5 light was supplied as a day:night cycle in a half sinus function instead of continuous. In Chapter 4 nitrogen was added during the night. 
Biomass concentration Light intensity
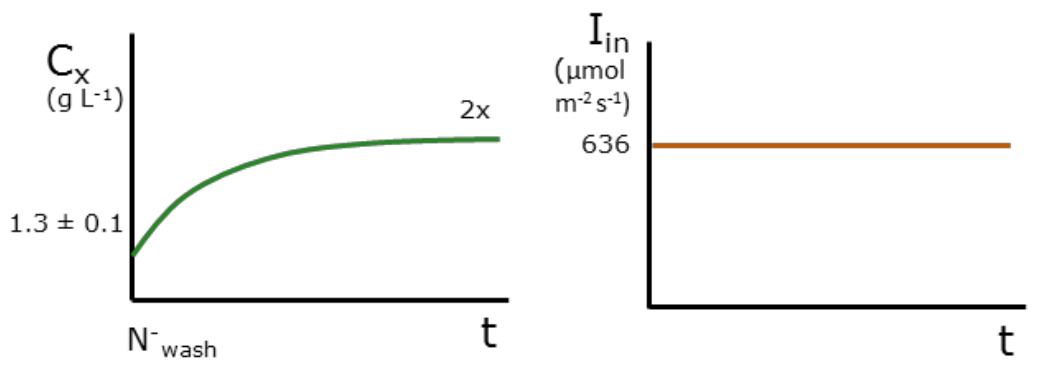

\section{Chapter 2}

${ }^{13} \mathrm{C}$-isotope

$24 \mu \mathrm{mol} \mathrm{gdw}^{-1} \mathrm{~s}^{-1}$
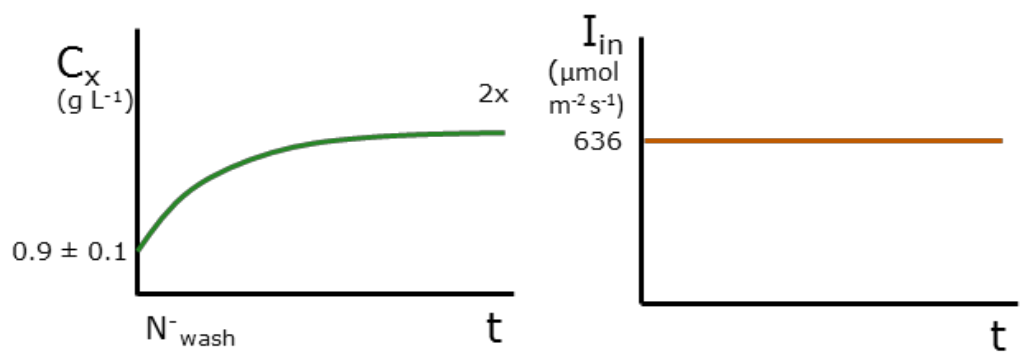

\section{Chapter 3}

Transcriptome

$34 \mu \mathrm{mol} \mathrm{g} \mathrm{dw}^{-1} \mathrm{~s}^{-1}$
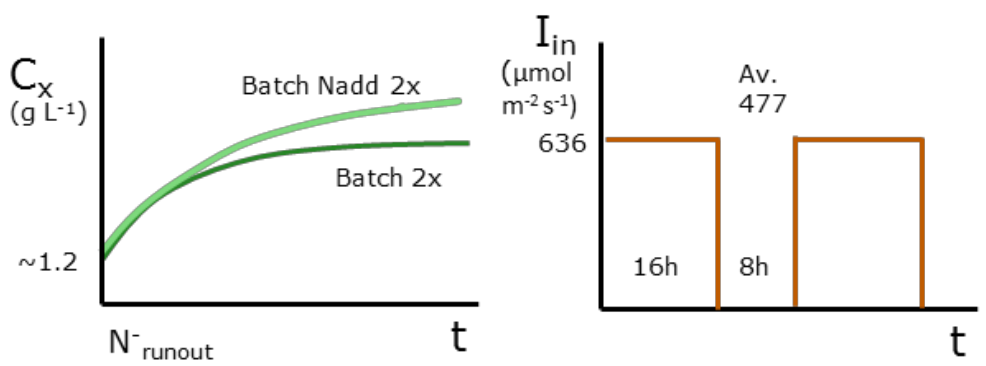

\section{Chapter 4}

Nightly nitrogen

addition

$19 \mu \mathrm{mol} \mathrm{g} \mathrm{dw}^{-1} \mathrm{~s}^{-1}$
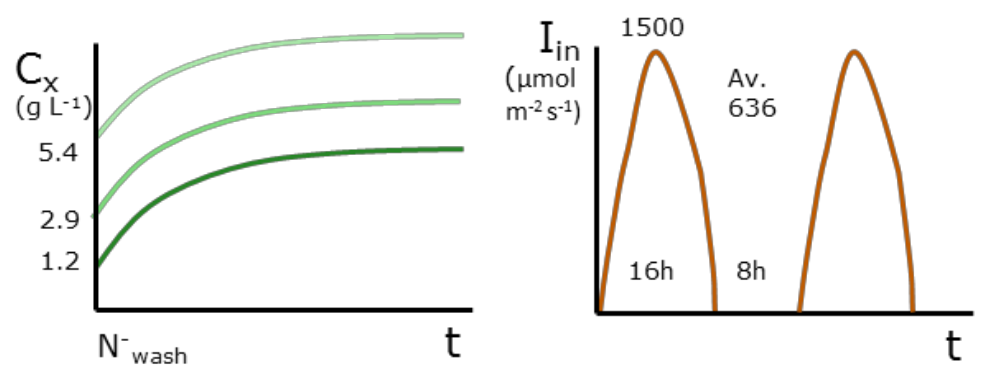

\section{Chapter 5}

Biomass-specific photon supply rate

26, 11 and 6 $\mu \mathrm{mol} \mathrm{g} \mathrm{dw}^{-1} \mathrm{~s}^{-1}$

Figure 6.1. Overview experimental conditions used in the different experiments of this thesis; light regime, average light intensity per day $\left(\mu \mathrm{mol} \mathrm{m} \mathrm{m}^{-2} \mathrm{~s}^{-1}\right)$ and biomass concentration $\left(\mathrm{g} \mathrm{L}^{-1}\right)$ at the onset of nitrogen starvation with their respective average initial biomassspecific photon supply rates $\left(\mu \mathrm{mol} \mathrm{gdw}^{-1} \mathrm{~s}^{-1}\right)$ at the start of nitrogen starvation. 


\subsection{TRIACYLGLYCEROL (TAG)}

\subsubsection{Time-averaged TAG yield on light}

The maximal time-averaged TAG yield on light reached for the different biomassspecific photon supply rates during nitrogen starvation is shown in Figure 6.2. The maximal time-averaged TAG yields on light changes over time are shown in Figure S6.6 for the different experiments.

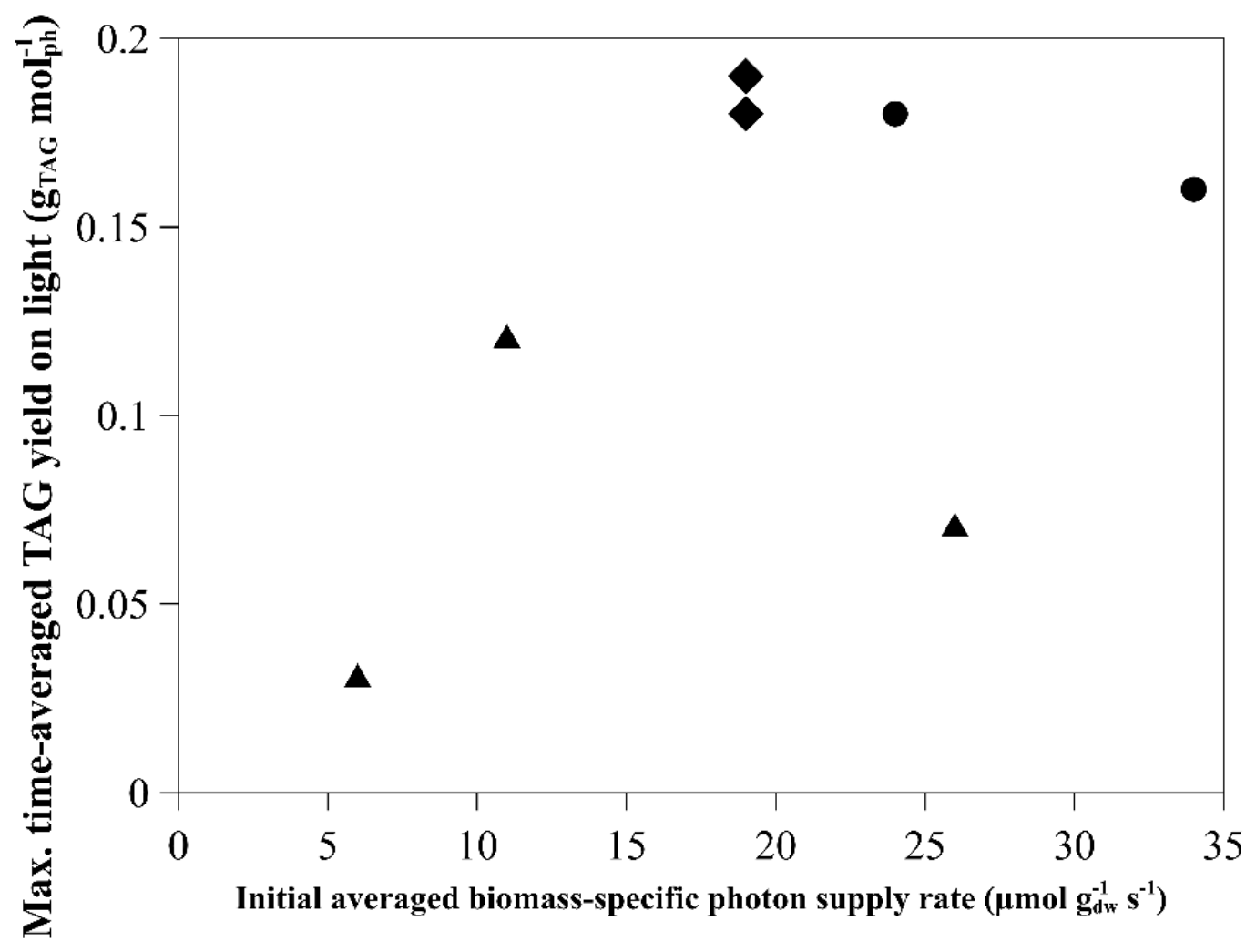

Figure 6.2. Maximal time-averaged TAG yield on light ( TAG $_{\text {Tolph }}{ }^{-1}$ ) reached in the experiments at the initial averaged biomass-specific photon supply rates as shown in Figure 6.1. The light regime used: continuous (circles), sinus (triangles) and block (diamonds).

The highest maximal time-averaged TAG yield on light was reached at a biomass specific photon supply rate between 19 to $24 \mu \mathrm{mol} \mathrm{gdw}^{-1} \mathrm{~s}^{-1}$, in which light intensity was constant and provided continuously or as day:night cycle (Figure 6.2, circles and diamonds). The TAG yields on light achieved under sinus light (Figure 6.2, triangles) were lower compared to the other experiments. Even though similar biomass- 
specific photon supply rates were used ( 24 and $\left.26 \mu \mathrm{mol} \mathrm{gdw}^{-1} \mathrm{~s}^{-1}\right)$ the maximal timeaveraged TAG yield on light achieved was different, 0.18 compared to $0.07 \mathrm{~g}_{\mathrm{TAG}} \mathrm{mol}_{\mathrm{ph}}^{-1}$ for continuous and sinus light conditions, respectively. Besides the biomass-specific photon supply rate, the light regime also influences TAG yield. The lower yield for the sinus light conditions might be the result of a high peak in light intensity $\left(1500 \mu \mathrm{mol} \mathrm{m} \mathrm{m}^{-2} \mathrm{~s}^{-1}\right)$ which can cause photosaturation and photoinhibition, resulting in energy waste as heat and damage of the photosystem, and loss of light going through the reactor without being absorbed (Chapter 5). It should be noted that the TAG content for the sinus light conditions was higher at the start of nitrogen starvation compared to continuous light ( 0.13 compared to $\left.0.08 \mathrm{~g}_{\mathrm{TAG}} \mathrm{g}_{\mathrm{dw}}{ }^{-1}\right)$. This indicates that stress was experienced before nitrogen starvation in this culture, probably as result of the high peak in light intensities.

Since in the experiment with constant light intensity applied as day:night cycle a lower average light intensity was used, no conclusive statement can be made on the effect of constant light intensity applies as day:night cycle compared to continuous light or sinus light. To distinguish the effect of a lower biomass-specific photon supply rate from the effect of the day:night light regime an experiment with the same biomass-specific photon supply rate as provided with continuous light ( $24 \mu \mathrm{mol} \mathrm{gdw}^{-1} \mathrm{~s}^{-1}$ ) should be used and the nitrogen starvation should be started by washing the culture. This higher biomass-specific photon supply rate can be achieved by keeping the same incident light intensity and lowering the initial biomass concentration or by keeping the same initial biomass concentration and increase the incident light intensity.

The achieved maximal time-averaged TAG yields on light were compared to other microalgal species using different biomass-specific photon supply rates and light regimes (Figure 6.3). The growth conditions; initial biomass concentration, light intensity, light regime and the resulting biomass-specific photon supply rates for the different species with their references are given in Table S6.1. 


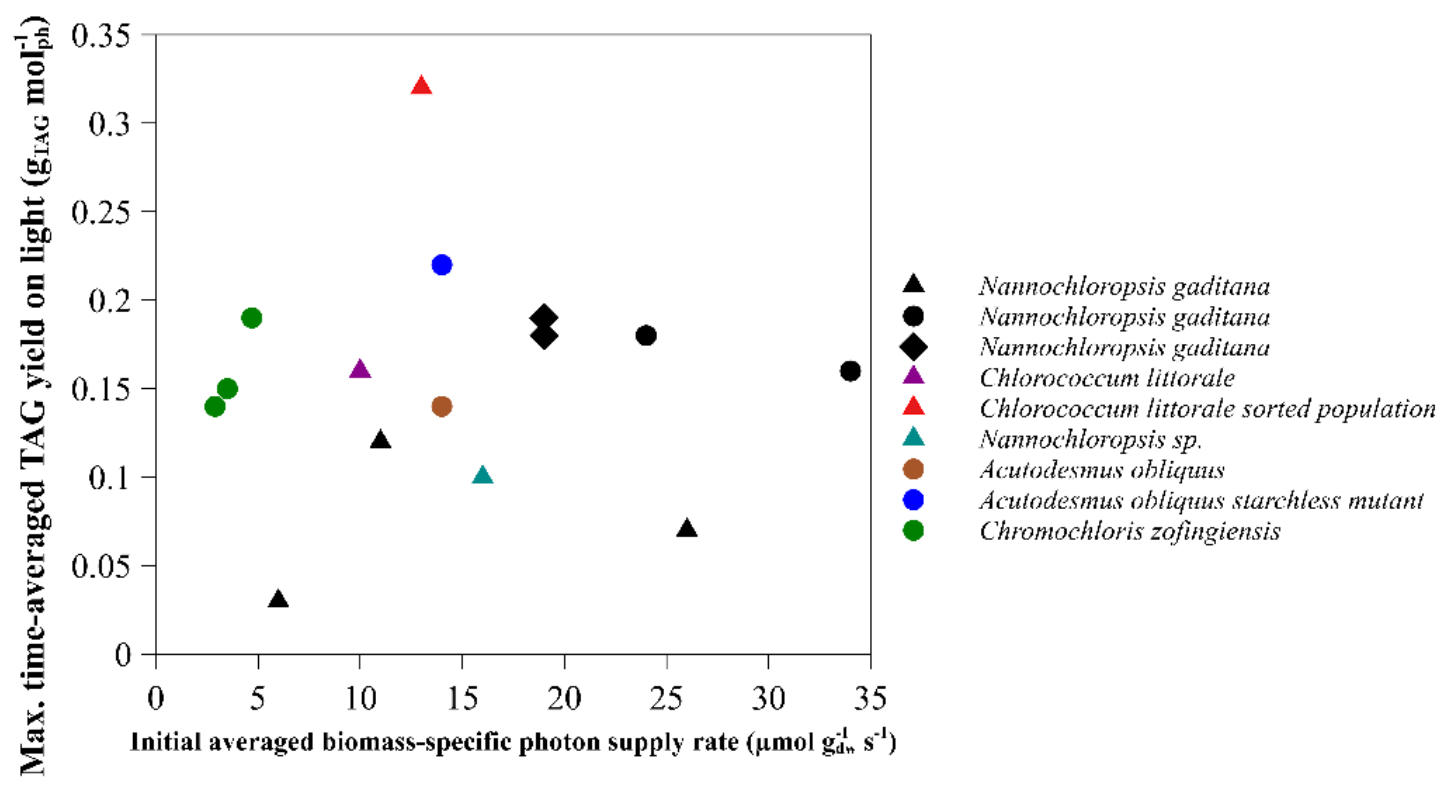

Figure 6.3. Maximal time-average TAG yield on light comparison for various microalgal species under various initial biomass-specific photon supply rates. The light regime used: continuous (circles), sinus (triangles) and block (diamonds). The yield for Chromochloris zofingiensis was calculated based on absorbed light. Details of the calculated biomass-specific photon supply rates from different studies are shown in Table S6.1.

No clear trend was found in the maximal time-averaged TAG yields on light for different microalgal species subjected to different biomass-specific photon supply rates at the onset of nitrogen starvation. The TAG yield on light during nitrogen starvation is not only dependent on the biomass-specific photon supply rate and light regime used but also on the microalgal species used. This should be considered for TAG optimization. The sorted population of Chlorococcum littorale showed the highest maximal time-averaged TAG yield on light indicating that cell sorting can be an interesting approach also for Nannochloropsis gaditana to improve TAG yield (Cabanelas et al. 2016). 


\subsubsection{TAG content}

The TAG content expressed per dry weight was measured at the start of the nitrogen starvation and after 14 days of nitrogen starvation for all different biomass-specific photon supply rates and light regimes used (Figure 6.4A).
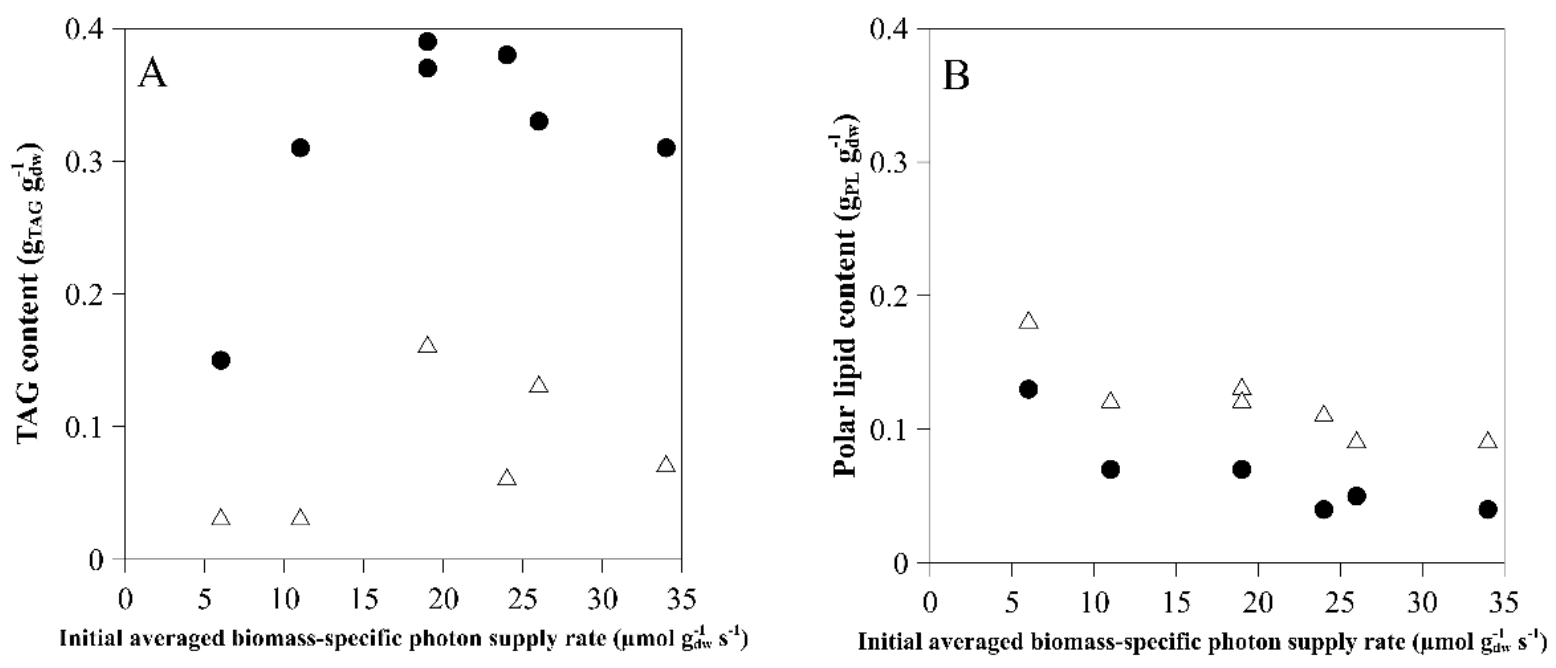

Figure 6.4. TAG (A) and polar lipid (B) content $\left(g_{T A G} g_{d w}{ }^{-1}\right)$ at the start of nitrogen starvation (triangles) and after 14 days (12 days for 26 $\mu \mathrm{mol} \mathrm{gdw}^{-1} \mathrm{~s}^{-1}$ ) of nitrogen starvation (circles) for the different average biomass-specific photon supply rates $\left(\mu \mathrm{mol} \mathrm{gdw}^{-1} \mathrm{~s}^{-1}\right.$ ) at the onset of nitrogen starvation.

The highest TAG contents expressed per dry weight $\left(0.41 \mathrm{~g}_{\mathrm{TAG}} \mathrm{g}_{\mathrm{dw}}{ }^{-1}\right)$ was reached for $19 \mu \mathrm{mol} \mathrm{g} \mathrm{dw}^{-1} \mathrm{~s}^{-1}$ but similar maximal TAG contents were reached for most biomassspecific photon supply rates. This suggests that there might be a limit in the amount of TAG that can be accumulated in the cell. A minimum cell volume might be necessary for basic cell functioning resulting in a maximum volume of TAG which can accumulate. There is no current knowledge on this, but it might be an important factor to consider to increase lipid productivity, alongside with how it is determined which maximum cell diameter that can be obtained. If there is a maximal volume in the cell for TAG accumulation it can be advantageous having cell division in the first days after nitrogen starvation, or having a (mutant) strain which can produce TAG without impairing growth (Ajjawi et al., 2017). Other possibilities are selecting for larger cells with a relative lower minimum required cell volume for basic cell functioning or selecting cells with higher TAG productivity (Cabanelas et al. 2016). 
Nannochloropsis gaditana still divided on average 1.5 times in the first days after nitrogen starvation.

A much lower TAG content $\left(0.19 \mathrm{~g}_{\mathrm{TAG}} \mathrm{g}_{\mathrm{dw}}{ }^{-1}\right)$ and consequently a lower TAG yield on light was reached with a biomass-specific light supply rate of $6 \mu \mathrm{mol} g_{d w}{ }^{-1} s^{-1}$. Due to a low biomass-specific photon supply rate there might be insufficient energy to induce large TAG accumulation. This can be caused by a low incident light intensity or by high volumetric maintenance energy requirements due to the high biomass concentration. To study whether energy is indeed limiting, extra energy could be added during the nitrogen starvation phase by increasing light intensity. If this results in increased TAG accumulation, light was limiting.

\subsection{POLAR LIPIDS}

TAG can be synthetized de novo and via conversion of membrane lipids, which consist mainly of polar lipids. Therefore, the effect of light conditions on polar lipid content was as well studied (Figure 6.4B). In all cultures the polar lipid content expressed per dry weight decreased from the moment of nitrogen starvation. The polar lipid contents at the start of the nitrogen starvation phase were negatively correlated with the biomass-specific photon supply rate; the lowest biomass-specific photon supply rate resulted in the highest polar lipid content. This is probably the result of photoacclimation, the process in which the photosystem increases and possibly polar membrane lipids, due to the low light conditions (Chapter 5 ). The polar lipids decreased less than the increase in TAG, indicating part of the TAG was made de novo.

\subsection{EICOSAPENTAENOIC ACID (EPA)}

The fatty acid EPA accumulates both in the polar lipids in the membranes and in TAG lipid bodies. Figure 6.5 shows the EPA contents expressed per dry weights in both lipid fractions at the start and after 14 days of nitrogen starvation. The change of the EPA content expressed per dry weight in TAG, polar lipids and in total lipids (TAG + $\mathrm{PL}$ ) for all days during nitrogen starvation is shown in Figure S6.7. 


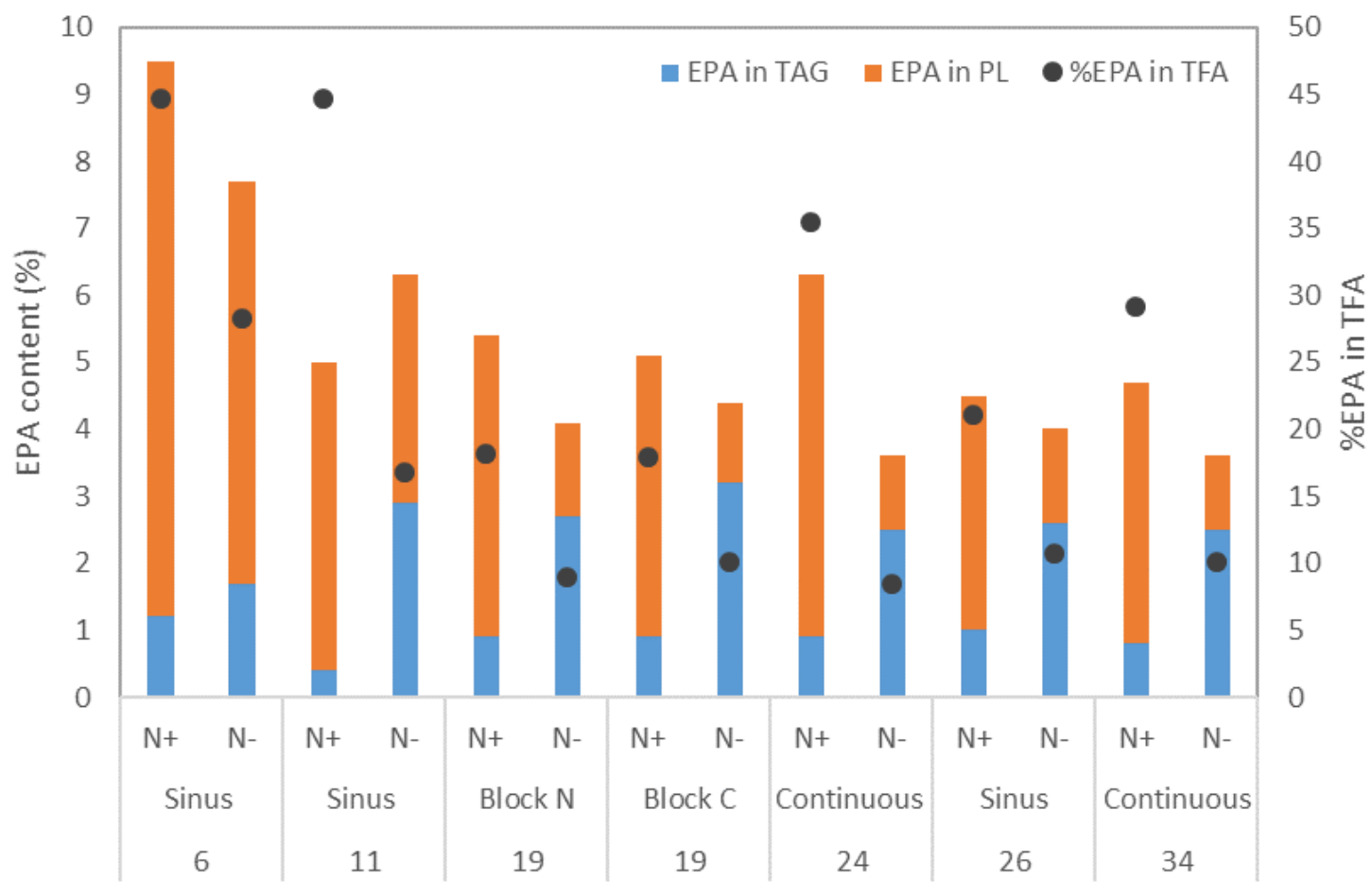

Figure 6.5. EPA content in TAG and polar lipids (PL) expressed as percentage of dry weight and the EPA percentage of the total fatty acids (TFA) for the different initial biomass-specific photon supply rates and light regimes. Results are shown at the start of the nitrogen starvation phase $(\mathrm{N}+)$ and after 14 days of nitrogen starvation (12 days for $\left.26 \mu \mathrm{mol} \mathrm{gdw}^{-1} \mathrm{~s}^{-1}\right)(\mathrm{N}-)$.

The highest EPA content was reached with the lowest biomass-specific photon supply rate at the onset of nitrogen starvation. This high EPA content was mainly due to the EPA present in polar lipids. At low biomass-specific photon supply rates, more membranes are formed as a result of photoacclimation leading to higher polar lipids and consequently EPA contents in the cell. The high EPA content at the start of the nitrogen starvation phase is interesting for EPA production, however, it should be noted that the growth phase took much longer compared to other experiments ( 21 days compared 7 to 11 days), to obtain a high biomass concentration.

The total EPA content expressed per dry weight decreased slightly in most experiments during nitrogen starvation. But as a result of the increased biomass concentration the total EPA concentration in the reactor increased (Figure S6.8). This shows that part of the EPA has to be produced de novo during nitrogen starvation as 
confirmed with labelling study in Chapter 2. In all experiments the EPA percentage of total fatty acids (TFA) decreased during nitrogen starvation due to the faster increase of other fatty acids in TAG, mainly palmitic acid and palmitoleic acid.

The distribution of EPA in the cell showed that EPA content in TAG was similar for all cultures at the start of nitrogen starvation (Figure 6.5, $\mathrm{N}+$ bars). During nitrogen starvation the EPA content in TAG increased and after 14 days of nitrogen starvation a large fraction of all EPA in the cell was present in the TAG lipid fraction. For most conditions the fraction of all EPA in the cell, present in TAG was 60 to $70 \%$, except for the cultures with the lowest biomass-specific photon supply rates (sinus light 11 and $6 \mu \mathrm{mol} \mathrm{gdw}^{-1} \mathrm{~s}^{-1}$ ) which were $50 \%$ and 25\%, respectively (Figure S6.9). One possible strategy to increase the amount of EPA in the cell during nitrogen starvation, is by increasing the amount of EPA accumulated in TAG since it can accumulate up to large contents.

A better understanding of the function of EPA accumulation can help finding targets to increase its accumulation. It is hypothesized that EPA accumulation in TAG acts as storage for membrane components to be rapidly used upon better growth conditions. In Parietochloris incisa, a radiolabelling study showed that arachidonic acid present in TAG was used for chloroplast membrane lipid upon nitrogen recovery (Khozin-Goldberg et al., 2005). To conclude whether EPA is stored in TAG by Nannochloropsis gaditana during nitrogen starvation for membrane synthesis upon nitrogen recovery, labelling experiment similar to Chapter 2 should be done. An initial growth phase should be done followed by nitrogen starvation with labelled carbon $\left({ }^{13} \mathrm{C}\right)$ and concluded by a third phase in which nitrogen is re-supplied and the carbon source is changed to ${ }^{12} \mathrm{C}$ carbon. The newly synthesized fatty acids or translocated fatty acids from TAG to the membranes during recovery phase can in be distinguished by measuring the ${ }^{12} \mathrm{C}$ and ${ }^{13} \mathrm{C}$ fatty acids. This will provide an answer to the hypothesis that TAG acts as a storage for plastid material during nitrogen starvation.

In Chapter 2, it was shown that $23 \%$ of the EPA accumulated in TAG was intact translocated from the polar lipids during nitrogen starvation and $21 \%$ of the EPA accumulated in TAG was completely made de novo. Since de novo synthesis, translocation and carbon cycling cannot be distinguished from only fatty acids data, no conclusions can be drawn on its distribution under different biomass-specific photon supply rates. Both translocation and de novo synthesis are important for EPA 
accumulation in TAG. Possible limiting factors for accumulation of EPA in TAG, such as EPA content in membranes at the start of nitrogen starvation, de novo synthesis and translocation, will be discussed.

The amount of EPA in polar lipids could be a limiting factor determining translocation into TAG. The lowest biomass-specific photon absorption rate had, however, the highest EPA and polar lipid content, but the lowest amount of EPA present in TAG. Suggesting, that the EPA accumulation in TAG is not limited by the amount of EPA present in the polar lipids. The culture with lowest biomass-specific photon supply rate has the lowest TAG content. Therefore, EPA accumulation in TAG might be correlated to TAG content and productivity.

Another hypothesis, for limited EPA accumulation in TAG could be the maximal amount of EPA that can accumulate in TAG without steric hindrance. In an engineered yeast Yarrowia lipolytica EPA accumulation up to $55 \%$ of total fatty acids in TAG was achieved (Xue et al., 2013), while in our work the maximum was $42 \%$ in TAG. Therefore, it is not likely that this limited the amount of EPA accumulated in TAG. In Trachydiscus minutus, a species from the same class as Nannochloropsis, also

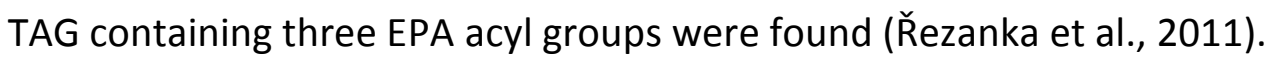

The translocation of EPA might be limited by the enzymes involved in translocation. Two type of enzymes were suggested to be involved in transport of fatty acids from membranes to TAG; phospholipid: diacylglycerol transferase (PDAT) and lipases. Transcriptomic analyses showed upregulation of one of the PDAT and several lipases (Chapter 3). Knock-out studies of these enzymes can provide more insight in their effect on the EPA accumulation in TAG during nitrogen starvation and allow specifying the enzyme(s) which are most important for translocation of EPA. Overexpression of these enzymes might increase translocation of EPA into TAG. Measuring expression levels of PDAT and lipases under conditions with increased or decreased EPA accumulation in TAG might also give indication on the specific genes responsible for translocation.

Furthermore, it would be interesting to study the substrate specificity of for example acyltransferases, since EPA seems to be translocated with higher affinity compared to other fatty acids (Chapter 2).

Finally, de novo synthesis can be rate limiting for EPA accumulation in TAG. The enzymes responsible for de novo EPA synthesis (e.g. elongases and desaturases) 
were mostly downregulated and might be rate-limiting in this process. Since oleic acid accumulation increased during nitrogen starvation, the elongases and desaturases used in the following steps of EPA synthesis are interesting targets for overexpression. If overexpression increases de novo synthesis of EPA, measuring the localization of EPA in TAG or polar lipids will help understand EPA accumulation in TAG during nitrogen starvation.

\subsection{CONCLUSIONS}

Most light regimes and biomass-specific photon supply rates at the onset of nitrogen starvation result in similar high TAG contents. The EPA content was highest at lowest biomass-specific photon supply rate mainly due to its presence in the polar lipids which are mostly located in the membranes. During nitrogen starvation, EPA accumulation in TAG was lower at the low biomass-specific photon supply rates which had the highest EPA content in polar lipids. This suggests that the amount of EPA in polar lipids is not limiting for the EPA accumulation in TAG. More research is necessary to understand the mechanisms and regulations involved in EPA accumulation during nitrogen starvation and its localization in the polar lipids and TAG. It is suggested to study the function of EPA in TAG using a nitrogen recovery experiment using labelled carbon $\left({ }^{13} \mathrm{C}\right)$. One of the possibilities to increase the EPA accumulation during nitrogen starvation is increase the amount accumulating in TAG. Knock out studies of PDAT and lipases genes can indicate which enzyme(s) are responsible for the translocation of EPA. These genes are possible targets for overexpression to increase the translocation of EPA into TAG. Next to increased translocation, de novo synthesis could be increased by the overexpression of elongases and desaturases involved in the EPA synthesis pathway. This shows that there are several future opportunities to study and increase EPA accumulation. 


\subsection{SUPPLEMENTS}

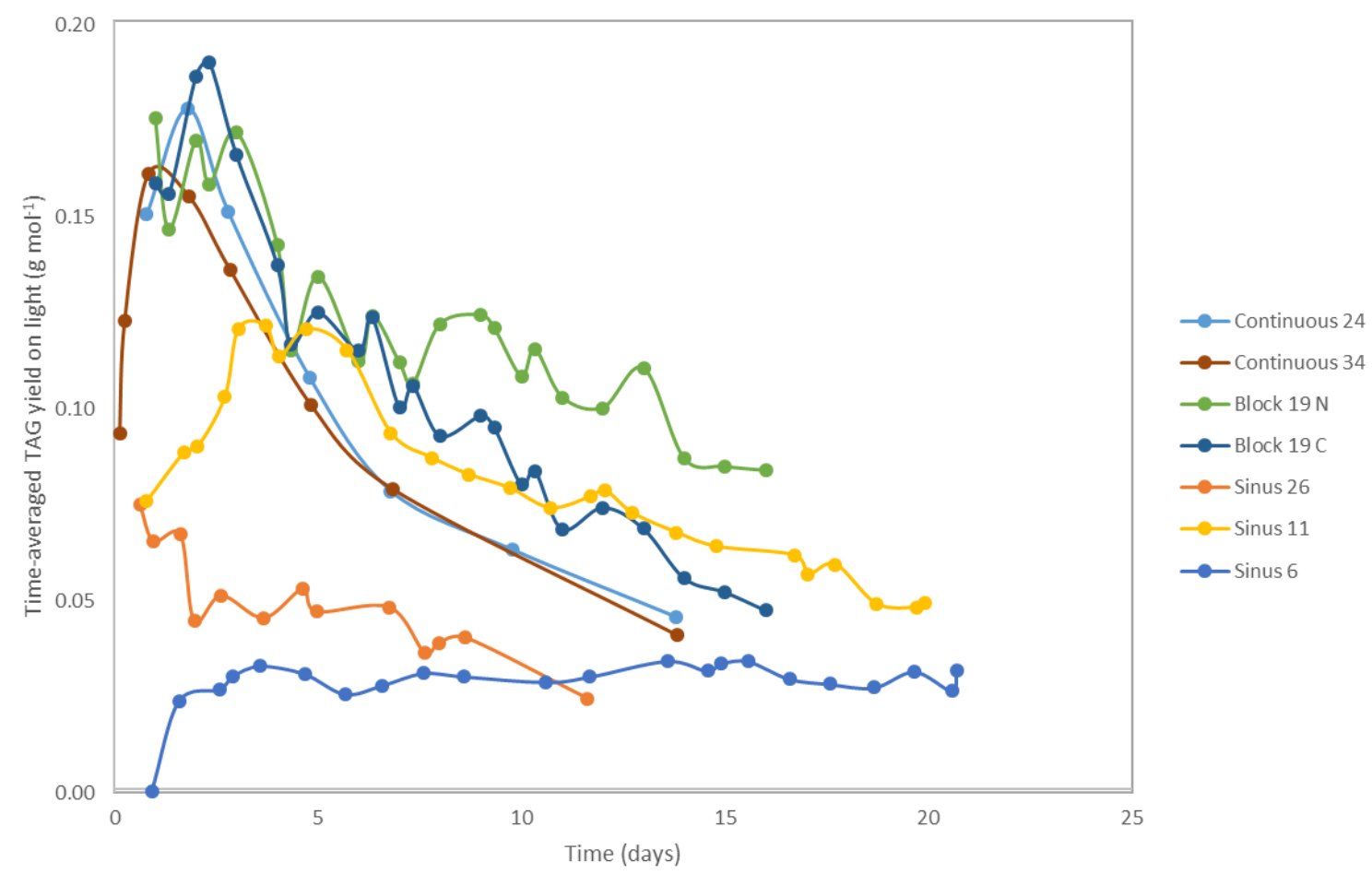

Figure S6.6. Time-averaged TAG yield on light $\left(\mathrm{g}_{\mathrm{TAG}} \mathrm{mol}_{\mathrm{ph}}{ }^{-1}\right)$ for the different biomass-specific photon supply rates and light regimes from the moment of nitrogen starvation. 


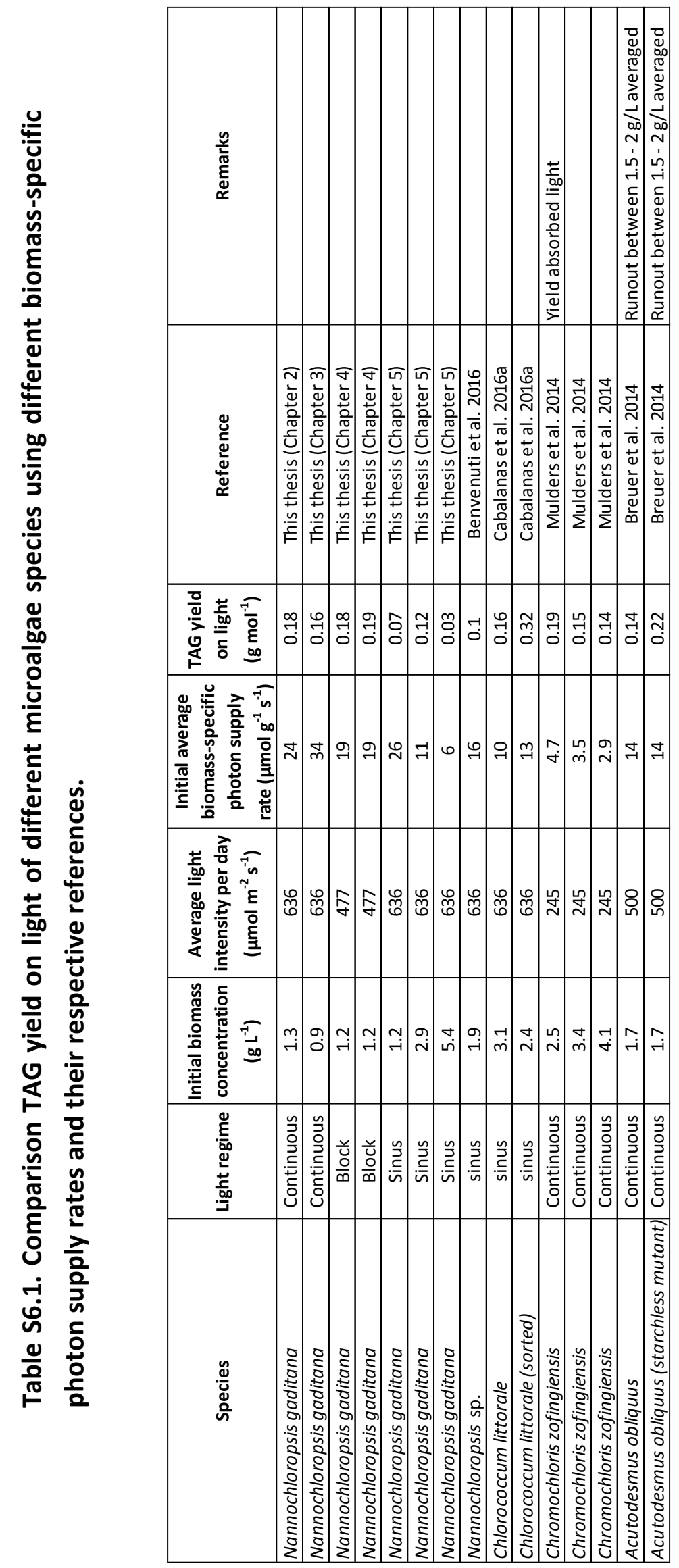




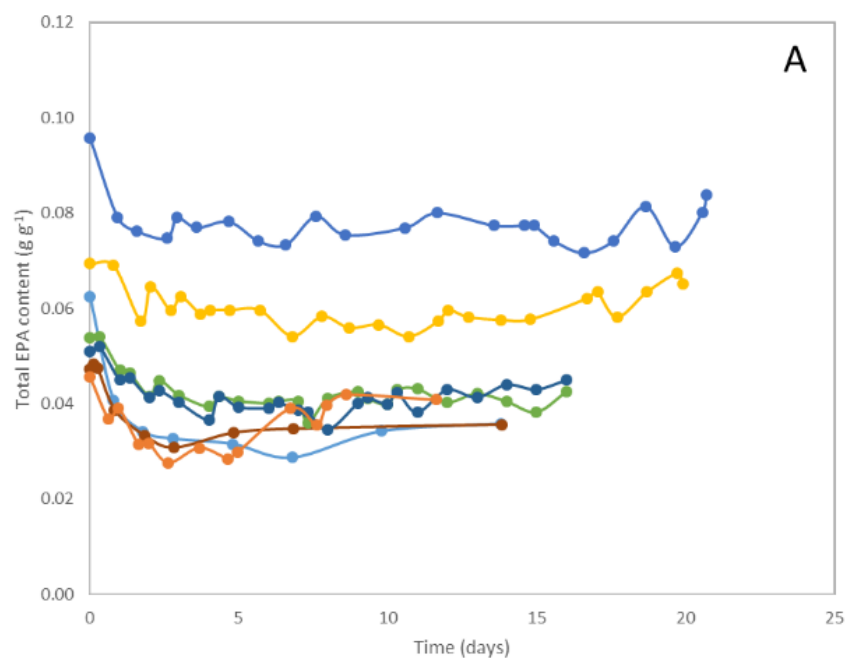

- Continuous 24 $\rightarrow$ Continuous 34 $\rightarrow$ Block $19 \mathrm{~N}$ $\rightarrow$ Block $19 \mathrm{C}$ $\rightarrow$ Sinus 26 $\rightarrow-$ Sinus 11 $\rightarrow$ Sinus 6
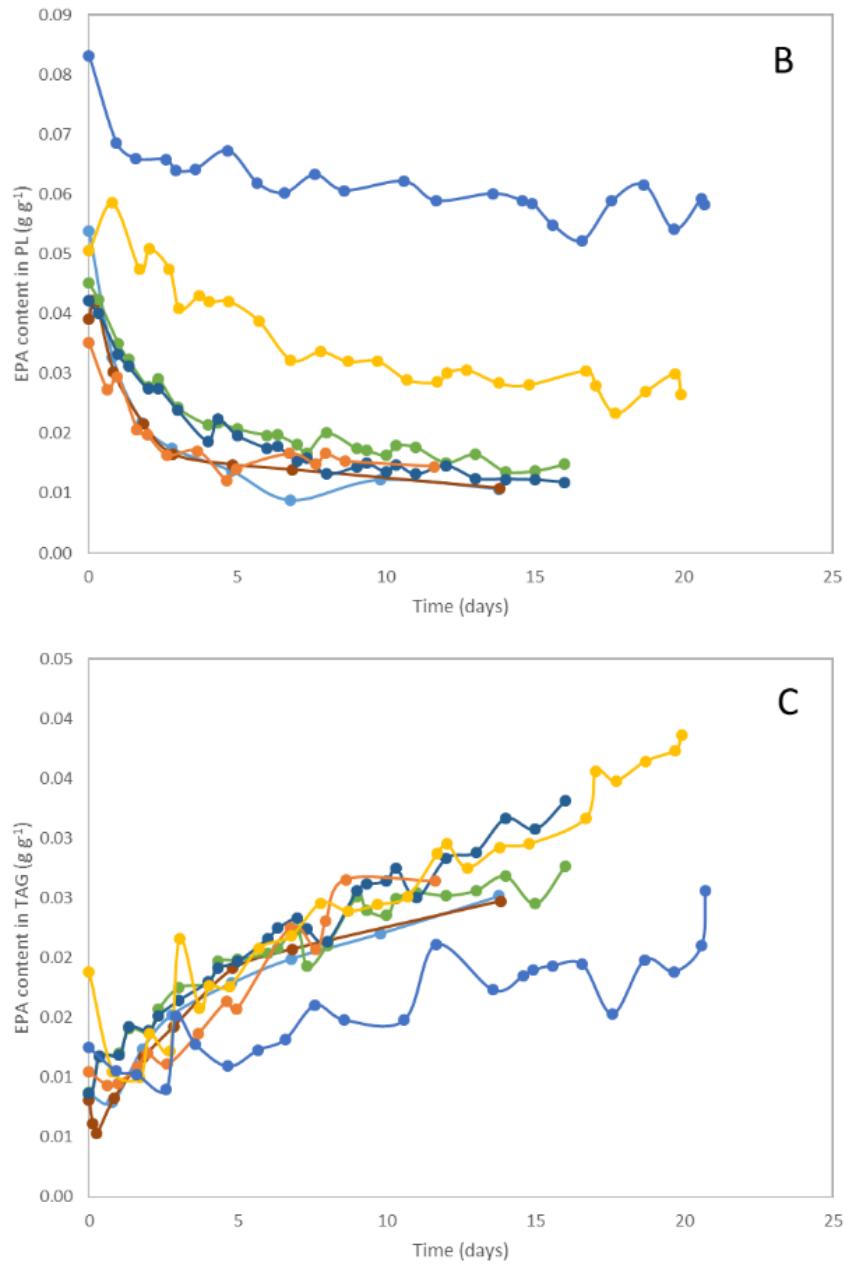

Figure S6.7. EPA content expressed per biomass $\left(g_{E P A} g_{d w}{ }^{-1}\right)$ in total lipids (TAG + PL) (A), in TAG (B) and polar lipids (PL) (C) for the different biomass-specific photon supply rates and light regimes from the moment of nitrogen starvation. 


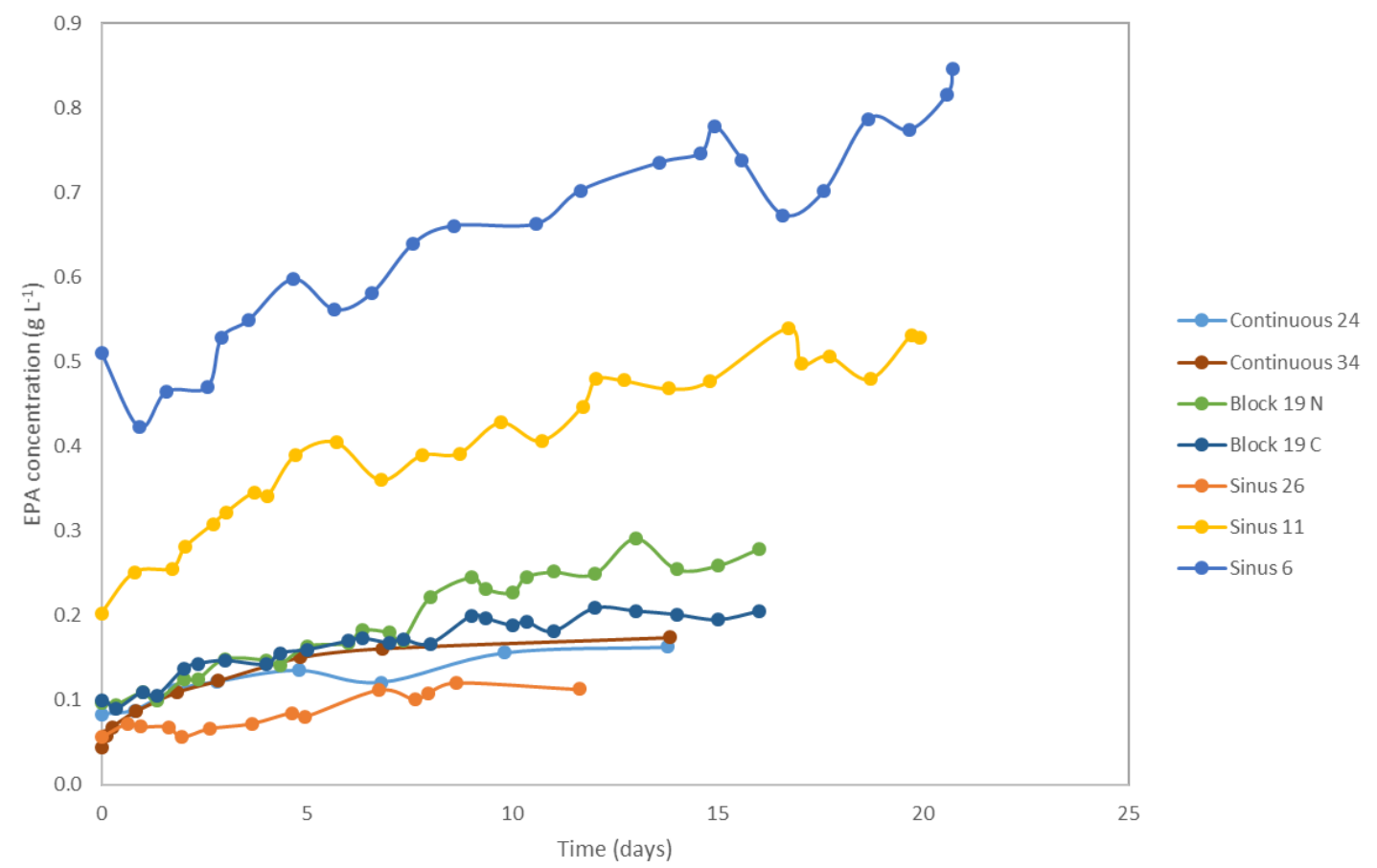

Figure S6.8. Total EPA concentration $\left(\mathrm{g} \mathrm{L}^{-1}\right)$ measured in the photobioreactor for the different biomass-specific photon supply rates and light regimes from the moment of nitrogen starvation. 


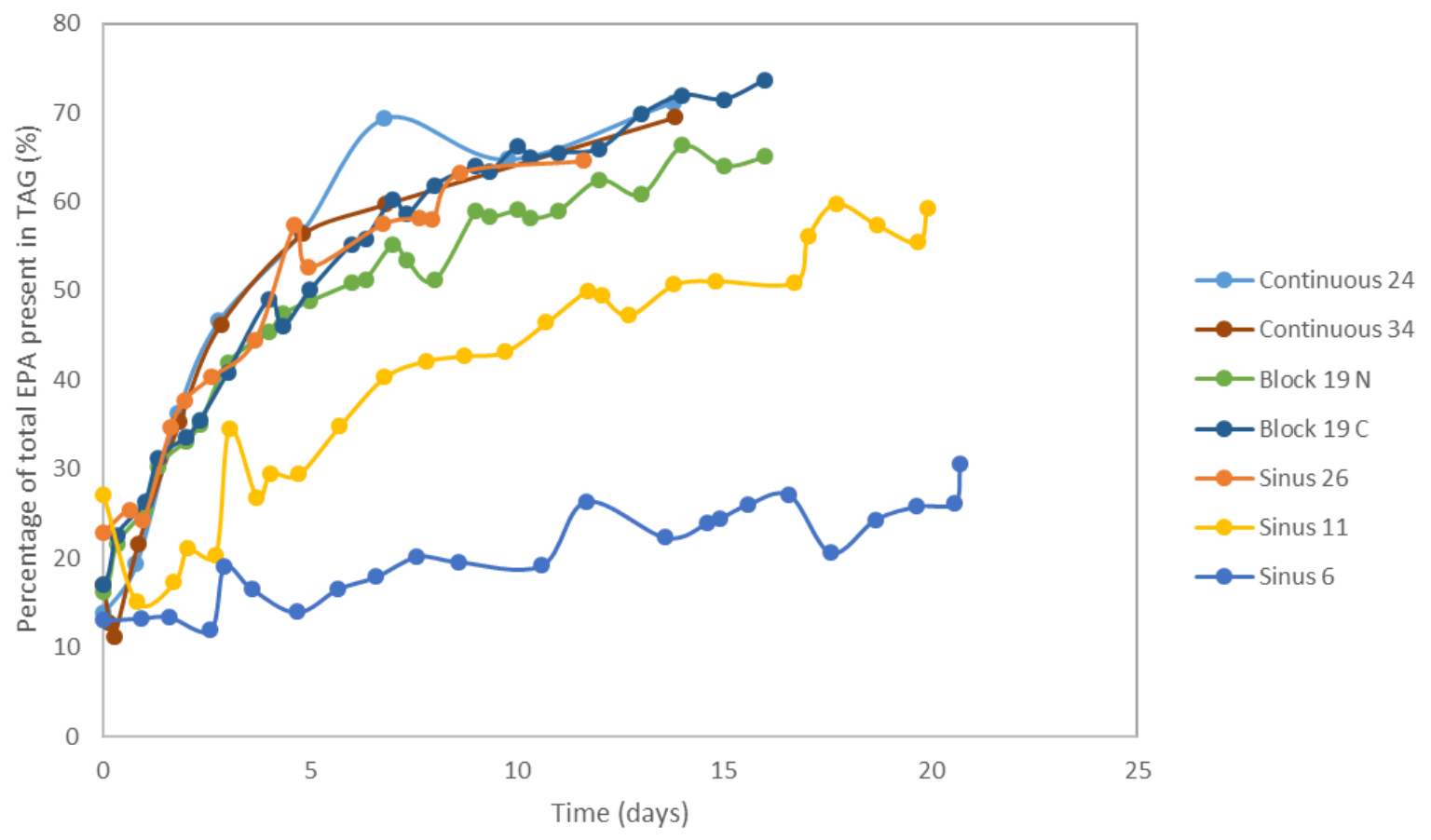

Figure S6.9. The percentage of all EPA in the cell which is present in TAG lipid fraction for the different biomass-specific photon supply rates and light regimes 


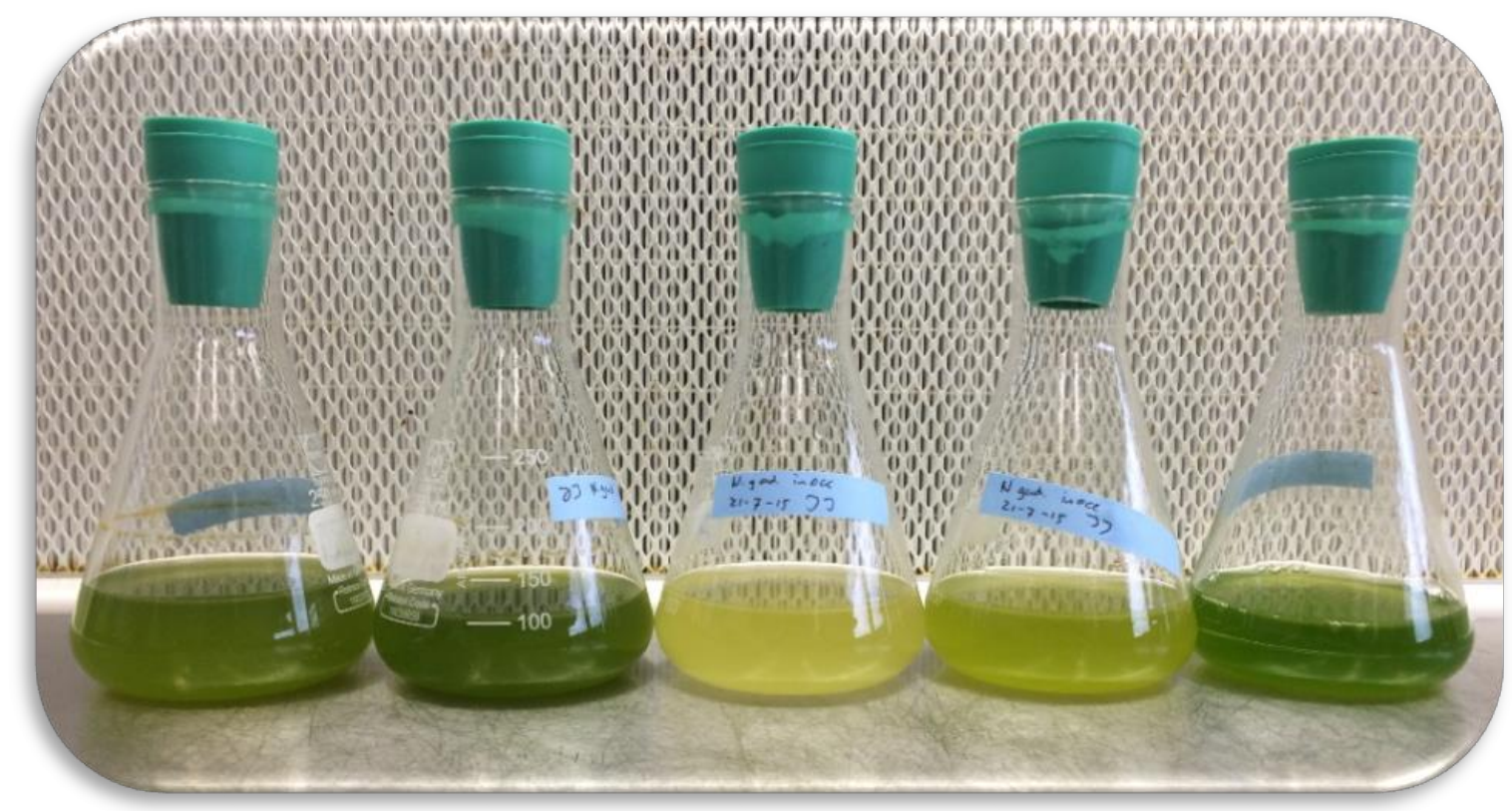

Colour change of Nannochloropsis in Erlenmeyer flasks when grown under different growth conditions. 
References

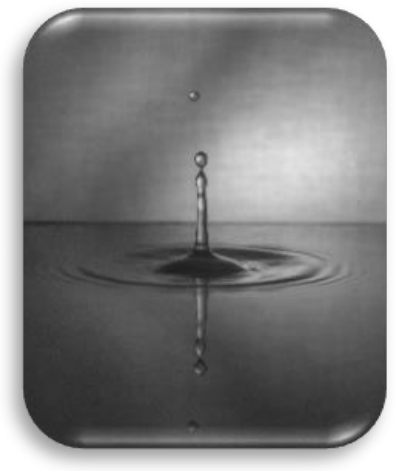




\section{REFERENCES}

Ajjawi, I., Verruto, J., Aqui, M., Soriaga, L.B., Coppersmith, J., Kwok, K., Peach, L., Orchard, E., Kalb, R., Xu, W., Carlson, T.J., Francis, K., Konigsfeld, K., Bartalis, J., Schultz, A., Lambert, W., Schwartz, A.S., Brown, R., Moellering, E.R., 2017. Lipid production in Nannochloropsis gaditana is doubled by decreasing expression of a single transcriptional regulator. Nat. Biotechnol. 35, 647-652. doi:10.1038/nbt.3865

Alboresi, A., Perin, G., Vitulo, N., Diretto, G., Block, M.A., Jouhet, J., Meneghesso, A., Valle, G., Giuliano, G., Maréchal, E., Morosinotto, T., 2016. Light Remodels Lipid Biosynthesis in Nannochloropsis gaditana by Modulating Carbon Partitioning Between Organelles. Plant Physiol. 171, 2468-2482. doi:10.1104/pp.16.00599

Banerjee, A., Maiti, S.K., Guria, C., Banerjee, C., 2017. Metabolic pathways for lipid synthesis under nitrogen stress in Chlamydomonas and Nannochloropsis. Biotechnol. Lett. 39, 111. doi:10.1007/s10529-016-2216-y

Benvenuti, G., Bosma, R., Cuaresma, M., Janssen, M., Barbosa, M.J., Wijffels, R.H., 2015. Selecting microalgae with high lipid productivity and photosynthetic activity under nitrogen starvation. J. Appl. Phycol. 27, 1425-1431. doi:10.1007/s10811-014-0470-8

Benvenuti, G., Bosma, R., Ji, F., Lamers, P., Barbosa, M.J., Wijffels, R.H., 2016a. Batch and semicontinuous microalgal TAG production in lab-scale and outdoor photobioreactors. J. Appl. Phycol. 28, 3167-3177. doi:10.1007/s10811-016-0897-1

Benvenuti, G., Lamers, P.P., Breuer, G., Bosma, R., Cerar, A., Wijffels, R.H., Barbosa, M.J., 2016b. Microalgal TAG production strategies: why batch beats repeated-batch. Biotechnol. Biofuels 9, 64. doi:10.1186/s13068-016-0475-4

Boyle, N.R., Page, M.D., Liu, B., Blaby, I.K., Casero, D., Kropat, J., Cokus, S.J., Hong-Hermesdorf, A., Shaw, J., Karpowicz, S.J., Gallaher, S.D., Johnson, S., Benning, C., Pellegrini, M., Grossman, A., Merchant, S.S., 2012. Three acyltransferases and nitrogen-responsive regulator are implicated in nitrogen starvation-induced triacylglycerol accumulation in Chlamydomonas. J. Biol. Chem. 287, 15811-15825. doi:10.1074/jbc.M111.334052

Breuer, G., De Jaeger, L., Artus, V.P.G., Martens, D.E., Springer, J., Draaisma, R.B., Eggink, G., Wijffels, R.H., Lamers, P.P., 2014. Superior triacylglycerol (TAG) accumulation in starchless mutants of Scenedesmus obliquus: (II) evaluation of TAG yield and productivity in controlled photobioreactor. Biotechnol. Biofuels 7. doi:10.1186/17546834-7-70

Breuer, G., Lamers, P.P., Martens, D.E., Draaisma, R.B., Wijffels, R.H., 2012. The impact of nitrogen starvation on the dynamics of triacylglycerol accumulation in nine microalgae strains. Bioresour. Technol. 124, 217-226. doi:10.1016/j.biortech.2012.08.003

Bromke, M.A., Hochmuth, A., Tohge, T., Fernie, A.R., Giavalisco, P., Burgos, A., Willmitzer, L., Brotman, Y., 2015. Liquid chromatography high-resolution mass spectrometry for fatty acid profiling. Plant J. 81, 529-536. doi:10.1111/tpj.12739 
Cabanelas, I.T.D., Van Der Zwart, M., Kleinegris, D.M.M., Wijffels, R.H., Barbosa, M.J., 2016. Sorting cells of the microalga Chlorococcum littorale with increased triacylglycerol productivity. Biotechnol. Biofuels 9, 1-12. doi:10.1186/s13068-016-0595-x

Camacho-Rodríguez, J., González-Céspedes, A.M., Cerón-García, M.C., Fernández-Sevilla, J.M., Acién-Fernández, F.G., Molina-Grima, E., 2014. A quantitative study of eicosapentaenoic acid (EPA) production by Nannochloropsis gaditana for aquaculture as a function of dilution rate, temperature and average irradiance. Appl. Microbiol. Biotechnol. 98, 2429-2440. doi:10.1007/s00253-013-5413-9

Chapman, R.L., 2013. Algae: The world's most important "plants"-an introduction. Mitig. Adapt. Strateg. Glob. Chang. 18, 5-12. doi:10.1007/s11027-010-9255-9

Chisti, Y., 2007. Biodiesel from microalgae. Biotechnol. Adv. 25, 294-306. doi:10.1016/j.biotechadv.2007.02.001

Cohen, Z., Khozin-Goldberg, I., Adlerstein, D., Bigogno, C., 2000. The role of triacylglycerol as a reservoir of polyunsaturated fatty acids for the rapid production of chloroplastic lipids in certain microalgae. Biochem. Soceity Trans. 28, 740-743.

Corteggiani Carpinelli, E., Telatin, A., Vitulo, N., Forcato, C., D’Angelo, M., Schiavon, R., Vezzi, A., Giacometti, G.M., Morosinotto, T., Valle, G., 2014. Chromosome Scale Genome Assembly and Transcriptome Profiling of Nannochloropsis gaditana in Nitrogen Depletion. Mol. Plant 7, 323-335. doi:10.1093/mp/sst120

Dam, J.C.J. Van, Koehorst, J.J., Vik, J.O., Schaap, P.J., Suarez-diez, M., 2017. Interoperable genome annotation with $\mathrm{GBOL}$, an extendable infrastructure for functional data mining. bioRxiv 1-9. doi:10.1101/184747

de Jaeger, L., Springer, J., Wolbert, E.J.H., Martens, D.E., Eggink, G., Wijffels, R.H., 2017. Gene silencing of stearoyl-ACP desaturase enhances the stearic acid content in Chlamydomonas reinhardtii. Bioresour. Technol. 245, 1616-1626. doi:10.1016/j.biortech.2017.06.128

de Mooij, T., Janssen, M., Cerezo-Chinarro, O., Mussgnug, J.H., Kruse, O., Ballottari, M., Bassi, R., Bujaldon, S., Wollman, F.A., Wijffels, R.H., 2015. Antenna size reduction as a strategy to increase biomass productivity: A great potential not yet realized. J. Appl. Phycol. 27, 1063-1077. doi:10.1007/s10811-014-0427-y

Dobin, A., Davis, C.A., Schlesinger, F., Drenkow, J., Zaleski, C., Jha, S., Batut, P., Chaisson, M., Gingeras, T.R., 2013. STAR: Ultrafast universal RNA-seq aligner. Bioinformatics 29, 1521. doi:10.1093/bioinformatics/bts635

Dolch, L.-J., Rak, C., Perin, G., Tourcier, G., Broughton, R., Leterrier, M., Morosinotto, T., Tellier, F., Faure, J.-D., Falconet, D., Jouhet, J., Sayanova, O., Beaudoin, F., Maréchal, E., 2017. A Palmitic Acid Elongase Affects Eicosapentaenoic Acid and Plastidial Monogalactosyldiacylglycerol Levels in Nannochloropsis. Plant Physiol. 173, 742-759. doi:10.1104/pp.16.01420 
Dong, H.-P., Williams, E., Wang, D., Xie, Z.-X., Hsia, R., Jenck, A., Halden, R., Li, J., Chen, F., Place, A.R., 2013. Responses of Nannochloropsis oceanica IMET1 to Long-Term Nitrogen Starvation and Recovery. Plant Physiol. 162, 1110-26. doi:10.1104/pp.113.214320

Draaisma, R.B., Wijffels, R.H., Slegers, P.M., Brentner, L.B., Roy, A., Barbosa, M.J., 2013. Food commodities from microalgae. Curr. Opin. Biotechnol. 24, 169-177. doi:10.1016/j.copbio.2012.09.012

Dubois, M., Gilles, K.A., Hamilton, J.K., Rebers, P.A., Smith, F., 1956. Colorimetric Method for Determination of Sugars and Related Substances. Anal. Chem. 28, 350-356. doi:10.1021/ac60111a017

Falkowski, P.G., Owens, T.G., 1980. Light-Shade Adaptation - two strategies in marine phytoplankton. Plant Physiol. 66, 592-595. doi:10.1104/pp.66.4.592

Fawley, M.W., Jameson, I., Fawley, K.P., 2015. The phylogeny of the genus Nannochloropsis (Monodopsidaceae, Eustigmatophyceae), with descriptions of $N$. australis sp. nov . and Microchloropsis gen. nov . Phycologia 54, 545-552. doi:10.2216/15-60.1

Feng, P., Deng, Z., Hu, Z., Fan, L., 2011. Lipid accumulation and growth of Chlorella zofingiensis in flat plate photobioreactors outdoors. Bioresour. Technol. 102, 10577-10584. doi:10.1016/j.biortech.2011.08.109

Flynn, K.J., Davidson, K., Cunningham, A., 1993. Relations between carbon and nitrogen during growth of Nannochloropsis oculata (Droop) Hibberd under continuous illumination. New Phytol. 125, 717-722.

Godfray, H.C.J., Beddington, J.R., Crute, I.R., Haddad, L., Lawrence, D., Muir, J.F., Pretty, J., Robinson, S., Thomas, S.M., Toulmin, C., 2010. Food security: The challenge of feeding 9 billion people. Science (80-. ). 327, 812-818. doi:10.1126/science.1185383

Gordon, J.M., Polle, J.E.W., 2007. Ultrahigh bioproductivity from algae. Appl. Microbiol. Biotechnol. 76, 969-975. doi:10.1007/s00253-007-1102-x

Guihéneuf, F., Stengel, D.B., 2013. LC-PUFA-enriched oil production by microalgae: Accumulation of lipid and triacylglycerols containing n-3 LC-PUFA is triggered by nitrogen limitation and inorganic carbon availability in the marine haptophyte Pavlova lutheri. Mar. Drugs 11, 4246-4266. doi:10.3390/md11114246

Guiry, M.D., 2012. How many species of algae are there? J. Phycol. 48, 1057-1063. doi:10.1111/j.1529-8817.2012.01222.x

Han, B., Virtanen, M., Koponen, J., Straškraba, M., 2000. Effect of photoinhibition on algal photosynthesis : a dynamic model. J. Plankton Res. 22, 865-885.

Hooft, J.J.J. van der, Vervoort, J., Bino, R.J., Vos, R.C.H. de, 2012. Spectral trees as a robust annotation tool in LC-MS based metabolomics. Metabolomics 8, 691-703. doi:10.1007/s11306-011-0363-7 
Hu, Q., Sommerfeld, M., Jarvis, E., Ghirardi, M., Posewitz, M., Seibert, M., Darzins, A., 2008. Microalgal triacylglycerols as feedstocks for biofuel production: perspectives and advances. Plant J. 54, 621-639. doi:10.1111/j.1365-313X.2008.03492.x

Hulatt, C.J., Wijffels, R.H., Bolla, S., Kiron, V., 2017. Production of fatty acids and protein by Nannochloropsis in flat-plate photobioreactors. PLoS One 12, 1-17. doi:10.1371/journal.pone.0170440

Iwai, M., Hori, K., Sasaki-Sekimoto, Y., Shimojima, M., Ohta, H., 2015. Manipulation of oil synthesis in Nannochloropsis strain NIES-2145 with a phosphorus starvation-inducible promoter from Chlamydomonas reinhardtii. Front. Microbiol. 6. doi:10.3389/fmicb.2015.00912

Jeanfils, J., Canisius, M.-F., Burlion, N., 1993. Effect of high nitrate concentrations on growth and nitrate uptake by free-living and immobilized Chlorella vulgaris cells. J. Appl. Phycol. 5, 369-374. doi:10.1007/BF02186240

Jia, J., Han, D., Gerken, H.G., Li, Y., Sommerfeld, M., Hu, Q., Xu, J., 2015. Molecular mechanisms for photosynthetic carbon partitioning into storage neutral lipids in Nannochloropsis oceanica under nitrogen-depletion conditions. Algal Res. 7, 66-77. doi:10.1016/j.algal.2014.11.005

Kandilian, R., Pruvost, J., Legrand, J., Pilon, L., 2014. Influence of light absorption rate by Nannochloropsis oculata on triglyceride production during nitrogen starvation. Bioresour. Technol. 163, 308-319. doi:10.1016/j.biortech.2014.04.045

Kaye, Y., Grundman, O., Leu, S., Zarka, A., Zorin, B., Didi-Cohen, S., Khozin-Goldberg, I., Boussiba, S., 2015. Metabolic engineering toward enhanced LC-PUFA biosynthesis in Nannochloropsis oceanica: Overexpression of endogenous $\delta 12$ desaturase driven by stress-inducible promoter leads to enhanced deposition of polyunsaturated fatty acids in TAG. Algal Res. 11, 387-398. doi:10.1016/j.algal.2015.05.003

Khozin-Goldberg, I., Cohen, Z., 2011. Unraveling algal lipid metabolism: Recent advances in gene identification. Biochimie 93, 91-100. doi:10.1016/j.biochi.2010.07.020

Khozin-Goldberg, I., Shrestha, P., Cohen, Z., 2005. Mobilization of arachidonyl moieties from triacylglycerols into chloroplastic lipids following recovery from nitrogen starvation of the microalga Parietochloris incisa. Biochim. Biophys. Acta 1738, 63-71. doi:10.1016/j.bbalip.2005.09.005

Kilian, O., Benemann, C.S.E., Niyogi, K.K., Vick, B., 2011. High-efficiency homologous recombination in the oil-producing alga Nannochloropsis sp. Proc. Natl. Acad. Sci. 108, 21265-21269. doi:10.1073/pnas.1105861108

Kliphuis, A.M.J., de Winter, L., Vejrazka, C., Martens, D.E., Janssen, M., Wijffels, R.H., 2010. Photosynthetic efficiency of Chlorella sorokiniana in a turbulently mixed short lightpath photobioreactor. Biotechnol. Prog. 26, 687-696. doi:10.1002/btpr.379 
Kliphuis, A.M.J., Klok, A.J., Martens, D.E., Lamers, P.P., Janssen, M., Wijffels, R.H., 2012. Metabolic modeling of Chlamydomonas reinhardtii: Energy requirements for photoautotrophic growth and maintenance. J. Appl. Phycol. 24, 253-266. doi:10.1007/s10811-011-9674-3

Klok, A.J., Martens, D.E., Wijffels, R.H., Lamers, P.P., 2013. Simultaneous growth and neutral lipid accumulation in microalgae. Bioresour. Technol. 134, 233-243. doi:10.1016/j.biortech.2013.02.006

Koehorst, J.J., van Dam, J.C.J., Saccenti, E., Martins dos Santos, V.A.P., Suarez-Diez, M., Schaap, P.J., 2017. SAPP: functional genome annotation and analysis through a semantic framework using FAIR principles. Bioinformatics 1-3. doi:10.1093/bioinformatics/btx767

Lamers, P.P., Van De Laak, C.C.W., Kaasenbrood, P.S., Lorier, J., Janssen, M., De Vos, R.C.H., Bino, R.J., Wijffels, R.H., 2010. Carotenoid and fatty acid metabolism in light-stressed Dunaliella salina. Biotechnol. Bioeng. 106, 638-648. doi:10.1002/bit.22725

León-Saiki, G.M., Remmers, I.M., Martens, D.E., Lamers, P.P., Wijffels, R.H., van der Veen, D., 2017. The role of starch as transient energy buffer in synchronized microalgal growth in Acutodesmus obliquus. Algal Res. 25, 160-167. doi:10.1016/j.algal.2017.05.018

Li, J., Han, D., Wang, D., Ning, K., Jia, J., Wei, L., Jing, X., Huang, S., Chen, J., Li, Y., Hu, Q., Xu, J., 2014. Choreography of Transcriptomes and Lipidomes of Nannochloropsis Reveals the Mechanisms of Oil Synthesis in Microalgae. Plant Cell 26, 1645-1665. doi:10.1105/tpc.113.121418

Li, X., Moellering, E.R., Liu, B., Johnny, C., Fedewa, M., Sears, B.B., Kuo, M.-H., Benning, C., 2012. A Galactoglycerolipid Lipase Is Required for Triacylglycerol Accumulation and Survival Following Nitrogen Deprivation in Chlamydomonas reinhardtii. Plant Cell 24, 4670-4686. doi:10.1105/tpc.112.105106

Li, Y., Fei, X., Deng, X., 2012. Novel molecular insights into nitrogen starvation-induced triacylglycerols accumulation revealed by differential gene expression analysis in green algae Micractinium pusillum. Biomass and Bioenergy 42, 199-211. doi:10.1016/j.biombioe.2012.03.010

Liu, B., Benning, C., 2013. Lipid metabolism in microalgae distinguishes itself. Curr. Opin. Biotechnol. 24, 300-309. doi:10.1016/j.copbio.2012.08.008

Lommen, A., Kools, H.J., 2012. MetAlign 3.0: Performance enhancement by efficient use of advances in computer hardware. Metabolomics 8, 719-726. doi:10.1007/s11306-0110369-1

Love, M.I., Huber, W., Anders, S., 2014. Moderated estimation of fold change and dispersion for RNA-seq data with DESeq2. Genome Biol. 15, 1-21. doi:10.1186/s13059-014-05508 
Lubián, L.M., 1982. Nannochloropsis gaditana sp. no, unv., una nueva Eustiginatophyeeae marina. Lazaroa 4, 287-293. doi:-

Ma, X., Yu, J., Zhu, B., Pan, K., Pan, J., Yang, G., 2011. Cloning and characterization of a delta-6 desaturase encoding gene from Nannochloropsis oculata. Chinese J. Oceanol. Limnol. 29, 290-296. doi:10.1007/s00343-011-0048-0

Ma, X.N., Chen, T.-P., Yang, B., Liu, J., Chen, F., 2016. Lipid production from Nannochloropsis. Mar. Drugs 14. doi:10.3390/md14040061

Meng, Y., Jiang, J., Wang, H., Cao, X., Xue, S., Yang, Q., Wang, W., 2015. The characteristics of TAG and EPA accumulation in Nannochloropsis oceanica IMET1 under different nitrogen supply regimes. Bioresour. Technol. 179, 483-489. doi:10.1016/j.biortech.2014.12.012

Miller, R., Wu, G., Deshpande, R.R., Vieler, A., Gartner, K., Li, X., Moellering, E.R., Zauner, S., Cornish, A.J., Liu, B., Bullard, B., Sears, B.B., Kuo, M.-H., Hegg, E.L., Shachar-Hill, Y., Shiu, S.-H., Benning, C., 2010. Changes in Transcript Abundance in Chlamydomonas reinhardtii following Nitrogen Deprivation Predict Diversion of Metabolism. Plant Physiol. 154, 1737-1752. doi:10.1104/pp.110.165159

Mooij, P.R., de Graaff, D.R., van Loosdrecht, M.C.M., Kleerebezem, R., 2015. Starch productivity in cyclically operated photobioreactors with marine microalgae-effect of ammonium addition regime and volume exchange ratio. J. Appl. Phycol. 27, 1121-1126. doi:10.1007/s10811-014-0430-3

Mühlroth, A., Li, K., Røkke, G., Winge, P., Olsen, Y., Hohmann-Marriott, M.F., Vadstein, O., Bones, A.M., 2013. Pathways of lipid metabolism in marine algae, co-expression network, bottlenecks and candidate genes for enhanced production of EPA and DHA in species of chromista. Mar. Drugs 11, 4662-4697. doi:10.3390/md11114662

Mulders, K.J.M., Janssen, J.H., Martens, D.E., Wijffels, R.H., Lamers, P.P., 2014a. Effect of biomass concentration on secondary carotenoids and triacylglycerol (TAG) accumulation in nitrogen-depleted Chlorella zofingiensis. Algal Res. 6, 8-16. doi:10.1016/j.algal.2014.08.006

Mulders, K.J.M., Lamers, P.P., Wijffels, R.H., Martens, D.E., 2014b. Dynamics of biomass composition and growth during recovery of nitrogen-starved Chromochloris zofingiensis. Appl. Microbiol. Biotechnol. 99, 1873-1884. doi:10.1007/s00253-014$6181-x$

Mus, F., Toussaint, J.-P., Cooksey, K.E., Fields, M.W., Gerlach, R., Peyton, B.M., Carlson, R.P., 2013. Physiological and molecular analysis of carbon source supplementation and $\mathrm{pH}$ stress-induced lipid accumulation in the marine diatom Phaeodactylum tricornutum. Appl. Microbiol. Biotechnol. 97, 3625-3642. doi:10.1007/s00253-013-4747-7

Nikolaou, A., Hartmann, P., Sciandra, A., Chachuat, B., Bernard, O., 2016. Dynamic coupling of photoacclimation and photoinhibition in a model of microalgae growth. J. Theor. Biol. 390, 61-72. doi:10.1016/j.jtbi.2015.11.004 
Pal, D., Khozin-Goldberg, I., Cohen, Z., Boussiba, S., 2011. The effect of light, salinity, and nitrogen availability on lipid production by Nannochloropsis sp. Appl. Microbiol. Biotechnol. 90, 1429-1441. doi:10.1007/s00253-011-3170-1

Peng, K.-T., Zheng, C.-N., Xue, J., Chen, X.-Y., Yang, W.-D., Liu, J.-S., Bai, W., Li, H.-Y., 2014. Delta 5 Fatty Acid Desaturase Upregulates the Synthesis of Polyunsaturated Fatty Acids in the Marine Diatom Phaeodactylum tricornutum. J. Agric. Food Chem. 62, 8773-8776. doi:10.1021/jf5031086

Postma, P.R., Miron, T.L., Olivieri, G., Barbosa, M.J., Wijffels, R.H., Eppink, M.H.M., 2015. Mild disintegration of the green microalgae Chlorella vulgaris using bead milling. Bioresour. Technol. 184, 297-304. doi:10.1016/j.biortech.2014.09.033

Radakovits, R., Jinkerson, R.E., Fuerstenberg, S.I., Tae, H., Settlage, R.E., Boore, J.L., Posewitz, M.C., 2012. Draft genome sequence and genetic transformation of the oleaginous alga Nannochloropis gaditana. Nat. Commun. 3, 686. doi:10.1038/ncomms1688

Remmers, I.M., Hidalgo-Ulloa, A., Brandt, B.P., Evers, W.A.C., Wijffels, R.H., Lamers, P.P., 2017a. Continuous versus batch production of lipids in the microalgae Acutodesmus obliquus. Bioresour. Technol. 244, 1384-1392. doi:10.1016/j.biortech.2017.04.093

Remmers, I.M., Martens, D.E., Wijffels, R.H., Lamers, P.P., 2017b. Dynamics of triacylglycerol and EPA production in Phaeodactylum tricornutum under nitrogen starvation at different light intensities. PLoS One 12, 1-13. doi:10.1371/journal.pone.0175630

Řezanka, T., Lukavský, J., Nedbalová, L., Sigler, K., 2011. Effect of nitrogen and phosphorus starvation on the polyunsaturated triacylglycerol composition, including positional isomer distribution, in the alga Trachydiscus minutus. Phytochemistry 72, 2342-2351. doi:10.1016/j.phytochem.2011.08.017

Rismani-Yazdi, H., Haznedaroglu, B.Z., Hsin, C., Peccia, J., 2012. Transcriptomic analysis of the oleaginous microalga Neochloris oleoabundans reveals metabolic insightsinto triacylglyceride accumulation. Biotechnol. Biofuels 5.

Rodolfi, L., Zittelli, G.C., Bassi, N., Padovani, G., Biondi, N., Bonini, G., Tredici, M.R., 2009. Microalgae for oil: Strain selection, induction of lipid synthesis and outdoor mass cultivation in a low-cost photobioreactor. Biotechnol. Bioeng. 102, 100-112. doi:10.1002/bit.22033

Rubio, F.C., Camacho, F.G., Sevilla, J.M.F., Chisti, Y., Grima, E.M., 2003. A mechanistic model of photosynthesis in microalgae. Biotechnol. Bioeng. 81, 459-473. doi:10.1002/bit.10492

Ruiz, J., Olivieri, G., de Vree, J., Bosma, R., Willems, P., Reith, J.H., Eppink, M.H.M., Kleinegris, D.M.M., Wijffels, R.H., Barbosa, M.J., 2016. Towards industrial products from microalgae. Energy Environ. Sci. 9, 3036-3043. doi:10.1039/C6EE01493C 
San Pedro, A., González-López, C. V., Acién, F.G., Molina-Grima, E., 2016. Outdoor pilot production of Nannochloropsis gaditana: Influence of culture parameters and lipid production rates in flat-panel photobioreactors. Algal Res. 18, 156-165. doi:10.1016/j.algal.2016.06.011

Sanz-Luque, E., Chamizo-Ampudia, A., Llamas, A., Galvan, A., Fernandez, E., 2015. Understanding nitrate assimilation and its regulation in microalgae. Front. Plant Sci. 6. doi:10.3389/fpls.2015.00899

Schneider, J.C., Roessler, P., 1994. Radiolabeling studies of lipids and fatty acids in Nannochloropsis (eustigmatophyceae), an oleaginous marine alga. J. Phycol. 30, 594598. doi:10.1111/j.0022-3646.1994.00594.x

Schüler, L.M., Schulze, P.S.C., Pereira, H., Barreira, L., León, R., Varela, J., 2017. Trends and strategies to enhance triacylglycerols and high-value compounds in microalgae. Algal Res. 25, 263-273. doi:10.1016/j.algal.2017.05.025

Siaut, M., Cuiné, S., Cagnon, C., Fessler, B., Nguyen, M., Carrier, P., Beyly, A., Beisson, F., Triantaphylidès, C., Li-Beisson, Y., Peltier, G., 2011. Oil accumulation in the model green alga Chlamydomonas reinhardtii: characterization, variability between common laboratory strains and relationship with starch reserves. BMC Biotechnol. 11, 7. doi:10.1186/1472-6750-11-7

Simionato, D., Block, M.A., La Rocca, N., Jouhet, J., Maréchal, E., Finazzi, G., Morosinotto, T., 2013. The response of Nannochloropsis gaditana to nitrogen starvation includes de novo biosynthesis of triacylglycerols, a decrease of chloroplast galactolipids, and reorganization of the photosynthetic apparatus. Eukaryot. Cell 12, 665-676. doi:10.1128/EC.00363-12

Starkenburg, S.R., Kwon, K.J., Jha, R.K., McKay, C., Jacobs, M., Chertkov, O., Twary, S., Rocap, G., Cattolico, R.A., 2014. A pangenomic analysis of the Nannochloropsis organellar genomes reveals novel genetic variations in key metabolic genes. BMC Genomics 15, 1-21. doi:10.1186/1471-2164-15-212

Tonon, T., Harvey, D., Larson, T.R., Graham, I.A., 2002. Long chain polyunsaturated fatty acid production and partitioning to triacylglycerols in four microalgae. Phytochemistry 61, 15-24. doi:10.1016/S0031-9422(02)00201-7

Valledor, L., Furuhashi, T., Recuenco-Muñoz, L., Wienkoop, S., Weckwerth, W., 2014. Systemlevel network analysis of nitrogen starvation and recovery in Chlamydomonas reinhardtii reveals potential new targets for increased lipid accumulation. Biotechnol. Biofuels 7, 171. doi:10.1186/s13068-014-0171-1

Van Wagenen, J., Miller, T.W., Hobbs, S., Hook, P., Crowe, B., Huesemann, M., 2012. Effects of light and temperature on fatty acid production in Nannochloropsis salina. Energies 5, 731-740. doi:10.3390/en5030731 
Vieler, A., Wu, G., Tsai, C.H., Bullard, B., Cornish, A.J., Harvey, C., Reca, I.B., Thornburg, C., Achawanantakun, R., Buehl, C.J., Campbell, M.S., Cavalier, D., Childs, K.L., Clark, T.J., Deshpande, R., Erickson, E., Armenia Ferguson, A., Handee, W., Kong, Q., Li, X., Liu, B., Lundback, S., Peng, C., Roston, R.L., Sanjaya, Simpson, J.P., TerBush, A., Warakanont, J., Zäuner, S., Farre, E.M., Hegg, E.L., Jiang, N., Kuo, M.H., Lu, Y., Niyogi, K.K., Ohlrogge, J., Osteryoung, K.W., Shachar-Hill, Y., Sears, B.B., Sun, Y., Takahashi, H., Yandell, M., Shiu, S.H., Benning, C., 2012. Genome, Functional Gene Annotation, and Nuclear Transformation of the Heterokont Oleaginous Alga Nannochloropsis oceanica CCMP1779. PLoS Genet. 8. doi:10.1371/journal.pgen.1003064

Wang, D., Ning, K., Li, J., Hu, J., Han, D., Wang, H., Zeng, X., Jing, X., Zhou, Q., Su, X., Chang, X., Wang, A., Wang, W., Jia, J., Wei, L., Xin, Y., Qiao, Y., Huang, R., Chen, J., Han, B., Yoon, K., Hill, R.T., Zohar, Y., Chen, F., Hu, Q., Xu, J., 2014. Nannochloropsis Genomes Reveal Evolution of Microalgal Oleaginous Traits. PLOS Genet. 10. doi:10.1371/journal.pgen.1004094

Wijffels, R.H., Barbosa, M.J., 2010. An outlook on microalgal biofuels. Science (80-. ). 329, 796799. doi:10.1126/science.1189003

Xiao, Y., Zhang, J., Cui, J., Feng, Y., Cui, Q., 2013. Metabolic profiles of Nannochloropsis oceanica IMET1 under nitrogen-deficiency stress. Bioresour. Technol. 130, 731-738. doi:10.1016/j.biortech.2012.11.116

Xue, Z., Sharpe, P.L., Hong, S.-P., Yadav, N.S., Xie, D., Short, D.R., Damude, H.G., Rupert, R.A., Seip, J.E., Wang, J., Pollak, D.W., Bostick, M.W., Bosak, M.D., Macool, D.J., Hollerbach, D.H., Zhang, H., Arcilla, D.M., Bledsoe, S.A., Croker, K., McCord, E.F., Tyreus, B.D., Jackson, E.N., Zhu, Q., 2013. Production of omega-3 eicosapentaenoic acid by metabolic engineering of Yarrowia lipolytica. Nat. Biotechnol. 31, 734-740. doi:10.1038/nbt.2622

Yoon, K., Han, D., Li, Y., Sommerfeld, M., Hu, Q., 2012. Phospholipid:Diacylglycerol Acyltransferase Is a Multifunctional Enzyme Involved in Membrane Lipid Turnover and Degradation While Synthesizing Triacylglycerol in the Unicellular Green Microalga Chlamydomonas reinhardtii. Plant Cell 24, 3708-3724. doi:10.1105/tpc.112.100701

Young, E.B., Beardall, J., 2003. Photosynthetic function in Dunalliela tertiolecta (Chlorphyta) during a nitrogen starvation and recovery cycle. J. Phycol. 39, 897-905. doi:10.1046/j.1529-8817.2003.03042.x

Zemke, P.E., Sommerfeld, M.R., Hu, Q., 2013. Assessment of key biological and engineering design parameters for production of Chlorella zofingiensis (Chlorophyceae) in outdoor photobioreactors. Appl. Microbiol. Biotechnol. 97, 5645-5655. doi:10.1007/s00253013-4919-5 


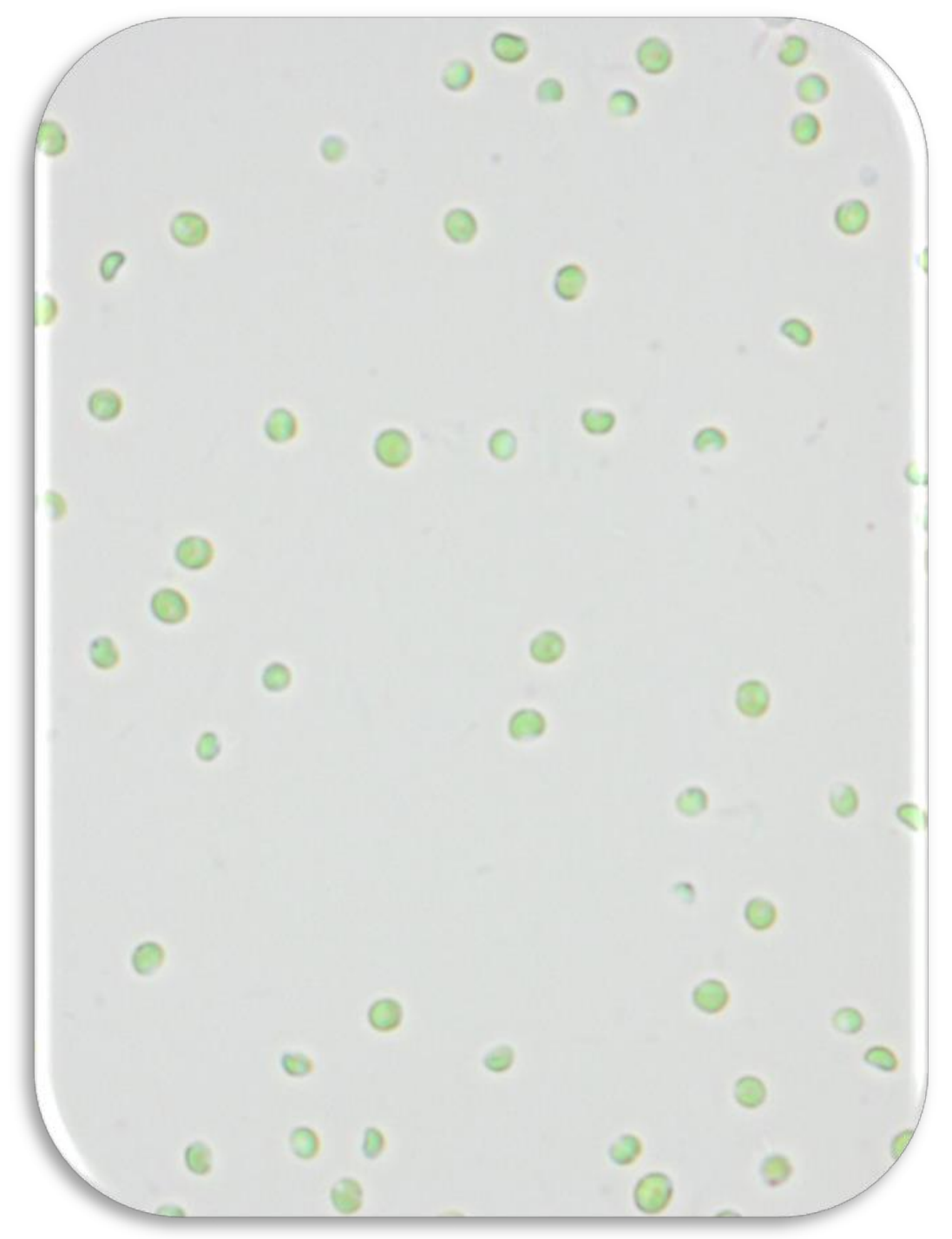

Nannochloropsis gaditana under microscope 
Summary

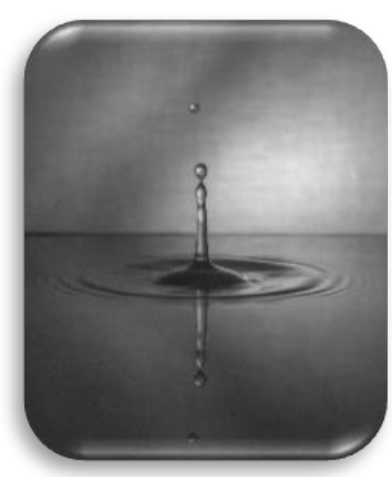




\section{SUMMARY}

Microalgae are unicellular photosynthetic microorganisms, which can convert light, carbon dioxide and nutrients into multiple products like lipids, proteins and pigments. Even though they are a sustainable source of lipids, higher lipid yields are required to turn the production into an economical feasible process. This thesis focusses on understanding the cellular mechanisms underlying lipid accumulation and developing a process with improved lipid production.

Nannochloropsis gaditana is a microalga known for its lipid accumulation. Upon nitrogen starvation it can accumulate up to $41 \%$ triacylglycerol (TAG) of its dry weight. In addition, it also produces the commercially interesting omega-3 fatty acid eicosapentaenoic acid (EPA). There are two main lipid fractions in which fatty acids accumulate; the polar lipids (PL) and triacylglycerol (TAG) lipids.

During microalgal growth EPA is mainly present in the PL. During nitrogen starvation N. gaditana accumulates large amounts of TAG in lipid bodies. When expressed per total cell dry weight, the TAG-localized EPA increases during nitrogen starvation while the PL-localized EPA decreases, suggesting that EPA is translocated from the PL into the TAG lipids during nitrogen starvation. In Chapter 2, the origin of EPA in TAG lipid bodies of $N$. gaditana was elucidated using labelled carbon $\left({ }^{13} \mathrm{C}\right)$. $N$. gaditana was first grown under optimal growth conditions with ${ }^{13} \mathrm{CO}_{2}$ as the sole carbon source followed by nitrogen starvation with ${ }^{12} \mathrm{CO}_{2}$ as the sole carbon source. By measuring both ${ }^{12} \mathrm{C}$ and ${ }^{13} \mathrm{C}$ fatty acid isotope species in time, de novo synthesized fatty acids and the already present fatty acids were distinguished. For the first time, translocation of EPA from the PL into the TAG fraction during nitrogen starvation was proven. Next to being translocated, EPA was synthesized de novo in both PL and TAG during nitrogen starvation. EPA was made by carbon reshuffling within the cell as well. EPA was the main fatty acid translocated, suggesting that the enzyme responsible for fatty acid translocation has a high specificity for EPA.

To study the pathways involved in de novo synthesis of TAG and EPA and translocation of EPA the expression levels of the involved genes were measured in Chapter 3. The same experimental conditions as Chapter 2 were used to allow comparison of gene expression levels with the results in which translocation and de novo synthesis was measured during nitrogen starvation. The expression levels of involved genes were analysed during the first hours of nitrogen starvation and over 
a longer nitrogen starvation period (14 days). Genes which might be involved in fatty acid translocation are phospholipid: diacylglycerol acyltransferase (PDAT) and lipases. One of the gene copies of PDAT and several lipases showed increased transcriptional levels, suggesting these enzymes might be responsible for translocation of EPA accumulation in TAG. The genes involved in de novo synthesis of EPA were several fatty acid elongases and desaturases. Most desaturases and elongases involved in de novo EPA synthesis were downregulated except the $\Delta 9$ desaturase, which was upregulated. This upregulation correlated with the increase in oleic acid. The genes involved in the TAG pathways showed complex regulation of expression levels. There are many hypothetical genes and therefore improvements in annotation are required to improve understanding of these pathways.

In addition to strain improvement, optimized process strategies can also lead to increased TAG productivity. Therefore, a new TAG production strategy was tested in Chapter 4. One of the most common strategies for TAG accumulation in microalgae is a two-step batch cultivation, which consists of a growth phase followed by nitrogen starvation. During nitrogen starvation the photosynthetic efficiency decreases leading to low lipid productivities. Previous studies have shown that addition of nitrogen to a nitrogen starved culture led to a higher photosynthetic efficiency compared to nitrogen starved conditions. The lipid yield did however not improve, which was hypothesized to be due to light energy used for improving the photosystems and could not be used for lipid accumulation. To improve both photosynthetic efficiency and lipid productivity, in Chapter 4 small amounts of nitrogen were added in the night during 14 days of nitrogen starvation. Nitrogen was added during the night, to allow recovery and avoid light energy being used for growth. Daily nitrogen concentrations were chosen to assure consumption of most part of the nitrogen during the night. Addition of nitrogen led to an improvement of the photosystem II maximum quantum yield and an increase in the dry weight and TAG concentration was achieved from day 7 of nitrogen starvation onwards compared to a culture without nitrogen addition. The maximal time-averaged triacylglycerol (TAG) yield on light was reached after 3 days of nitrogen starvation. Therefore, the improved photosynthetic activity achieved from day 7 onwards did not lead to an increase of the maximal time-averaged TAG yield on light. The nitrogen added was mainly used for protein production as shown by the higher protein concentration in the culture where nitrogen was added. 
Cultivation conditions, such as light intensity, used during nitrogen starvation can have a large impact on TAG accumulation. Therefore, the effect of the biomassspecific photon supply rate at the start of the nitrogen starvation phase was studied in Chapter 5. Outdoor light conditions were simulated and were kept constant for all experiments. The biomass-specific photon supply rate was varied by changing the biomass concentration at the onset of nitrogen starvation. High, intermediate and low average biomass-specific photon supply rates $\left(26,11\right.$ and $\left.6 \mu \mathrm{mol} \mathrm{g}{ }^{-1} \mathrm{~s}^{-1}\right)$ were achieved by applying equal incident light intensity to different biomass concentrations $\left(1.2,2.9\right.$ and $\left.5.4 \mathrm{~g} \mathrm{~L}^{-1}\right)$. The highest time-averaged TAG yield on light was found for the intermediate biomass-specific photon supply rate. Sub-optimal yields at high biomass-specific supply rates were attributed to photosaturation, photoinhibition and light falling through the reactor without being absorbed. At low biomass-specific photon supply rate sub-optimal yields were attributed to high volumetric maintenance requirements. The lowest biomass-specific photon supply rate resulted in highest biomass-specific optical cross section and polar lipids. The biomass-specific photon supply rate is an important parameter for optimization of TAG production by microalgae.

In Chapter 6, all results of previous chapters were compared and discussed. Since the biomass-specific photon supply rates had a large impact the TAG yield on light in Chapter 5 the different biomass-specific photon supply rates used in all chapters were compared. In addition to the biomass-specific photon supply rates also the light regimes in which light is supplied influenced the TAG yield on light. Simulated outdoor light condition resulted in a lower TAG yield on light. The localization of the EPA accumulation was measured in TAG and polar lipids. The lowest biomass-specific photon supply rate showed highest EPA content expressed per biomass, which were mainly present in polar lipids. Upon nitrogen starvation less EPA accumulated in TAG compared to the higher biomass-specific photon supply rates. Possible limitation in EPA accumulation are discussed and future research perspectives are given focussing on de novo synthesis and translocation of EPA. 


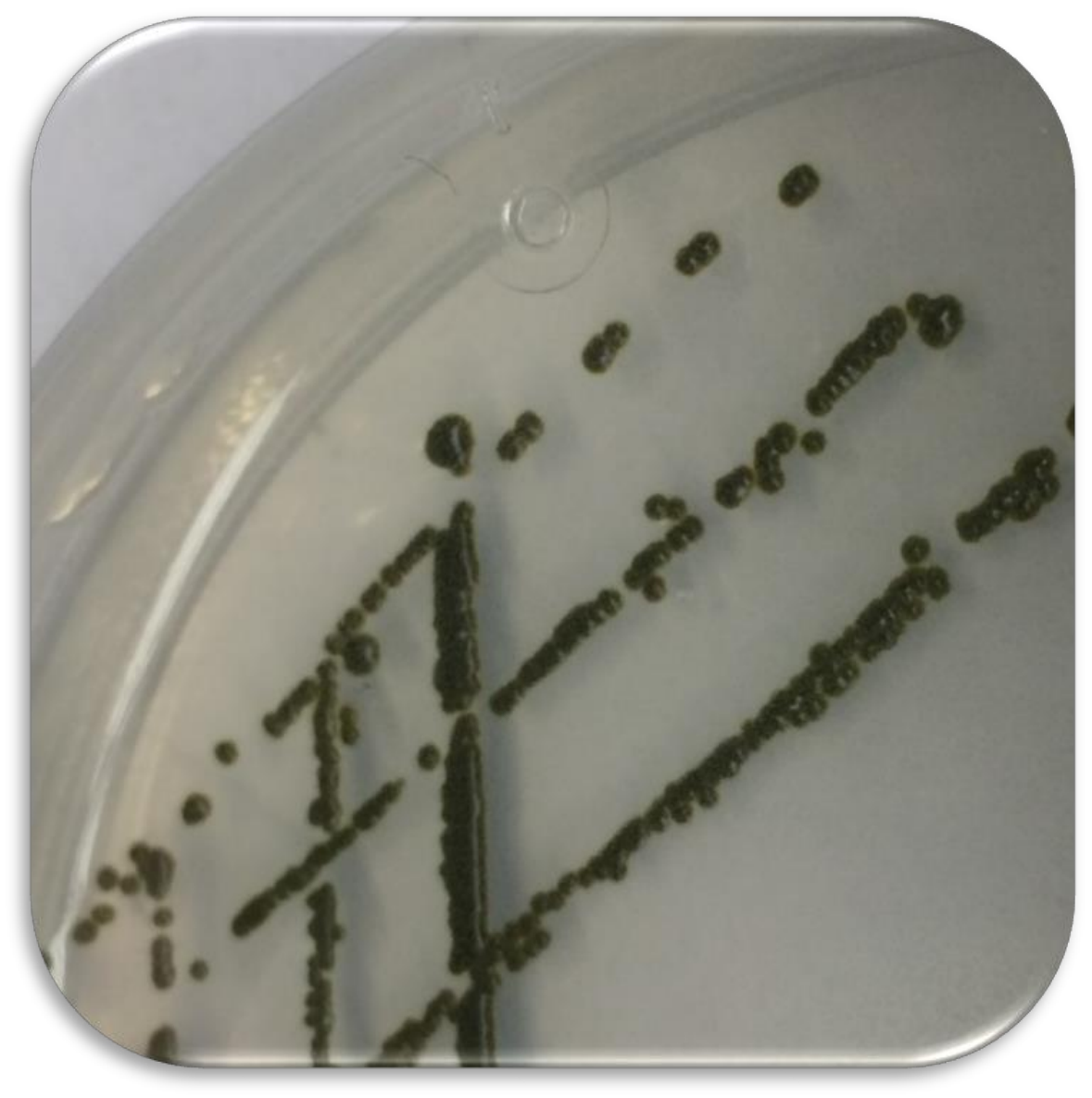

Microalgae grown on agar plates 
Samenvatting

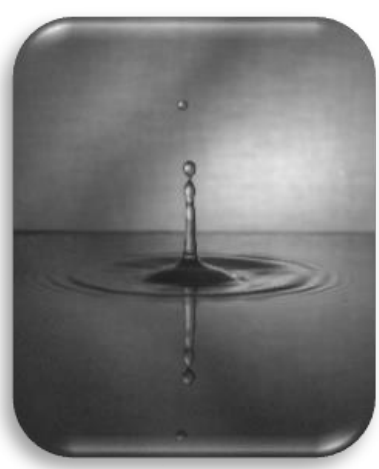




\section{SAMENVATTING}

Microalgen zijn eencellige fotosynthetische micro-organismen, die licht, koolstofdioxide en voedingsstoffen kunnen omzetten in meerdere producten zoals lipiden, eiwitten en pigmenten. Algen zijn ze een duurzame bron voor lipiden. Om de lipideproductie met microalgen economisch rendabel te maken zijn er hogere lipideopbrengsten nodig. Dit proefschrift richt zich op het inzichtelijk maken van de cellulaire mechanismen die ten grondslag liggen aan die lipideproductie en het ontwikkelen van een proces waarin de lipideproductie wordt verbeterd.

Nannochloropsis gaditana is een microalgensoort die bekend staat om de ophoping van lipiden in de vorm van triacylglycerol (TAG). Deze ophoping wordt meer door zogenoemde stresscondities, bijvoorbeeld het weghalen van de voedingstof stikstof. $\mathrm{Na}$ deze stikstofuithongering kan het opgehoopte TAG-gehalte oplopen tot wel $41 \%$ van het drooggewicht. Daarnaast produceert $N$. gaditana ook het commercieel interessante omega-3 vetzuur eicosapentaeenzuur (EPA). Vetzuren kunnen zicht in microalgaen ophopen in twee belangrijke lipidefracties, te weten de polaire lipiden (PL) en triacylglycerol (TAG).

Tijdens het groeien van de microalgen is het vetzuur EPA is voornamelijk aanwezig in de PL. Tijdens stikstofuithongering hoopt $N$. gaditana grote hoeveelheden TAG op in lipidebolletjes. De hoeveelheid EPA, uitgedrukt per droog gewicht, die tijdens stikstofuithongering aanwezig is in TAG neemt toe, terwijl de hoeveelheid EPA aanwezig in PL afneemt. Dit doet vermoeden dat EPA wordt verplaatst van de PL naar de TAG-lipiden tijdens stikstofuithongering. In Hoofdstuk 2 werd de oorsprong van EPA in lipidebolletjes van $N$. gaditana gemeten met behulp van gelabelde koolstof $\left({ }^{13} \mathrm{C}\right) . N$. gaditana werd eerst gekweekt onder optimale groeicondities met ${ }^{13} \mathrm{CO}_{2}$ als de enige koolstofbron, gevolgd door stikstofuithongering met ${ }^{12} \mathrm{CO}_{2}$ als de enige koolstofbron. Door in de tijd zowel de ${ }^{12} \mathrm{C}$ - als ${ }^{13} \mathrm{C}$-vetzuurisotopen te meten, konden compleet nieuw gesynthetiseerde vetzuren worden onderscheiden van de reeds aanwezige vetzuren. Voor het eerst was de verplaatsing van EPA uit de PL in de TAG-fractie tijdens stikstofuithongering gemeten. Naast dat het verplaatsen werd EPA tijdens stikstofuithongering compleet nieuw gesynthetiseerd in zowel PL als TAG. EPA werd daarnaast ook gemaakt door koolstofherschikking binnen de cel. EPA was het belangrijkste verplaatste vetzuur, hetgeen doet vermoeden dat het enzym dat verantwoordelijk is voor verplaatsing van vetzuren een hoge specificiteit heeft voor EPA. 
Om de metabole routes te bestuderen die betrokken zijn bij de synthese van TAG en EPA en verplaatsen van EPA werd de expressie van de betrokken genen gemeten in Hoofdstuk 3. Door dezelfde experimentele omstandigheden te gebruiken als Hoofdstuk 2, konden de gen-expressieniveaus vergeleken worden met de resultaten waar verplaatsing en nieuwe synthesis van vetzuren werd gemeten gedurende stikstofuithongering. De expressieniveaus van de betrokken genen werden geanalyseerd gedurende de eerste uren en gedurende een langere periode van stikstofuithongering (14 dagen). Genen die mogelijk betrokken zijn bij verplaatsing van vetzuren zijn fosfolipiden diacylglycerol acyltransferase (PDAT) en lipasen. Een van de genkopieën van PDAT en verschillende lipasen vertoonden verhoogde expressies, wat zou kunnen duiden op het feit dat deze enzymen verantwoordelijk zijn voor de verplaatsing van EPA naar TAG. De genen die betrokken zijn bij synthese van nieuwe EPA zijn verschillende vetzuurelongasen en desaturasen. De meeste desaturasen en elongasen, die waren betrokken bij nieuwe EPA-synthese hadden lagere expressie niveaus. Dat gold niet voor de $\Delta 9$-desaturase, die juist een verhoogde expressie had. Deze verhoogde expressie correleerde met de toename van oliezuur. De expressie van de genen die waren betrokken bij de productie van TAG vertoonden een complexe regulatie. Er zijn veel hypothetische genen aanwezig in het genoom en daarom zijn verbeteringen in gen-annotatie vereist om het begrip van deze routes te verbeteren.

Naast stamverbetering kunnen geoptimaliseerde processtrategieën ook leiden tot verhoogde TAG-productiviteit. Hoofdstuk 4 beschrijft de test en resultaten van een nieuwe TAG-productiestrategie. Een van de meest voorkomende strategieën voor TAG-ophoping in microalgen is een twee-staps-batch-cultivatiestrategie, die bestaat uit een groeifase gevolgd door stikstofuithongering. Tijdens stikstofuithongering neemt de fotosynthetische efficiëntie af, wat leidt tot een lagere lipideproductiviteit. Eerdere studies hebben aangetoond dat toevoeging van stikstof aan een stikstofuitgehongerde cultuur leidt tot een hogere fotosynthetische efficiëntie in vergelijking met de situatie waarin geen stikstof werd toegevoegd. De lipideopbrengst verbeterde echter niet. Men veronderstelde dat dit was te wijten aan het feit dat de lichtenergie die werd gebruikt vooral het fotosysteem ten goede kwam en niet de lipideproductie. Om zowel de fotosynthetische efficiëntie als de lipideproductiviteit te verbeteren, werden in Hoofdstuk 4 gedurende 14 aaneengesloten nachten van stikstofuithongering kleine hoeveelheden stikstof. De stikstof werd 's nachts toegevoegd om herstel van fotosysteem mogelijk te maken en te voorkomen dat lichtenergie gebruikt werd voor groei. De dagelijks 
toegevoegde stikstofconcentraties werden zo gekozen dat het grootste gedeelte van de stikstof tijdens de nacht werd geconsumeerd. Vergeleken met een kweek zonder stikstoftoevoeging leidde toevoeging van stikstof vanaf dag zeven van de stikstofuithongering tot een verbetering van de activiteit van het fotosysteem en ook tot een toename van het drooggewicht en TAG-concentratie. De maximale over tijd gemiddelde opbrengst aan triacylglycerol (TAG) op licht werd bereikt na drie dagen stikstofuithongering. Daarom leidde de verbeterde fotosynthetische activiteit die vanaf dag zeven werd bereikt niet tot een toename van de maximale over tijd gemiddelde TAG-opbrengst op licht. De toegevoegde stikstof werd voornamelijk gebruikt voor de productie van eiwitten, zoals blijkt uit de hogere eiwitconcentraties in de kweek waar stikstof werd toegevoegd.

Kweekomstandigheden, zoals de lichtintensiteit, die worden gebruikt tijdens de stikstofuithongering, kunnen van grote invloed zijn op de TAG-productie. Daarom werd het effect van de biomassa-specifieke lichttoevoersnelheid aan het begin van de stikstofuithongering bestudeerd in Hoofdstuk 5. De buitenlichtomstandigheden werden gesimuleerd en constant gehouden voor alle experimenten. De biomassaspecifieke lichttoevoersnelheid werd gevarieerd door de biomassaconcentratie aan het begin van stikstofuithongering te veranderen. Hoge, gemiddelde en lage gemiddelde biomassa-specifieke lichttoevoersnelheden (26, 11 en $6 \mu \mathrm{mol} \mathrm{g}^{-1} \mathrm{~s}^{-1}$ ) werden bereikt door het toepassen van gelijke invallende lichtintensiteit op verschillende biomassa concentraties $\left(1,2,2,9\right.$ en 5,4 $\left.\mathrm{g} \mathrm{L}^{-1}\right)$. De hoogste over de tijd gemiddelde TAG-opbrengst op licht werd gevonden voor de gemiddelde biomassaspecifieke lichttoevoersnelheid. Suboptimale opbrengsten bij een hoge biomassaspecifieke lichttoevoersnelheid werd toegeschreven aan verzadiging en inhibitie van het fotosysteem en doordat licht door de reactor viel zonder te worden opgenomen. $\mathrm{Bij}$ een lage biomassa-specifieke lichttoevoersnelheid werden suboptimale opbrengsten toegeschreven aan grote hoeveelheid energie die nodig is voor het onderhoud van de cellen. De laagste biomassa-specifieke licht toevoersnelheid resulteerde in de hoogste biomassa-specifieke absorptie-oppervlakte en polaire lipiden. De biomassa-specifieke lichttoevoersnelheid is dus een belangrijke parameter voor optimalisatie van TAG-productie door microalgen.

In hoofdstuk 6 werden alle resultaten van eerdere hoofdstukken vergeleken en besproken. Omdat de biomassa-specifieke lichttoevoersnelheden een grote impact bleken te hebben op de TAG-opbrengst op licht (zie Hoofdstuk 5), werden de verschillende voor biomassa-specifieke lichttoevoersnelheden in alle hoofdstukken 
vergeleken. Naast de biomassa-specifieke lichttoevoersnelheid beïnvloedt ook het lichtregimes waarin licht wordt aangevoerd de TAG-opbrengst op licht. Gesimuleerde buitenlicht omstandigheden resulteerden in een lagere TAGopbrengst op licht. De lokalisatie van de EPA werd gemeten in TAG en polaire lipiden. De laagste biomassa-specifieke licht toevoersnelheid resulteerde in het hoogste EPAgehalte uitgedrukt per biomassa, welke voornamelijk aanwezig waren in polaire lipiden. Na stikstofuithongering accumuleerde EPA minder in TAG in vergelijking met de hogere biomassa-specifieke licht toevoersnelheden. Mogelijke beperkingen in EPA-accumulatie en toekomstige onderzoeksperspectieven worden gegeven met de nadruk op de novo synthese en translocatie van EPA. 


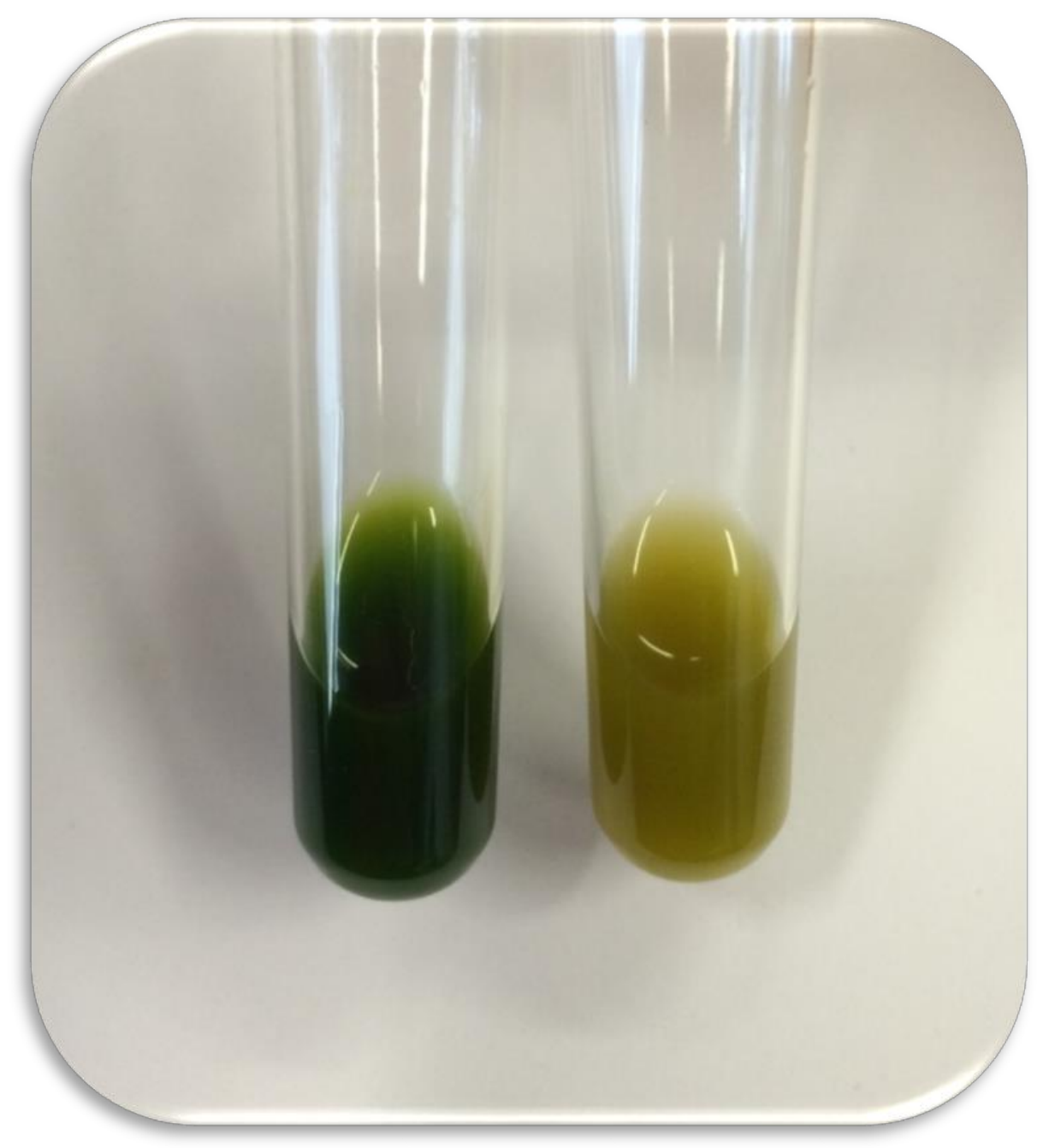

Samples tubes with healthy growing (left) and nitrogen starved (right) Nannochloropsis 
Nomenclature

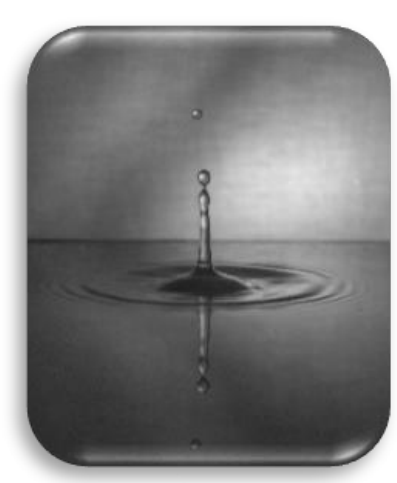




\section{NOMENCLATURE}

\begin{tabular}{|c|c|}
\hline$\alpha_{c}$ & Dry weight-specific optical cross section \\
\hline$C_{i}$ & Concentration of component $\mathrm{i}$ \\
\hline $\mathrm{CL}$ & Continuous light \\
\hline DAG & Diacylglycerol \\
\hline DGAT & Diacylglycerol acyltransferase \\
\hline DGDG & Digalactosyl diacylglycerol \\
\hline DGK & Diacylglycerol kinase \\
\hline DW & Dry weight \\
\hline EPA & Eicosapentaenoic acid \\
\hline FAS & Fatty acid synthase \\
\hline FACS & Fluorescent active cell sorting \\
\hline FFA & Free fatty acid \\
\hline $\mathrm{F}_{\mathrm{v}} / \mathrm{F}_{\mathrm{m}}$ & Photosystem II maximum quantum yield \\
\hline GPAT & Glycerol-3-phosphate acyltransferase \\
\hline LFC & $\log _{2}$ fold change \\
\hline LPAAT & Lysophosphatidic acid acyltransferase \\
\hline Lyso-PA & Lysophosphatidic acid \\
\hline MAG & Monoacylglycerol \\
\hline MGDG & Monogalactosyl diacylglycerol \\
\hline $\mathrm{Mol}_{\mathrm{ph}}$ & Mol photon \\
\hline $\mathrm{N}$ & Nitrogen \\
\hline OD & Optical density \\
\hline PCA & Principle component analysis \\
\hline PL & Polar lipid \\
\hline PS II & Photosystem II \\
\hline PUFA & Polyunsaturated fatty acid \\
\hline TAG & Triacylglycerol \\
\hline TFA & total fatty acids \\
\hline PA & Phosphatidic acid \\
\hline PAP & Phosphatidic acid phosphatase \\
\hline PC & Phosphatidylcholine \\
\hline PDAT & Phospholipid: diacylglycerol acyltransferase \\
\hline PG & Phosphatidylglycerol \\
\hline $\mathrm{PI}$ & Phosphatidylinositol \\
\hline ROS & Reactive oxygen species \\
\hline$r_{\mathrm{ph}}$ & volumetric photon supply rate $\left(\mathrm{mol}_{\mathrm{ph}} \mathrm{L}^{-1}\right.$ day $^{-1}$ ) \\
\hline $\mathrm{Y}_{\mathrm{TAG} / \mathrm{ph}}$ & Time-averaged TAG yield on light $\left(\mathrm{g}_{\mathrm{TAG}} \mathrm{mol}_{\mathrm{ph}}{ }^{-1}\right)$ \\
\hline$Y_{x, p h}$ & Biomass yield on light $\left(g_{x} \mathrm{~mol}_{\mathrm{ph}}{ }^{-1}\right)$ \\
\hline
\end{tabular}




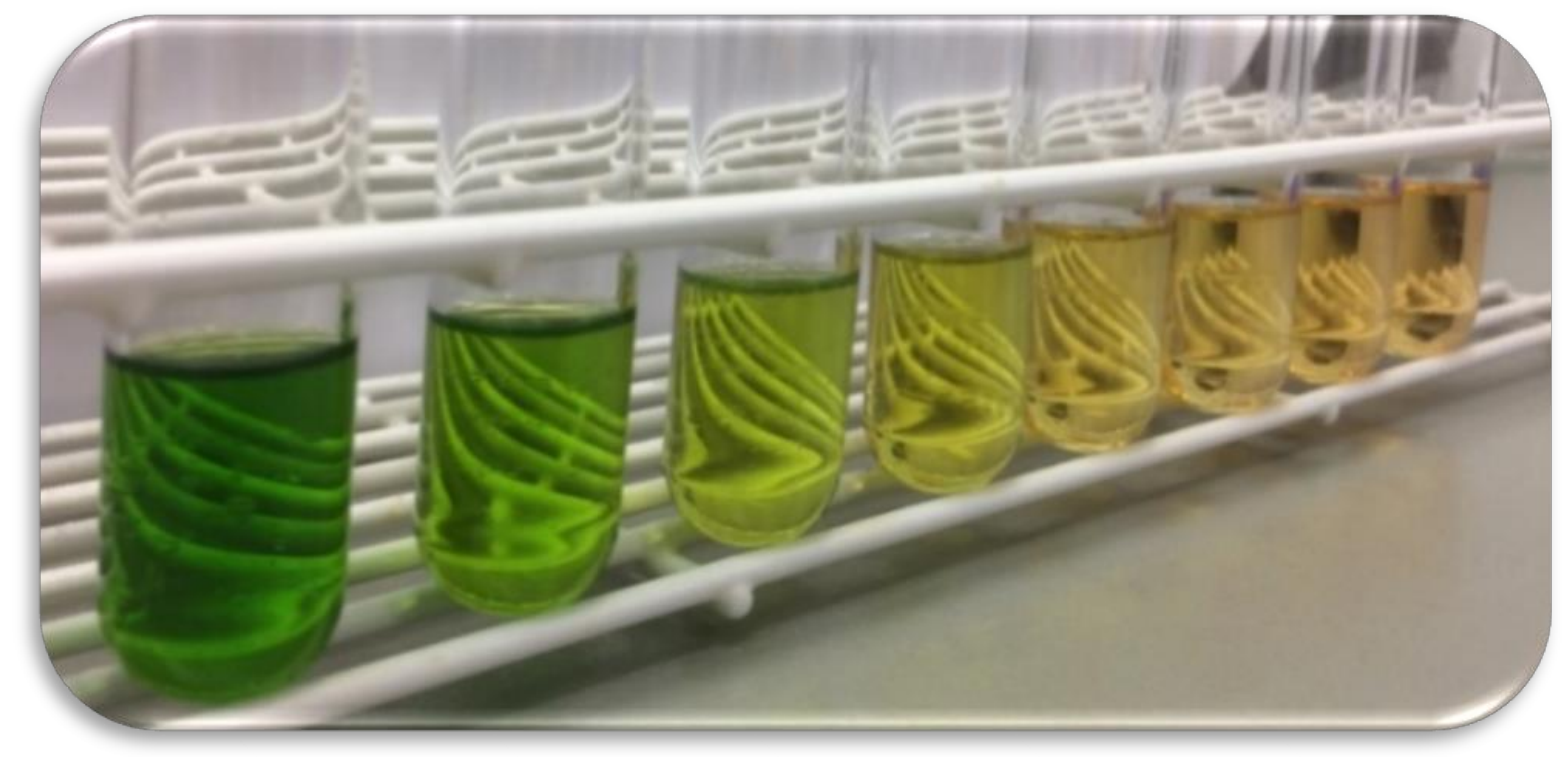

Time series of nitrogen starved Nannochloropsis gaditana during fatty acid analysis 
Acknowledgement

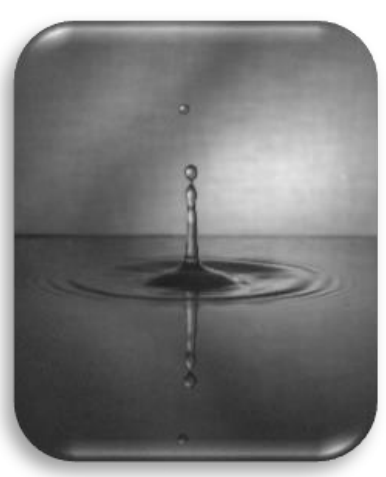




\section{ACKNOWLEDGEMENT}

A PhD is not done alone, so I would like to thank all the people who helped me during these four years. First, I would like to thank my supervisors René, Maria, Packo and Dirk. I learned a lot from you both on scientific level as well as on a personal level.

René, je bent de enige die de 4 jaar heeft volgemaakt. Bedankt voor je nuttige input tijdens onze meetings. Maria, bedankt voor je enthousiasme en alle feedback tijdens het laatste stuk van mijn PhD. Packo, bedankt voor de nuttige meetings met vele ideeën en discussies. Deze hebben veel bijgedragen aan een goede proefopzet. En bedankt voor de motivatie als niks meewerkte. Dirk, ook al was alleen aan begin betrokken bij het project, bedankt voor alle discussies.

I also had a lot of help from the students who did their thesis within the project; Timon, Jaap, Jens, Jasper and Jacco. Thank you for all your efforts and motivation. I also learned a lot from you guys. I wish you all the best for the future.

Ook willen ik mijn andere co-auteurs bedanken. Ric, bedankt voor de hulp bij het ontwikkelen en testen van de methode om ${ }^{13} \mathrm{C}$ vetzuren te analyseren. Jasper en Peter bedankt voor jullie hulp bij de transcriptome data-analyse.

De groep kan niet zonder het secretariaat. Miranda en Marina jullie staan altijd klaar om te helpen en ook voor een gezellig praatje.

Het OBP team zorgt ervoor dat alles in het lab blijft werken en voor gezelligheid op het lab. Wendy, bedankt dat je altijd klaar stond voor vragen over de analyses. Sebastiaan, je hebt er mede voor gezorgd dat we de reactor toch (zo goed als) gas dicht hebben gekregen. Fred, je was altijd bereid om te helpen als de computer van de Infors weer eens weigerde. Snezana, jij zorgt ervoor dat alles wat ik nodig aanwezig is zodat we alle experimenten kunnen doen.

Thanks to my office mates, Gerard, Xiao, Imma, Ruchir, Luci, Pieter, Fabian and Narcis for the nice times we had in the office. Gerard, je was mijn kantoorgenootje in Biotechnion en Radix bedankt voor de (niet) wetenschappelijke gesprekken.

I want to thank all the project partners involved in the MIRACLES project. Thanks for the usefull and fun meetings and discussions. Especially thanks to all WP2 and WP10 
members. Agí, we met on the first day of our PhDs. I am happy we could travel together to the MIRACLES meetings and I enjoyed our travels afterwards.

Mitsue and Ilse, I am happy that you are my paranimphs. Mitsue, I am happy we met during the PhD and became good friends. I could share my frustrations and celebrations. Ilse, bedankt voor onze vriendschap en de gezelligheid op onze reizen. Ik kijk uit naar onze volgende reis. Marta, thanks for great company, good food, and nice travels we had together.

Thanks for the lovely international dinners we had: Mitsue, Martin, Ilse, Gerard, Marta, Paulo, Rupali, Imma, Joao, Agí, Christian, Xiao and Yimin.

I would like to thank all my colleagues from BPE for the nice time. I appreciate the help in the lab but also the interesting discussion during coffeebreaks and all the nice social activities, borrels, Christmas dinners and labuitjes.

Although not directly involved in the research, I would also like to thank my family and friends who always supported me in different ways.

Lieve papa, mama en Renske jullie staan altijd voor mij klaar, of het nu is om iets te vieren of juist niet. Bedankt voor alle steun!

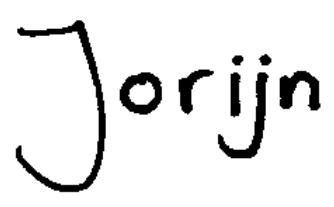




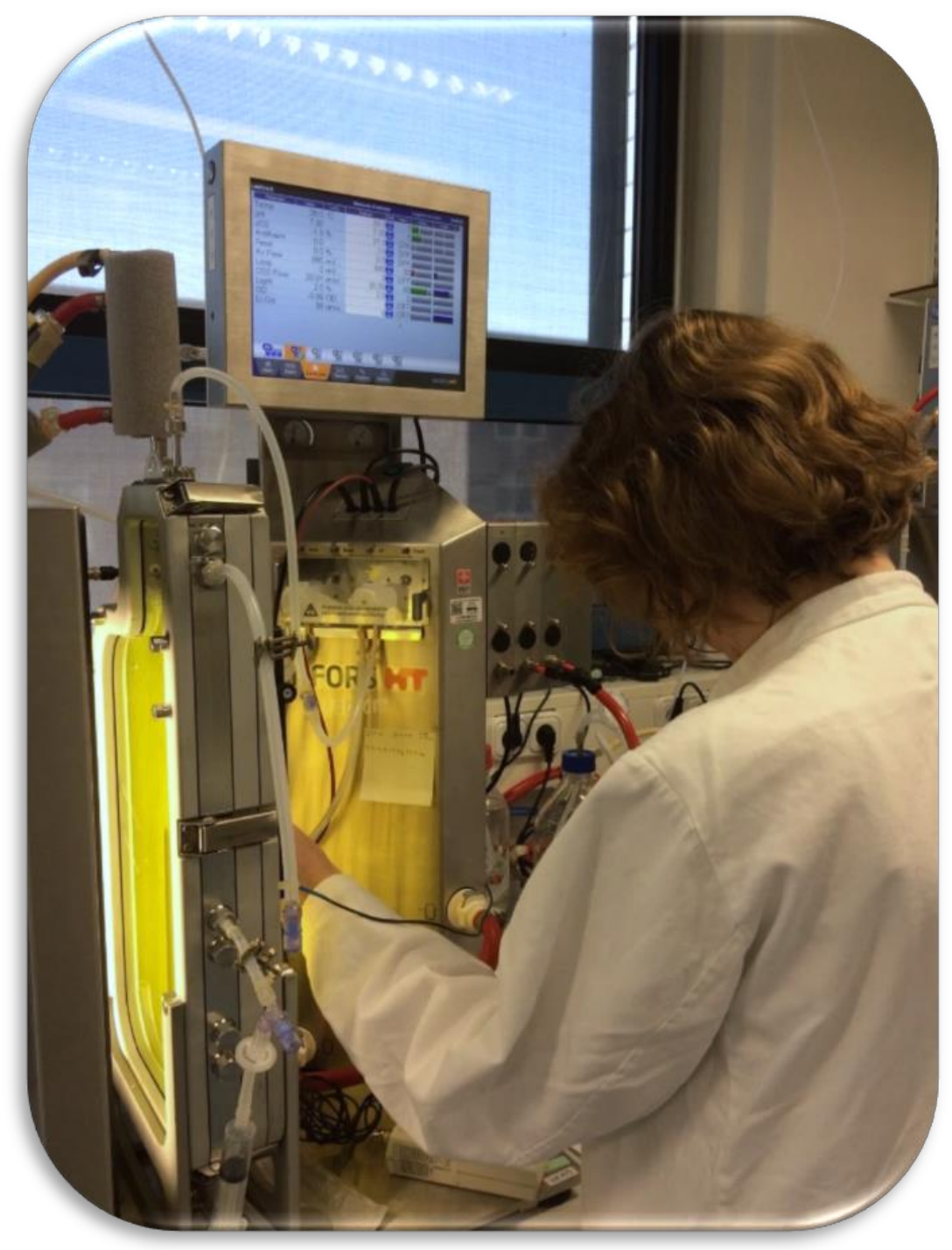

Measuring light falling through the photobioreactor 


\section{About the}

\section{author}




\section{CURRICULUM VITAE}

Jorijn Huibertine Janssen was born on June $12^{\text {th }}, 1990$ in Rotterdam, the Netherlands. She attended 'het Emmauscollege' in Rotterdam where she obtained her VWO diploma in 2008. That same year she started her Bachelor in Biotechnology at the Wageningen University and Research in Wageningen. She worked during her bachelor thesis at the laboratory of microbiology on the role of Argonaute during plasmid transformation in Thermus thermophilus. She obtained het BSc in 2011

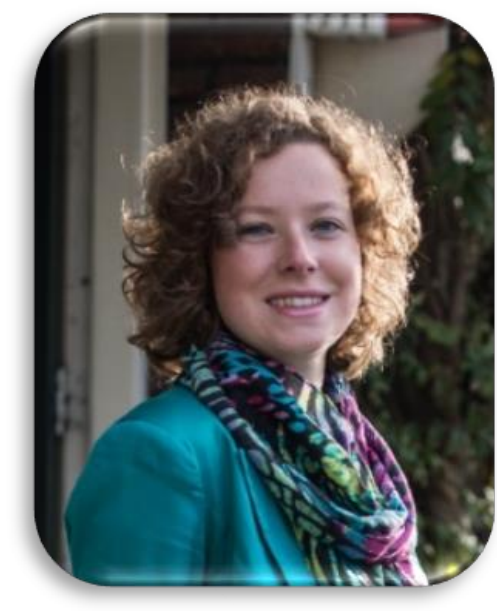
and started the Master in Biotechnology at Wageningen University and Research in Wageningen. In her MSc thesis at the Bioprocess engineering group of Wageningen University and Research, she cultivated microalgae to study the effect of light inensity on growth and pigment accumulation in Chlorella zofiengiensis. For her internship she visited the Marine microbiology group at the University of Bergen in Bergen, Norway, where she worked on the fatty acid composition of Phaeodactylum tricornutum grown at different nitrate concentration and light intensities and isolation of new microalgal strains for fatty acid production. After obtaining her MSc degree in 2013, she started her doctorate (PhD) research at the Bioprocess Engineering group at Wageningen University and Research. Her PhD project was carried out within the EU FP7 funded 'MIRACLES' program, where she studied the lipid production in the microalgae Nannochloropsis gaditana. The results of her PhD research are described in this thesis. Currently, she is working as a post-doctoral researcher at the Bioprocess engineering group at Wageningen University.

Contact information: jorijnjanssen@gmail.com 


\section{LIST OF PUBLICATIONS}

J.H. Janssen, R.H. Wijffels and M.J. Barbosa, Lipid production in Nannochloropsis gaditana, submitted for publication

J.H. Janssen, J.L.S.P. Driessen, P.P. Lamers, R.H. Wijffels and M.J. Barbosa, Effect of initial biomass-specific photon supply rate on fatty acid accumulation in nitrogen depleted Nannochloropsis gaditana under simulated outdoor light conditions, submitted for publication

J.H. Janssen, J. Kastenhofer, J.A. de Hoop, P.P. Lamers, R.H. Wijffels, M.J. Barbosa, Effect of nitrogen addition on lipid productivity of nitrogen starved Nannochloropsis gaditana, accepted for publication in Algal Research

J.H. Janssen, J. Spoelder, J.J. Koehorst, P.J. Schaap, R.H. Wijffels and M.J. Barbosa, Expression level of genes involved in TAG and polyunsaturated fatty acid synthesis of Nannochloropsis gaditana during short- and long-term nitrogen starvation, to be submitted for publication

J.H. Janssen, P.P. Lamers, R.C.H. de Vos, R.H. Wijffels, M.J. Barbosa, Translocation and de novo synthesis of eicosapentaenoic acid (EPA) during nitrogen starvation in Nannochloropsis gaditana, submitted for publication

K.J.M. Mulders, J.H. Janssen, D.E. Martens, R.H. Wijffels, P.P. Lamers, Effect of biomass concentration on secondary carotenoids and triacylglycerol (TAG) accumulation in nitrogen-depleted Chlorella zofingiensis, Algal Res. 6 (2014) 8-16. doi:10.1016/j.algal.2014.08.006.

D.C. Swarts, M.M. Jore, E.R. Westra, Y. Zhu, J.H. Janssen, A.P. Snijders, Y. Wang, D.J. Patel, J. Berenguer, S.J.J. Brouns, J. van der Oost, DNA-guided DNA interference by a prokaryotic Argonaute, Nature. 507 (2014) 258-261. doi:10.1038/nature12971. 


\section{OVERVIEW OF COMPLETED TRAINING ACTIVITIES}

\section{Discipline specific activities}

\section{Conferences}

Mini-symposium: 'Microalgae biotechnology: towards industrial strains 2013 and processes'

Mini-symposium: 'The box of Pandora: what came out? 2014

$2^{\text {nd }}$ edition Young algaeneer symposium, (Montpellier/Narbonne, France) 2014

CeBiTEc research conference: 'Prospects and challenges for the 2014 development of algal biotechnology'(Bielefeld, Germany)

Mini-symposium: 'The dynamics of oil accumulation' 2015

AlgaeBiorefineries for Europe Conference ${ }^{1}$ 2017

Algae Biomass Summit (Salt Lake City, USA) ${ }^{2}$ 2017

\section{Courses}

BSDL Advanced course Bioprocess design

VLAG Microalgae process design: from cells to photobioreactors

BSDL Advanced course Metabolomics for microbial system biology

\section{General courses}

VLAG PhD week

WGS Presenting with impact 2015

WGS Scientific writing

WGS Effective behaviour in your professional surroundings

WGS Career perspectives

\section{Optionals}

Preparation of research proposal

International PhD study trip (Portugal) ${ }^{1,2}$

BPE Symposia and brainstorm days ${ }^{2}$

MIRACLES EU consortium meetings ${ }^{2}$

${ }^{1}$ Poster; ${ }^{2}$ Oral presentation 
This study was carried out at the department of Bioprocess Enginering of Wageningen University and Research, Wageningen, the Netherlands. The research described in this thesis is performed within the EU FP7 MIRACLES project under grant agreement No. 613588.

This thesis was printed by Digiforce || ProefschriftMaken (250 copies)

Cover design by Jorijn $\mathrm{H}$. Janssen

Jorijn H. Janssen, 2018 
